\title{
Set-based Particle Swarm Optimization for Portfolio Optimization
}

by

\author{
Kyle Harper Erwin
}

Thesis presented in partial fulfilment of the requirements for the degree of Master of Computer Science in the Faculty of Science at Stellenbosch University

Supervisor: Prof. A. Engelbrecht

December 2021 


\section{Declaration}

By submitting this thesis electronically, I declare that the entirety of the work contained therein is my own, original work, that I am the sole author thereof (save to the extent explicitly otherwise stated), that reproduction and publication thereof by Stellenbosch University will not infringe any third party rights and that I have not previously in its entirety or in part submitted it for obtaining any qualification.

Date: $\quad \ldots$ December 2021

Copyright (C) 2021 Stellenbosch University All rights reserved. 


\title{
Abstract
}

\section{Set-based Particle Swarm Optimization for Portfolio Optimization}

\author{
Kyle Harper Erwin \\ Department of Computer Science, \\ University of Stellenbosch, \\ Banghoek Rd $\mathscr{G}$ Joubert Rd, Stellenbosch, 7600, South Africa.
}

Thesis: MSc (CS)

December 2021

Portfolio optimization is a complex problem, not only in the depth of the topics it covers but also in breadth. It is the process of determining which assets to include in a portfolio while simultaneously maximizing profit and minimizing risk. Portfolio optimization is rich with interesting research not only by researchers in finance, but also in computer science. The overlap of these fields has lead to an increase in the use of meta-heuristics to make intelligent investment decisions. This thesis conducts a thorough investigation into the current state of evolutionary and swarm intelligence algorithms for portfolio optimization. The investigation showed that these algorithms suffer from stability issues for larger portfolio optimization problems. A new approach using set-based particle swarm optimization (SBPSO) is proposed to reduce the dimensionality, and therefore complexity, of portfolio optimization problems. The results show that SBPSO is capable of obtaining good-quality solutions while being relatively fast. New set-based diversity measures are developed in order to better understand the exploration and exploitation behaviour of SBPSO, and set-based algorithms in general. It is shown that SBPSO fails to converge to a single solution and uses an inadequate process to determine the contribution of each asset to the portfolio. Based on these findings, improvements are made to the proposed SBPSO approach that yield significant gains in performance. The first multi-objective adaptation of SBPSO is also developed and is shown to scale to larger portfolio problems better than the multi-guided particle swarm optimization (MGPSO) algorithm, with lower levels of risk. 


\title{
Uittreksel
}

\section{Set-based Particle Swarm Optimization for Portfolio Optimization}

\author{
Kyle Harper Erwin \\ Department of Computer Science, \\ University of Stellenbosch, \\ Banghoek Rd $\mathscr{G}$ Joubert Rd, Stellenbosch, 7600, South Africa.
}

Tesis: MSc (CS)

Desember 2021

Portefeulje-optimering is ' $n$ ingewikkelde probleem, nie net in die diepte van die onderwerp wat oorgesien word nie maar ook in geheel. Dit is die proses om vas te stel watter bates om in 'n portefeulje op te neem, terwyl gelyktydig die wins te maksimeer en risiko te minimeer. Portefeulje-optimalisering is ryk aan interessante navorsing nie net deur finansiële navorsers nie, maar ook in rekenaarwetenskap. Die oorvleueling van hierdie velde het gelei tot 'n toename in die gebruik van meta-heuristieke om intelligente beleggingsbesluite te neem. Hierdie tesis sluit 'n deeglike ondersoek in na die huidige stand van evolusie en swerm intelligensie algoritmes vir portefeulje-optimalisering. Die ondersoek het getoon dat hierdie algoritmes aan stabiliteitskwessies ly vir groter portefeuljeoptimalisering probleme. 'n Nuwe benadering met behulp van versameling gebaseerde deeltjieswerm optimering (SBPSO) word voorgestel om die dimensionaliteit, en dus die kompleksiteit, van portefeulje-optimaliseringsprobleme te verminder. Die resultate toon aan dat SBPSO in staat is om oplossings van goeie gehalte te verkry, terwyl dit relatief vinnig is. Nuwe versameling gebaseerde diversiteitsmaatreëls word ontwikkel vir die verkenning en ontginning van SBPSO, en versameling gebaseerde algoritmes in die algemeen. Daar word aangetoon dat SBPSO nie daarin slaag om op 'n enkele oplossing te konvergeer nie, en gebruik 'n onvoldoende proses om die bydrae van elke bate tot die portefeulje te bepaal. Op grond van hierdie bevindings word verbeteringe aan die voorgestelde SBPSO-benadering aangebring wat beduidende winste in prestasies lewer. Die eerste meervoudige doelwit aanpassing van SBPSO word ook ontwikkel, en daar word gewys dat dit beter skaal na groter portefeuljeprobleme as die meervoudige-gids deeltjieswerm optimering (MGPSO) algoritme, met laer risiko vlakke. 


\section{Acknowledgements}

Firstly, I would like to thank Prof Andries Engelbrecht for the support and guidance he has provided. I'm eternally grateful for all the opportunities you have given me. I would also like to thank my mom, sister, and girlfriend for their love and support. Without them, none of this work would have been possible. Additional thanks to my sister and girlfriend for taking the time to proofread my work. Lastly, thank you to my friends for their patience and for taking an interest in my work. A special thanks to those who kept me company while I worked late. 


\section{Contents}

Declaration i

Abstract ii

Uittreksel iii

Acknowledgements iv

Contents

List of Figures vii

List of Tables viii

\begin{tabular}{lll}
\hline & Introduction & 1
\end{tabular}

1.1 Motivation . . . . . . . . . . . . . . . . . . . . . 2

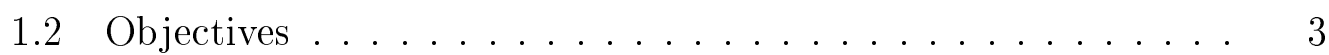

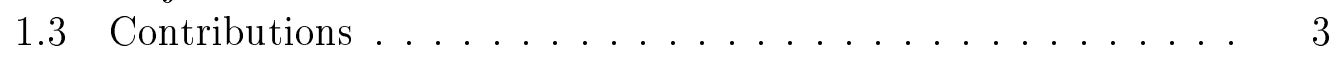

1.4 Thesis Outline ............................. 4

2 Particle Swarm Optimization $\quad 6$

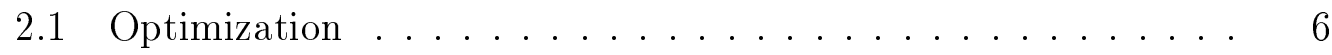

2.2 Original Particle Swarm Optimization . . . . . . . . . . . . . . 7

2.3 Multi-guide Particle Swarm Optimization . . . . . . . . . . . . . . . 10

2.4 Set-based Particle Swarm Optimization . . . . . . . . . . . . . . . . 14

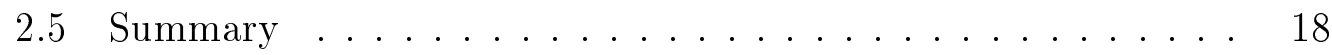

3 Portfolio Optimization 20

3.1 Introduction . . . . . . . . . . . . . . . . . . . 20

3.2 Evolutionary and Swarm Intelligence Algorithms . . . . . . . . . . 25

3.3 Unconstrained Portfolio Optimization . . . . . . . . . . . . . . . . 28

3.4 Constrained Portfolio Optimization . . . . . . . . . . . . . . . . . . . . . 30

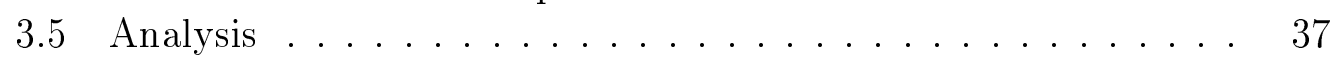

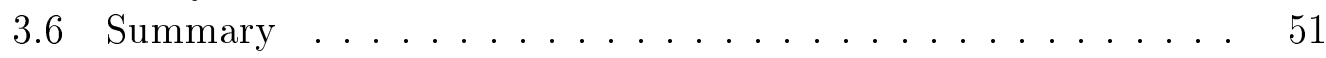


4 Set-based Particle Swarm Optimization for Portfolio Optimization 53

$4.1 \quad$ Algorithms for Portfolio Optimization . . . . . . . . . . . . . 54

$4.2 \quad$ A New Approach to Set-based Portfolio Optimization . . . . . . 58

$4.3 \quad$ Constraint Handling . . . . . . . . . . . . . . . . . . . . . . . . 61

$4.4 \quad$ Empirical Process $\ldots \ldots \ldots \ldots$. . . . . . . . . . . . . . . . . . . . . . . . . . . 61

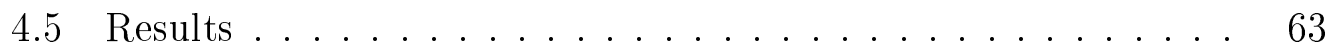

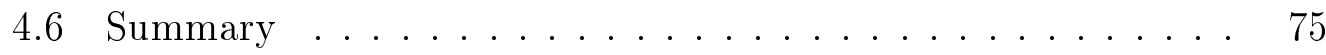

\begin{tabular}{|lll}
5 & Set-based Diversity Measures & 77
\end{tabular}

5.1 Introduction . . . . . . . . . . . . . . . . . . . 78

5.2 Set-based Distance Measures . . . . . . . . . . . . . . 78

5.3 Diversity Measures for Sets . . . . . . . . . . . . . . . . . . . . . 80

5.4 Empirical Process $\ldots \ldots \ldots \ldots \ldots$. . . . . . . . . . . . . . . . . . . . . . 83

5.5 Results . . . . . . . . . . . . . . . . . . . . . . . . . . . . 84

5.6 Summary . . . . . . . . . . . . . . . . 87

\begin{tabular}{|llll|}
\hline 6 & Set-based Particle Swarm Optimization Improvement and & \\
\hline \hline & Diversity Analysis & 89
\end{tabular}

6.1 Design Problems . . . . . . . . . . . . . . . . . . . . . . . . . . . . . . . 89

6.2 Diversity Analysis $\ldots \ldots \ldots \ldots$. . . . . . . . . . . . . . . . . . . . . . . . 90

6.3 Modifications . . . . . . . . . . . . . . . . . 91

6.4 Adaptive Coordinate Descent Weight Optimization . . . . . . 105

6.5 Modified Set-based Particle Swarm Optimization for Portfolio Optimization in Comparison with Other Algorithms . . . . . . . . . . . . . . . . . . . . . 110

6.6 Summary ............................. 125

7 Set-based Particle Swarm Optimization for Multi-objective

$\begin{array}{ll} & 127\end{array}$

7.1 Tuning-free Approach to Multi-guide Particle Swarm Optimiza-

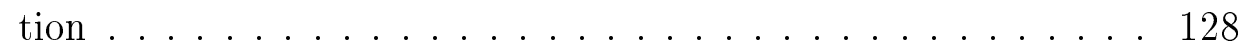

7.2 A New Approach to Multi-objective Set-based Portfolio Opti-

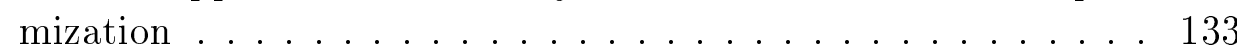

7.3 Empirical Process $\ldots \ldots \ldots \ldots \ldots . \ldots \ldots 136$

7.4 Results . . . . . . . . . . . . . . . . . . . . . . . . . . . . . . . . . . . . . . 137

7.5 Summary $\ldots \ldots \ldots \ldots \ldots \ldots \ldots \ldots$

$\begin{array}{llr}8 & \text { Conclusions } & 149\end{array}$

$8.1 \quad$ Major Findings and Conclusions. . . . . . . . . . . . . . . . . 149

8.2 Future Work . . . . . . . . . . . . . . . . . . 151

\begin{tabular}{ll}
\hline Appendices & 155
\end{tabular}

\begin{tabular}{ll}
\hline A Acronyms & 156
\end{tabular} 
\begin{tabular}{ll}
\hline B List of Symbols & 157
\end{tabular}

\begin{tabular}{ll}
\hline C Derived Publications & 161
\end{tabular}

\begin{tabular}{ll}
\hline D Control Parameter Values & 162
\end{tabular}

E Walking Fish Group Toolkit 165

E.1 Shape functions . . . . . . . . . . . . . . . . . . . . . . . . . 165

E.2 Transformation functions . . . . . . . . . . . . . . . . . . . . . . . . . . . . . . . 167

E.3 Restrictions . . . . . . . . . . . . . . . . . . . 169

E.4 Test Problems . . . . . . . . . . . . . . . . . . . 170 


\section{List of Algorithms}

$1 \quad$ Particle Swarm Optimization (Synchronous Version) . . . . . . 9

2 Multi-guide Particle Swarm Optimization . . . . . . . . . . . . . . . . . . . . 13

$3 \quad$ Archive Insert Policy $\ldots \ldots \ldots \ldots$. . . . . . . . . . . . . . . . . . .

$4 \quad$ Set-based Particle Swarm Optimization . . . . . . . . . . . . 18

$5 \quad$ Artificial Bee Colony . . . . . . . . . . . . . . . . . . 55

6 Firefly Algorithm . . . . . . . . . . . . . . . . . . . . . . 57

7 Genetic Algorithm . . . . . . . . . . . . . . . . 58

$8 \quad$ Set-based Particle Swarm Optimization for Portfolio Optimization 59

$9 \quad$ Weight Determination for Set-based Portfolio Optimization . . 59

10 Adaptive Encoding . . . . . . . . . . . . . . . . . . . . . . . 106

11 Adaptive Coordinate Descent . . . . . . . . . . . . 107

12 Multi-objective Weight Determination for Set-based Portfolio

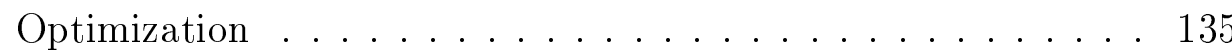

13 Multi-guide Set-based Particle Swarm Optimization . . . . . . . 136 


\section{List of Figures}

2.1 Network topologies $\ldots \ldots \ldots \ldots \ldots \ldots$

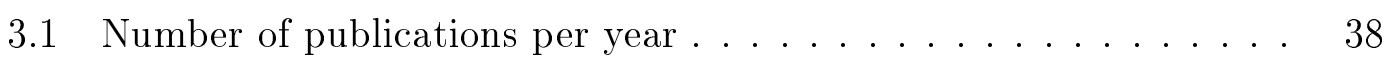

4.1 Obtained Pareto-optimal fronts for Hang Seng . . . . . . . . . . 65

4.2 Obtained Pareto-optimal fronts for DAX 100 . . . . . . . . . 67

4.3 Obtained Pareto-optimal fronts for FTSE 100. . . . . . . . . . 70

4.4 Obtained Pareto-optimal fronts for the S\&P 100 benchmark . . . . 72

4.5 Obtained Pareto-optimal fronts for the Nikkei 225 benchmark . . . 75

5.1 Jaccard Diversity (blue) and Hamming Diversity (orange) for Ex-

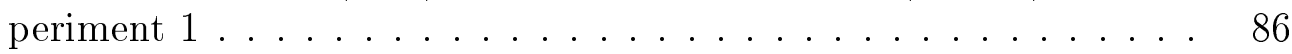

$5.2 \quad$ Jaccard Diversity (blue) and Hamming Diversity (orange) for Ex-

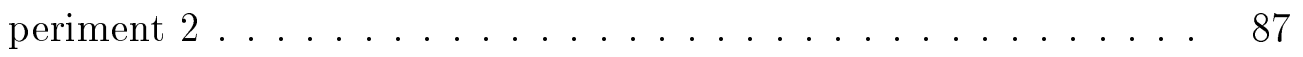

6.1 Average Jaccard diversity over time for SBPSO . . . . . . . . . . . 91

6.2 Average Jaccard Diversity over Time for $\mathrm{SBPSO}_{\mathrm{m} 1} \ldots \ldots \ldots . . . \quad \ldots 2$

6.3 Average Jaccard Diversity over Time for $\mathrm{SBPSO}_{\mathrm{m} 2} \ldots \ldots \ldots . . \quad \ldots 95$

$\begin{array}{lll}6.4 & \text { Average Jaccard Diversity over Time for } \mathrm{SBPSO}_{\mathrm{m} 3} & \ldots \ldots \ldots\end{array}$

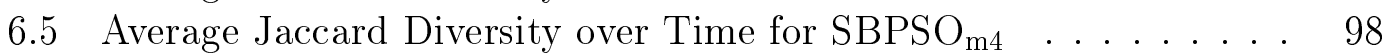

6.6 Average Jaccard Diversity over Time for $\mathrm{SBPSO}_{\mathrm{m} 5, \tau=3} \ldots \ldots . .101$

6.7 Average Jaccard Diversity over Time for $\mathrm{SBPSO}_{\mathrm{m} 5, \tau=5} \ldots \ldots . .101$

6.8 Average Jaccard Diversity over Time for $\mathrm{SBPSO}_{\mathrm{m} 5, \tau=10} \ldots \ldots . .102$

6.9 Average Jaccard Diversity over Time for $\mathrm{SBPSO}_{\mathrm{m} 6} \ldots \ldots \ldots$

6.10 Average Jaccard Diversity over Time for $\mathrm{SBPSO}_{\mathrm{ACD}} \ldots \ldots$. . . . . 109

6.11 Obtained Pareto-optimal fronts for Hang Seng . . . . . . . . . 113

6.12 Obtained Pareto-optimal fronts for DAX 100 . . . . . . . . 116

6.13 Obtained Pareto-optimal fronts for FTSE 100. . . . . . . . . . . 119

6.14 Obtained Pareto-optimal fronts for S\&P $100 \ldots \ldots$. . . . . . . 122

6.15 Obtained Pareto-optimal fronts for Nikkei 225 . . . . . . . . 125

7.1 MGSBPSO Structure . . . . . . . . . . . . . . . . . . . . 134

7.2 Obtained Pareto-optimal fronts for Hang Seng . . . . . . . . . 139

7.3 Obtained Pareto-optimal fronts for DAX 100 . . . . . . . . . . 141

7.4 Obtained Pareto-optimal fronts for FTSE 100. . . . . . . . . . . 143

7.5 Obtained Pareto-optimal fronts for S\&P 100 . . . . . . . . . 145 
7.6 Obtained Pareto-optimal fronts for Nikkei 225 . . . . . . . . . . 147 


\section{List of Tables}

3.1 Representation of evolutionary and swarm intelligence paradigms

\begin{tabular}{|ll}
\hline \hline for unconstrained and constrained portfolio optimization . . . . . . 39 \\
\hline
\end{tabular}

3.2 Evolutionary algorithms for portfolio optimization . . . . . . . . . . 41

3.4 Commonly considered constraints for portfolio optimization . . . . 44

3.5 The number of constraints considered for portfolio optimization per number of objectives . . . . . . . . . . . . 45

3.6 Summary of the OR Library data sets for portfolio optimization . 48

3.3 $\quad$ Swarm intelligence algorithms for portfolio optimization . . . . . 52

4.1 Parameter ranges used in the tuning process . . . . . . . . . . . . . . 62

4.2 Hang Seng results for each performance measure . . . . . . . . . . . . . . 64

4.3 Hang Seng rankings for each performance measure . . . . . . . . . . . . . . 64

4.4 DAX 100 results for each performance measure . . . . . . . . . . . . . 66

4.5 DAX 100 rankings for each performance measure. . . . . . . . . . . . . 66

4.6 FTSE 100 results for each performance measure . . . . . . . . . . . 68

4.7 FTSE 100 rankings for each performance measure . . . . . . . . . . . . . . 69

4.8 S\&P 100 results for each performance measure . . . . . . . . . . . . . 71

4.9 S\&P 100 rankings for each performance measure . . . . . . . . . . . . . 71

4.10 Nikkei 225 results for each performance measure . . . . . . . . . . . . 73

4.11 Nikkei 225 rankings for each performance measure . . . . . . . . . . 74

6.1 SBPSO versus SBPSO $_{\mathrm{m} 1}$ : Number of zero-weight assets for each

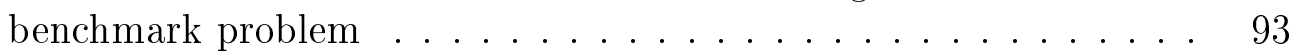

$6.2 \quad$ SBPSO versus SBPSO $_{\mathrm{m} 1}$ : Mean and standard deviations for each performance measure ....................... 94

$6.3 \quad$ SBPSO $_{\mathrm{m} 1}$ versus $\mathrm{SBPSO}_{\mathrm{m} 2}$ : Mean and standard deviations for each performance measure ..................... 95

$6.4 \quad \mathrm{SBPSO}_{\mathrm{m} 2}$ versus $\mathrm{SBPSO}_{\mathrm{m} 3}$ : Mean and standard deviations for each performance measure .................. 96

$6.5 \quad$ SBPSO $_{\mathrm{m} 3}$ versus $\mathrm{SBPSO}_{\mathrm{m} 4}:$ Mean and standard deviations for each performance measure ................... 99

$6.6 \quad$ SBPSO $_{\mathrm{m} 4}$ versus $\mathrm{SBPSO}_{\mathrm{m} 5}:$ Mean and standard deviations for each performance measure . . . . . . . . . . . . 100

$6.7 \quad$ SBPSO $_{\mathrm{m} 4}$ versus $\mathrm{SBPSO}_{\mathrm{m} 6}:$ Mean and standard deviations for each performance measure .................. 105 
6.8 SBPSO $_{\mathrm{m} 6}$ versus $\mathrm{SBPSO}_{\mathrm{ACD}}$ : Mean and standard deviations for each performance measure . . . . . . . . . . . . . . 109

6.9 Hang Seng results for each performance measure . . . . . . . . . . . 111

6.10 Hang Seng rankings for each performance measure . . . . . . . . . 112

6.11 DAX 100 results for each performance measure . . . . . . . . . . . . 114

6.12 DAX 100 rankings for each performance measure. . . . . . . . . . . 115

6.13 FTSE 100 results for each performance measure . . . . . . . . . . . 117

6.14 FTSE 100 rankings for each performance measure . . . . . . . . . 118

6.15 S\&P 100 results for each performance measure . . . . . . . . . . . 120

6.16 S\&P 100 rankings for each performance measure . . . . . . . . . . . 121

6.17 Nikkei 225 results for each performance measure . . . . . . . . . . . 123

6.18 Nikkei 225 rankings for each performance measure . . . . . . . . . . 124

7.1 MGPSO control parameters used for each WFG test problem . . . 130

7.2 MGPSO versus MGPSO $_{\mathrm{s}}$ : Mean results for 2-objective WFG . . . 131

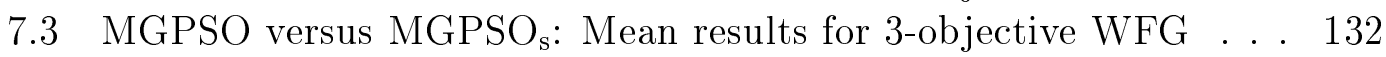

7.4 2-objective WFG rankings for each performance measure . . . . . . 132

7.5 3-objective WFG rankings for each performance measure . . . . . . 133

7.6 Hang Seng results for each performance measure . . . . . . . . . . . 138

7.7 Hang Seng rankings for each performance measure . . . . . . . . . . 138

7.8 DAX 100 results for each performance measure . . . . . . . . . . . . 140

7.9 DAX 100 rankings for each performance measure. . . . . . . . . . . 140

7.10 FTSE 100 results for each performance measure . . . . . . . . . . . 142

7.11 FTSE 100 rankings for each performance measure . . . . . . . . . . 142

7.12 S\&P 100 results for each performance measure . . . . . . . . . . . . 144

7.13 S\&P 100 rankings for each performance measure . . . . . . . . . . . 144

7.14 Nikkei 225 results for each performance measure . . . . . . . . . . . . 146

7.15 Nikkei 225 rankings for each performance measure . . . . . . . . . . 146

D.1 Tuned control parameters for the Hang Seng benchmark problem for each algorithm . . . . . . . . . . . . . . . 162

D.2 Tuned control parameters for the DAX 100 benchmark problem for

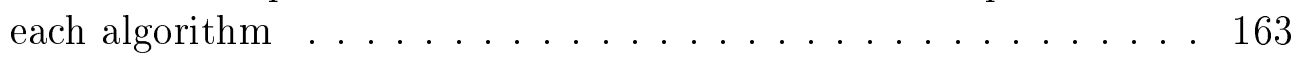

D.3 Tuned control parameters for the FTSE 100 benchmark problem for each algorithm . . . . . . . . . . . . . . 163

D.4 Tuned control parameters for the S\&P 100 benchmark problem for

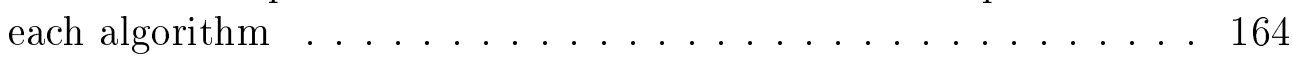

D.5 Tuned control parameters for the Nikkei 225 benchmark problem for each algorithm . . . . . . . . . . . . . . . 164

E.1 Properties of the WFG problems . . . . . . . . . . . . . 173 


\section{Chapter 1}

\section{Introduction}

An investment in knowledge always pays the best interest

-Benjamin Franklin,

The Way to Wealth: Ben Franklin on Money and Success

The practice of investing has allowed many ordinary people to build longterm wealth. These investments are typically made with an objective in mind, such as savings for retirement, a rainy day, or to fund expensive purchases. Not only can investing be good for the individual, but also for society as it can lead to job creation, increased spending and overall economic growth. Put simply, investing is the commitment to increase prosperity over time through the transfer of current resources. There are many advantages to investing and because of these advantages, people want to make the best investment decisions as possible.

Investors purchase financial resources, referred to as assets, such as shares in a company or property, speculating that over time the monetary value of the asset will increase and can then be sold for a profit. However, it is not a guarantee that an asset's value will rise, as there is always some level of risk associated with the asset. There is no such thing as a free lunch. To illustrate the investment process, consider the game of Monopoly. In the game, players purchase property. When a player lands on another player's property, that player must then pay the owner a fee. The more expensive the property purchased, the higher the fees. Players must therefore strategize which properties to buy. In some cases, a player might take a risk and purchase an expensive property, but unfortunately, it is hardly ever visited by other players. In this scenario, the risk does not pay off and possibly jeopardises the player's position in the game. Players must determine what the best possible way to spend their money would be, in order to maximize their profits without going bankrupt.

The real world is much more complex with many moving parts, but it is similar to Monopoly in that investing can be a risky venture. However, it is possible that an asset's value increases significantly, making it a worthwhile 
investment. Identifying which asset, or collection of assets known as a portfolio, would yield an optimal balance between risk and reward is not an easy task. If it was, people would need to be akin to fortune-tellers.

Portfolio optimization is the process of determining well-performing assets, and their corresponding contribution to a portfolio, that will maximize profits while mitigating risk. Portfolio optimization is a complex, multifaceted problem where computational intelligence techniques have found great success over traditional methods. However, the performance of these aforementioned computational intelligence techniques has been known to deteriorate for larger portfolio optimization problems 183]. This thesis redefines portfolio optimization as a set-based selection problem and proposes set-based algorithms for said problem.

The motivation for this study is discussed in Section 1.1. Section 1.2 lists the objectives of this study. The contributions made throughout this study are listed in Section 1.3. Finally, an outline of the remainder of this thesis is given in Section 1.4 .

\subsection{Motivation}

There is no single solution to the portfolio optimization problem, but rather a set of optimal trade-offs between return and risk. Knowledge of, or an approximation of these trade-offs is valuable information that could aid in the decision-making process behind investments. Portfolio optimization has been studied extensively by researchers in computer science and finance, with new and novel work frequently published [86]. Traditional approaches to portfolio optimization, for example quadratic programming, can correctly identify solutions for simple portfolio models. However, investors often face constraints and multiple, sometimes conflicting objectives that render traditional methods computationally ineffective. As a result, researchers have turned to metaheuristic approaches to approximate optimal solutions efficiently.

Meta-heuristic algorithms have been shown to efficiently identify optimal solutions for a variety of complex and unique portfolio models [86], [133. Yet, these approaches are also not without their flaws. When the number of assets under consideration increases, that is, the dimensionality of the problem increases, performance diminishes [183]. This deterioration in performance is commonly referred to as the curse of dimensionality.

This study presents a new approach to portfolio optimization, set-based particle swarm optimization (SBPSO), to address the stability issues of previous algorithms. SBPSO performs a bi-stage search process where assets are first selected to reduce the dimensionality of the problem and then optimal weighting for each asset in the portfolio is determined. It is believed that this approach will result in more finely-tuned portfolios that are more profitable and risk-averse than those obtained by other algorithms. 


\subsection{Objectives}

The objective of this study is to provide investors and researchers with new algorithms that make use of SBPSO for single and multi-objective portfolio optimization while overcoming the stability issues of previous approaches. Note that this thesis does not make any grandiose claims about the proposed algorithms being the be-all and end-all of investment approaches. Rather the proposed algorithms should be seen as another tool in an investor's arsenal to aid him in his investment decisions. After all, in a 2009 letter to shareholders, the legendary investor Warren Buffett said "beware of geeks bearing formulas".

These algorithms need to be able to identify a diverse set of optimal and practical solutions efficiently without succumbing to the curse of dimensionality. In working towards this goal, the following sub-objectives have been identified:

- to provide an overview of existing meta-heuristic techniques, namely swarm intelligence and evolutionary algorithms, that have been used to solve the portfolio-optimization problems.

- to investigate qualitative measures for calculating the diversity of a collection of sets.

- to propose an SBPSO for single-objective portfolio optimization problem that addresses scalability issues of previous approaches.

- to develop a multi-objective adaptation of SBPSO for multi-objective portfolio optimization problems.

- to investigate the performance of the above-mentioned algorithms for solving portfolio optimization problems.

- to compare the performance of the above-mentioned algorithms with state-of-the-art algorithms.

- to identify key areas for future work based on the findings of this study.

\subsection{Contributions}

The main contributions of this study are:

- A thorough investigation into current state of portfolio optimization with respect to evolutionary and swarm intelligence algorithms.

- A proposed method of solving the portfolio optimization problem using SBPSO. 
- The finding that SBPSO addresses the stability issues of previous approaches and can find good-quality solutions quickly.

- Methods for calculating set-based diversity using the Jaccard distance and the Hamming distance measures.

- Several improvements to the proposed SBPSO approach that increase performance.

- A variant of the proposed SBPSO approach that uses adaptive coordinate descent (ACD) that is shown to be good at identifying high quality portfolios but at the cost of a longer search time.

- The finding of an alternative approach to parameter tuning of the multiguide particle swarm optimization (MGPSO) algorithm that randomly samples control parameter values from the theoretically derived stability region.

- A multi-objective adaptation of SBPSO for multi-objective portfolio optimization that incorporates elements from MGPSO, namely multi-guide set-based particle swarm optimization (MGSBPSO).

- The finding that the MGSBPSO approach can scale to larger portfolio problems better than MGPSO.

\subsection{Thesis Outline}

- Chapter 2 provides the necessary background on optimization, and particle swarm optimization (PSO) and its concepts. MGPSO and SBPSO are also discussed in detail.

- Chapter 3 presents the necessary background on portfolio optimization and a thorough review and analysis of existing evolutionary and intelligence algorithms for portfolio optimization.

- Chapter 4 proposes SBPSO for portfolio optimization and investigates its performance.

- Chapter 5 investigates two approaches to measuring the diversity of sets using the Jaccard distance and the Hamming distance measures.

- Chapter 6 conducts a diversity and performance analysis of SBPSO, from which several improvements are made.

- Chapter 7 proposes MGSBPSO for multi-objective portfolio optimization and investigates its performance. An alternative strategy to parameter tuning for MGPSO is also investigated. 
- Chapter 8 concludes the thesis and provides a summary of all the findings of the presented work. Suggestions for future research are also given.

- Appendix A provides a list of the important acronyms used or newly defined in the course of this work, as well as their associated definition.

- Appendix B provides a list of the important symbols used or newly defined in the course of this work, as well as their associated definition.

- Appendix C provides a list of the derived publications form this work.

- Appendix D provides the tuned control parameters for each algorithm for portfolio optimization.

- Appendix E details the Walking Fish Group (WFG) toolkit used to benchmark the performance of MGPSO and the proposed approach to randomly sample control parameter values from the theoretically derived stability region. 


\section{Chapter 2}

\section{Particle Swarm Optimization}

In 1995, Eberhart and Kennedy presented PSO, a stochastic population-based search algorithm inspired by the social behaviour of birds within a flock that converge to a single point [49]. PSO has since become an extremely popular choice for optimization problems, with many subsequent adaptations and improvements proposed by other researchers. The objective of this chapter is to provide the reader with the necessary background on PSO and its concepts. Two other PSO algorithms are also discussed, each developed for a specific type of optimization problem. Furthermore, all of the PSO algorithms presented in this chapter are used in later chapters.

An introduction to optimization problems is given in Section 2.1. Section 2.2 introduces the original PSO algorithm. A multi-objective adaptation of PSO, MGPSO, is presented in Section 2.3. SBPSO is discussed in Section 2.4 . Section 2.5 summarises the chapter.

\subsection{Optimization}

The goal of an optimization algorithm is to find a solution to an optimization problem, such that a given quantity is optimized, possibly subject to a set of constraints [52]. Portfolio optimization is such a problem. In order to better understand portfolio optimization, single and multi-objective optimization are briefly discussed in Sections 2.1.1 and 2.1.2, respectively.

\subsubsection{Single-objective Optimization}

Formally, a single-objective optimization problem, $f$, assuming minimization, is defined as

$$
\begin{aligned}
& \operatorname{minimize} f(\boldsymbol{x}), \boldsymbol{x}=\left(x_{1}, x_{2}, \ldots, x_{n}\right) \\
& \boldsymbol{x} \in \Omega
\end{aligned}
$$


where $\boldsymbol{x}$ is a $n$-dimensional decision vector within the search space, $\Omega$ [35], [52]. Each component of the decision vector corresponds to a decision variable in $f$. Solutions to $f$ are constrained to the bounds of $\Omega$.

\subsubsection{Multi-objective Optimization}

A multi-objective optimization problem (MOOP) is the simultaneous optimization of two or three conflicting objectives [35], [52], [118]. Problems that consist of four or more objectives are commonly referred to as manyobjective optimization problems $[82$. Assuming minimization, multi-objective and many-objective optimization problems are defined as

$$
\begin{gathered}
\text { minimize } \\
\boldsymbol{f}(\boldsymbol{x} \in \Omega
\end{gathered}
$$

where $m$ is the number of objectives.

The solution to a MOOP is not a single solution, but rather a set of optimal trade-offs, referred to as the Pareto-optimal solutions (POS) [35], |52|. The POS contains the vectors in the decision space that best balance the conflicting objectives. The objective function maps these decision vectors to the objective space, which forms the Pareto-optimal front (POF). The POF contains nondominated solutions, which are objective vectors that are not dominated by any other feasible solution found. Formally, a decision vector $\boldsymbol{x}_{1}$ dominates a decision vector $\boldsymbol{x}_{2}$, denoted by $\boldsymbol{x}_{1}<\boldsymbol{x}_{2}$, if and only if $f_{k}\left(\boldsymbol{x}_{1}\right) \leq f_{k}\left(\boldsymbol{x}_{2}\right) \forall k \in\{1, \cdots, m\}$ and $\exists k \in\{1, \cdots, m\}$ such that $f_{k}\left(\boldsymbol{x}_{1}\right)<f_{k}\left(\boldsymbol{x}_{2}\right)$, assuming a minimization problem. The true POF is the set of all feasible non-dominated solutions that exist within the objective space. The goal of a multi-objective algorithm is to obtain a diverse spread of non-dominated solutions that are as close to the true POF as possible [52].

\subsection{Original Particle Swarm Optimization}

PSO is a single-objective optimization algorithm that simulates the flocking behaviour of birds [49], [142]. PSO uses a population, referred to as a swarm, which is a collection of randomly initialized particles within the bounds of the optimization problem under consideration. Each particle's position represents a candidate solution to the optimization problem. The particles cooperate to solve the optimization problem by exchanging locally available information about their positions through network topologies. Particles move through the search space by means of velocity updates that guide the particles to more desirable solutions. The velocity of each particle, which is initially zero, is influenced by social and cognitive guides, as well as the previous velocity of the particle. The cognitive guide is a particle's personal best solution found 
throughout the duration of the search. The social guide is the best-known solution within a network, also referred to as a neighbourhood, of particles.

Section 2.2.1 describes the PSO search process. Inertia, used to control the velocity of particles, is discussed in Section 2.2.2. Section 2.2.3 presents two commonly used network topologies in PSO. The stability conditions on PSO control parameters are discussed in Section 2.2.4.

\subsubsection{Search Process}

Particles iteratively move through the search space by updating their position with their velocity at each time step. Velocity determines the step size and search direction of the particle. The original PSO proposed by Eberhart and Kennedy updates a particle's velocity using 49

$$
\boldsymbol{v}_{i}(t+1)=\boldsymbol{v}_{i}(t)+c_{1} \boldsymbol{r}_{1}(t)\left(\boldsymbol{y}_{i}(t)-\boldsymbol{x}_{i}(t)\right)+c_{2} \boldsymbol{r}_{2}(t)\left(\hat{\boldsymbol{y}}_{i}(t)-\boldsymbol{x}_{i}(t)\right)
$$

where $\boldsymbol{v}_{i}$ is the velocity of particle $i ; \boldsymbol{x}_{i}$ is the position of particle $i ; \boldsymbol{r}_{1}$ and $\boldsymbol{r}_{2}$ are vectors of random values sampled from a uniform distribution in the range $[0,1] ; c_{1}$ and $c_{2}$ are positive constants referred to as the cognitive and social acceleration coefficients, respectively; $\boldsymbol{y}_{i}$ is the personal best position of particle $i$ and $\hat{\boldsymbol{y}}_{i}$ is the best position within the particle's neighbourhood. Constants $c_{1}$ and $c_{2}$ are responsible for controlling the amount of influence that the cognitive and social guides have. A particle's position is updated using

$$
\boldsymbol{x}_{i}(t+1)=\boldsymbol{x}_{i}(t)+\boldsymbol{v}_{i}(t+1)
$$

Algorithm 1 contains pseudo code for PSO.

\subsubsection{Inertia}

The search behaviour of PSO should ideally balance the time spent between exploring the search space and exploiting promising areas. In other words, balance the time spent between global exploration and local exploration of the search space. Shi and Eberhart introduced an inertia weight, $w$, to the velocity update function to regulate the trade-off between exploitation and exploration [153]. The inertia-weighted velocity function is defined as

$$
\boldsymbol{v}_{i}(t+1)=w \boldsymbol{v}_{i}(t)+c_{1} \boldsymbol{r}_{1}(t)\left(\boldsymbol{y}_{i}(t)-\boldsymbol{x}_{i}(t)\right)+c_{2} \boldsymbol{r}_{2}(t)\left(\hat{\boldsymbol{y}}_{i}(t)-\boldsymbol{x}_{i}(t)\right)
$$

An inertia weight less than 1 reduces the influence of the previous velocity component, and depending on the values of $c_{1}$ and $c_{2}$, the velocity of each particle will eventually reach zero $[32$. Conversely, an inertia weight greater than 1 increases the influence of the previous velocity component and results in an explosion in a particle's velocity, leading to divergent particle behaviour. The inertia PSO is similar to Algorithm 1, but with velocities updated using equation (2.5). 


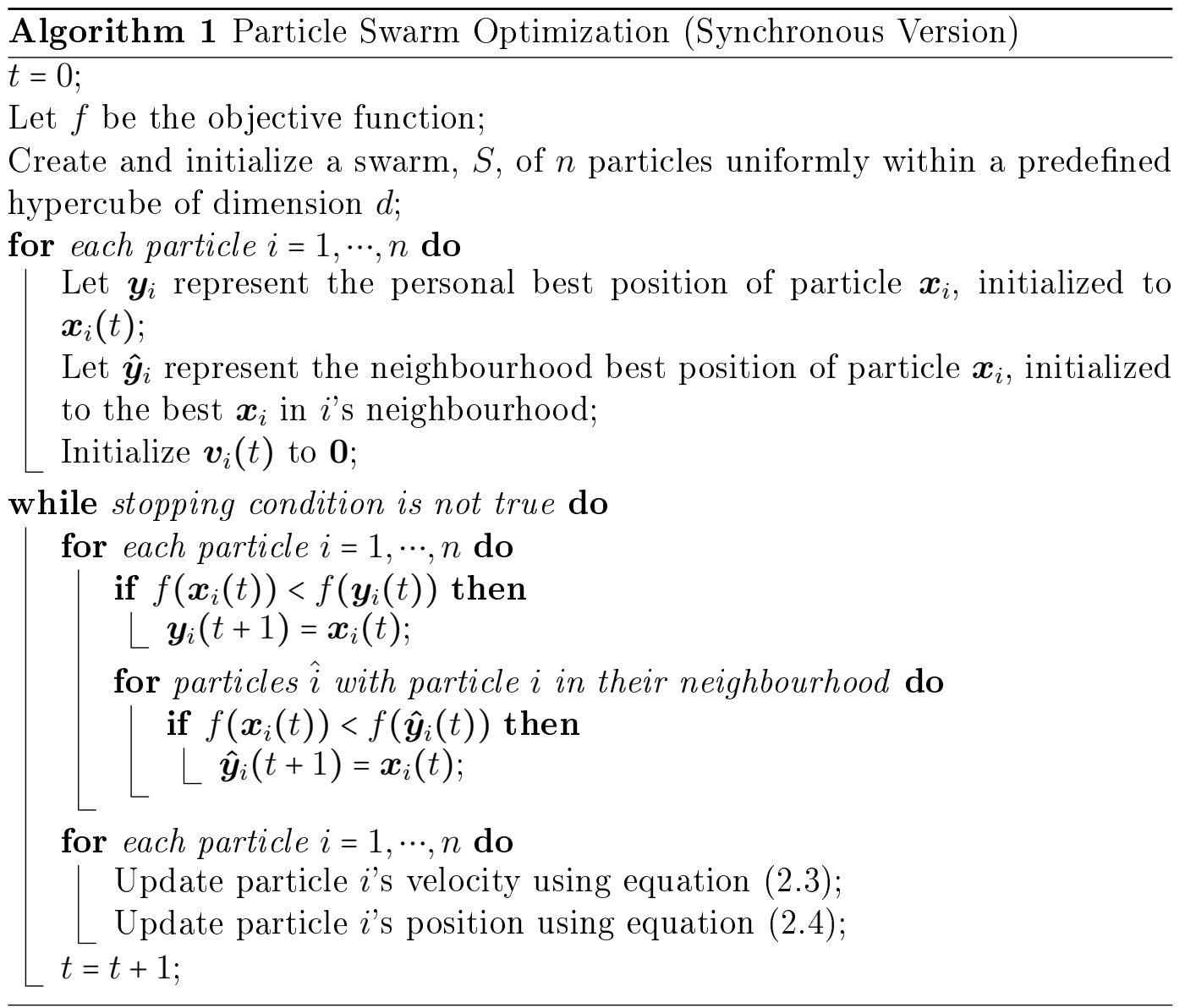

\subsubsection{Network Topologies}

As mentioned above, particles exchange information about their best positions through neighbourhoods formed by network topologies. A local network topology, such as the one in Figure 2.1a, partitions the swarm into smaller, local neighbourhoods. In this case, each particle has two neighbours. The star topology, Figure 2.1b, creates a global neighbourhood where each particle is connected to all other particles. Engelbrecht showed, through empirical evidence, that there is no significant difference in performance between PSO over a large number of benchmark problems that uses the star topology (i.e. the global-best PSO) and PSO that uses the ring topology ring (i.e. the local-best PSO). The best topology to use is, however, sometimes problem-dependent 51 . 


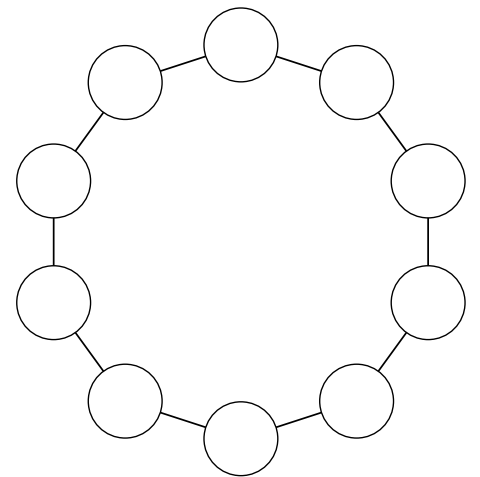

(a) 2-neighbour ring network topology

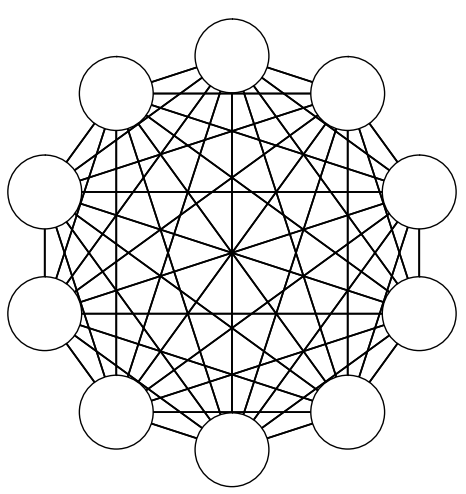

(b) Global-neighbour star topology

Figure 2.1: Network topologies

\subsubsection{Stability Conditions}

Cleghorn and Engelbrecht derived theoretical stability conditions on the PSO control parameters to guarantee order-1 and order-2 stability [33]:

$$
0<c_{1}+c_{2}<\frac{24\left(1-w^{2}\right)}{7-5 w} \text { and }|w|<1
$$

Note that stability refers to, in the case of PSO, that the velocity of each particle becomes zero.

\subsection{Multi-guide Particle Swarm Optimization}

PSO was initially developed to solve single-objective optimization problems, but over the years researchers have progressively adapted PSO to solve MOOPs. As a result, many multi-objective PSO algorithms have been published [1], [34, [36], |78], |106], [125], [143], [145], [152], [156], [169], [170], |190]. One such multi-objective algorithm is the MGPSO algorithm recently proposed by Scheepers et al [152]. The MGPSO was inspired by vector evaluated particle swarm optimization (VEPSO) in that MGPSO also utilizes multiple swarms to find solutions to MOOPs [143]. However, unlike the VEPSO, the MGPSO uses an archive to share non-dominated solutions between sub-swarms. MGPSO is highly competitive to the current state of the art PSO-based and evolutionary-based multiple objective optimization algorithms [152]; namely, speed-constrained multi-objective particle swarm optimization (SMPSO), optimized multi-objective particle swarm optimization (OMOPSO), VEPSO, nondominated sorting genetic algorithm II (NSGA II), strength Pareto evolutionary algorithm 2 (SPEA2), Pareto envelope-based selection algorithm II (PESA-II) and multi-objective evolutionary algorithm based on decomposition $(\mathrm{MOEA} / \mathrm{D})$. 
Section 2.3.1 describes the MGPSO search process. The archive management strategy used is discussed in Section 2.3.2. Section 2.3.3 presents the stability conditions on the MGPSO control parameters.

\subsubsection{Search Process}

For an $m$-objective optimization problem, the MGPSO uses $m$ sub-swarms, where each sub-swarm optimizes one of the objective functions. Non-dominated solutions found by the sub-swarms are added to a shared archive. Similar to the cognitive and social guides, MGPSO adds an archive guide to the velocity update equation thereby attracting particles to non-dominated solutions found within the archive. Velocity is updated using

$$
\begin{aligned}
\boldsymbol{v}_{i}(t+1) & =w \boldsymbol{v}_{i}(t)+c_{1} \boldsymbol{r}_{1}(t)\left(\boldsymbol{y}_{i}(t)-\boldsymbol{x}_{i}(t)\right) \\
& +\lambda_{i} c_{2} \boldsymbol{r}_{2}(t)\left(\hat{\boldsymbol{y}}_{i}(t)-\boldsymbol{x}_{i}(t)\right) \\
& +\left(1-\lambda_{i}\right) c_{3} \boldsymbol{r}_{3}(t)\left(\hat{\boldsymbol{a}}_{i}(t)-\boldsymbol{x}_{i}(t)\right)
\end{aligned}
$$

where $\boldsymbol{r}_{1}, \boldsymbol{r}_{2}$ and $\boldsymbol{r}_{3}$ are vectors of random values sampled from a uniform distribution in $[0,1] ; c_{1}, c_{2}$ and $c_{3}$ are positive constants referred to as the cognitive, social and archive acceleration coefficients respectively; $\boldsymbol{y}_{i}$ is the personal best position of particle $i ; \hat{\boldsymbol{y}}_{i}$ is the best position within the particle's neighbourhood; $\hat{\boldsymbol{a}}_{i}$ is the archive guide for particle $i$ and $\lambda_{i}$ is the archive balance coefficient.

The archive balance coefficient balances the influence of the archive and that of the social component. Smaller values of $\lambda_{i}$ will exploit the archive guide while proportionally decreasing the influence of the social guide. Similarly, larger values of $\lambda_{i}$ encourage the social guide while discouraging the archive guide. The MGPSO initializes $\lambda_{i}$ for each particle by randomly selecting a value from a uniform distribution in the range $[0,1][152$. The sampled $\lambda_{i}$ values remain static throughout the duration of the search. Random $\lambda_{i}$ values have been shown to increase convergence to the POF while maintaining diversity in non-dominated solutions 152 .

The archive guide is the least crowded non-dominated solution selected from a randomly created tournament of archive solutions. Crowding distance is used to determine the least crowded solution [41. Crowding distance is the average distance between a point in the objective space and it's nearest neighbours. Scheepers et al. found through empirical analysis that tournament sizes of two and three yielded good results [152. The least crowded solution will facilitate a more diverse Pareto optimal front, because the MGPSO will focus more on sparsely populated areas of the objective space. Algorithm 2 contains pseudo code for the MGPSO. 


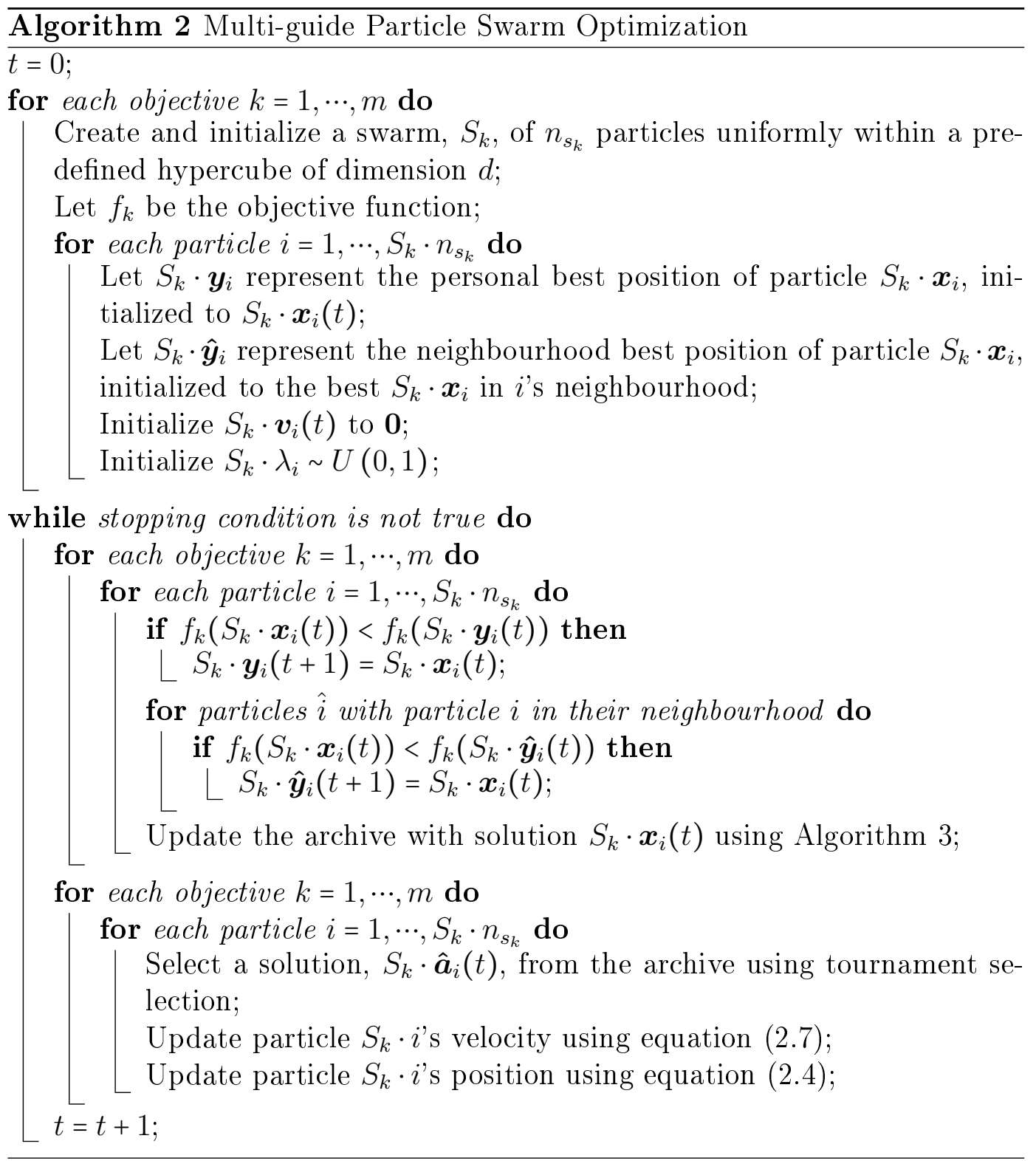

\subsubsection{Archive Management}

The original MGPSO was implemented by setting the archive's maximum capacity to the number of particles within the search space. However, any archive size - even unbounded, can be used. This number does not change over time. Once a non-dominated solution is found, and it is not dominated by any other solution in the archive using the dominance relation, it is inserted into the archive. If any solutions in the archive became dominated by the newly inserted solution, then they are removed. If the archive is full, crowding distance is used to identify the most crowded solution in the archive [41]. The most crowded solution is then removed due to the fact that the surrounding non- 
dominated solutions are similar. Algorithm 3 summarises the insert policy.

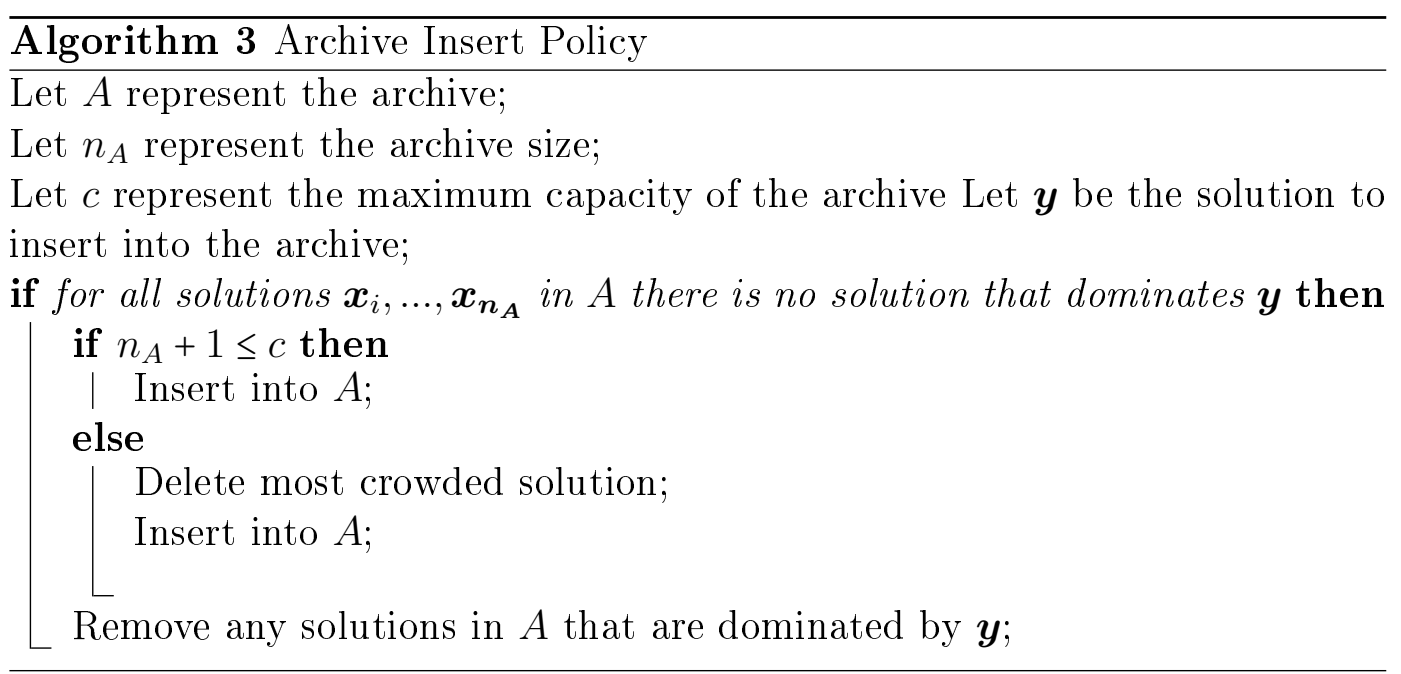

\subsubsection{Stability Conditions}

Scheepers et al. derived theoretical stability conditions on the MGPSO control parameters to guarantee order-1 and order-2 stability [152]:

$$
0<c_{1}+\lambda c_{2}+(1-\lambda) c_{3}<\frac{4\left(1-w^{2}\right)}{1-w+\frac{\left(c_{1}^{2}+\lambda^{2} c_{2}^{2}+(1-\lambda)^{2} c_{3}^{2}\right)(1+w)}{3\left(c_{1}+\lambda c_{2}+(1-\lambda) c_{3}\right)^{2}}},|w|<1
$$

If control parameter values satisfy the above stability conditions, then the swarm is guaranteed to reach an equilibrium state.

\subsection{Set-based Particle Swarm Optimization}

PSO and MGPSO were designed to solve continuous-valued optimization problems. However, there are many real-world optimization problems that do not have continuous-valued decision variables, e.g. feature selection problems, assignment problems and scheduling problems. The set-based PSO (SBPSO) algorithm combines PSO and set theory to find solutions to combinatorial optimization problems where solutions can be represented as sets [104]. SBPSO uses sets to represent particle positions, which allows for positions (i.e. solutions) of varying size. This aids in addressing the curse of dimensionality problem.

Section 2.4.1 discusses the motivation for a generally applicable, functioning set-based PSO algorithm and introduces SBPSO. The set-based operators used to implement SBPSO are described in Section 2.4.2, and Section 2.4.3 describes the SBPSO search process. 


\subsubsection{Introduction}

Several variations of PSO have been developed to solve discrete-valued optimization problems. The first such algorithm was proposed by Kennedy and Eberhart, namely binary particle swarm optimization (BPSO) [95]. A particle's position is represented as a binary string, while its velocity exists in continuous space. Velocities are transformed to respect the probability that a decision variable is either 1 or 0 . Shen et al. proposed a modified binary particle swarm optimization (MBPSO) algorithm which introduced velocity update rules designed to increase the algorithm's exploitation ability at the beginning of the search as well as the exploration of the local area around a particle's position 25. To avoid convergence to a local optimum, a fraction (that decreases over time) of the swarm was randomly reinitialized. Wang et al. proposed probability binary particle swarm optimization (PBPSO), which used a linear function to transform particles' positions to bit-valued vectors [179|. Chuang et al. introduced the catfish particle swarm optimization (CFPSO) algorithm, which was inspired by the practice of Norwegian fishermen to introduce catfish predators into tanks of captured sardines in order keep the sardines moving $|30|$. CFPSO replaces the worst performing particles in the swarm with new randomly generated particles, provided that the global best position has not changed for a given time. In a later publication, the authors applied the catfish effect to BPSO, creating a new algorithm referred to as catfish binary particle swarm optimization (CFBPSO) [31].

PSO algorithms using sets have also been previously proposed for discrete optimization problems. However, these algorithms are problem-specific, for example the algorithms proposed by Correa et al. [39], and Bock and Hettenhausen [13]. A generic set-based implementation of PSO was proposed by Veenhuis, where the positions and velocities of particles were represented as sets [177]. The algorithm used a clustering mechanism to avoid the particle positions from becoming too large, requiring a set-based distance metric. However, mathematical sets do not support the concept of distance as they lack spatial structure. Thus, Veenhuis used a problem-specific distance measure, erasing generality of the algorithm. Neethling and Engelbrecht proposed SetPSO for RNA structure prediction [127]. The algorithm represented a particle's position as a set and used probabilities to determine which elements to add to or remove from a particle. However, SetPSO was shown to perform worse for the RNA problem than other PSO approaches to discrete optimization problems, and could not be considered generally applicable [105]. Khan and Engelbrecht proposed fuzzy particle swarm optimization (FPSO) to optimize the topology design of distributed local area networks [97|. The algorithm used a fuzzy operator, the unified And-Or operator, to aggregate the multiple objectives in topology design. However, the fixed size of the FPSO particle positions meant that FPSO was not generally applicable.

Thus, there was a clear need for a truly problem-independent set-based 
PSO algorithm. Langeveld and Engelbrecht created a generally applicable, functioning set-based PSO, namely set-based particle swarm optimization (SBPSO) [104]. Research has shown that SBPSO is a highly competitive algorithm for discrete optimization problems, such as the multi-dimensional knapsack problem and the feature selection problem [104], 105]. Furthermore, SBPSO has successfully been applied to training support vector machines [128 and polynomial approximation 196 .

\subsubsection{Set-based Operators}

Formally, a set-particle's position, $X_{i}$, is defined as $X_{i} \in \mathcal{P}(U)$, where $\mathcal{P}$ is the power set of $U$ and $U$ is the universe of all elements with regard to a specific problem domain. A set-particle's velocity is represented as a set of operator pairs that either add or remove elements to or from a set-particle's position. An operation pair is denoted as $( \pm, e)$, where $(+, e)$ denotes the addition of element $e \in U$ to a particle position, and $(-, e)$ represents the removal of element $e$. The following set-operators are used to implement the velocity and position update equations of SBPSO:

Addition of two velocities, $V_{1} \oplus V_{2}$, is implemented as the union of the two sets of operation pairs:

$$
\begin{gathered}
\oplus: \mathcal{P}(\{+,-\} \times U)^{2} \rightarrow \mathcal{P}(\{+,-\} \times U) \\
V_{1} \oplus V_{2}=V_{1} \cup V_{2}
\end{gathered}
$$

Difference between two positions, $X_{1} \ominus X_{2}$, is defined as a set of operation pairs that indicate the steps required to convert $X_{2}$ into $X_{1}$ :

$$
\begin{gathered}
\ominus: \mathcal{P}(U)^{2} \rightarrow \mathcal{P}(\{+,-\} \times U) \\
X_{1} \ominus X_{2}=\left(\{+\} \times\left(X_{1} \backslash X_{2}\right)\right) \cup\left(\{-\} \times\left(X_{2} \backslash X_{1}\right)\right)
\end{gathered}
$$

Multiplication of a velocity by a scalar is implemented as

$$
\begin{gathered}
\otimes:[0,1] \times \mathcal{P}(\{+,-\} \times U) \rightarrow \mathcal{P}(\{+,-\} \times U) \\
\eta \otimes V=B \subseteq V
\end{gathered}
$$

where $B$ is a set of $\lfloor\eta \times|V|\rfloor$ elements randomly selected from $V$.

Addition of a velocity and a position is defined as the application of the velocity function $V$ to the position $X$ :

$$
\begin{gathered}
X \boxplus V: \mathcal{P}(U) \times \mathcal{P}(\{+,-\} \times U) \rightarrow \mathcal{P}(U) \\
X \boxplus V=V(X)
\end{gathered}
$$




\section{Removal operator:}

$$
\begin{gathered}
\odot^{-}:[0,|S|] \times \mathcal{P}(U) \rightarrow \mathcal{P}(\{+,-\} \times U) \\
\beta \odot^{-} S=\{-\} \times\left(\frac{N_{\beta, S}}{|S|} \otimes S\right)
\end{gathered}
$$

where $S$ is shorthand for $X(t) \cup Y(t) \cup \hat{Y}(t)$, and $Y(t)$ and $\hat{Y}(t)$ are the cognitive and social guides, respectively. $N_{\beta, S}$ is defined as

$$
N_{\beta, S}=\min \left\{|X|,\lfloor\beta\rfloor+\mathbb{1}_{\beta}\right\}
$$

with

$$
\mathbb{I}_{\beta}= \begin{cases}1 & \text { if } r<\beta-\lfloor\beta\rfloor \\ 0 & \text { otherwise }\end{cases}
$$

where $\beta$ is an element of $\mathbb{R}^{+}$and $r$ is a uniformly randomly sampled value from $(0,1)$.

\section{Addition operator to facilitate exploration:}

$$
\begin{gathered}
\odot^{+}:[0,|A|] \times \mathcal{P}(U) \rightarrow \mathcal{P}(\{+,-\} \times U) \\
\beta \odot_{\tau}^{+} A=\{+\} \times \tau-\text { Tournament Selection }\left(\mathrm{A}, \mathrm{N}_{\beta, A}\right)
\end{gathered}
$$

where $A$ is shorthand for $U \backslash(X(t) \cup Y(t) \cup \hat{Y}(t))$ and $\tau$ is the number of elements in the tournament. The tournament selection is repeated $N_{\beta, A}$ times. Thus, $N_{\beta, A}$ elements are added to $X(t)$. The element that yields the best objective function value is selected as the winner of the tournament.

\subsubsection{Search Process}

SBPSO uses the set-operators defined above to move particles through the search space. Formally, the velocity update of SBPSO is expressed as

$$
\begin{aligned}
V_{i}(t+1) & =c_{1} r_{1}(t) \otimes\left(Y_{i}(t) \ominus X_{i}(t)\right) \\
& \oplus c_{2} r_{2}(t) \otimes\left(\hat{Y}_{i}(t) \ominus X_{i}(t)\right) \\
& \oplus c_{3} r_{3}(t) \odot_{k}^{+} A_{i}(t) \\
& \oplus c_{4} r_{4}(t) \odot^{-} S_{i}(t)
\end{aligned}
$$

where $r_{1}, r_{2}, r_{3}$, and $r_{4}$ are random values, each sampled from a uniform distribution in the range $[0,1] ; c_{1}$ and $c_{2}$ are positive constants referred to as the cognitive and social acceleration coefficients, respectively; and $c_{3}$ and $c_{4}$ are positive acceleration constants for the addition and removal of elements, respectively. The position update is then the application of the velocity to the position, as shown in the following equation:

$$
X_{i}(t+1)=X_{i}(t) \boxplus V_{i}(t+1)
$$


Algorithm 4 contains pseudo code for SBPSO.

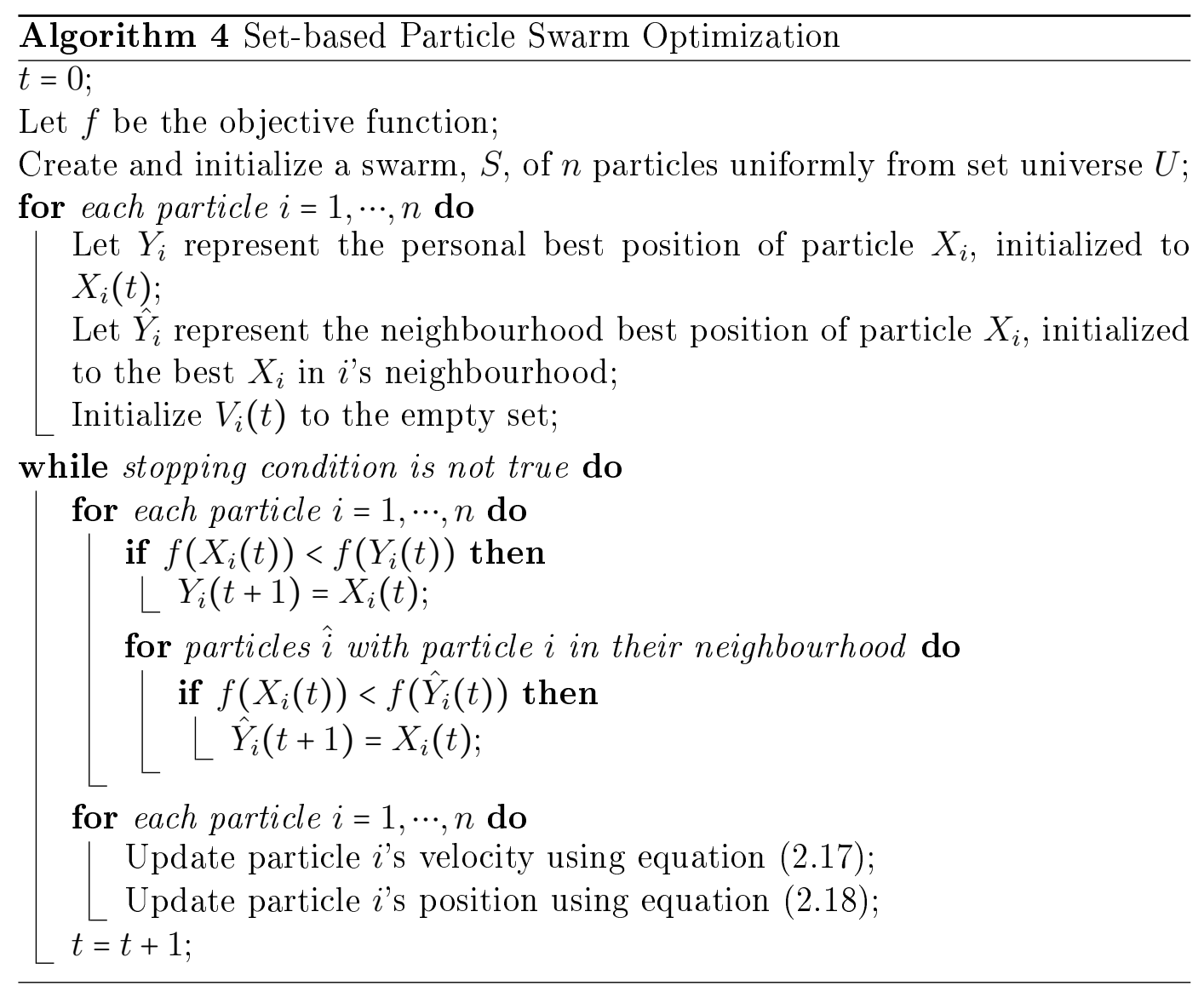

\subsection{Summary}

This chapter gave formal definitions of the optimization problems considered in this thesis. Furthermore, the necessary background on particle swarm optimization (PSO), multi-guide particle swarm optimization (MGPSO) and setbased particle swarm optimization (SBPSO) was also given. PSO and SBPSO are used in Chapters 4 and 6 to find solutions to the single-objective portfolio optimization problem. Multi-objective portfolio optimization is considered in Chapter 7, where MGPSO and SBPSO are used to obtain sets of nondominated solutions. An investigation into the performance of MGPSO using random control parameter values that satisfy stability conditions in comparison with optimized control parameters is also undertaken in Chapter 7. 


\section{Chapter 3}

\section{Portfolio Optimization}

Portfolio optimization is the process of determining which assets to invest in and how much should be invested in each asset, while considering various constraints and competing objectives. Due to the complexity of this problem, researchers have turned to meta-heuristic approaches, like evolutionary and swarm intelligence, for their simplicity and ability to approximate optimal solutions efficiently. This chapter ${ }^{1}$ provides a comprehensive review of over 100 publications that have applied evolutionary and swarm intelligence algorithms to portfolio optimization. The publications discussed span over decades, from 1993 to 2019, and are categorized by whether constraints are considered, as well as if a single or multi-objective algorithm was used. This also includes a variety of different sub-classifications of evolutionary and swarm intelligence algorithms. Moreover, all publications considered portfolio optimization as a static optimization problem. Other characteristics of the publications such as commonly considered constraints, unique constraints, objectives, portfolio models, benchmarks and performance measures are also given.

The remainder of this chapter is organized as follows: Section 3.1 familiarises the reader with portfolio optimization and its concepts. Section 3.2 introduces the evolutionary and swarm intelligence algorithms found in the reviewed publications. Sections 3.3 and 3.4 discuss unconstrained and constrained portfolio optimization, respectively. The reviewed literature is analyzed in Section 3.5. Section 3.6 summarises the findings of the analysis and concludes the chapter.

\subsection{Introduction}

Investing is an effective way to put money to work and potentially build wealth. An investment is the exchange of currently available resources for assets in the expectation of a future gain. However, investing does not guarantee that one's

\footnotetext{
${ }^{1}$ This chapter is currently being reviewed for the journal Applied Soft Computing under the title "Evolutionary and swarm intelligence algorithms for portfolio optimization" |58.
} 
wealth will simply accumulate over time since the value of an investment often rises and falls. It is near impossible to predict exactly when these rises and falls in value will occur and as such, there is a level of risk associated with each asset. Formally, risk is the degree of uncertainty regarding the evaluation of an asset either increasing or decreasing in value. Naturally, the riskiness of an asset will change over time as it is affected by ongoing internal and external factors, such as unfavorable news and bear markets, respectively [68]. The risk, in some cases, might be worthwhile as an asset's value increases, exceeding expectations. It is very common for investors to have a stake in multiple assets. These assets are collectively referred to as a portfolio. Investors are, thus, continuously tasked with making investment decisions that maximize the profitability of their portfolio while simultaneously minimizing its risk exposure.

Risk can be mitigated through the strategic diversification of a portfolio through the selection of uncorrelated assets [68], [69]. The premise of the strategy is that the riskiness of an asset imposed on the portfolio can be negated by selecting an unrelated asset. For example, if one asset experiences losses, the gains of another will compensate. As the range of assets within a portfolio widens, so does the effectiveness of diversification. However, the riskiness and profitability of a portfolio are not set in stone, and as time passes these aspects of the portfolio will shift. For example, an asset in what was an equally weighted portfolio may have increased significantly, resulting in the other assets contributing less to the overall value of the portfolio. An investor will then have to re-balance the portfolio in order to realign it with their goals.

Choosing which assets to include in a portfolio that will result in an optimal trade-off between return and risk is a non-trivial task. This section presents the necessary background for the work presented in this chapter as well as for concepts discussed in other chapters. Section 3.1.1 introduces one of the most prominent portfolio optimization models, the mean-variance model. Additional constraints and objectives are presented in Section 3.1 .2 and Section 3.1.3. respectively. Section 3.1.4 briefly discusses alternative portfolio optimization models.

\subsubsection{History}

In 1952, Markowitz, one of the founders of modern portfolio theory, presented a portfolio optimization model, known as the mean-variance model, in an effort to construct portfolios in such a way that the total profit of the portfolio is maximized subject to an acceptable level of risk [115], [164]. Markowitz's model would go on to earn him a Nobel Prize in Economic Sciences. Meanvariance is used to determine a portfolio that represents an optimal trade-off between the risk and returns. The quadratic programming model is formulated as follows:

$$
\text { minimize } \lambda \bar{\sigma}-(1-\lambda) R
$$


where $\bar{\sigma}$ is the total risk of the portfolio, $R$ is the total return of the portfolio, and $\lambda$ represents an investor's risk tolerance in the range $[0,1]$. Return is maximized irrespective of the risk involved when $\lambda$ is equal to zero. $\mathrm{A} \lambda$ value of one minimizes risk regardless of profits. An explicit trade-off between risk and return is represented by a $\lambda$ value less than 0 and greater than 1 . A diverse set of optimal portfolios can, therefore, be obtained by solving equation (3.1) for varying values of $\lambda$. Risk is calculated using

$$
\bar{\sigma}=\sum_{i=1}^{n} \sum_{j=1}^{n} w_{i} w_{j} \sigma_{i j}
$$

where $n$ is the number of assets, $w_{i}$ and $w_{j}$ is a weighting of assets $i$ and $j$, respectively, and $\sigma_{i j}$ is the covariance between assets $i$ and $j . R$ is calculated using

$$
R=\sum_{i=1}^{n} R_{i} w_{i}
$$

where $R_{i}$ is the return of asset $i$. Equation (3.1) requires that the total budget of the portfolio is distributed according to each asset. This constraint, referred to as the budget constraint, is expressed as

$$
\sum_{i=1}^{n} w_{i}=1
$$

Furthermore, the weight of each asset must be non-negative, i.e.

$$
w_{i} \geq 0
$$

\subsubsection{Constraints}

The traditional mean-variance model does not take into account many of the constraints that portfolio managers must adhere to. As a result, many constrained adaptations have been proposed. These constraints significantly increase the complexity of portfolio management. The most commonly considered constraints for portfolio optimization are discussed below.

Cardinality restrains a portfolio by limiting the total number of assets included. The cardinality constraint is given as

$$
\sum_{i=1}^{n} z_{i}=K
$$

where $z_{i}$ is equal to 1 if asset $i$ is included in the portfolio, and $K$ represents the desired number of assets to be included [18]. Alternatively, the constraint can be represented as an inequality, i.e.

$$
K_{\min } \leq \sum_{i=1}^{n} z_{i} \leq K_{\max }
$$


where $K_{\min }$ and $K_{\max }$ represent the minimum and maximum number of assets, respectively [26], [27], [42], [108], [122], [158].

Floor and ceiling, also known as the boundary constraints, represent the upper and lower bounds on the asset weights, respectively. This guarantees that an asset does not become too risky by over-representation or insignificant, by under-representation. The floor and ceiling constraints are defined as

$$
0 \leq \varepsilon \leq w_{i} \leq \delta \leq 1
$$

where $\varepsilon$ and $\delta$ are the upper and lower bounds, respectively [18]. When floor, ceiling and cardinality constraints are imposed on equation $(\sqrt{3.1)}$, it is referred to as cardinality-constrained portfolio optimization [18].

The minimum turnover constraint, which ensures that a portfolio must meet a given level of profitability, is enforced as

$$
\sum_{i=1}^{n} w_{i} R_{i} \geq R^{*}
$$

where $R^{\star}$ is the minimum turnover [19, [151, 159, 183.

Liquidity represents how easily an asset can be converted into other types of equity, such as cash. The total liquidity of a portfolio can be constrained to meet a minimum liquidity threshold. The liquidity constraint is defined as

$$
\sum_{i=1}^{n} w_{i} e_{i} \geq e^{*}
$$

where $e^{*}$ is the minimum amount of liquidity and $e_{i}$ is the liquidity of asset $i$ [60], [131].

Transaction lots refer to the number of units of an asset that can be traded at a time. The number of units held of an asset must be a multiple of the specified transaction lot. Thus, the contribution weight of an asset is determined by the number of lots for an asset held in proportion the total number of lots held. Formally, the constraint is defined as

$$
w_{i}=\frac{l_{i} q_{i}}{\sum_{j=1}^{n} l_{j} q_{j}}
$$

where $l$ and $q$ are the transaction lot and quantity of lots held, respectively, for assets $i$ and $j$ 67, ,70, 159.

Transaction costs are paid to an entity, such as an exchange, when buying or selling an asset. Transaction costs typically occur when rebalancing a 
portfolio. Transaction costs are imposed as

$$
\sum_{i=1}^{n} w_{i} c_{i} \leq B
$$

where $c_{i}$ is the cost of asset $i$ and $B$ is the budget [23], [24], [28], |70], |147], 159.

Capitalization refers to the total market value of a company's outstanding shares. Thus, sector capitalization is the capitalization of a sector, where a sector represents a part of the economy containing assets which offer similar goods or services. The sector capitalization constraint ensures that if an asset or assets representing a sector is included, then that sector should occupy a portion of the portfolio larger than a sector with a lower capitalization ranking. Formally, the constraint is defined as

$$
\sum_{i=1}^{n_{s^{\prime}}} w_{s^{\prime}, i}<\sum_{i=1}^{n_{s}} w_{s, i}
$$

where $n_{s^{\prime}}$ and $n_{s}$ are the number of assets in sectors $s^{\prime}$ and $s$, respectively, and $w_{s^{\prime}, i}$ and $w_{s, i}$ are the asset weights of sectors $s^{\prime}$ and $s$, respectively. [67], [159].

\subsubsection{Objectives}

Many extensions to the mean-variance model have been proposed since its initial outline. Often these extensions include constraints, but also objectives. For example, maximizing the diversity of the types of assets included in the portfolio. Furthermore, the previously mentioned constraints can be reformulated as objectives, such as minimizing cardinality [2] and maximizing liquidity [192. Portfolio optimization can also be viewed as a multi-objective optimization problem. Quite simply, equation (3.1) can be reformulated where $\bar{\sigma}$ and $R$ are opposing objectives to be minimized and maximized, respectively. Thus, a set of non-dominated solutions can be obtained as opposed to optimizing for a given $\lambda$ value.

\subsubsection{Alternative models}

Despite the success of the mean-variance model, it is not without its flaws. It has been criticized as being unrealistic, often leading to unsatisfactory predictions of investor behaviour [164], particularly in cases where the distribution of returns is asymmetric [66], [164]. This has led to the formulation of alternative portfolio optimization models. Often these alternative models make use of different definitions of $\bar{\sigma}$ and $R$ or completely different objectives. One such approach by Markowitz is to use semi-variance, which measures the downside part of the return distribution as risk 164. Semi-variance has been regarded 
as a more appropriate $\bar{\sigma}$ measure than covariance, in the sense that it reflects an investor's intuition about risk. Using semi-variance, $\bar{\sigma}$ is redefined as

$$
\bar{\sigma}=\frac{1}{n} \sum_{i=1, R_{i}<C}^{n}\left(R_{i}-C\right)^{2}
$$

where $C$ is a benchmark, i.e. the average return over a period of time. However, deciding which benchmark to use is a common problem as different benchmarks could lead to different outcomes in results. $R$ is then formulated as

$$
R=\sum_{t=1}^{T} \sum_{i=1}^{n} w_{i} \frac{R_{i}}{T}
$$

where $T$ is the time period considered. Following equation (3.1), these definitions are used to create the mean-semivariance model. Other portfolio optimization models include, but is not limited to, value-at-risk (VaR) 108, [116], conditional value-at-risk (CVaR) [108], [116], prospect theory [110], [129], Sharpe ratio [5], [194], and mean absolute deviation (MAD) [19].

\subsection{Evolutionary and Swarm Intelligence Algorithms}

Evolutionary and swarm intelligence algorithms are stochastic populationbased search algorithms inspired by naturally occurring phenomena. Many adaptations of such algorithms have been applied to single and multi-objective portfolio optimization. The original algorithms which inspired these adaptations are presented below to convey a general understanding of the ideas expressed by each algorithm. Section 3.2.1 discusses evolutionary algorithms (EAs) and 3.2.2 discusses swarm intelligence (SI).

\subsubsection{Evolutionary Algorithms}

Biological evolution is the optimization of an organism's ability to survive in dynamically changing and competitive environments $[52 \mid$. EAs are inspired by this process and mimic its characteristics in order to seek global optima for computational optimization problems. The survival strength of individuals within a population is determined by the objective function to be optimized. Individuals are then selected based on their survival strength for reproduction, which produces offspring for the next generation of individuals. The following algorithms belong to the EA paradigm:

Differential evolution (DE) is an EA that uses distance and direction information from the current population to guide the search process [161]. 
Genetic algorithms (GAs) are inspired by Darwin's theory of natural selection [52], [85]. GAs simulate genetic evolution by representing individuals as collections of genotypes, where each genotype corresponds to a characteristic of the objective function.

\subsubsection{Swarm Intelligence}

SI is defined as the cooperation of individuals within a group to achieve an objective by exchanging locally available information [52. PSO and its adaptions seen in Chapter 2 are examples of SI algorithms. The following are also SI based algorithms:

Ant colony optimization (ACO) refers to a collection of SI algorithms that model the foraging behaviour of ants [47]. Ants communicate indirectly with each other by releasing pheromones that slowly evaporate. Over time, all ants follow the trail/path with highest pheromone concentration.

Artificial bee colony (ABC) is an optimization algorithm based on the foraging behaviour of honey bees [92]. The colony is guided towards global optima based on the quantity of nectar found at various food sources located throughout the search space. Three types of artificial bees are used to perform the search process, each type having a particular role. Employed bees exploit the aforementioned food sources and share this information with the colony via a waggle dance. Onlooker bees watch this waggle dance and adjust their direction according to the quality of nectar. When all known food sources have been adequately exploited, scout bees search for new sources of nectar.

Bacterial foraging algorithm (BFA) mimics the foraging behaviour of Escherichia coli (E.coli) by implementing chemotaxis, reproduction, swarming and elimination-dispersal processes to search for optima [111]. E.coli are cooperative-foraging bacteria present in human intestines that form highly structured colonies around nutrient-rich environments. Furthermore, E.coli abandon nutrient-poor regions and actively avoid harm.

Bat algorithm (BA) mimics the foraging behaviour of bats, which use echolocation for avoidance of obstacles and detection of potential prey [186].

Cat swarm optimization (CSO) models the hunting behaviour of felines to find optimal solutions [29]. During the search, cats switch between two modes, seeking and tracing. Cats in seeking mode rest while analyzing their environment for the next best move. Tracing cats move in the direction of their velocity and are influenced by the position of their leader.

Chemical reaction optimization (CRO) models the phenomenon in which 
products produced by the microscopic process of molecules undergoing chemical reaction are always more stable than the reactants [103].

Firefly algorithm (FA) models the flashing-based interactions between fireflies to seek global optima 187]. The following principles are used to guide the search process. Firstly, the brightness of a firefly represents the attractiveness of a solution. Secondly, a less bright firefly moves towards a brighter one. Lastly, brightness increases as the distance between fireflies decreases.

Fireworks algorithm (FWA) mimics the explosion of fireworks and the subsequent sparks produced by the fireworks [168]. The quantity and amplitude of sparks produced is determined by the closeness of a firework to the worst and best location (firework) found thus far, respectively. Fireworks closer to the worst performing firework produce less sparks. Fireworks closer to the best firework produce small explosions to act as a form of local search. Furthermore, sparks are randomly generated as a means of maintaining diversity.

Krill Herd (KH) is a biologically-inspired algorithm based on the simulation of the social and foraging behaviour of krill. The movement of krill is influenced by the density of the herd, distance from food, and random diffusion [65].

Invasive weed optimization (IWO) utilizes artificial colonies of weeds, that are in competition for survival, to seek global optima. Weeds are aggressive and invasive with respect to their growth behaviour. Furthermore, weeds have been shown to be robust and capable of adapting to change in the environment. These properties form the basis of IWO [117]. The colonies move through the search space by spreading seeds, where fitter individuals produce more seeds.

\subsection{Unconstrained Portfolio Optimization}

Chang et al. introduced the concept of unconstrained portfolio optimization which considered the mean-variance model [115], [164] without any additional constraints [18]. Thus, unconstrained portfolio optimization must only adhere to the budget and positive weight constraints defined in equations (3.4) and (3.5), respectively. The term unconstrained portfolio optimization has since grown to include other portfolio models without any additional constraints. This section presents single-objective algorithms for unconstrained portfolio optimization in Section 3.3.1 and multi-objective algorithms for unconstrained multi-objective portfolio optimization in Section 3.3.2. 


\subsubsection{Single-objective Algorithms}

\section{Ant Colony Optimization}

Zhu et al. showed that ACO is capable of delivering similar performance to that of PSO for the unconstrained portfolio optimization problem with varying sized portfolios 193 .

\section{Bacterial Foraging Optimization}

Niu et al. developed a variant of BFO with a linearly decreasing chemotaxis step length that was shown to be highly competitive amongst other algorithms, namely PSO and GA 131.

\section{Genetic Algorithms}

GAs have been widely used for unconstrained portfolio optimization. Arnone et al. applied a multi-species GA to a semi-variance trade-off based portfolio model where each species optimized the objective function for a different $\lambda$ value |7|. Shoaf and Foster showed that a GA, as well as a multi-species implementation of a GA, found more good-quality portfolios than quadratic programming [154], [155]. Chang et al. showed that a GA was capable of finding higher quality portfolios than simulated annealing (SA) and tabu search (TS) [18. Busetti confirmed GA's superiority over TS, especially for larger problems $[15]$. The performance of the GA was further improved by utilizing a two-step procedure that identified optimal assets beforehand [17]. The choice of control parameter values for GA, specifically applied to portfolio optimization, was empirically shown to have an effect on performance [76. Likewise, the encoding of solutions has also been shown to have an impact on the ability of heuristics to identify optimal solutions. An example of an encoding which leads to superior solutions than that of an array-based encoding is to use a tree-based representation [5].

\section{Particle Swarm Optimization}

PSO has been shown to be very competitive amongst other approaches to unconstrained portfolio optimization. Kamali showed that PSO was able to obtain higher quality portfolios in less time than a GA |88|. PSO, compared with a GA and an industry tool, proved to be a superior algorithm in finding solutions to portfolio optimization [194|. PSO was shown to outperform a GA in terms of consistency and speed of convergence towards an optimal solution for a behavioural portfolio model [129]. Liu et al. presented a novel adaptation of PSO that was shown to be effective in finding portfolios for a multi-period portfolio model that considered behavioural aspects of investors [110|. Chen et al. found that the use of velocity clamping resulted in better portfolios 23. Niu et al. proposed a symbiotic multi-swarm PSO model that was shown to yield superior portfolios, both in quality and diversity, than a previously proposed multi-swarm cooperative PSO [132]. 


\subsubsection{Multi-objective Algorithms}

\section{Ant Colony Optimization}

Khalidji et al. proposed a multi-objective ACO algorithm that was able to obtain solutions closer to the true POF than the NSGA II [96].

\section{Genetic Algorithms}

Multi-objective GAs have also been shown to be successful approaches to unconstrained portfolio optimization. Computational analysis found the Pareto envelope-based selection algorithm (PESA) to be superior to NSGA II and SPEA2 for minimising risk (equation (3.2)) and maximizing return (equation (3.3).) [45. Chiam et al. suggested an order-based representation that was shown to lead to better performance for algorithms [26].

\section{Particle Swarm Optimization}

In a comparative study of four multi-objective optimization algorithms, namely the parallel single front genetic algorithm (PSFGA), multi-objective particle swarm optimization (MOPSO), SPEA2 and NSGA-II. MOPSO found a superior and more diverse set of portfolios [119]. In contrast, PSFGA was the worst performing algorithm. Wang and Watada made improvements to the MOPSO algorithm that were shown to be advantageous in finding a diverse POF [178]. A dynamic multi-swarm PSO consistently outperformed multi-objective differential evolution (MODE), MOPSO and NSGA-II for large scale portfolio optimization that considered 200 and 500 assets [109].

\subsection{Constrained Portfolio Optimization}

Constrained portfolio optimization was pioneered by Chang et al, laying the foundation for future work [18]. Chang et al. proposed the cardinality constrained portfolio problem (CCPP), in which the cardinality and the floor and ceiling constraints were introduced to the classic mean-variance problem. Thereafter, extended problems and other models that include further constraints have also been studied. Section 3.4.1 discusses results of singleobjective algorithms for constrained portfolio optimization and multi-objective algorithms for constrained multi-objective portfolio optimization are discussed in Section 3.4.2.

\subsubsection{Single-objective Algorithms}

\section{Ant Colony Optimization}

Deng and Lin found ACO to be more robust and effective than PSO in finding solutions to CCPPs, especially for low risk investment portfolios [43].

\section{Artificial Bee Colony}


$\mathrm{ABC}$ has been shown to be a highly competitive algorithm for the constrained portfolio optimization problem. ABC significantly outperformed local search techniques, such as SA and TS |20|. Furthermore, experimental results by Wang et al. showed that $\mathrm{ABC}$ is capable of finding higher quality solutions than PSO 181. Chen et al. proposed an improved ABC algorithm that generates scout bees by selecting previously found solutions from an efficiency frontier archive [21]. The proposed improved ABC algorithm consistently outperformed the original $\mathrm{ABC}$ algorithm. A global best guided ABC model, inspired by PSO, found a more diverse set of non-dominated solutions than other algorithms, namely GA, ABC and FA [66], [167|. Several techniques used by GAs were applied to $\mathrm{ABC}$ to produce a very competitive and efficient approach to finding optimal portfolios 87]. Hong-mei et al. showed that ABC obtained more portfolios in fewer iterations than GA [77]. ABC continuously outperformed FA and GA for portfolio optimization subject to many constraints [10]. A hybrid ABC algorithm, in which employed bees used a FA search procedure, was shown to be highly competitive amongst previously tested algorithms [18], [40], [172].

\section{Bacterial Foraging Algorithm}

Experimental results confirmed BFO to be the superior choice for portfolio optimization over other algorithms, namely GA, TS, SA [18] and PSO [40], especially for portfolios that considered a large number of assets [91.

\section{Bat Algorithm}

Computational results showed that BA, PSO and CSO yielded similar performance for the constrained portfolio optimization problem [89] [163]. Strumberger et al. showed that the performance of BA was significantly improved by introducing scout behaviour adapted from ABC.

\section{Cat Swarm Optimization}

Kamili and Riffi found CSO to be a good approach to for the constrained portfolio selection problem $|90|$.

\section{Differential Evolution}

Lwin and Qu integrated population based incremental learning with differential evolution and found that the proposed hybrid demonstrated superior performance over the individual algorithms 114. Pai and Michel used DE to solve a unique portfolio optimization problem that involved long and short positions inspired by an equity market neutral portfolio strategy [137|. A long position asset, the most familiar kind of asset, yields returns as its value increases. A short position, in contrast, yield returns expecting a fall in the asset's value. Thus, a combination of long and short assets aims to minimize risk exposure. Mathematically, short positions are represented as negative weights. Furthermore, Pai and Michel defined a risk budget, where the riskiness of each asset 
in the portfolio had to be under a given limit. Pai and Michel also applied DE, as well as a GA, to a portfolio model based on an investment strategy known as the 130-30 strategy [139|. This strategy aims to yield greater returns by short-selling poor-performing assets, 30\% of the portfolio value. Then, $130 \%$ of the portfolio value is used to purchase assets, which investors feel that would outperform the market.

\section{Chemical Reaction Optimization}

$\mathrm{Xu}$ et al. evaluated the performance of CRO for constrained portfolio optimization and proposed an improved model that found higher quality solutions than the original CRO 184 .

\section{Krill Herd}

Tabu et al. found that KH underperformed GA and FA for constrained portfolio optimization 174 .

\section{Firefly Algorithm}

Bacanin and Tabu presented several modifications to enhance the exploratory and exploitative behaviour of FA |8|, |173|. The proposed FAs were shown to be very competitive amongst previously proposed GA, TS, SA [18 and PSO [40.

\section{Fireworks Algorithm}

Tabu et al. [9] concluded that FWA performed significantly better than GA, $\mathrm{ABC}, \mathrm{KH}$ and FA. FWA found higher quality solutions in very few iterations which continued to improve throughout the search. The authors concluded that the algorithm has great potential for solving the portfolio optimization problem and that further work should be done to further evaluate the performance.

\section{Invasive Weed Optimization}

Pouya et al. proposed a constrained portfolio model where risk, market expectations and experts recommendations were considered as weighted objectives [144. The authors found that, in general, IWO and PSO demonstrated similar performance.

\section{Genetic Algorithm}

Chang et al. compared the performance of GA, SA, and TS. They found that GA only slightly outperformed SA and TS for cardinality-constrained portfolio optimization [18]. Furthermore, GA was consistently slower than the other approaches. Ehrgott et al. found similar results where GA, SA, TS and local search were used to optimize a constrained portfolio model which considered five equally-weighted sub-objectives [50]. Woodside-Oriakhi et al. used a set-based encoding which improved the performance of a GA, SA, and 
TS. Furthermore, the improved algorithms were shown to scale well to larger test problems that included up to 1318 assets [183. Chang et al. demonstrated the robustness of a GA in its ability to solve a variety of cardinalityconstrained problems [19]. Similarly, Soleimani et al. confirmed GA to be a reliable approach to optimizing small and large sized portfolios under several constraints 159. Jalot and Thakur designed an effective GA for cardinalityconstrained portfolio optimization which utilized an efficient repair mechanism [18], as well as constraint-aware crossover and mutation operators [84]. Guo et al. discussed the difficulties in optimizing a fuzzy multi-period portfolio selection model with different investment horizons [70]. As a solution to the complex portfolio model, the authors proposed a fuzzy-based GA that was shown to be efficient and effective. Lin provided empirical results that demonstrated the applicability of GA for various constrained portfolio models [28]. Pai and Michel developed a GA which used $k$-means clustering to eliminate the cardinality constraint [138. Huang and Shen used a GA to demonstrate the practicality of a multi-period portfolio model with transaction costs and fuzzy returns $[79]$. Huang showed that the GA was capable of finding optimal portfolios for fuzzy-constrained mean-variance and semi-variance models [80]. Ackora et al. showed that the GA's ability to find optimal portfolios is significantly impacted by the chosen crossover operator $|71|$. Drezewski and Doroz developed a co-evolutionary GA for constrained portfolio optimization [48]. Yu et al. presented a two-stage GA which first identified good-quality assets before optimizing the asset weights [189]. The two-stage procedure significantly improved performance in determining the most valuable portfolio. Integration of quadratic programming with GAs has shown to increase performance for constrained portfolio optimization [123], [147]-[149]. Evolutionary techniques are used to satisfy constraints while quadratic programming is used to determine optimal weights. Liu and Zhang applied a GA to a heavily constrained portfolio model that defined multiple fuzzy objectives, return, risk and liquidity, as a fuzzy single-objective optimization problem [112].

\section{Particle Swarm Optimization}

A large amount of work has considered PSO as an approach to portfolio optimization. $\mathrm{Xu}$ et al. were the first to evaluate the performance of PSO for constrained portfolio optimization [23], [61]. The authors concluded that the use of velocity clamping improved performance. PSO has been shown to achieve similar performance to those of previously tested GA, SA, and TS [18], [40]. In an effort to improve performance, significant work has been done on improving the velocity control of PSO. Koshino et al. studied several velocity management strategies and found that the use of a combination of techniques improved performance $|99|$. Likewise, Chen and Ben-yan modified the exploration and exploitation behaviour of PSO which resulted in superior performance over that of GA $[22$. Feng et al. found that the use of dynamic coefficients, particle mutation and velocity clamping made PSO a more effec- 
tive algorithm for finding optimal portfolios than previously tested algorithms [44]. Wang et al. incorporated evolutionary attributes into PSO to maintain diversity and to avoid premature convergence [180. The Hybrid PSO was found to be very competitive, producing higher quality solutions while being computationally efficient. Yaakob and Watada found that the use of mutation and velocity control bested a GA for constrained portfolio optimization problems [185|. Chen and Zhang demonstrated the versatility of PSO in finding solutions to a fuzzy variant of the mean-variance model [24]. Work by Corazza et al. found PSO to be a viable approach to constrained portfolio optimization [38]. Farzi et al. concluded that PSO under quantum motion was better suited to constrained portfolio optimization than use of classical Newtonian movements $[60 \mid$. Ni et al. proposed several dynamic and random topology strategies for PSO $[130]$. Computational results showed that topologies which progressed from small ring structures to a fully connected network resulted in higher quality portfolios. Sadigh et al. proposed a PSO and neural network hybrid that outperformed the individual algorithms, as well as previously evaluated GA, TS and SA strategies [18], [150]. Sub et al. proposed a variation of PSO which mimicked the thermal and drift motion of electrons in a conduct under an electrical field [166]. Results showed that the proposed drift-based PSO was statistically significantly better than other optimization approaches, in both computational time and quality of solutions found. The combination of PSO [191] and binary PSO [94 successfully outperformed a GA [159], especially for larger cardinality constrained portfolios. In order to prevent PSO from being trapped in local optima, Mozafari et al. [126] introduced SA behaviour to PSO [99] that found better portfolios than GA, TS, SA [18 and PSO. Work done by Corazza et al. showed PSO to be a viable approach to constrained portfolio optimization [38]. However, Salahi et al. found that PSO [40] was bested by a local harmony search technique, both in time and accuracy [151]. Moreover, the performance difference was most notable on problem instances with larger data sets.

\subsubsection{Multi-objective Algorithms}

\section{Ant Colony Optimization}

Armananzas and Lozano proposed a multi-objective ACO and greedy search hybrid which demonstrated outstanding performance over standalone greedy search [6]. Doerner et al. proposed an enriched Pareto ACO algorithm that was shown to outperform Pareto SA and NSGA-II for randomly generated portfolio problems, as well as a real world problem [46].

\section{Artificial Bee Colony}

Kumar and Mishra proposed a multi-objective ABC algorithm which used covariance principles to approximate the gradient of the objective function [100]. Empirical results showed that the proposed ABC algorithm was able to pro- 
duce a diverse POF.

\section{Bacterial Foraging Algorithm}

Mishra et al. presented a multi-objective $\mathrm{BFO}$ algorithm which was capable of identifying a diverse POF [121], [122].

\section{Differential Evolution}

Unlike other papers, Pai and Michel defined a portfolio optimization problem that considered the futures market, which is fraught with risks [140. This portfolio model defined three objectives to minimize exposure to risk in future bonds, indices, and currency markets. MODE was shown to successfully obtain practical solutions. In the same line of work, Pai introduced transaction costs and risk budgets to further mimic the real world [136|. Most recently, Pai examined the ongoing effects of rebalancing a portfolio as a multi-objective optimization problem 135 .

\section{Particle Swarm Optimization}

Zhou and Li presented an improved MOPSO algorithm that was shown to find a diverse set of solutions close to the POF for constrained portfolio optimization which considered return, variance and fuzzy-liquidity as objectives [192. Mishra et al. developed a MOPSO algorithm which used a self-regulating velocity strategy that was found to be highly competitive against evolutionary algorithms, namely NSGA-II and SPEA-2 [120]. Kaucic presented a novel hybridized MOPSO algorithm to solve the constrained portfolio optimization problem [93. The hybridized algorithm used mutation to improve the exploratory capabilities of MOPSO. Computational results confirmed the effectiveness of introducing mutation to aid MOPSO in finding optimal portfolios. Silva et al. presented a comprehensive study in which an adaptive ranking MOPSO was shown to be highly competitive against past approaches, most notably outperforming previously dominant MOEAs such as SPEA2 and NSGA-II [157.

\section{Genetic Algorithm}

SPEA2 consistently outperformed other state of the art multi-objective evolutionary algorithms (MOEAs), namely vector evaluated genetic algorithm (VEGA), multi-objective genetic algorithm (MOGA), and NSGA-II, in finding solutions close to the true POF [158. Chiam et al. found that an orderbased representation of solutions lead to improved performance [26], [27]. A comparative study by Anagnostopoulos and Mamanis found that state of the art MOEAs, in general, are efficient and reliable strategies for cardinalityconstrained portfolio optimization [3]. Specifically, PESA obtained on average solutions slightly closer to the POF, while NSGA-II and SPEA2, on average, found more diverse solutions. Similar work was performed where the cardinality constraint was treated as a third objective and represented as an 
inequality [2]. Computational analysis found SPEA2 to be the best performing algorithm whereas PESA, which placed second, was the fastest approach. Branke et al. proposed an integrated active set MOEA, referred to as the envelope multi-objective genetic algorithm (E-MOEA) [14|. E-MOEA was shown to find significantly better frontiers than NSGA-II. A study of the state of the art MOEAs as well as GA [18] applied to cardinality constrained portfolio optimization found that all MOEAs outperformed the single-objective GA [4]. Furthermore, SPEA2 provided the most reliable performance, however, at the cost of being computationally expensive. Further experiments confirmed the ability of best performing algorithms, i.e. SPEA2 and NSGA-II, to scale to larger search spaces of 2196 assets. Deb et al. proposed a hybridized NSGA-II procedure which used clustering and a local search to obtain more accurate solutions 42 . The authors demonstrated the effectiveness of the algorithm and concluded that it was a reliable approach to cardinality-constrained portfolio optimization. Liagkouras significantly improved the performance of the state of the art MOEAs by using a three-dimensional encoding that made processing time invariant to the size of the portfolio problem [108]. Liagkouras and Metaxiotis proposed a mutation operator that aggressively probes the search space to guide offspring to optimal solutions [107]. The mutation operator was shown to increase the effectiveness of NSGA-II and SPEA2 for cardinality constrained portfolio optimization, however, at the expense of being computationally expensive. Meghwani and Thakur presented an extended three-objective portfolio model that considered minimizing transaction costs as an additional objective [116]. A novel repair algorithm was proposed to handle the newly introduced transactional constraints, specifically equality constraints. MOEAs with the repair algorithm outperformed their standard counterparts. Streichert and Tanaka-Yamawaki proposed a hybridization of NSGA-II and a quadratic programming local search which demonstrated improved solution quality for some problem instances, however, suffered from premature convergence on others [162]. Subbu et al. proposed an architectural solution to the constrained portfolio optimization problem which sequences multiple techniques to produce Pareto optimal solutions [165]. Linear programming was used to generate the initial population for PESA. The efficient frontier obtained by PESA is enhanced by a GA which identifies possible gaps in the POF. Lastly, constraints are then applied to the enhanced efficient frontier to identify realistic portfolios. It was shown that the architecture could obtain solutions to a high dimensionality portfolio optimization problem. However, the authors noted further experiments were required to prove effectiveness of the proposed architecture. 


\subsection{Analysis}

This section analyzes the characteristics of the aforementioned papers and discuses the findings. Section 3.5 .1 compares the representation of evolutionary and swarm intelligence algorithms for various portfolio optimization problems. Section 3.5 .2 discusses portfolio models, objectives and constraints which appeared in the reviewed literature. Section 3.5.3 presents solution encoding strategies and related constraint handling techniques. Section 3.5.4 provides an overview of portfolio optimization benchmarks. Lastly, Section 3.5.5 describes various performance measures used to evaluate the performance of evolutionary and swarm intelligence algorithms.

\subsubsection{Representation of Algorithms}

A variety of evolutionary and swarm intelligence algorithms have been proposed to solve the portfolio optimization problem. Figure 3.1 shows that the interest in evolutionary and swarm intelligence algorithms as portfolio optimizers has increased over time, specifically 2000-2010. In general, $68.87 \%$ of the surveyed literature made use EAs while $54.72 \%$ used SI algorithms. There exists an overlap of $23.5 \%$ where publications made use of algorithms belonging to both paradigms. Table 3.1 shows that both paradigms are represented similarly across unconstrained and constrained portfolio optimization as a one, two, and three-objective optimization problem.

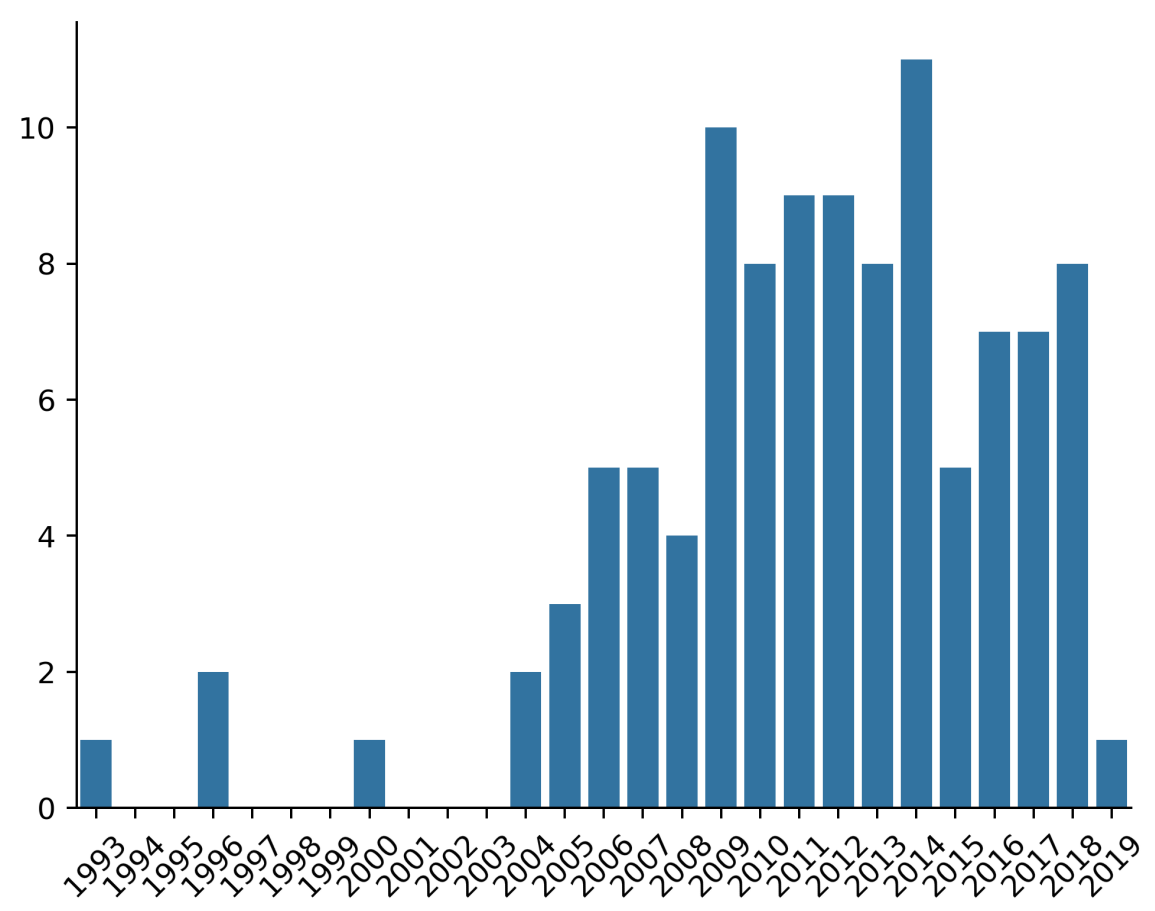

Figure 3.1: Number of publications per year 
Table 3.1: Representation of evolutionary and swarm intelligence paradigms for unconstrained and constrained portfolio optimization

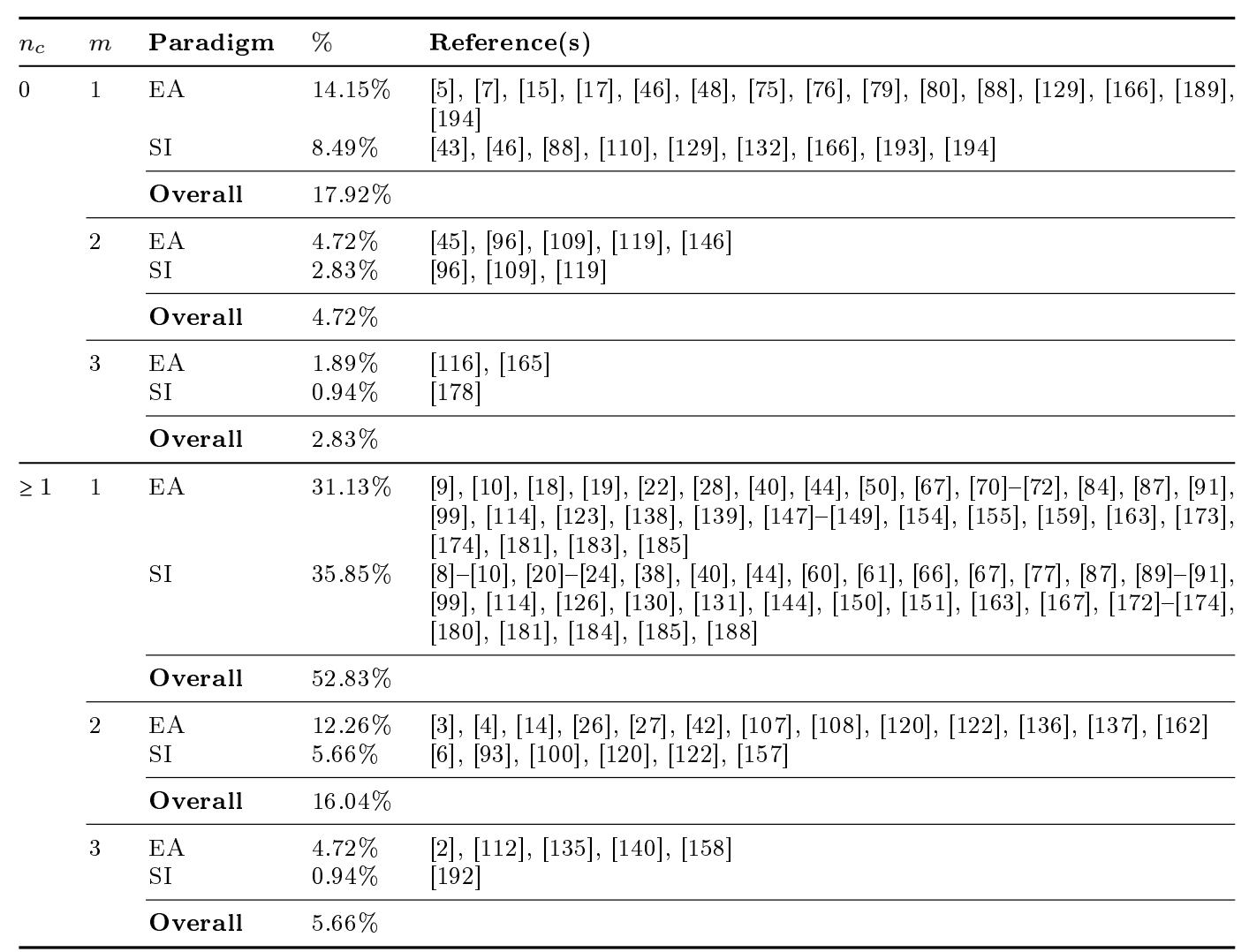

${ }^{*}$ For convenience, $n_{c}$ is the number of constraints and $m$ is the number of objectives.

Single-objective portfolio optimization accounts for $70.75 \%$ of the discussed literature, whereas multi-objective optimization represents $29.25 \%$. Portfolio optimization as a single-objective problem is, thus, overwhelmingly represented. It is crucial to obtain a diverse set of optimal portfolios when making informed investment decisions. Single-objective optimization algorithms converge to a single solution, requiring multiple runs to obtain a set of solutions. In contrast, multi-objective optimization algorithms obtain many solutions, and are thus better suited for portfolio optimization problems.

Table 3.2 shows that GAs are more popular than DE with respect to evolutionary approaches for portfolio optimization. Likewise, PSO is the prominent approach to portfolio optimization amongst SI algorithms, as shown in Table 3.3. GA is, overall, the most popular approach by a margin of $30.19 \%$. However, there is a wider range of SI algorithms than EAs. Furthermore, the first GA was published in the 1950s [63], |64], making it comparatively more well-established than the relatively new SI paradigm, which was first seen in the 1990s [49]. With regards to multi-objective optimization, NSGA-II, PESA, SPEA2 and MOPSO are the most popular approaches and have been shown to obtain diverse non-dominated solutions [2]-[4], [42], [108], 120], 158]. A 
Table 3.2: Evolutionary algorithms for portfolio optimization

\begin{tabular}{|c|c|c|c|c|}
\hline Algorithm & $m$ & $n_{c}$ & $\%$ & Reference(s) \\
\hline \multirow[t]{8}{*}{$\mathrm{DE}$} & \multirow[t]{2}{*}{1} & 2 & $1.89 \%$ & 87,114 \\
\hline & & 3 & $0.94 \%$ & 139 \\
\hline & \multirow[t]{3}{*}{2} & 0 & $1.89 \%$ & 109,146 \\
\hline & & 2 & $0.94 \%$ & 136 \\
\hline & & 3 & $0.94 \%$ & 137 \\
\hline & \multirow[t]{2}{*}{3} & 0 & $0.94 \%$ & 116 \\
\hline & & 2 & $1.89 \%$ & 135,140 \\
\hline & \multicolumn{2}{|l|}{ Overall } & \multicolumn{2}{|l|}{$9.43 \%$} \\
\hline \multirow[t]{16}{*}{ GA } & \multirow[t]{7}{*}{1} & 0 & $14.15 \%$ & 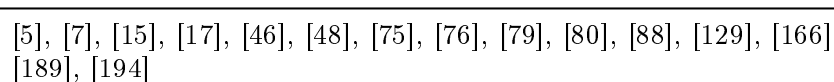 \\
\hline & & 1 & $3.77 \%$ & $28,99,154,155$ \\
\hline & & 2 & $15.09 \%$ & 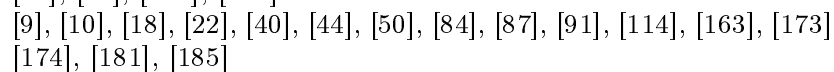 \\
\hline & & 3 & $8.49 \%$ & $19,70,71,[123,139], 147-149,183$ \\
\hline & & 4 & $1.89 \%$ & 72,138 \\
\hline & & 5 & $0.94 \%$ & 67 \\
\hline & & 6 & $0.94 \%$ & 159 \\
\hline & \multirow[t]{4}{*}{2} & 0 & $4.72 \%$ & $45,96,109,119,146$ \\
\hline & & 2 & $7.55 \%$ & $3,[4,42,107,108,120], 122,162$ \\
\hline & & 3 & $0.94 \%$ & 14 \\
\hline & & 4 & $1.89 \%$ & 26,27 \\
\hline & \multirow[t]{4}{*}{3} & 0 & $1.89 \%$ & 116,165 \\
\hline & & 2 & $2.83 \%$ & $2,135,140$ \\
\hline & & 3 & $0.94 \%$ & 112 \\
\hline & & 4 & $0.94 \%$ & 158 \\
\hline & \multicolumn{2}{|l|}{ Overall } & \multicolumn{2}{|l|}{$66.98 \%$} \\
\hline
\end{tabular}

* For convenience, $n_{c}$ is the number of constraints and $m$ is the number of objectives.

limitation of the current research is that the performance of other approaches is largely unexplored to the same extent as GAs and PSO. Investing in these algorithms could be a potentially fruitful area of future work since initial results are promising. Furthermore, the hybridization of these algorithms have been shown to yield superior results over their individual counterparts [93], [126, 172. 
Table 3.3: Swarm intelligence algorithms for portfolio optimization

\begin{tabular}{|c|c|c|c|c|}
\hline Algorithm & $m$ & $n_{c}$ & $\%$ & Reference(s) \\
\hline \multirow[t]{4}{*}{$\mathrm{ABC}$} & 1 & 1 & $0.94 \%$ & 77 \\
\hline & & 2 & $11.32 \%$ & 19, 10, 20, 21], 66, 87, 163, 167, 172, 181 \\
\hline & 2 & 2 & $0.94 \%$ & 100 \\
\hline & Overall & & $13.2 \%$ & \\
\hline \multirow[t]{4}{*}{$\mathrm{ACO}$} & 1 & 0 & $2.83 \%$ & [43, 46, 193 \\
\hline & 2 & 0 & $0.94 \%$ & 96 \\
\hline & & 2 & $0.94 \%$ & 6 \\
\hline & Overall & & $4.72 \%$ & \\
\hline $\mathrm{BA}$ & 1 & 2 & $1.89 \%$ & 89, 163 \\
\hline \multirow[t]{4}{*}{$\mathrm{BFO}$} & 1 & 1 & $0.94 \%$ & 131 \\
\hline & & 2 & $0.94 \%$ & 91 \\
\hline & 2 & 2 & $0.94 \%$ & 122 \\
\hline & Overall & & $2.83 \%$ & \\
\hline $\mathrm{CRO}$ & 1 & 1 & $0.94 \%$ & 184 \\
\hline $\mathrm{CSO}$ & 1 & 2 & $1.89 \%$ & 89,90 \\
\hline FA & 1 & 2 & $6.6 \%$ & $8-10,163,172-174$ \\
\hline FWA & 1 & 2 & $1.89 \%$ & 9 , 163 \\
\hline IWO & 1 & 1 & $0.94 \%$ & 144 \\
\hline $\mathrm{KH}$ & 1 & 2 & $2.83 \%$ & 9 , 163,174 \\
\hline \multirow[t]{11}{*}{$\overline{\mathrm{PSO}}$} & 1 & 0 & $7.55 \%$ & $43,88,110,129,132,166,193,194$ \\
\hline & & 1 & $4.72 \%$ & $\overline{61}, \overline{99}, \overline{131}, \overline{144}, \overline{180}$ \\
\hline & & 2 & $13.21 \%$ & $\left.\left.\left.\left.\frac{22}{185}, 188,40,144,87,89\right], 91\right], 114,126\right], 130,150\right], 181$ \\
\hline & & 3 & $3.77 \%$ & $24,38,60,151$ \\
\hline & & 5 & $0.94 \%$ & 67 \\
\hline & 2 & 0 & $1.89 \%$ & 109,119 \\
\hline & & 2 & $0.94 \%$ & 120 \\
\hline & & 3 & $1.89 \%$ & 93,157 \\
\hline & 3 & 0 & $0.94 \%$ & 178 \\
\hline & & 2 & $0.94 \%$ & 192 \\
\hline & Overall & & $36.79 \%$ & \\
\hline
\end{tabular}

\footnotetext{
${ }^{*}$ For convenience, $n_{c}$ is the number of constraints and $m$ is the number of objectives.
}

Although a variety of algorithms have been proposed for portfolio optimization, it is important to note that if an algorithm excels at one optimization problem, it may not necessarily be suitable for another [182]. Furthermore, deriving algorithms from natural phenomena for the sake of being new and novel may stray research away from scientific rigor $[160]$.

\subsubsection{Portfolio Models}

The mean-variance model is the most widely considered portfolio model for portfolio optimization, represented in the majority of the considered work. Mean-semivariance, considered by $4.72 \%$ of publications [7], [19], [66, [80, 
[108], has been studied considerably less and the remaining proportion of work, that considered alternative portfolios models and $\bar{\sigma}$ measures, even more so. Example models are VaR [108], [116], CVaR [108], [116], prospect theory [110], [129], Sharpe ratio [5], [194], and MAD [19].

Single-objective portfolio optimization related work considered a trade-off between $R$ and $\bar{\sigma}$, as expressed in equation (3.1), as an objective function [9], [18], [60], [66], [89], [174], [184]. Minimization of $\bar{\sigma}$ or maximization of $R$ with a minimum $R$ or maximum $\bar{\sigma}$ constraint, respectively, has also been considered [10], [129], [154], [159], |166].

Optimization of $R$ and $\bar{\sigma}$ are the most widely considered objectives for multi-objective portfolio optimization. Additional objectives, such as liquidity [192], future risk [165], [178], and transaction costs [116] are largely under represented yet vital when considering portfolios. Cardinality, as an additional objective to be minimized, is a novel approach to obtaining a diverse POF of differently size portfolios $[2]$. Similarly, diversity as an objective to be maximized has been shown to guide algorithms to more diverse solutions [158. Table 3.1 shows that only $5.66 \%$ of papers considered portfolio optimization as a three-objective optimization problem.

Portfolio optimization as a fuzzy optimization problem has also been studied [144], 178]. Fuzzy logic is introduced to represent the uncertainty of future returns. Multi-period portfolio models consider the optimization of a portfolio at several time periods 135 . However, this is different from a dynamic problem with a changing search space in the sense that the expected returns for each time period are known up front. Practically, this shows an algorithm's ability to optimize a portfolio subject to rebalancing. Research regarding futures markets, although practically useful, is largely unexplored [136|, |140|. Likewise, there is very little research based on real-world investment strategies, like the 130-30 strategy [135], risk budgeting [135]-[137], and equity market neutral portfolio strategy [137]. Current research has not yet investigated the use of evolutionary and swarm intelligence algorithms as prediction methods for future prices and then making investment decisions based on these predicted prices.

A significant amount of research has treated portfolio optimization as a constrained optimization problem. This is done by adding additional constraints to the considered portfolio model, such as those shown in Table 3.4. The floor and ceiling, and cardinality constraints of equations $(3.6)$ and (3.8), respectively, are the most considered constraints, across all $m$ values, as a result of the popularity of the CCPP. However, Chang et al. noted that single-objective optimization algorithms that optimized the CCPP would effectivity be blind to solutions that could not be obtained by varying $\lambda$. Table 3.5 shows, that in general, publications considered two constraints for constrained portfolio optimization. The popularity of these constraints is attributed to the novel work done by Chang et al. [18]. Considering return as a constraint is particularly useful when trying to find portfolios that would outperform competitors. 
Transaction lots, transaction costs, and sector capitalization have also been studied, albeit to a lesser extent. Such constraints make portfolio optimization more realistic. Other constraints identified, categorized as pre-assignment constraints, are not related to the objective function but rather the search space. Examples of pre-assignment constraints are to remove assets from the search space if they do not meet a given level of risk, return or liquidity [14], [72], [79], [157]. Heavily constrained portfolio optimization problems have been proposed, but are not as popular. Work done by Soleimani et al. [159 considered minimum turnover, asset boundaries, cardinality, sector capitalization, transaction costs, and transaction lots.

Table 3.4: Commonly considered constraints for portfolio optimization

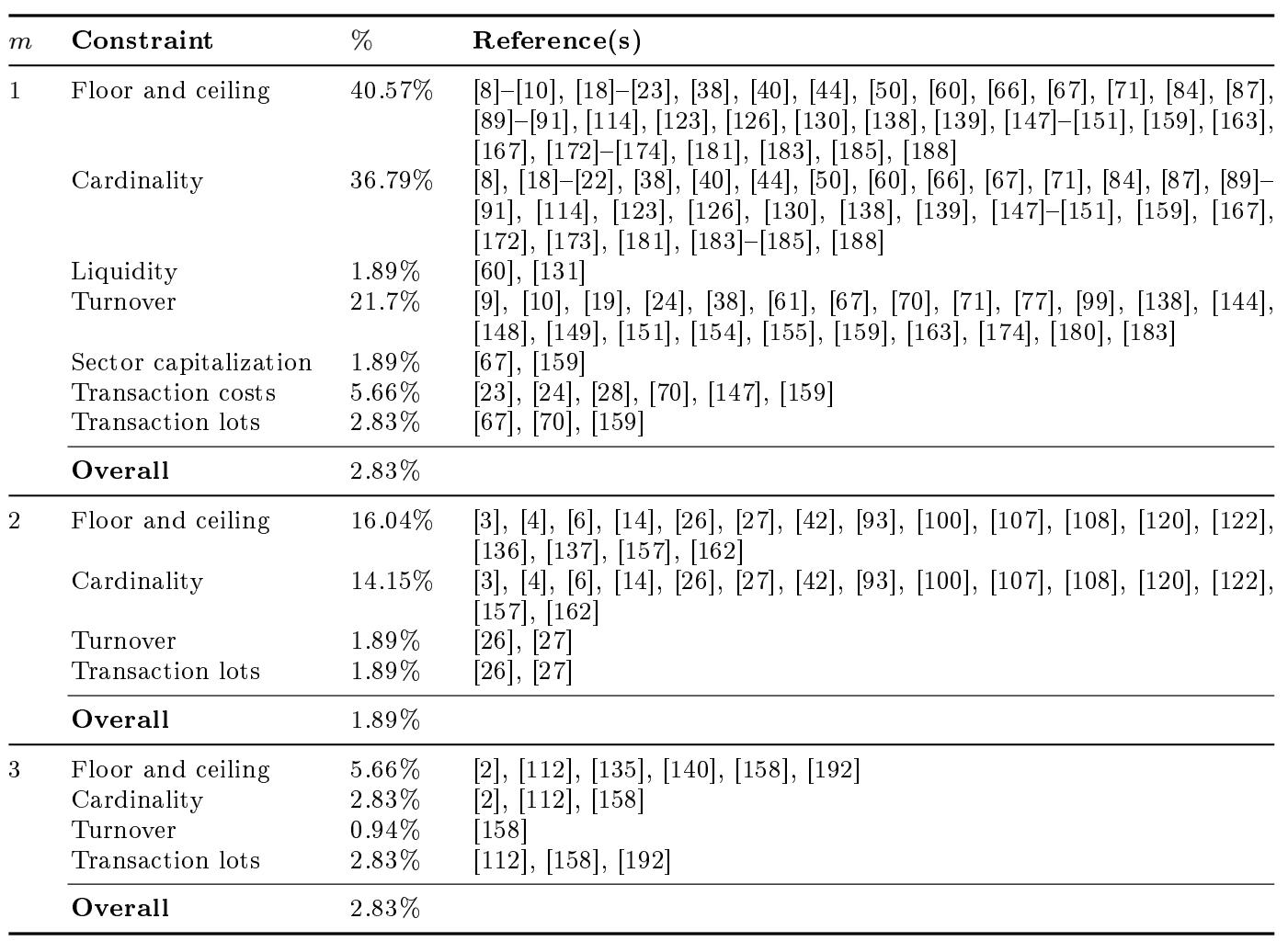

* For convenience, $m$ is the number of objectives. 
Table 3.5: The number of constraints considered for portfolio optimization per number of objectives

\begin{tabular}{|c|c|c|c|}
\hline$m$ & $n_{c}$ & $\%$ & Reference(s) \\
\hline \multirow[t]{2}{*}{1} & $\begin{array}{l}3 \\
4 \\
5 \\
6\end{array}$ & $\begin{array}{l}9.43 \% \\
27.36 \% \\
12.26 \% \\
1.89 \% \\
0.94 \% \\
0.94 \%\end{array}$ & 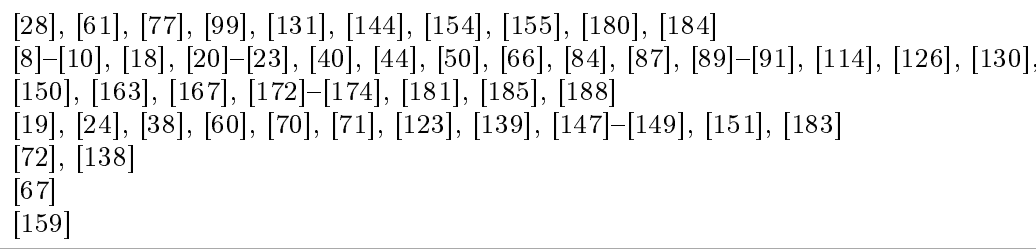 \\
\hline & Overall & $52.83 \%$ & \\
\hline \multirow[t]{2}{*}{2} & $\begin{array}{l}2 \\
3 \\
4\end{array}$ & $\begin{array}{l}10.38 \% \\
3.77 \% \\
1.89 \%\end{array}$ & 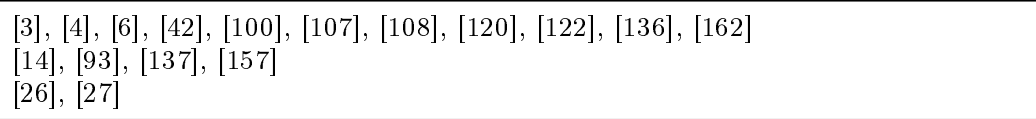 \\
\hline & Overall & $16.04 \%$ & \\
\hline \multirow[t]{2}{*}{3} & $\begin{array}{l}2 \\
4\end{array}$ & $\begin{array}{l}3.77 \% \\
0.94 \%\end{array}$ & \begin{tabular}{|l|l|}
2 & 135 \\
158 & 140, \\
\end{tabular} \\
\hline & Overall & $5.66 \%$ & \\
\hline
\end{tabular}

${ }^{*}$ For convenience, $n_{c}$ is the number of constraints and $m$ is the number of objectives.

\subsubsection{Solution Encoding and Quality}

The encoding scheme used to represent solutions can have a direct impact on the performance of an algorithm [5], [26], [27], [108]. Furthermore, there exists a variety of methods to ensure feasibility of solutions. The most prominent encoding is a direct representation of a portfolio as a collection of asset weights. The popularity of this encoding can largely be attributed to work which considered the unconstrained portfolio problem. However, when constraints are considered and no constraint handling techniques are used, a direct representation frequently leads to infeasible solutions [154]. A simple approach to ensuring that the budget constraint of equation (3.4) is satisfied is to normalize the asset weights.

Popularized by Chang et al. [18], binary decision variables to indicate the inclusion of an asset in the portfolio is commonly used for handling the cardinality constraint. When there are too many or too few assets included, a decision process is then used to remove or add assets, respectively. Such an approach is to randomly select assets to either be included or excluded from the portfolio until the constraint is satisfied [188].

The most commonly used constraint handling technique for the boundary constraint is the free portfolio proportion method, originally presented by Chang et al [18]. Formally, the technique is described as follows:

$$
\begin{aligned}
& F=1-\sum_{i=1}^{n} \varepsilon_{i} \\
& w_{i}=\varepsilon_{i}+\frac{w_{i}}{\sum_{i=1}^{n} w_{i}} F
\end{aligned}
$$


where $F$ is the free remaining proportion of the portfolio, and $w_{i}$ is the portion of asset $i$. So far, this satisfies the lower boundary and budget constraints. An iterative procedure that recalculates the free portion, based on the process above, is performed on portions which violate the upper boundary constraint, if any. Furthermore, by initializing individuals of the initial population within $[\varepsilon, \delta]$, the population is guaranteed to satisfy the boundary constraint.

In a constrained search space, optima may exist separated or surrounded by infeasible regions. Under these circumstances, individuals will be discouraged by the infeasible regions from finding the aforementioned optima. Thus, to allow individuals to fully explore the search space, individuals may be allowed to move through infeasible regions subject to a given tolerance threshold $[8]-[10]$, [87], [163], [172], [174]. Individuals that violate constraints or have exceeded a tolerance threshold can be removed by re-initialization of the individual within the bounds of the search space. A similar but more relaxed approach is to allow individuals to violate constraints. However, such individuals are penalized on how severely the constraints are violated [22], [23], [66], |67], |120], |144|.

$k$-means clustering has been used to group similar performing assets into $k$ groups [138]. The objective function is then optimized by finding optimal combinations of assets from each cluster. This strategy can be used to with the cardinality constraint where the number of groups is equal to the cardinality constraint. However, the quality of solutions found is dependent on the clusters identified. For example, high performing assets could be grouped into a single cluster, thus requiring less desirable assets to be included in the portfolio.

A hybrid encoding of asset weights and indices has been used to represent solutions to the cardinality constrained portfolio optimization problem [15], [20], [21], [60], [159]. This approach works well for problems that consider the cardinality constraint as an equality. By setting the size of a solution equal to $K$, the cardinality constraint is eliminated. Furthermore, the algorithm benefits from computational efficiency as the encoding is invariant to the size of the portfolio problem and the algorithm only needs to evaluate a subset of assets. If the cardinality constraint is treated as an inequality, a third vector of decision variables, representing the inclusion of assets in the portfolio, is introduced [108]. In this encoding strategy, the size of each vector is bound to the cardinality bounds. Thus, the complexity of the cardinality constraint is significantly reduced, while being extremely computationally efficient.

An order-based variation of the asset weights and indices encoding, proposed by Chiam et al. [26], [27], evaluates a solution in a left to right order, similar to that of a bin packing problem. Shoaf and Foster proposed the use of portion indices to represent portfolios [154|. Set-based encodings have also been shown to be effective representations [123], [148], [183]. Lastly, a treebased representation where leaf nodes represented the portion allocated to an asset demonstrated superior performance to that of an asset weights representation for GA $|5|$. 


\subsubsection{Benchmark Problems}

A variety of data sets have been used to benchmark the performance of evolutionary and swarm intelligence algorithms for portfolio optimization. A data set, for example, might contain the expected return and risk for each asset, the correlation value between each asset pair and known optimal solutions.

The OR Library $2^{2}$ was used in approximately $37 \%$ of publications, making it the most widely used data set for portfolio optimization [11], [18]. Summarised in Table 3.6, the OR Library contains five portfolio optimization benchmark problems, each containing real-world data from different stock markets with varying numbers of assets. The benchmark problems were initially published alongside the CCPP by Chang et al. [18]. The benchmark problems test the scalability and accuracy of an algorithm using data from various real world stock markets. Each benchmark problem contains the following: the number of assets, the mean return, the standard deviation of returns for each asset and the correlation value for all possible pairs of assets. The covariance between the returns of assets $i$ and $j$ is calculated as the product of the standard deviation of assets the returns of $i$ and $j$ and the correlation value of $i$ and $j$. A POF containing 2000 pairs of $R$ and $\bar{\sigma}$ values is also provided for each benchmark problem.

Table 3.6: Summary of the OR Library data sets for portfolio optimization

\begin{tabular}{lll}
\hline Stock Market & Region & Number of Assets \\
\hline Hang Seng & Hong Kong & 31 \\
DAX 100 & Germany & 85 \\
FTSE 100 & UK & 89 \\
S\&P 100 & USA & 98 \\
Nikkei 225 & Japan & 225 \\
\hline
\end{tabular}

However, the OR Library is opinionated in the sense that it uses meanvariance to model the expected returns and risk of assets. The majority of publications used independent data sets. In some cases, data was randomly generated to evaluate algorithms' ability to scale to extremely large asset spaces, i.e. more than 1000 assets [46], [50]. It has been shown that algorithms suffer from the curse of dimensionality problem 183. Twenty eight percent of papers considered only one market with an average of approximately 150 assets. Some considered as few as 4, 5, 7, 8 or 10 assets [9], [61], |70], |75], |77], |174]. The remaining portion of independent data sets considered, on average, three markets ranging from 70 to 350 assets. This is a possible flaw in the surveyed literature as different data sets may be easier to optimize than others resulting in a false sense of confidence in the tested algorithms. This makes the cross comparison of algorithms difficult. Furthermore, due to the lack of availability of the data sets used, work cannot successfully be reproduced.

${ }^{2}$ http://people.brunel.ac.uk/mastjjb/jeb/orlib/portinfo.html 


\subsubsection{Performance Measures}

The following performance measures, presented below, have been used to evaluate the usefulness of evolutionary and swarm intelligence algorithms in the context of portfolio optimization. Naturally, statistical measures such as mean, median, minimum, maximum and standard deviation, can be applied to the results of these performance measures, where applicable.

\subsubsection{General Measures}

The simplest performance measure is to compare the fitness scores obtained by the competing algorithms, where the fitness score is defined by the given objective function. However, a more accurate approach is to compare $R$ and $\bar{\sigma}$ values. This indicates whether an algorithm is capable of obtaining superior solutions, than a competing approach. Some benchmark problems include data from a later date to assets how the obtained portfolios would have performed. Being able to obtain good-quality solutions in an efficient manner is also important as investment decisions are often subject to time constraints, particularly in volatile markets. Therefore, the time taken for the algorithm to reach the stopping condition should be minimized. Similarly, the number of evaluations required by each algorithm to obtain a specific or best objective value can also be compared.

\subsubsection{Pareto Optimality Related Measures}

Generational distance (GD) quantifies an algorithm's ability to approximate the Pareto-optimal front from the obtained POF to the true POF [175], 176]. Formally, GD is defined as

$$
G D=\frac{\sqrt{\sum_{i=1}^{|Q|} d_{i}{ }^{2}}}{|Q|}
$$

where $Q$ is the obtained POF and $d_{i}$ is the Euclidean distance between the $i$ 'th solution in the obtained POF and the nearest solution in the true POF, $Q_{\text {true }}$. The GD is to be minimized.

The inverted generational distance (IGD), like GD, measures the closeness of an obtained POF to the true POF, and also the diversity of the obtained POF [37], [171]. IGD, to be minimized, is formulated as

$$
I G D=\frac{\sqrt{\sum_{i=1}^{\left|Q_{\text {true }}\right|} d_{i}^{2}}}{\left|Q_{\text {true }}\right|}
$$

The spacing metric, which measures the diversity of an obtained POF, is defined as

$$
\bar{S}=\sqrt{\frac{1}{|Q|-1} \sum_{i=1}^{|Q|}\left(\bar{d}-d_{i}\right)^{2}}
$$


with

$$
d_{i}=\min _{x_{j} \in Q \wedge j \neq i} \sum_{k=1}^{n}\left|x_{i, k}-x_{j, k}\right|
$$

and

$$
\bar{d}=\sum_{i=1}^{|Q|} \frac{d_{i}}{|Q|}
$$

where $d_{i}$ is the Euclidean distance in objective space between solution $i$ and its nearest neighbor in $\mathrm{Q}$.

The error rate, $E R$, is the proportion of solutions within an obtained POF that are not members of the true POF. This metric, which is to be minimized, is an indicator of how well an algorithm is able to find the true POF. ER is formally defined as

$$
E R=\frac{\sum_{i=1}^{|Q|} e_{i}}{|Q|}
$$

where $e_{i}$ is a binary variable indicating solution $i$ 's absence from the $Q_{\text {true }}$.

The convergence metric, $C$, does not require the use of the true POF. $C$, given two sets of non-dominated solutions $A$ and $B$, indicates the percentage of solutions in $B$ which are dominated by $A$. The performance measure is expressed as

$$
C(A, B)=\frac{|\{b \in B \mid \exists a \in A: a<b\}|}{|B|}
$$

Hypervolume (HV) quantifies the volume of the objective space dominated by an obtained POF bounded by a reference point [195]. HV, to be maximized, is calculated using

$$
H V=\operatorname{volume}\left(\sum_{k=1}^{|Q|} V_{k}\right)
$$

where $V_{k}$ is the hypercube constructed between the reference point and solution $q_{k} \in Q$.

Algorithm effort, $A E$, is the ratio of computational time to the number of solutions in the obtained POF. Formally, AE is expressed as

$$
A E=\frac{T}{|Q|}
$$

where $T$ is the computational time to obtain $Q$.

The spread measure, referred to as $\Delta$, evaluates the diversity of the obtained POF [41]. A larger $\Delta$ value indicates that the approximated Pareto front may not be evenly distributed in the objective function space, and the search may be stuck only in certain regions. The spread measure is defined as

$$
\Delta=\frac{d_{f}+d_{l}+\sum_{k=1}^{|Q|}\left(\bar{d}-d_{i}\right)}{d_{f}+d_{l}+(|Q|-1) \bar{d}}
$$


where $d_{f}$ and $d_{l}$ are the Euclidean distances between the extreme points in the true POF and the extreme points in the obtained POF.

\subsection{Summary}

This chapter conducted a thorough review of the current state of portfolio optimization with respect to evolutionary and swarm intelligence algorithms. The necessary background on portfolio optimization concepts, such as objectives, constraints and portfolio models, was provided. Furthermore, a brief introduction to the types of algorithms examined in this chapter was also given. The discussion and analysis that followed showed that evolutionary and swarm intelligence algorithms are well-established approaches to portfolio optimization. Decades of work have produced a variety of unique and successful approaches to identifying optimal portfolios. Particularly, genetic algorithms (GAs) and particle swarm optimization (PSO) are the most popular approaches. The performance of other algorithms is largely an unexplored area, however, initial results are promising. The majority of work has considered portfolio optimization as a single-objective optimization problem, although, multi-objective approaches have shown to be capable of obtaining higher quality solutions. Constrained portfolio optimization typically included a limit on the number of assets included in the portfolio and boundaries on how much an asset can contribute to the overall portfolio. A variety of other constraints have also been studied, however, to a lesser extent. For large scale portfolio optimization problems, a degradation in performance was generally reported. No work has formulated portfolio optimization as a dynamic optimization problem, which is more indicative of real world daily trading. Furthermore, real world investing is a complex multifaceted problem, and by learning from existing investment strategies, better and more problem-specific algorithms can be designed. 


\section{Chapter 4}

\section{Set-based Particle Swarm Optimization for Portfolio Optimization}

The previous chapter showed that portfolio optimization is rich with interesting and highly competitive algorithms. It also shed light on the fact that existing approaches to portfolio optimization suffer from stability issues [183]: As the number of assets under consideration increases, solution quality deteriorates and the duration of the search is longer. Redefinition of the portfolio optimization problem as a set-based optimization problem has been shown to address the aforementioned scalability issues [124], [148], [183]. A solution to the set-based portfolio optimization problem represents a subset of assets. A set-based solution can then be used to form the search space for a secondary optimization task, where the weighting of each asset is optimized. Set-based algorithms have lead to improved performance in comparison to their non-set based counterparts, although they are dependent on quadratic programming to optimize the asset weights. This chapter 1 presents a new approach to the set-based portfolio optimization problem that uses SBPSO, and unlike previously proposed set-based approaches, a meta-heuristic is used as the weight optimizer for the selected assets.

The remainder of this chapter is organised as follows: Four popular algorithms for portfolio optimization are discussed in Section 4.1. Section 4.2 presents the proposed approach to portfolio optimization and gives an example scenario. The constraint handling technique used by the algorithms is described in Section 4.3. The empirical process used to assess the proposed approach is given in Section 4.4. Section 4.5 presents and discusses the results.

\footnotetext{
${ }^{1}$ This chapter was published in the proceedings of the ANTS conference; and in lecture notes in computer science under the title "Set-based particle swarm optimization for portfolio optimization" |58. This chapter is also currently being reviewed for the journal Applied Soft Computing under the title "Set-based particle swarm optimization for portfolio optimization: Empirical analysis" 102 .
} 
The findings are summarised in Section 4.6 .

\subsection{Algorithms for Portfolio Optimization}

This section discusses four state-of-the-art algorithms (identified in the previous chapter) for the mean-variance portfolio optimization problem of equation (3.1). The algorithms, ABC, FA, GA and PSO, were selected based on their popularity and competitiveness, and are used later in this Chapter to compare the performance of the proposed SBPSO approach. Sections 4.1.1, 4.1.2, 4.1 .3 and 4.1.4 present ABC, FA, GA and PSO for portfolio optimization, respectively.

\subsubsection{Artificial Bee Colony for Portfolio Optimization}

$\mathrm{ABC}$ is a highly competitive and popular algorithm for portfolio optimization. This section outlines the original ABC algorithm which was first used by Chen et al. to optimize the mean-variance model of equation (3.1) [20]. The colony consists of three types of bees: employed bees, onlooker bees and scout bees. The bees are attracted to food sources, which are randomly initialized throughout the search space. The number of employed bees, onlooker bees and scout bees are equal to the number of food sources. Each employed bee moves towards a food source, $\boldsymbol{x}_{i}$, and determines a neighboring food source, $\boldsymbol{o}_{i}$, using

$$
\boldsymbol{o}_{i}=\boldsymbol{x}_{i}+\boldsymbol{\varphi}_{i}\left(\boldsymbol{x}_{i}-\boldsymbol{x}_{k}\right)
$$

where $\boldsymbol{\varphi}_{i}$ is a vector of uniform random values in the range $[-1,1]$, and $\boldsymbol{x}_{k}$ is a randomly chosen food source. Then, the employed bee selects the most abundant food source between $\boldsymbol{x}_{i}$ and $\boldsymbol{o}_{i}$, and dances. In the case of maximization problems, the amount of nectar available at each food source is determined by the objective function value. However, in the case of minimization problems, e.g. the mean-variance portfolio optimization problem, the nectar of $\boldsymbol{x}_{i}$ is determined by

$$
\kappa_{i}= \begin{cases}\frac{1}{1+f\left(\boldsymbol{x}_{i}\right)} & \text { if } f\left(\boldsymbol{x}_{i}\right) \geq 0 \\ 1+\left|f\left(\boldsymbol{x}_{i}\right)\right| & \text { if } f\left(\boldsymbol{x}_{i}\right)<0\end{cases}
$$

where $\kappa_{i}$ is the nectar of food source $i$, and $f$ is the objective function.

Next, each onlooker bee chooses a food source based on a probability determined by the food source's nectar relative to all other food sources. The probability, $p_{i}$, of selecting food source $\boldsymbol{x}_{i}$ is

$$
p_{i}=\frac{\kappa_{i}}{\sum_{j=1}^{n} \kappa_{j}}
$$

where $n$ is the number of food sources. The onlooker bees exploit the selected food source by visiting $\boldsymbol{o}_{i}$. 
If a food source is not updated with a better solution over a fixed number of iterations, scout bees are dispatched to explore new food sources. The number of iterations is determined by the limit control parameter. If the new food source is better than the old food source, it replaces the old food source. The new food source is a randomly generated position within the bounds of the search space. Algorithm 5 contains pseudo code for ABC.

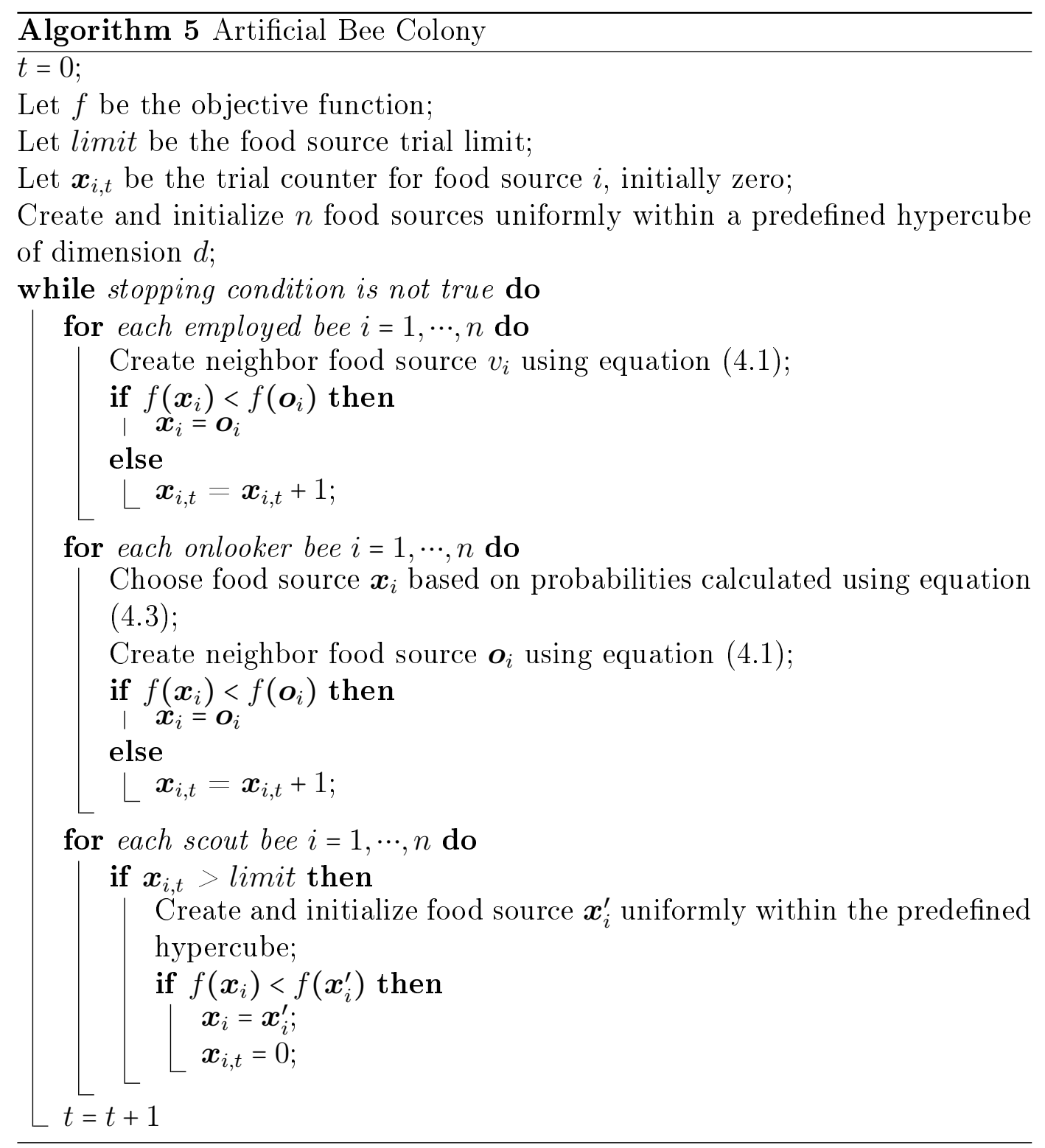

\subsubsection{Firefly Algorithm}

Much of the work centered around FAs for portfolio optimization considered portfolio optimization as a constrained optimization problem [8], [173]. Since this thesis regards portfolio optimization as an unconstrained optimization 
problem, the FA is presented without any of the previously proposed modifications to handle constraints.

Fireflies generate flashes of light by a process of bioluminescence to facilitate communication. Algorithmically, the light intensity of a firefly is implemented as the objective function value. Like $\mathrm{ABC}$, a transformation function can be used to accommodate minimization problems. That transformation function is

$$
I\left(x_{i}\right)= \begin{cases}\frac{1}{1+f\left(\boldsymbol{x}_{i}\right)} & \text { if } f\left(\boldsymbol{x}_{i}\right) \geq 0 \\ 1+\left|f\left(\boldsymbol{x}_{i}\right)\right| & \text { if } f\left(\boldsymbol{x}_{i}\right)<0\end{cases}
$$

where $I$ is the light intensity of firefly $\boldsymbol{x}_{i}$ and $f$ is the objective function.

The attractiveness, $\beta$, of a firefly is relative to the distance between it and the firefly looking at it. The attractiveness between firefly $i$ and firefly $j$ is calculated as

$$
\beta(r)_{i j}=\beta_{0} e^{-\gamma r_{i, j}^{2}}
$$

where $r$ is the Euclidean distance between firefly $i$ and firefly $j, \beta_{0}$ is the attractiveness when $r=0$ and $\gamma$ is the light absorption coefficient that models light absorbed by the air. The distance between firefly $i$ and firefly $j$ is calculated as

$$
r_{i j}=\sqrt{\sum_{k=1}^{d}\left(x_{i, k}-x_{j, k}\right)}
$$

where $d$ is the number of dimensions.

A firefly moves toward another brighter firefly if the move would increase the brightness of the firefly; otherwise, the firefly stays where it is. A firefly updates its position using

$$
\boldsymbol{x}_{i}(t+1)=\boldsymbol{x}_{i}(t)+\beta(r)_{i j}\left(\boldsymbol{x}_{j}-\boldsymbol{x}_{i}\right)+\alpha(g-0.5)
$$

where $\alpha$ controls the influence of $g$, a random number sampled from a uniform distribution in [0,1]. Algorithm 6 presents the FA for portfolio optimization. 


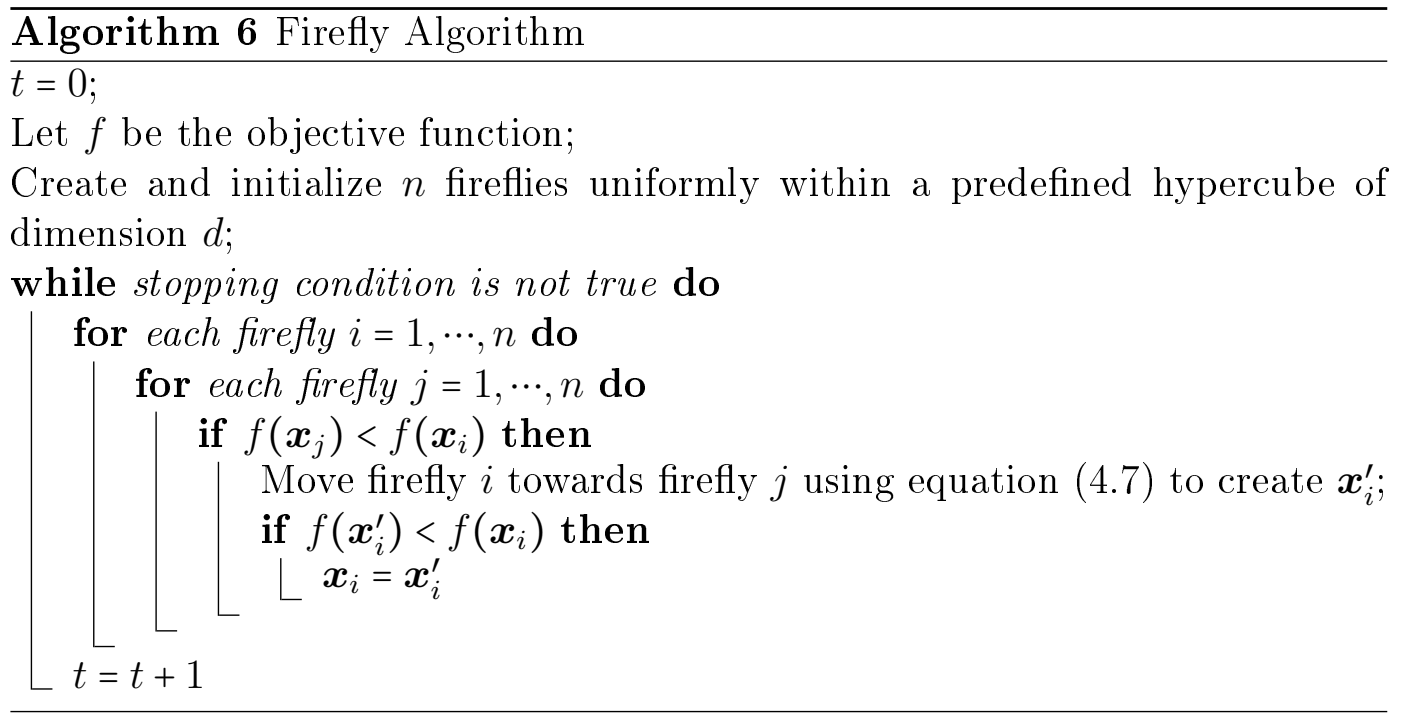

\subsubsection{Genetic Algorithm}

One of the most popular GAs for portfolio optimization was that proposed by Chang et al [18]. The GA which was used to solve the mean-variance portfolio optimization problem is summarised in Algorithm 7. Each individual represents a candidate solution to the mean-variance portfolio optimization problem. The genes of an individual represent the asset weights. The following operators are used to evolve the candidate solutions to the portfolio optimization problem: Tournament selection is used to select parents that recombine to create new individuals. The breeding process, referred to as uniform crossover, selects genes from two parents, where each gene from either parent has an equal chance of being selected. The result of the breeding process is a new individual, referred to as an offspring. Lastly, there is a chance that a randomly chosen gene of the offspring is mutated. The severity of the mutation is determined by the control parameter, $S_{m}$.

\subsubsection{Particle Swarm Optimization}

PSO, specifically inertia-weighted gbest PSO (refer to Section 2.2), has been widely studied as an approach to portfolio optimization [12], [24], [38], [44], [67], [130], [132], [150], [151], [166], [193]. PSO is selected as an algorithm to compare SBPSO because of its popularity amongst the reviewed literature and its ability to obtain good-quality solutions. Algorithm 1 describes PSO, however the velocities are updated using equation 2.5 


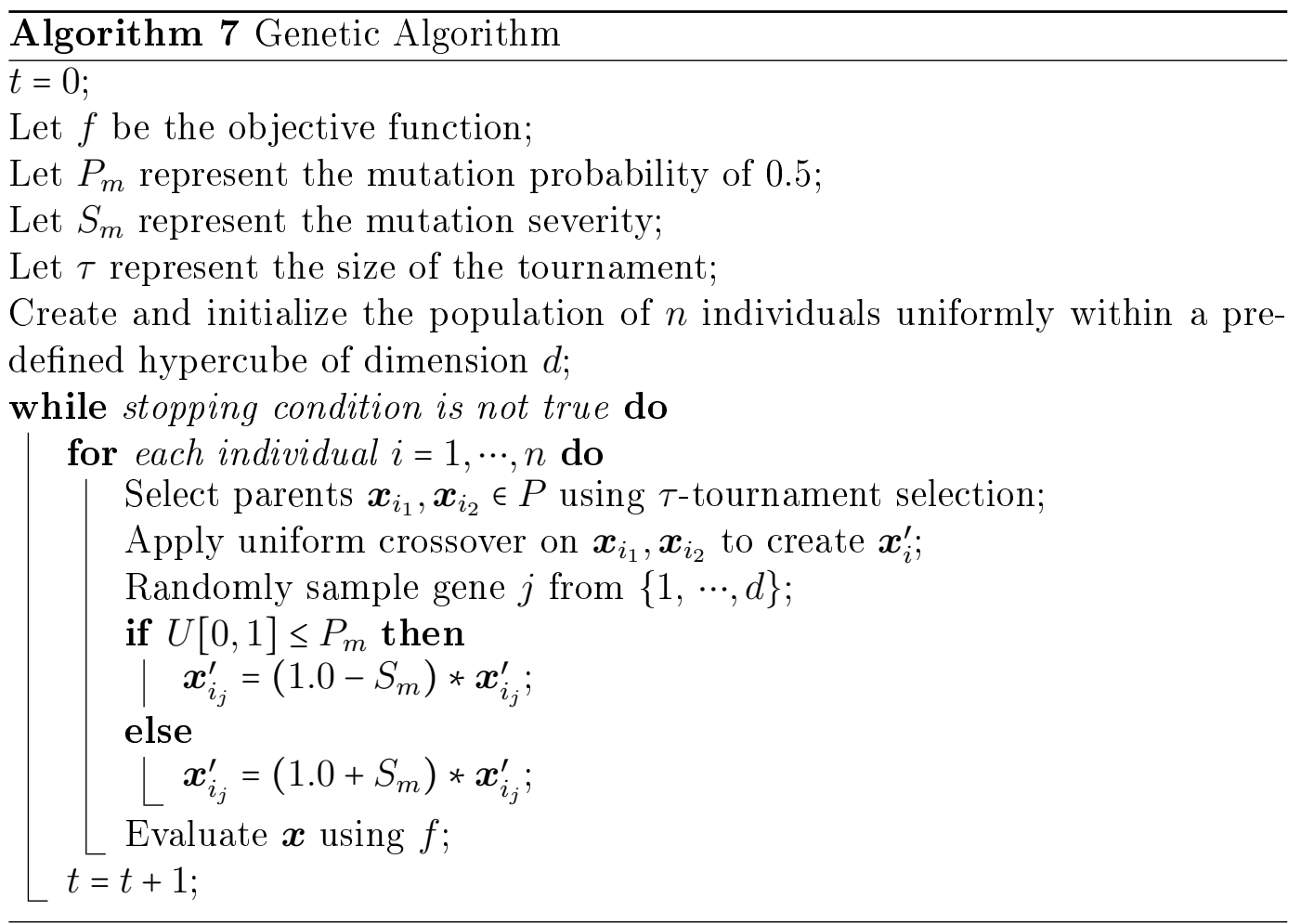

\subsection{A New Approach to Set-based Portfolio Optimization}

The universe for the SBPSO is the set of all assets. The objective of the SBPSO is to select a subset of assets that form the optimal portfolio for the mean-variance portfolio model of equation (3.1). Each particle represents a candidate portfolio of sets, which are randomly initialized. For each of these particles, a secondary optimization problem is solved, i.e to find optimal values for the weights of the assets that minimize the mean-variance portfolio model such that the total budget constraint of equation (3.4) and the non-negative weight constraint of equation (3.5) are satisfied. Algorithm 8 presents the pseudo code for SBPSO for portfolio optimization. 
Algorithm 8 Set-based Particle Swarm Optimization for Portfolio Optimization

$t=0$;

Let $f$ be the function the described in Algorithm 9 ;

Create and initialize a swarm, $S$, of $n$ particles uniformly within a predefined set universe $U$;

for each particle $i=1, \cdots, n$ do

Let $Y_{i}$ represent the personal best position of particle $X_{i}$, initialized to $X_{i}(t)$

Let $\hat{Y}_{i}$ represent the global best position, initialized to the best $X_{i}$;

Initialize $V_{i}(t)$ to \{\} , i.e the empty set;

while stopping condition is not true do

for each particle $i=1, \cdots, n$ do

$$
\text { if } \begin{aligned}
f\left(X_{i}(t)\right) & <f\left(Y_{i}(t)\right) \text { then } \\
\left\llcorner Y_{i}(t+1)\right. & =X_{i}(t) ;
\end{aligned}
$$

Update $\hat{Y}_{i}(t+1)$ to the best $X_{i}(t)$;

for each particle $i=1, \cdots, n$ do

Update particle $i$ 's velocity using equation (2.17);

Update particle $i$ 's position using equation (2.18);

$t=t+1$

The weights represent the contribution of each asset to the portfolio. In other words, the weights represent how much of the total budget should be allocated to purchasing the corresponding asset. An inertia-weighted gbest PSO (refer to Section 2.2) is used as the weight optimizer. Thus, the search space of the inertia-weighted gbest PSO is determined by the assets of the corresponding set-particle. Furthermore, the best objective function value at the end of PSO search is then assigned to the corresponding set-particle. Algorithm 9 summarizes the weight determination stage.

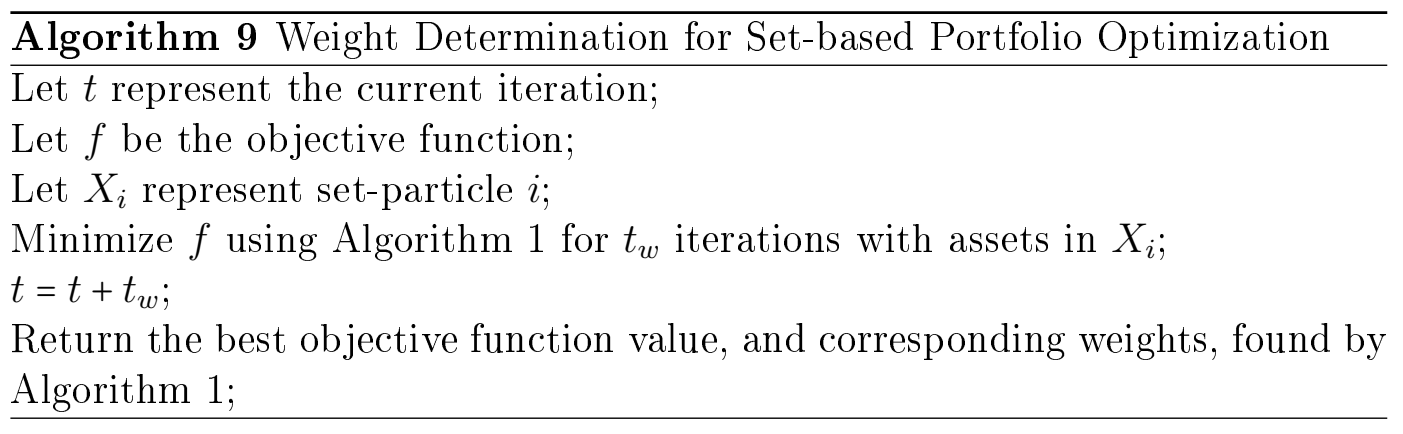

To better illustrate the proposed approach, consider the following example. There are 50 assets in the universe. Initially, a particle randomly selects a subset of assets, say $\{5,12,23,26,31\}$ from the universe. These assets are then 
used to create a continuous search space for the inner PSO. Each dimension in the search space of the PSO represents the weight of an asset. Then the PSO optimizes the asset weights for a fixed duration. The table below illustrates example results obtained by the weight optimizer.

\begin{tabular}{l|c|c|c|c|c}
\hline Assets & 5 & 12 & 23 & 26 & 31 \\
\hline Weights & 0.31 & 0.16 & 0.11 & 0.05 & 0.37 \\
\hline
\end{tabular}

The combination of the assets and weightings is a candidate portfolio. Continuing with the example, the resulting portfolio is visualised below.

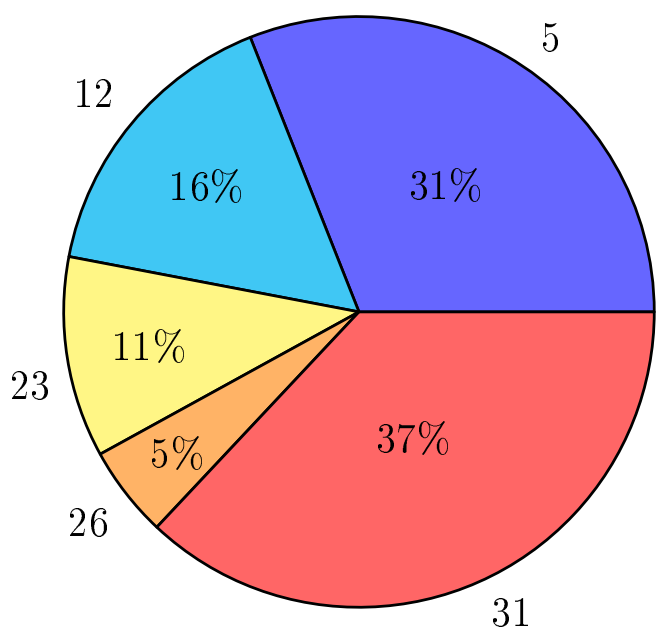

Lastly, the $\odot^{+}$operator for SBPSO is re-implemented as the random selection of $N_{\beta, A}$ elements from $A$ and not using tournament selection. The purpose of this is twofold: $\odot^{+}$implemented using random selection reduces computational complexity of the proposed approach since it would require a weight optimization process fort each candidate in the tournament; and removes the selection bias of the tournament selection. The effects of re-implementing the $\odot^{+}$operator is further studied in Chapter 6 and it is shown that there is no effect on performance.

The proposed SBPSO is expected to perform similarly to the other algorithms for portfolio optimization mentioned in Section 4.1, because the SBPSO uses the inertia gbest PSO as the weight optimizer. Furthermore, the SBPSO reduces the complexity of portfolio optimization by optimizing over subsets of the search space at a time. In comparison, the other algorithms optimize the entire search. Thus, the SBPSO should find better quality solutions for larger portfolio optimization problems than the other algorithms. 


\subsection{Constraint Handling}

This section details the constraint handling techniques used by the algorithms in this chapter. In the case of the SBPSO, the constraint handling techniques are applied in the asset weight optimizer PSO. A position is transformed such that any negative number is treated as zero, satisfying equation (3.5). Then, the position is normalised by dividing each weight in the position by the sum of weights to ensure that the constraint of equation $(3.4)$ is met. Thus, the position now represents the percentages of the total value to be invested for each asset. For example, the position $(2.34,-3.12,0.95,1.84,5.33)$ violates both constraints. Using the described constraint handling technique, the position then becomes $(0.22,0.0,0.09,0.18,0.51)$. For the case that all asset weights are assigned a weight of zero, a sufficiently large objective function value is given to deter the algorithm from that area of the search space.

\subsection{Empirical Process}

This section describes the empirical process used to assess the performance of the proposed SBPSO approach and the other algorithms mentioned in Section 4.1. Note that the same empirical process is followed in subsequent chapters. Section 4.4.1 describes the implementation of the algorithms. The benchmark problems are discussed in Section 4.4.2. Section 4.4.3 describes the parameter tuning process used to optimize the control parameters of each algorithm for portfolio optimization. The performance measures used are listed in Section 4.4.4.

\subsubsection{Implementation of Algorithms}

The algorithms were implemented using the Computational Intelligence library 2 a highly concurrent functional programming library with composable algorithmic abstractions.

\subsubsection{Benchmark Problems}

The OR Library ${ }^{3}$ benchmark problems, summarised in Table 3.6, were used to evaluate the ability of the algorithms to optimize the mean-variance model of equation (3.1) for 50 evenly spaced $\lambda$ values in the range $[0,1]$. The resulting objective function is optimized (minimized) by finding the asset weights that strike the best balance between risk and return. For each $\lambda$ value, the algorithms were given 5000 iterations to optimise the mean-variance model. For the proposed SBPSO approach, SBPSO and the weight optimizer PSO

\footnotetext{
${ }^{2}$ https://github.com/ciren/cilib

${ }^{3}$ http://people.brunel.ac.uk/mastjjb/jeb/orlib/portinfo.html
} 
interleaved every 10 iterations. Furthermore, each algorithm had a population size of 20 , or in the case of the ABC, 20 food sources.

\subsubsection{Control Parameter Tuning}

The control parameters of each algorithm were optimized for each benchmark problem so that the algorithms could be compared fairly. To do so, parameter sets were generated using sequences of Sobol pseudo-random numbers that spanned the parameter space each of each algorithm [62]. The parameter space for each algorithm is summarised in Table 4.1. The parameter ranges were taken from the reviewed literature [18], |20|, [52], [104], [173]. For each algorithm, 128 parameters sets were evaluated. However, in the case of the PSO, only the parameter sets (of the 128 parameters sets) that satisfied the stability conditions of equation (2.6) were considered. The control parameters for the weight optimizer PSO for the SBPSO were not tuned since the portfolio problems it solves vary due to the changes in the positions of set-particles. Thus, recommended parameters for the weight optimizer PSO that satisfy stability conditions and guarantee that an equilibrium state will be reached were used. These are $w=0.729844$ and $c_{1}=c_{2}=1.496180$ [32]. The parameter sets were then ranked according to their average objective function value for each of the $\lambda$ values over 30 independent runs. The parameter set with the best ranking was selected as the optimal control parameter values for each algorithm, and for each benchmark problem. The optimal control parameter values are given in Appendix $\mathrm{D}$.

Table 4.1: Parameter ranges used in the tuning process

\begin{tabular}{lll}
\hline Algorithm & Parameter & Range \\
\hline ABC & limit & {$[0,100]$} \\
\hline FA & $\beta_{0}$ & {$[0.00,1.00]$} \\
& $\gamma$ & {$[0.00,10.00]$} \\
& $\alpha$ & {$[0.00,1.00]$} \\
\hline GA & $S_{m}$ & {$[0.01,0.50]$} \\
& $\tau$ & {$[2,20]$} \\
\hline PSO & $w$ & {$[0.00,1.00]$} \\
& $c_{1}$ & {$[0.00,2.00]$} \\
& $c_{2}$ & {$[0.00,2.00]$} \\
\hline SBPSO & $c_{1}$ & {$[0.00,1.00]$} \\
& $c_{2}$ & {$[0.00,1.00]$} \\
& $c_{3}$ & {$[0.50,5.00]$} \\
& $c_{4}$ & {$[0.50,5.00]$} \\
\hline
\end{tabular}




\subsubsection{Performance Measures}

The following performance measures were used to assess the quality, and efficiency of the obtained solutions: The number of optimal solutions $(\mathrm{N})$, the cardinality/size of the solutions $(K)$, return $(R)$, risk $(\bar{\sigma})$, GD, IGD, HV (refer to Section 3.5.5 and the time in seconds to reach the stopping condition. Time is a useful performance measure since some investment decisions need to happen quickly and frequently. The results were recorded after each iteration and averaged over the 30 independent runs. The size of the solutions was measured as the number of non-zero weighted assets. Furthermore, the best objective function value at the end of every search process for a given $\lambda$ was considered the best solution, and the corresponding return and risk values formed a solution on the obtained POF. Thus, each independent run of the $\lambda$ values resulted in a POF. The mean and standard deviation for each performance measure was recorded and tabulated. Furthermore, to test for any meaningful statistical difference between two algorithms, one-tailed Mann Whitney U tests with a level of significance of $95 \%$ were used. The results of the statistical significance tests were used to rank the algorithms. All performance measures were equally weighted in the ranking process.

\subsection{Results}

This section discusses the results of each algorithm for each benchmark problem. The results are also given in the tables and figures below. Section 4.5.1 presents the Hang Seng results. The DAX 100 results are given in Section 4.5.2. Section 4.5.3 discusses the FTSE 100 results. Section 4.5.4 examines the S\&P 100 results, and the Nikkei 225 results are discussed in Section 4.5.5.

\subsubsection{Hang Seng}

Table 4.2 shows that, on average, ABC obtained higher return values than all other algorithms. The rankings of the statistical significance tests in Table 4.3 confirm that $\mathrm{ABC}$ was the best performing algorithm with regard to return. However, the profitability of $\mathrm{ABC}$ was at the cost of more risk, with $\mathrm{ABC}$ ranking last with respect to risk. On the other hand, FA obtained the worst return value, but the best value for risk. The GA, PSO and SBPSO ranked similarly to each other with regard to return and risk, and their standard deviations for return and risk were significantly smaller than those of the ABC and FA - indicating that the GA, PSO and SBPSO were more consistent in their performance. The similarity in performance of the GA, PSO and SBPSO can also be seen in the size of the portfolios they obtained. On average all three algorithms obtained portfolios that contained three assets. Conversely, the portfolios obtained by $\mathrm{ABC}$ were much larger with an average of 15 assets 
CHAPTER 4. SET-BASED PARTICLE SWARM OPTIMIZATION FOR PORTFOLIO OPTIMIZATION

per portfolio. With regard to the number of optimal portfolios obtained, ABC and FA obtained slightly more solutions than the other algorithms.

Table 4.2: Hang Seng results for each performance measure

\begin{tabular}{llcccccccc}
\hline Algorithm & Result & $\mathbf{N}$ & $K$ & $R$ & $\bar{\sigma}$ & GD & IGD & HV & Time \\
\hline ABC & $\bar{x}$ & 37 & 14 & 0.007848 & 0.002110 & 0.000176 & 0.000210 & 0.814630 & 11.04 \\
& $\sigma$ & 2 & 2 & 0.000142 & 0.000125 & 0.000009 & 0.000000 & 0.000000 & 0.41 \\
\hline FA & $\bar{x}$ & 38 & 5 & 0.006084 & 0.001783 & 0.003959 & 0.000556 & 0.748081 & 38.95 \\
& $\sigma$ & 4 & 1 & 0.000592 & 0.000268 & 0.000485 & 0.000085 & 0.028972 & 3.71 \\
\hline GA & $\bar{x}$ & 32 & 3 & 0.007576 & 0.001884 & 0.000279 & 0.000222 & 0.779399 & 18.10 \\
& $\sigma$ & 1 & 0 & 0.000052 & 0.000042 & 0.000065 & 0.000005 & 0.025320 & 0.64 \\
\hline PSO & $\bar{x}$ & 33 & 3 & 0.007607 & 0.001897 & 0.000219 & 0.000212 & 0.786254 & 15.02 \\
& $\sigma$ & 1 & 0 & 0.000003 & 0.000001 & 0.000049 & 0.000002 & 0.026135 & 0.69 \\
\hline SBPSO & $\bar{x}$ & 34 & 3 & 0.007614 & 0.001908 & 0.000463 & 0.000236 & 0.776926 & 9.37 \\
& $\sigma$ & 1 & 0 & 0.000022 & 0.000009 & 0.000141 & 0.000010 & 0.023747 & 0.53 \\
\hline
\end{tabular}

Table 4.3: Hang Seng rankings for each performance measure

\begin{tabular}{|c|c|c|c|c|c|c|c|c|c|c|}
\hline Algorithm & Result & $\mathbf{N}$ & $K$ & $R$ & $\bar{\sigma}$ & GD & IGD & HV & Time & Overall \\
\hline \multirow[t]{5}{*}{$\mathrm{ABC}$} & Wins & 3 & 0 & 4 & 0 & 4 & 4 & 4 & 3 & 22 \\
\hline & Losses & 0 & 4 & 0 & 4 & 0 & 0 & 0 & 1 & 9 \\
\hline & Draws & 1 & 0 & 0 & 0 & 0 & 0 & 0 & 0 & 1 \\
\hline & Difference & 3 & -4 & 4 & -4 & 4 & 4 & 4 & 2 & 13 \\
\hline & Rank & 1 & 5 & 1 & 5 & 1 & 1 & 1 & 2 & 1 \\
\hline \multirow[t]{5}{*}{ FA } & Wins & 3 & 1 & 0 & 3 & 0 & 0 & 0 & 0 & 7 \\
\hline & Losses & 0 & 3 & 4 & 0 & 4 & 4 & 4 & 4 & 23 \\
\hline & Draws & 1 & 0 & 0 & 1 & 0 & 0 & 0 & 0 & 2 \\
\hline & Difference & 3 & -2 & -4 & 3 & -4 & -4 & -4 & -4 & -16 \\
\hline & Rank & 1 & 4 & 4 & 1 & 5 & 5 & 5 & 5 & 5 \\
\hline \multirow[t]{5}{*}{ GA } & Wins & 0 & 2 & 1 & 2 & 2 & 2 & 1 & 1 & 11 \\
\hline & Losses & 4 & 2 & 3 & 0 & 2 & 2 & 2 & 3 & 18 \\
\hline & Draws & 0 & 0 & 0 & 2 & 0 & 0 & 1 & 0 & 3 \\
\hline & Difference & -4 & 0 & -2 & 2 & 0 & 0 & -1 & -2 & -7 \\
\hline & Rank & 3 & 3 & 3 & 2 & 3 & 3 & 4 & 4 & 4 \\
\hline \multirow[t]{5}{*}{ PSO } & Wins & 1 & 3 & 2 & 2 & 3 & 3 & 2 & 2 & 18 \\
\hline & Losses & 2 & 1 & 1 & 1 & 1 & 1 & 1 & 2 & 10 \\
\hline & Draws & 1 & 0 & 1 & 1 & 0 & 0 & 1 & 0 & 4 \\
\hline & Difference & -1 & 2 & 1 & 1 & 2 & 2 & 1 & 0 & 8 \\
\hline & Rank & 2 & 2 & 2 & 3 & 2 & 2 & 2 & 3 & 2 \\
\hline \multirow[t]{5}{*}{ SBPSO } & Wins & 1 & 4 & 2 & 1 & 1 & 1 & 1 & 4 & 15 \\
\hline & Losses & 2 & 0 & 1 & 3 & 3 & 3 & 1 & 0 & 13 \\
\hline & Draws & 1 & 0 & 1 & 0 & 0 & 0 & 2 & 0 & 4 \\
\hline & Difference & -1 & 4 & 1 & -2 & -2 & -2 & 0 & 4 & 2 \\
\hline & Rank & 2 & 1 & 2 & 4 & 4 & 4 & 3 & 1 & 3 \\
\hline
\end{tabular}

Figure 4.1 shows that all algorithms were able to approximate the POF, except FA. The GD, IGD and HV rankings in Table 4.3 provide further evidence that FA was unable to approximate the POF as it ranked last in all three measures. ABC performed the best out of all the algorithms with regard 
to GD, IGD and HV. Furthermore, the standard deviations of ABC for the aforementioned measures were the lowest. The PSO, which obtained similar results to the ABC, ranked second for GD, IGD and HV. The GA and SBPSO significantly outperformed the FA for the same performance measures, but performed worse than the PSO.

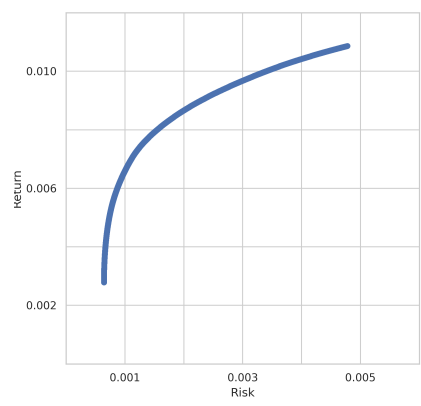

(a) True POF

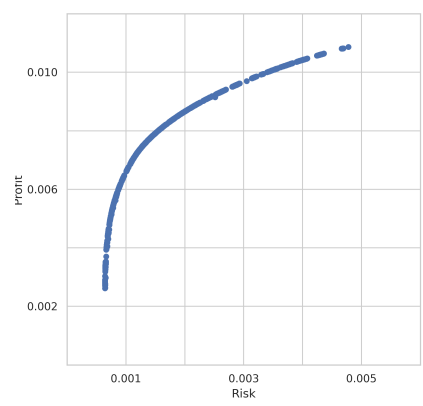

(d) GA

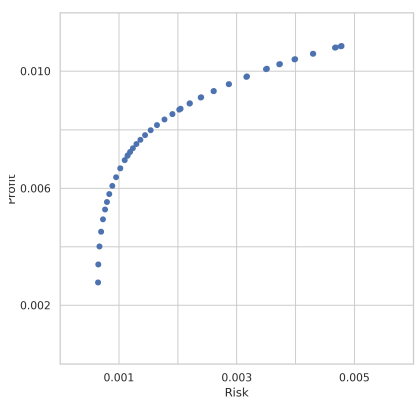

(b) $\mathrm{ABC}$

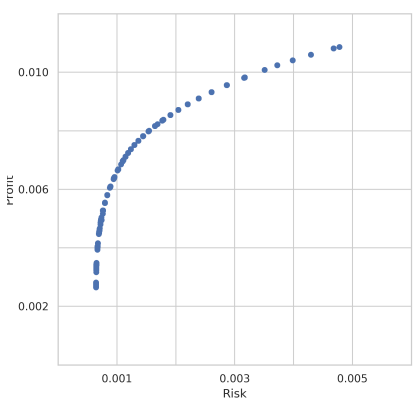

(e) PSO

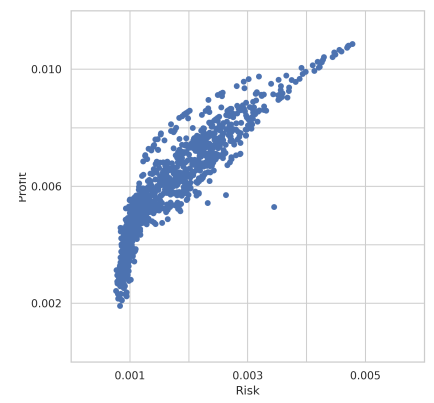

(c) FA

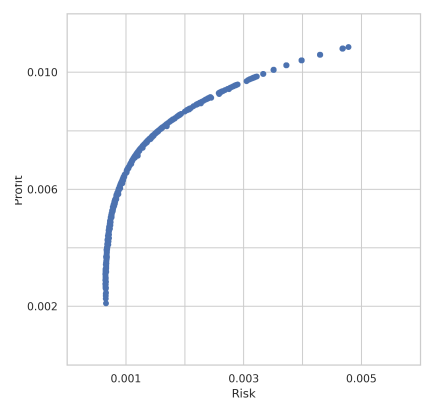

(f) SBPSO

Figure 4.1: Obtained Pareto-optimal fronts for Hang Seng

SBPSO was statistically significantly faster than all the other algorithms in reaching the stopping condition of 5000 iterations, as shown in Table 4.3. SBPSO was approximately four times faster than FA, the slowest algorithm. The second fastest algorithm was ABC, about two seconds slower than SBPSO. The PSO and GA were also quick to reach the stopping condition, despite ranking third and fourth, respectively.

The ABC was the highest ranked algorithm for the Hang Seng benchmark problem, overall. The PSO ranked second and the newly proposed SBPSO ranked third. The lowest ranking algorithms overall were the GA and FA, respectively.

\subsubsection{DAX 100}

Like the previous results, ABC ranked first for return but last for risk, and the FA ranked last in return but first for risk (refer to Table 4.5). Table 4.4 shows 
CHAPTER 4. SET-BASED PARTICLE SWARM OPTIMIZATION FOR PORTFOLIO OPTIMIZATION

that the profitability of the portfolios obtained by $\mathrm{ABC}$ were on average twice that of the FA. Conversely, the average risk value for the FA was almost half that of ABC. The PSO and GA performed similarly with regard to return and risk. The SBPSO, however, underperformed in return.

Table 4.4: DAX 100 results for each performance measure

\begin{tabular}{lccccccccc}
\hline Algorithm & Result & $\mathbf{N}$ & $K$ & $R$ & $\bar{\sigma}$ & GD & IGD & HV & Time \\
\hline ABC & $\bar{x}$ & 49 & 14 & 0.008633 & 0.001219 & 0.000228 & 0.000304 & 0.787923 & 43.68 \\
& $\sigma$ & 1 & 1 & 0.000021 & 0.000029 & 0.000033 & 0.000000 & 0.000000 & 0.56 \\
\hline FA & $\bar{x}$ & 34 & 11 & 0.004485 & 0.000768 & 0.003702 & 0.000568 & 0.844798 & 166.26 \\
& $\sigma$ & 7 & 2 & 0.000606 & 0.000143 & 0.000571 & 0.000103 & 0.033878 & 21.28 \\
\hline GA & $\bar{x}$ & 39 & 10 & 0.008387 & 0.000953 & 0.000295 & 0.000307 & 0.893540 & 50.86 \\
& $\sigma$ & 1 & 0 & 0.000034 & 0.000025 & 0.000152 & 0.000004 & 0.057716 & 2.02 \\
\hline PSO & $\bar{x}$ & 42 & 5 & 0.008456 & 0.000967 & 0.000222 & 0.000304 & 0.816574 & 52.40 \\
& $\sigma$ & 1 & 0 & 0.000017 & 0.000013 & 0.000053 & 0.000001 & 0.057681 & 1.73 \\
\hline SBPSO & $\bar{x}$ & 34 & 6 & 0.007670 & 0.000960 & 0.002334 & 0.000357 & 0.826267 & 26.87 \\
& $\sigma$ & 5 & 1 & 0.000319 & 0.000105 & 0.000527 & 0.000019 & 0.054478 & 1.63 \\
\hline
\end{tabular}

Table 4.5: DAX 100 rankings for each performance measure

\begin{tabular}{|c|c|c|c|c|c|c|c|c|c|c|}
\hline Algorithm & Result & $\mathbf{N}$ & $K$ & $R$ & $\bar{\sigma}$ & GD & IGD & HV & Time & Overall \\
\hline \multirow[t]{5}{*}{$\mathrm{ABC}$} & Wins & 4 & 0 & 4 & 0 & 3 & 4 & 0 & 3 & 18 \\
\hline & Losses & 0 & 4 & 0 & 4 & 0 & 0 & 4 & 1 & 13 \\
\hline & Draws & 0 & 0 & 0 & 0 & 1 & 0 & 0 & 0 & 1 \\
\hline & Difference & 4 & -4 & 4 & -4 & 3 & 4 & -4 & 2 & 5 \\
\hline & Rank & 1 & 5 & 1 & 5 & 1 & 1 & 5 & 2 & 2 \\
\hline \multirow[t]{5}{*}{ FA } & Wins & 0 & 1 & 0 & 4 & 0 & 0 & 2 & 0 & 7 \\
\hline & Losses & 3 & 3 & 4 & 0 & 4 & 4 & 1 & 4 & 23 \\
\hline & Draws & 1 & 0 & 0 & 0 & 0 & 0 & 1 & 0 & 2 \\
\hline & Difference & -3 & -2 & -4 & 4 & -4 & -4 & 1 & -4 & -16 \\
\hline & Rank & 4 & 4 & 5 & 1 & 4 & 5 & 2 & 5 & 4 \\
\hline \multirow[t]{5}{*}{ GA } & Wins & 2 & 2 & 2 & 2 & 2 & 2 & 4 & 2 & 18 \\
\hline & Losses & 2 & 2 & 2 & 1 & 2 & 2 & 0 & 2 & 13 \\
\hline & Draws & 0 & 0 & 0 & 1 & 0 & 0 & 0 & 0 & 1 \\
\hline & Difference & 0 & 0 & 0 & 1 & 0 & 0 & 4 & 0 & 5 \\
\hline & Rank & 3 & 3 & 3 & 2 & 2 & 3 & 1 & 3 & 2 \\
\hline \multirow[t]{5}{*}{ PSO } & Wins & 3 & 4 & 3 & 1 & 3 & 3 & 1 & 1 & 19 \\
\hline & Losses & 1 & 0 & 1 & 2 & 0 & 1 & 2 & 3 & 10 \\
\hline & Draws & 0 & 0 & 0 & 1 & 1 & 0 & 1 & 0 & 3 \\
\hline & Difference & 2 & 4 & 2 & -1 & 3 & 2 & -1 & -2 & 9 \\
\hline & Rank & 2 & 1 & 2 & 4 & 1 & 2 & 4 & 4 & 1 \\
\hline \multirow[t]{5}{*}{ SBPSO } & Wins & 0 & 3 & 1 & 1 & 1 & 1 & 1 & 4 & 12 \\
\hline & Losses & 3 & 1 & 3 & 1 & 3 & 3 & 1 & 0 & 15 \\
\hline & Draws & 1 & 0 & 0 & 2 & 0 & 0 & 2 & 0 & 5 \\
\hline & Difference & -3 & 2 & -2 & 0 & -2 & -2 & 0 & 4 & -3 \\
\hline & Rank & 4 & 2 & 4 & 3 & 3 & 4 & 3 & 1 & 3 \\
\hline
\end{tabular}

ABC found statistically significantly more unique portfolios than the other algorithms, with an average of 49 portfolios - seven more than the closest algo- 
rithm (i.e. PSO). Furthermore, the portfolios obtained by ABC contained statistically significantly more assets than the other algorithms, but by a smaller margin than for the Hang Seng benchmark problem. The GA and FA found portfolios with an average of 11 and 10 assets, respectively, which was roughly twice that of SBPSO and PSO. Although SBPSO was able to find small portfolios, it did not find as many optimal portfolios as ABC, PSO and the GA. Hence, SBPSO ranked last for the number of optimal portfolios obtained alongside the FA.

$\mathrm{ABC}$, PSO, the GA were able to approximate the true POF, as seen in Figure 4.2. The points on the POF obtained by PSO were more centered along the curve than the points obtained by ABC and the GA, which were more evenly distributed by comparison. Nonetheless, the PSO was as competitive as $\mathrm{ABC}$, and better than the GA, with regard to GD. Furthermore, the PSO ranked second in the IGD measure, outperforming the GA but losing to ABC. The FA failed to approximate the true POF as indicated by its scattered POF and low rankings for GD and IGD. The SBPSO was better than the FA at approximating the true POF, but was not as good as ABC, PSO and the GA. Furthermore, the average GD scores for FA and SBPSO were an order of magnitude worse than the other algorithms.

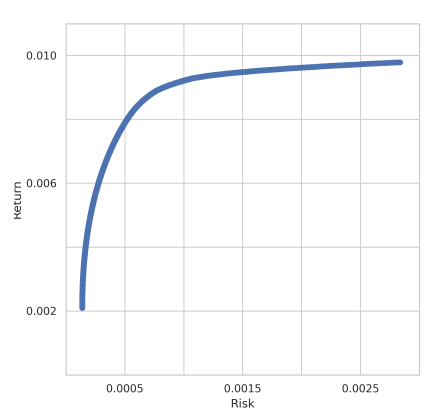

(a) True POF

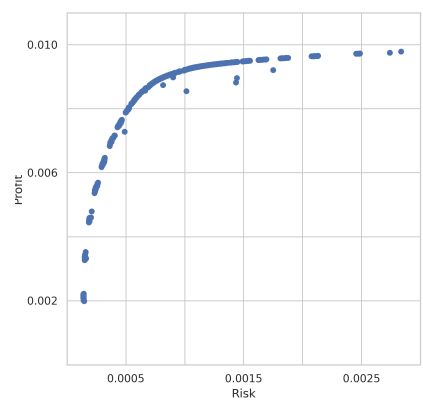

(d) GA

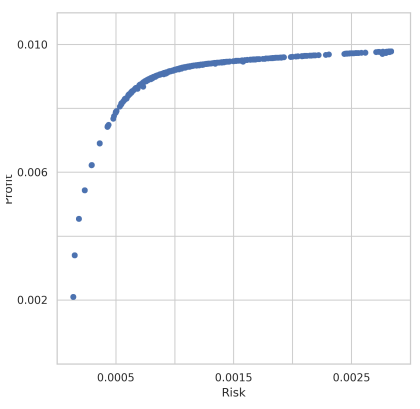

(b) $\mathrm{ABC}$

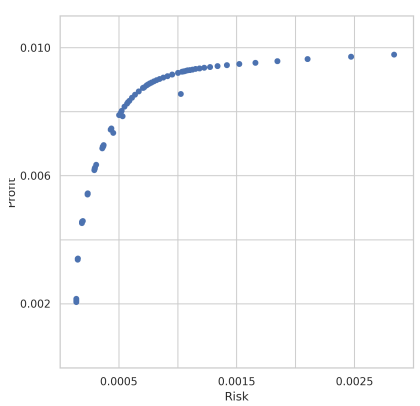

(e) PSO

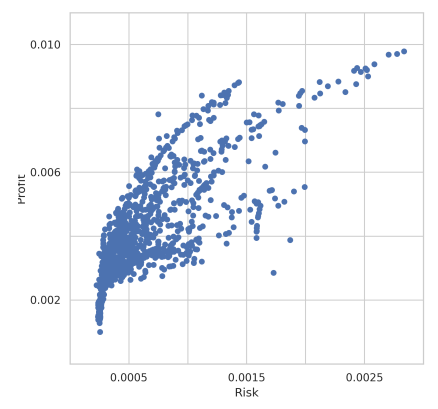

(c) FA

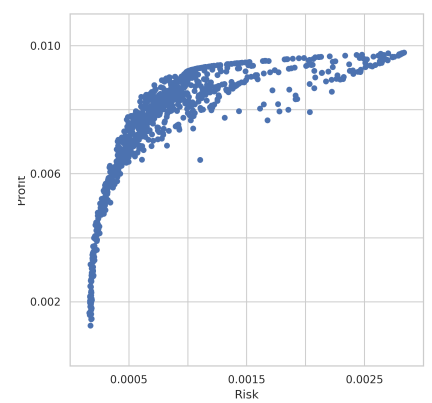

(f) SBPSO

Figure 4.2: Obtained Pareto-optimal fronts for DAX 100

SBPSO reached the stopping condition in 26.87 seconds, which was statis- 
tically significantly faster than the other algorithms. The ABC ranked second in time and reached the stopping condition in 43.68 seconds. The PSO and GA took around 50 seconds to reach the stopping condition. Lastly, the FA was the slowest algorithm with an average time of 166.26 seconds - at least three times slower than the other algorithms.

Overall, the PSO was the highest ranked algorithm for the DAX 100 benchmark problem. The ABC and GA tied for second place, while the SBPSO and FA ranked third and last, respectively.

\subsubsection{FTSE 100}

The performance of SBPSO, PSO, and the GA were very similar for portfoliorelated performance measures, as seen in Table 4.6. The return and risk results for the aforementioned algorithms were much the same. Furthermore, Table 4.7 shows that those algorithms ranked similarly for return and risk. ABC again ranked first for return and last for risk, and the FA ranked last for return and first for risk.

Table 4.6: FTSE 100 results for each performance measure

\begin{tabular}{lccccccccc}
\hline Algorithm & Result & $\mathbf{N}$ & $K$ & $R$ & $\bar{\sigma}$ & GD & IGD & HV & Time \\
\hline ABC & $\bar{x}$ & 49 & 16 & 0.007277 & 0.001073 & 0.000166 & 0.000232 & 0.679689 & 47.09 \\
& $\sigma$ & 1 & 1 & 0.000011 & 0.000005 & 0.000020 & 0.000000 & 0.020228 & 1.02 \\
\hline FA & $\bar{x}$ & 36 & 12 & 0.004745 & 0.000779 & 0.003347 & 0.000467 & 0.714994 & 186.78 \\
& $\sigma$ & 4 & 2 & 0.000445 & 0.000098 & 0.000356 & 0.000087 & 0.013245 & 25.15 \\
\hline GA & $\bar{x}$ & 29 & 12 & 0.006635 & 0.000778 & 0.000408 & 0.000238 & 0.766213 & 58.45 \\
& $\sigma$ & 1 & 1 & 0.000163 & 0.000029 & 0.000321 & 0.000024 & 0.035444 & 1.29 \\
\hline PSO & $\bar{x}$ & 29 & 6 & 0.006667 & 0.000782 & 0.000190 & 0.000232 & 0.704657 & 59.86 \\
& $\sigma$ & 0 & 0 & 0.000008 & 0.000001 & 0.000039 & 0.000001 & 0.049243 & 0.95 \\
\hline SBPSO & $\bar{x}$ & 33 & 6 & 0.006605 & 0.000822 & 0.001206 & 0.000264 & 0.725351 & 13.81 \\
& $\sigma$ & 6 & 2 & 0.000176 & 0.000061 & 0.000472 & 0.000017 & 0.037266 & 5.43 \\
\hline
\end{tabular}


CHAPTER 4. SET-BASED PARTICLE SWARM OPTIMIZATION FOR PORTFOLIO OPTIMIZATION

Table 4.7: FTSE 100 rankings for each performance measure

\begin{tabular}{|c|c|c|c|c|c|c|c|c|c|c|}
\hline Algorithm & Result & $\mathbf{N}$ & $K$ & $R$ & $\bar{\sigma}$ & GD & IGD & HV & Time & Overall \\
\hline \multirow[t]{5}{*}{$\mathrm{ABC}$} & Wins & 4 & 0 & 4 & 0 & 4 & 3 & 0 & 3 & 18 \\
\hline & Losses & 0 & 4 & 0 & 4 & 0 & 0 & 3 & 1 & 12 \\
\hline & Draws & 0 & 0 & 0 & 0 & 0 & 1 & 1 & 0 & 2 \\
\hline & Difference & 4 & -4 & 4 & -4 & 4 & 3 & -3 & 2 & 6 \\
\hline & Rank & 1 & 4 & 1 & 3 & 1 & 1 & 5 & 2 & 1 \\
\hline \multirow[t]{5}{*}{ FA } & Wins & 3 & 1 & 0 & 2 & 0 & 0 & 2 & 0 & 8 \\
\hline & Losses & 1 & 2 & 4 & 0 & 4 & 4 & 2 & 4 & 21 \\
\hline & Draws & 0 & 1 & 0 & 2 & 0 & 0 & 0 & 0 & 3 \\
\hline & Difference & 2 & -1 & -4 & 2 & -4 & -4 & 0 & -4 & -13 \\
\hline & Rank & 2 & 3 & 3 & 1 & 5 & 4 & 3 & 5 & 4 \\
\hline \multirow[t]{5}{*}{ GA } & Wins & 0 & 1 & 1 & 2 & 2 & 2 & 4 & 2 & 14 \\
\hline & Losses & 3 & 2 & 1 & 0 & 2 & 2 & 0 & 2 & 12 \\
\hline & Draws & 1 & 1 & 2 & 2 & 0 & 0 & 0 & 0 & 6 \\
\hline & Difference & -3 & -1 & 0 & 2 & 0 & 0 & 4 & 0 & 2 \\
\hline & Rank & 4 & 3 & 2 & 1 & 3 & 2 & 1 & 3 & 3 \\
\hline \multirow[t]{5}{*}{$\mathrm{PSO}$} & Wins & 0 & 3 & 1 & 2 & 3 & 3 & 0 & 1 & 13 \\
\hline & Losses & 3 & 1 & 1 & 0 & 1 & 0 & 2 & 3 & 11 \\
\hline & Draws & 1 & 0 & 2 & 2 & 0 & 1 & 2 & 0 & 8 \\
\hline & Difference & -3 & 2 & 0 & 2 & 2 & 3 & -2 & -2 & 2 \\
\hline & Rank & 4 & 2 & 2 & 1 & 2 & 1 & 4 & 4 & 3 \\
\hline \multirow[t]{5}{*}{ SBPSO } & Wins & 2 & 4 & 1 & 1 & 1 & 1 & 2 & 4 & 16 \\
\hline & Losses & 2 & 0 & 1 & 3 & 3 & 3 & 1 & 0 & 13 \\
\hline & Draws & 0 & 0 & 2 & 0 & 0 & 0 & 1 & 0 & 3 \\
\hline & Difference & 0 & 4 & 0 & -2 & -2 & -2 & 1 & 4 & 3 \\
\hline & Rank & 3 & 1 & 2 & 2 & 4 & 3 & 2 & 1 & 2 \\
\hline
\end{tabular}

As for the previous benchmarks, SBPSO and PSO obtained smaller portfolios than the other algorithms with fewer of the number of assets. The results show that $\mathrm{ABC}$ found 49 optimal portfolios on average, whereas the next best algorithm, the FA, found 13 fewer.

Figure 4.3 shows that $\mathrm{ABC}$ and PSO were able to approximate the true POF. The GA also performed well in approximating the true POF, but its obtained POF contains solutions that are dominated by the true POF. Further visual analysis shows that points of the POF obtained the FA were scattered and do not follow the trend of the true POF. The POF obtained by the SBPSO somewhat resembled the true POF, but it was not as close as ABC, PSO or the GA. The average GD and IGD scores for ABC were the best out of all algorithms, however, its average HV score was the worst. The poor HV score coincides with the concentration of points on the upper part of its obtained POF. 


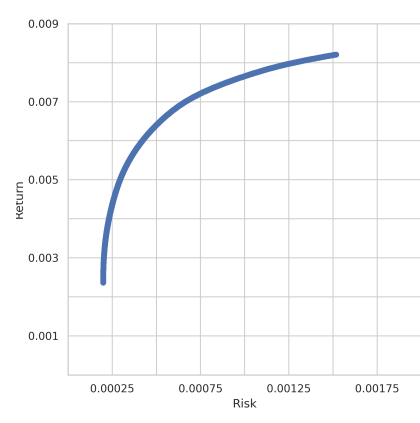

(a) True POF

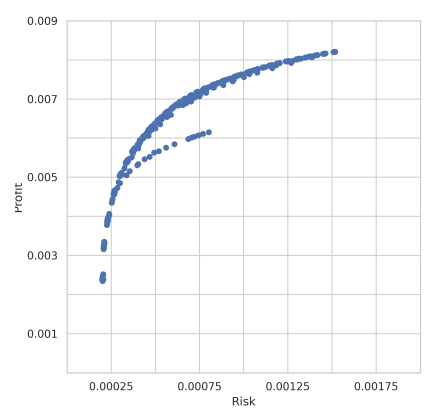

(d) GA

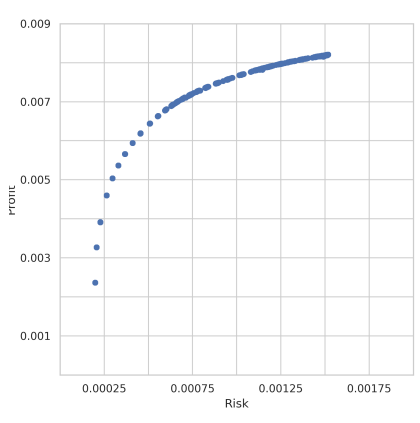

(b) $\mathrm{ABC}$

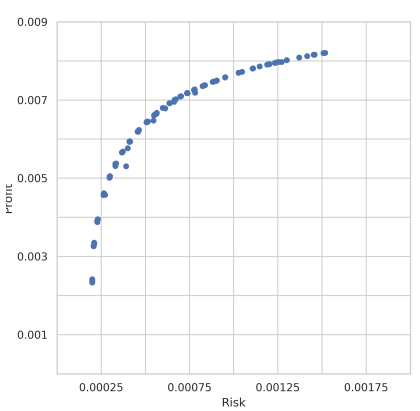

(e) PSO

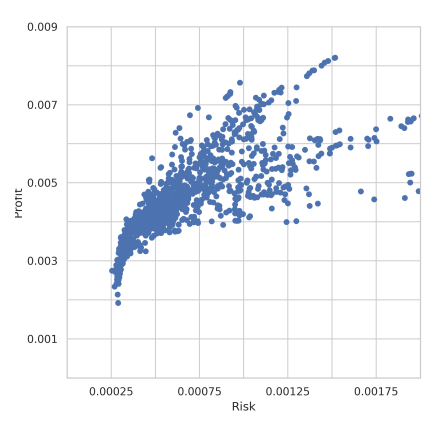

(c) FA

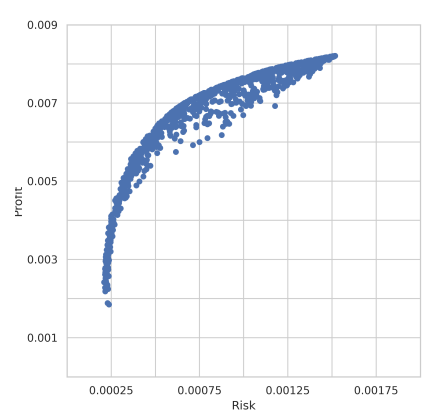

(f) SBPSO

Figure 4.3: Obtained Pareto-optimal fronts for FTSE 100

The fastest algorithm to reach the stopping condition was SBPSO, with an average time of 13.81 seconds. Furthermore, the search time of SBPSO decreased from the previous benchmark problem, despite the increase in problem size. Also, the SBPSO was approximately 13 times faster than the FA, the slowest algorithm. ABC was the second fastest algorithm with an average time to reach the stopping condition of 47.09 seconds, while PSO and the GA were approximately 10 seconds slower.

For the FTSE 100 benchmark problem, ABC was the best performing algorithm overall. The proposed SBPSO approach was the second best performing algorithm, while the GA and PSO tied for third place. The FA ranked last.

\subsubsection{S\&P 100}

Table 4.8 shows that $\mathrm{ABC}$ and PSO had the highest average return values for the S\&P benchmark problem, with ABC narrowly beating PSO. The rankings in Table 4.9 confirm this assessment. Furthermore, the average risk value of the portfolios found by PSO was lower than that of ABC. Thus, the PSO was more competitive for this benchmark problem than $\mathrm{ABC}$. The rankings and average values for return and risk show that SBPSO and the GA performed similarly. Both SBPSO and the GA were more profitable than the FA while being less risky than PSO and ABC. 
CHAPTER 4. SET-BASED PARTICLE SWARM OPTIMIZATION FOR PORTFOLIO OPTIMIZATION

Table 4.8: S\&P 100 results for each performance measure

\begin{tabular}{lccccccccc}
\hline Algorithm & Result & $\mathbf{N}$ & $K$ & $R$ & $\bar{\sigma}$ & GD & IGD & HV & Time \\
\hline ABC & $\bar{x}$ & 49 & 24 & 0.007843 & 0.001398 & 0.000236 & 0.000241 & 0.841527 & 56.54 \\
& $\sigma$ & 1 & 3 & 0.000031 & 0.000035 & 0.000031 & 0.000000 & 0.018891 & 1.21 \\
\hline FA & $\bar{x}$ & 38 & 18 & 0.005203 & 0.000891 & 0.003536 & 0.000535 & 0.825091 & 230.75 \\
& $\sigma$ & 5 & 2 & 0.000691 & 0.000246 & 0.000453 & 0.000093 & 0.016919 & 24.39 \\
\hline GA & $\bar{x}$ & 35 & 6 & 0.007430 & 0.001177 & 0.000810 & 0.000260 & 0.881914 & 54.52 \\
& $\sigma$ & 2 & 0 & 0.000088 & 0.000052 & 0.000265 & 0.000007 & 0.042699 & 1.31 \\
\hline PSO & $\bar{x}$ & 44 & 8 & 0.007705 & 0.001245 & 0.000269 & 0.000241 & 0.892730 & 64.55 \\
& $\sigma$ & 1 & 0 & 0.000010 & 0.000012 & 0.000096 & 0.000001 & 0.052169 & 1.26 \\
\hline SBPSO & $\bar{x}$ & 43 & 9 & 0.007269 & 0.001219 & 0.001775 & 0.000301 & 0.890120 & 35.69 \\
& $\sigma$ & 2 & 3 & 0.000428 & 0.000174 & 0.000620 & 0.000036 & 0.031804 & 5.56 \\
\hline
\end{tabular}

Table 4.9: S\&P 100 rankings for each performance measure

\begin{tabular}{|c|c|c|c|c|c|c|c|c|c|c|}
\hline Algorithm & Result & $\mathbf{N}$ & $K$ & $R$ & $\bar{\sigma}$ & GD & IGD & HV & Time & Overall \\
\hline \multirow[t]{5}{*}{$\mathrm{ABC}$} & Wins & 4 & 0 & 4 & 0 & 3 & 3 & 1 & 2 & 17 \\
\hline & Losses & 0 & 4 & 0 & 4 & 0 & 0 & 3 & 2 & 13 \\
\hline & Draws & 0 & 0 & 0 & 0 & 1 & 1 & 0 & 0 & 2 \\
\hline & Difference & 4 & -4 & 4 & -4 & 3 & 3 & -2 & 0 & 4 \\
\hline & Rank & 1 & 5 & 1 & 5 & 1 & 1 & 2 & 3 & 3 \\
\hline \multirow[t]{5}{*}{ FA } & Wins & 1 & 1 & 0 & 4 & 0 & 0 & 0 & 0 & 6 \\
\hline & Losses & 3 & 3 & 4 & 0 & 4 & 4 & 4 & 4 & 26 \\
\hline & Draws & 0 & 0 & 0 & 0 & 0 & 0 & 0 & 0 & 0 \\
\hline & Difference & -2 & -2 & -4 & 4 & -4 & -4 & -4 & -4 & -20 \\
\hline & Rank & 4 & 4 & 4 & 1 & 4 & 4 & 3 & 5 & 5 \\
\hline \multirow[t]{5}{*}{ GA } & Wins & 0 & 4 & 1 & 3 & 2 & 2 & 2 & 3 & 17 \\
\hline & Losses & 4 & 0 & 2 & 1 & 2 & 2 & 0 & 1 & 12 \\
\hline & Draws & 0 & 0 & 1 & 0 & 0 & 0 & 2 & 0 & 3 \\
\hline & Difference & -4 & 4 & -1 & 2 & 0 & 0 & 2 & 2 & 5 \\
\hline & Rank & 5 & 1 & 3 & 2 & 2 & 2 & 1 & 2 & 2 \\
\hline \multirow[t]{5}{*}{ PSO } & Wins & 3 & 3 & 3 & 1 & 3 & 3 & 2 & 1 & 19 \\
\hline & Losses & 1 & 1 & 1 & 3 & 0 & 0 & 0 & 3 & 9 \\
\hline & Draws & 0 & 0 & 0 & 0 & 1 & 1 & 2 & 0 & 4 \\
\hline & Difference & 2 & 2 & 2 & -2 & 3 & 3 & 2 & -2 & 10 \\
\hline & Rank & 2 & 2 & 2 & 4 & 1 & 1 & 1 & 4 & 1 \\
\hline \multirow[t]{5}{*}{ SBPSO } & Wins & 2 & 2 & 1 & 2 & 1 & 1 & 2 & 4 & 15 \\
\hline & Losses & 2 & 2 & 2 & 2 & 3 & 3 & 0 & 0 & 14 \\
\hline & Draws & 0 & 0 & 1 & 0 & 0 & 0 & 2 & 0 & 3 \\
\hline & Difference & 0 & 0 & -1 & 0 & -2 & -2 & 2 & 4 & 1 \\
\hline & Rank & 3 & 3 & 3 & 3 & 3 & 3 & 1 & 1 & 4 \\
\hline
\end{tabular}

The ABC and FA obtained much larger portfolios than the other algorithms with an average of 24 and 18 assets per portfolio, respectively. In comparison, the other algorithms had smaller portfolios with fewer than 10 assets. The portfolios obtained by the GA had an average of 6 assets, while the portfolios obtained by PSO and SBPSO had 8 and 9 assets, respectively. ABC found the highest number of optimal portfolios, i.e. 49, which was consistent with previous results. SBPSO and PSO found a similar number of optimal portfolios with averages of 44 and 43 , respectively. The GA ranked fourth for the number 
of optimal portfolios found with an average of 38. Lastly, the FA algorithm found 35 optimal portfolios, the least of all algorithms.

Both ABC and PSO ranked first in the GD and IGD performance measures. This tie for first place is consistent with the very close average GD scores between ABC and PSO, and their identical IGD scores. The PSO, however, ranked higher than $\mathrm{ABC}$, with regard to the $\mathrm{HV}$ measure. In fact, PSO, SBPSO and the GA all ranked first for the HV measure, while ABC ranked second and the FA ranked last. SBPSO failed to approximate the POF as well as the other algorithms, except the FA, as seen in Figure 4.4. Furthermore, the low GD and IGD rankings for SBPSO quantify its inability to approximate the true POF for the S\&P 100 benchmark problem. Yet again the FA produced a scattered POF that had little resemblance to the true POF.

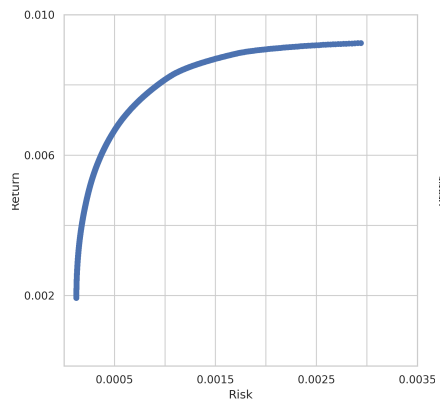

(a) True POF

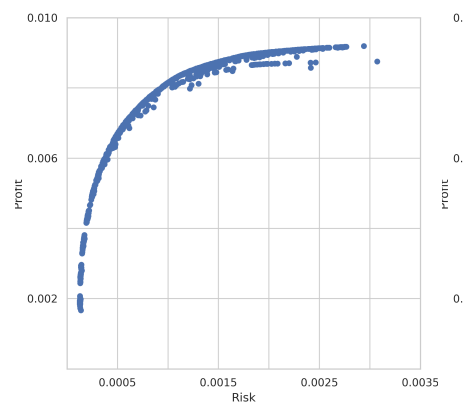

(d) GA

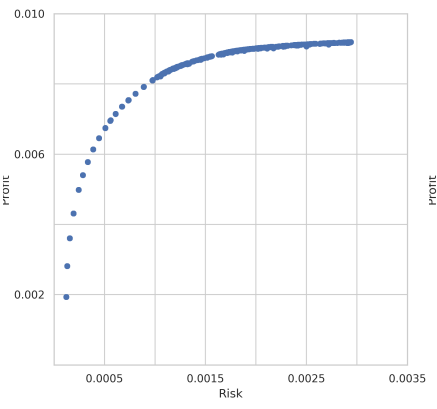

(b) $\mathrm{ABC}$

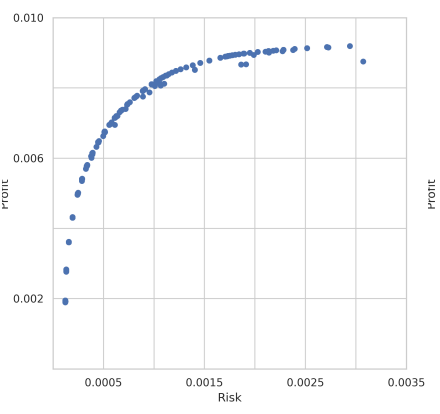

(e) PSO

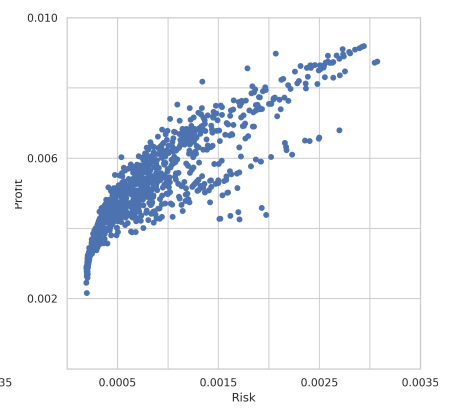

(c) FA

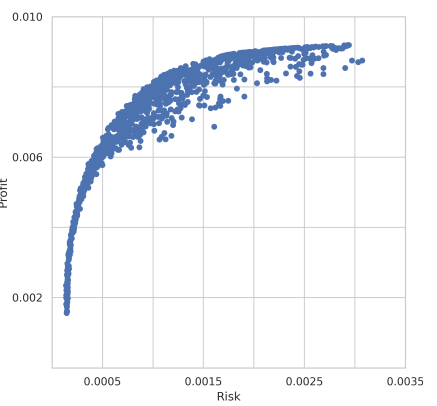

(f) SBPSO

Figure 4.4: Obtained Pareto-optimal fronts for the S\&P 100 benchmark

ABC, PSO and the GA took approximately a minute to reach the stopping condition, while the FA took almost four times that. SBPSO, the fastest algorithm thus far, reached the stopping condition in 35.69 seconds.

Overall, the PSO was the best performing algorithm for the S\&P benchmark problem. The second best performing algorithm was the GA, and the third was ABC. SBPSO ranked fourth overall and the FA ranked last. 


\subsubsection{Nikkei 225}

Table 4.11 shows that ABC ranked second for risk, while the FA ranked third the first time that ABC ranked higher for risk than the FA. Furthermore, ABC ranked first with regard to return, making it a very competitive algorithm for this benchmark problem. Another first was that the FA ranked last for risk, whereas the FA ranked first for risk on previous benchmark problems. Both the SBPSO and PSO also ranked first for risk. However, the PSO was statistically significantly more profitable than SBPSO. To elaborate, the portfolios obtained by $\mathrm{ABC}, \mathrm{PSO}$, and the GA were roughly 1.3 times more profitable than those obtained by SBPSO, while being relatively as risky, as shown in Table 4.10.

Table 4.10: Nikkei 225 results for each performance measure

\begin{tabular}{lccccccccc}
\hline Algorithm & Result & $\mathbf{N}$ & $K$ & $R$ & $\bar{\sigma}$ & GD & IGD & HV & Time \\
\hline ABC & $\bar{x}$ & 49 & 24 & 0.003348 & 0.000858 & 0.000470 & 0.000210 & 0.924789 & 254.99 \\
& $\sigma$ & 0 & 6 & 0.000022 & 0.000015 & 0.000221 & 0.000001 & 0.046806 & 2.91 \\
\hline FA & $\bar{x}$ & 28 & 12 & 0.001086 & 0.000962 & 0.004191 & 0.000490 & 0.734680 & 1087.28 \\
& $\sigma$ & 6 & 7 & 0.000484 & 0.000114 & 0.000561 & 0.000056 & 0.064953 & 117.14 \\
\hline GA & $\bar{x}$ & 46 & 22 & 0.003185 & 0.000858 & 0.001702 & 0.000276 & 0.906150 & 248.82 \\
& $\sigma$ & 4 & 3 & 0.000105 & 0.000068 & 0.000344 & 0.000019 & 0.043527 & 2.82 \\
\hline PSO & $\bar{x}$ & 44 & 7 & 0.003264 & 0.000782 & 0.000703 & 0.000218 & 0.957354 & 266.89 \\
& $\sigma$ & 2 & 0 & 0.000023 & 0.000022 & 0.000126 & 0.000008 & 0.037560 & 4.40 \\
\hline SBPSO & $\bar{x}$ & 42 & 10 & 0.002454 & 0.000800 & 0.002396 & 0.000353 & 0.826649 & 29.22 \\
& $\sigma$ & 4 & 5 & 0.000592 & 0.000065 & 0.000535 & 0.000065 & 0.067572 & 4.19 \\
\hline
\end{tabular}

All algorithms found a high number of optimal portfolios in comparison with previous benchmark problems, except for the FA. The portfolios obtained by the ABC and FA contained statistically significantly more assets than the GA, PSO and SBPSO - with portfolios which contained fewer than 10 assets.

Figure 4.5 shows that all algorithms struggled to approximate the true POF, although PSO and ABC were the closest. Rankings for the Pareto optimality measures support this statement, with ABC ranking first for GD and IGD, and second for HV. The PSO ranked second for GD and IGD, and first for HV. The GA performed relatively well, ranking third for all the Pareto optimality measures. SBPSO, however, ranked fourth for all Pareto optimality measures and was only statistically significantly better than the FA. 
CHAPTER 4. SET-BASED PARTICLE SWARM OPTIMIZATION FOR PORTFOLIO OPTIMIZATION

Table 4.11: Nikkei 225 rankings for each performance measure

\begin{tabular}{|c|c|c|c|c|c|c|c|c|c|c|}
\hline Algorithm & Result & $\mathbf{N}$ & $K$ & $R$ & $\bar{\sigma}$ & GD & IGD & HV & Time & Overall \\
\hline \multirow[t]{5}{*}{$\mathrm{ABC}$} & Wins & 4 & 0 & 4 & 1 & 4 & 4 & 2 & 2 & 21 \\
\hline & Losses & 0 & 4 & 0 & 2 & 0 & 0 & 0 & 2 & 8 \\
\hline & Draws & 0 & 0 & 0 & 1 & 0 & 0 & 2 & 0 & 3 \\
\hline & Difference & 4 & -4 & 4 & -1 & 4 & 4 & 2 & 0 & 13 \\
\hline & Rank & 1 & 4 & 1 & 2 & 1 & 1 & 2 & 3 & 1 \\
\hline \multirow[t]{5}{*}{ FA } & Wins & 0 & 2 & 0 & 0 & 0 & 0 & 0 & 0 & 2 \\
\hline & Losses & 4 & 1 & 4 & 4 & 4 & 4 & 4 & 4 & 29 \\
\hline & Draws & 0 & 1 & 0 & 0 & 0 & 0 & 0 & 0 & 1 \\
\hline & Difference & -4 & 1 & -4 & -4 & -4 & -4 & -4 & -4 & -27 \\
\hline & Rank & 4 & 2 & 5 & 3 & 5 & 5 & 5 & 5 & 4 \\
\hline \multirow[t]{5}{*}{ GA } & Wins & 3 & 1 & 2 & 1 & 2 & 2 & 2 & 3 & 16 \\
\hline & Losses & 1 & 3 & 2 & 2 & 2 & 2 & 1 & 1 & 14 \\
\hline & Draws & 0 & 0 & 0 & 1 & 0 & 0 & 1 & 0 & 2 \\
\hline & Difference & 2 & -2 & 0 & -1 & 0 & 0 & 1 & 2 & 2 \\
\hline & Rank & 2 & 3 & 3 & 2 & 3 & 3 & 3 & 2 & 2 \\
\hline \multirow[t]{5}{*}{$\mathrm{PSO}$} & Wins & 1 & 4 & 3 & 3 & 3 & 3 & 3 & 1 & 21 \\
\hline & Losses & 2 & 0 & 1 & 0 & 1 & 1 & 0 & 3 & 8 \\
\hline & Draws & 1 & 0 & 0 & 1 & 0 & 0 & 1 & 0 & 3 \\
\hline & Difference & -1 & 4 & 2 & 3 & 2 & 2 & 3 & -2 & 13 \\
\hline & Rank & 3 & 1 & 2 & 1 & 2 & 2 & 1 & 4 & 1 \\
\hline \multirow[t]{5}{*}{ SBPSO } & Wins & 1 & 2 & 1 & 3 & 1 & 1 & 1 & 4 & 14 \\
\hline & Losses & 2 & 1 & 3 & 0 & 3 & 3 & 3 & 0 & 15 \\
\hline & Draws & 1 & 1 & 0 & 1 & 0 & 0 & 0 & 0 & 3 \\
\hline & Difference & -1 & 1 & -2 & 3 & -2 & -2 & -2 & 4 & -1 \\
\hline & Rank & 3 & 2 & 4 & 1 & 4 & 4 & 4 & 1 & 3 \\
\hline
\end{tabular}

For Nikkei 225, the largest benchmark problem, the FA took approximately 1000 seconds to reach the stopping condition - an order of magnitude worse than ABC, PSO and the GA, and two orders of magnitude worse than SBPSO. In contrast, $\mathrm{ABC}, \mathrm{PSO}$ and the GA took around 250 seconds to reach the stopping condition.

PSO was the highest ranked algorithm overall for the Nikkei 225 benchmark problem. The GA ranked second overall and the $\mathrm{ABC}$ third. However, the difference in performance by the $\mathrm{GA}$ and $\mathrm{ABC}$ is small as the $\mathrm{ABC}$ incurred only one more loss than the GA. The SBPSO ranked fourth overall, and the FA ranked last. 


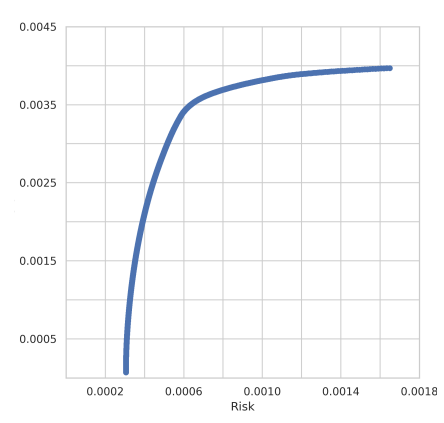

(a) True POF

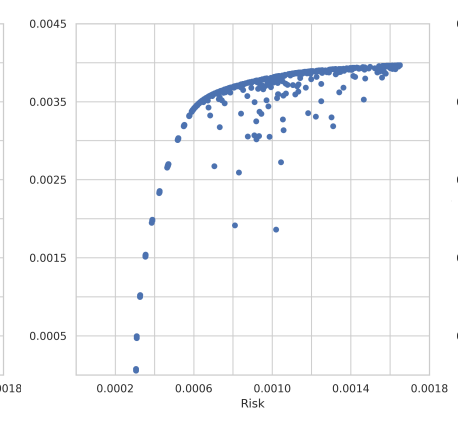

(b) $\mathrm{ABC}$

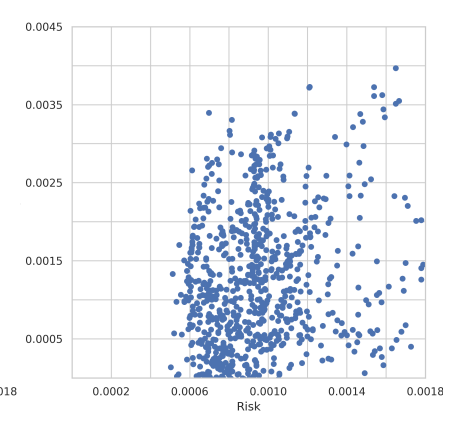

(c) FA

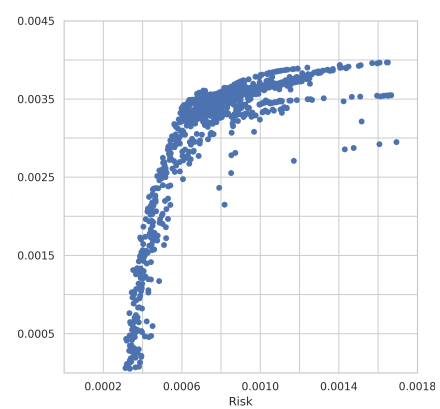

(d) GA

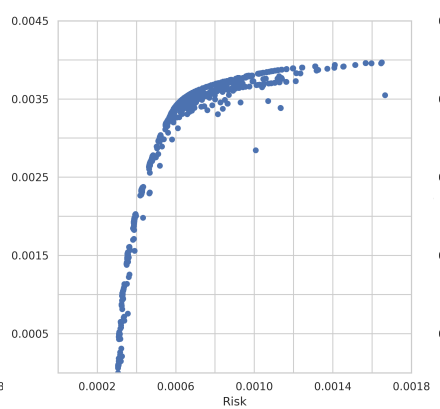

(e) PSO

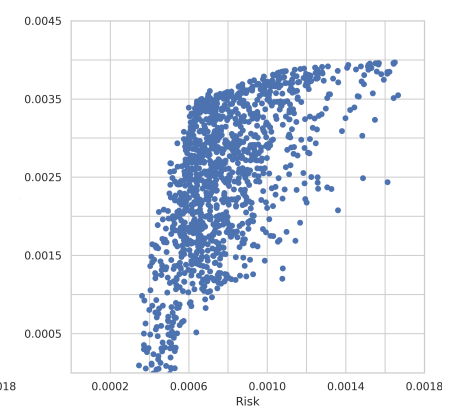

(f) SBPSO

Figure 4.5: Obtained Pareto-optimal fronts for the Nikkei 225 benchmark

\subsection{Summary}

This chapter proposed a set-based particle swarm optimization (SBPSO) for the mean-variance portfolio optimization problem. The SBPSO is used for asset selection while a particle swarm optimization (PSO) algorithm is used for asset weight determination. A thorough investigation into the performance of the SBPSO performance was undertaken. This included comparing the SBPSO with four known algorithms for portfolio optimization, namely artificial bee colony $(\mathrm{ABC})$, firefly algorithm (FA), PSO and a genetic algorithm (GA), on five benchmark problems. ABC was the best performing algorithm overall. Furthermore, it was fast, had higher return values and obtained statistically significantly more optimal portfolios than the other algorithms. The performance of the PSO was close to that of ABC and the PSO found smaller sized portfolios than the other algorithms. The GA was the third best performing algorithm overall. The performance of the proposed SBPSO was better than the FA and close to the GA. Furthermore, the ability of SBPSO to approximate the true POF deteriorated as the dimensionality of the portfolio problems increased. However, the SBPSO was statistically significantly faster than the other algorithms, and was consistent in its time to reach the stopping condition, despite the dimensionality of the problems increasing. The FA was 
CHAPTER 4. SET-BASED PARTICLE SWARM OPTIMIZATION FOR PORTFOLIO OPTIMIZATION

the worst performing algorithm, and failed to approximate the true POF for all portfolio problems.

The SBPSO results show that is possible to obtain good quality portfolios quickly by treating portfolio optimization as a set-based problem. However, SBPSO did not perform as well as PSO and ABC. It is hypothesized that this gap in performance is due to the inadequate time SBPSO allocates to optimize the weights for a candidate solution. Furthermore, the performance of SBPSO can be better understood by examining its exploration and exploitation behaviour. Chapter 6 further investigates the performance of SBPSO for portfolio optimization and suggests improvements. 


\section{Chapter 5}

\section{Set-based Diversity Measures}

Chapter 4 showed that SBPSO obtains good-quality solutions while being relatively fast. However, SBPSO performed worse than the ABC, PSO and GA in its ability to approximate the POF. Insight into the exploration and exploitation behaviour of SBPSO will help us understand its performance for portfolio optimization. This insight can be gained by measuring the diversity of the particles moving through a search space. Much of the work done on quantifying diversity has focused on heuristics for continuous-valued search spaces [134]. Due to the fact that sets are not of the same size, and since all existing diversity measures assume same size candidate solutions, these measures are not applicable to set-based algorithms. Furthermore, sets lack spatial structure in that distance, in the traditional sense, cannot be measured between two sets. Therefore, there is a need for set-based diversity measures that give researchers the same insights for set-based heuristics as the diversity measures for heuristics that require candidate solutions to be of the same size. The objective of this chapter 1 is to propose diversity measures for set-based optimization algorithms. To do this, a thorough investigation into the use of the Jaccard distance and the Hamming distance as methods for calculating the diversity of set-based populations is conducted.

The rest of this chapter is organized as follows: Section 5.1 introduces the reader to diversity concepts and existing diversity measures. Section 5.2 formally defines the Jaccard distance and the Hamming distance. Numerical examples demonstrating these distance measures as set-based diversity measures are given in Section 5.3. Section 5.4 describes the empirical process used to validate the two set-based diversity measures. Section 5.5 presents and discusses the results. Section 5.6 summarises the findings.

\footnotetext{
${ }^{1}$ This chapter was published in the 7 th International Conference on Soft Computing Machine Intelligence under the title "Diversity measures for set-based meta-heuristics" [53].
} 


\section{$5.1 \quad$ Introduction}

The diversity of a population of agents over time allows a researcher to assess the ability of the heuristic to sufficiently explore the search space (initially), later to refine (exploit) potentially good solutions. Low diversification indicates that the agents are refining a potentially good solution, while high diversification shows that the agents are exploring the search space. If diversity is low throughout the search, modifications can then be made to the heuristic to influence the agents to spend more time exploring other areas of the search space. Likewise, if the diversity is constantly high, changes can then be made to promote exploitation of fruitful areas. If the distance measures are normalised by search space bounds, then a diversity value of zero indicates that all agents in the population have converged to the same point in the search space. Conversely, a diversity value of one means that the agents are totally different from one other. If the distance measures are not normalised by search space bounds, then the maximum diversity is a function of the largest distance between extreme points in the search space. A researcher expects a that the value of a diversity measure to be at a maximum when the agents differ completely and that the value decreases as the agents become more similar. Furthermore, a researcher expects that when the agents are identical the value of a diversity measure is zero.

The diversity of population-based algorithms, like particle swarm optimization (PSO) [49], [52] and genetic algorithms (GAs) [52], [85], can be quantified using various methods typically associated with the Euclidean distance [134. For example, the average Euclidean distance between each individual or the average distance from each individual to the epicenter of the population can be considered the diversity of the population 134. However, no work has been done on measuring the diversity of set-based algorithms.

Existing diversity measures are not suitable for set-based populations, because sets can differ in size and there is no concept of distance between elements in the sets. This chapter proposes two set-based diversity measures; one based on the Jaccard distance 83 and the other based on the Hamming distance 73 .

\subsection{Set-based Distance Measures}

This section defines the Jaccard distance and Hamming distance, both of which are well-established non-Euclidean distance measures for discrete-valued representations. Section 5.2.1 defines the Jaccard distance and Section 5.2 .2 defines the Hamming distance. 


\subsubsection{Jaccard Distance}

The Jaccard index measures the similarity between two sets as the cardinality of their intersection divided by the cardinality of their union [83. Formally, the Jaccard index, also known as the Jaccard similarity coefficient, is

$$
J(A, B)=\frac{A \cap B}{A \cup B}
$$

where $A$ and $B$ are two sets.

The Jaccard distance is simply the complement of the Jaccard index and is calculated as

$$
d_{J}(A, B)=1-\frac{A \cap B}{A \cup B}
$$

The Jaccard distance is equal to 1.0 when $A$ and $B$ are disjoint. Moreover, when $A$ and $B$ are identical, $d_{J}$ is equal to 0.0 . The Jaccard distance is suited for measuring diversity, since sets in a given collection that are disjoint will have an average $d_{j}$ of 1.0. As these sets become more similar to one another, the average $d_{J}$ value will approach 0.0 .

\subsubsection{Hamming Distance}

The Hamming distance is an indication of the minimum number of substitutions required to change one string into another string of equal length, by counting the number of positions where corresponding symbols differ $73 \mid$. This can generally be applied to vectors of equal length, so long as the vectors are bit vectors. The Hamming distance between vectors $\boldsymbol{u}$ and $\boldsymbol{v}$, each of length $n$, is calculated as

$$
d_{H}(\boldsymbol{u}, \boldsymbol{v})=\frac{d_{H}^{\prime}(\boldsymbol{u}, \boldsymbol{v})}{n_{x}}
$$

where

$$
d_{H}^{\prime}(\boldsymbol{u}, \boldsymbol{v})=\sum_{j=1}^{n_{x}} \iota\left(u_{j}, v_{j}\right)
$$

and

$$
\iota(u, v)=\left\{\begin{array}{l}
1 \text { if } u \neq v \\
0 \text { if } u=v
\end{array}\right.
$$

The Hamming distance produces a maximum value of 1.0 when all components between $\boldsymbol{u}$ and $\boldsymbol{v}$ are in disagreement. The Hamming distance is 0.0 when all the components are in agreement.

\subsection{Diversity Measures for Sets}

This section proposes the Jaccard and Hamming diversity measures for setbased heuristics, and showcases the difference between the two. Jaccard diversity is defined in Section 5.3.1 and Hamming diversity is defined in Section 
5.3 .2 . Section 5.3 .3 gives a series of examples of calculating the Jaccard and Hamming diversity measures.

\subsubsection{Jaccard Diversity}

Jaccard diversity is the average Jaccard distance between all set pairs in a given population, $S$. Formally, Jaccard diversity is calculated as

$$
\bar{d}_{J}=\frac{\sum_{i}^{n-1} \sum_{j=i+1}^{n} d_{J}\left(S_{i}, S_{j}\right)}{\sum_{i}^{n-1} \sum_{j=i+1}^{n} 1}
$$

where $n$ is the cardinality of $S$, and $S_{i}$ and $S_{j}$ are the sets corresponding to indices $i$ and $j$, respectively.

\subsubsection{Hamming Diversity}

Hamming diversity is the average Hamming distance between all set pairs in $S$. Note that the Hamming distance is defined for vectors of the same length of discrete valued elements. In order to apply the Hamming distance to sets, set-based representations have to converted to vector-based representations. This is done by applying a mapping function, $\omega$, to the set.

$$
\omega: S \subseteq U \rightarrow B^{n}
$$

where $B=\{0,1\}$.

Given $S=\left\{u_{1}, u_{2}, \cdots, u_{n}\right\}$, the corresponding bit vector is

$$
\omega(S)=\left(V_{1}, V_{2}, \cdots, V_{n}\right)
$$

where

$$
V_{j}=\left\{\begin{array}{l}
1 \text { if } U_{j} \in S \\
0 \text { otherwise }
\end{array}\right.
$$

The following illustrates the use of $\omega$ to convert the set $\{1,2,5\}$ in the universe of the first five natural numbers to a bit vector. The length of the bit vector is the size of the universe. Each index in the bit vector corresponds to an element in the universe. For each element in the set $\{1,2,5\}$, the corresponding bit vector values are set to 1 and all other values are set to 0 , since they are not present in the set. Therefore, the bit vector repesentation of set $\{1,2,5\}$ is

$$
\omega(\{1,2,5\})=(1,1,0,0,1)
$$

Diversity using Hamming distance, or simply, Hamming diversity is

$$
\bar{d}_{H}=\frac{\sum_{i}^{n-1} \sum_{j=i+1}^{n} d_{H}\left(\omega\left(S_{i}\right), \omega\left(S_{j}\right)\right)}{\sum_{i}^{n-1} \sum_{j=i+1}^{n} 1}
$$


One immediate downside to the Hamming diversity is that each set has to be converted to a larger $|U|$-dimensional vector where $U$ is the set universe.

\subsubsection{Examples}

The purpose of this section is to illustrate the application of the diversity measures on special cases. The first two examples demonstrate how to calculate the Jaccard and Hamming diversity of sets. The remaining examples show sets that start at maximum diversity progressively moving to a minimum diversity. Furthermore, the results of the examples are discussed.

\subsubsection{Example 1 - Jaccard Diversity}

Consider the collection of sets, $S=(\{1\},\{3\},\{1,2\})$. The Jaccard diversity, $\bar{d}_{J}$, of $S$ is calculated as follows:

$$
\begin{gathered}
d_{J}(\{1,2\},\{1\})=1-\frac{|\{1,2\} \cap\{1\}|}{|\{1,2\} \cup\{1\}|}=0.5 \\
d_{J}(\{1,2\},\{3\})=1-\frac{|\{1,2\} \cap\{3\}|}{|\{1,2\} \cup\{3\}|}=1.0 \\
d_{J}(\{1\},\{3\})=1-\frac{|\{1\} \cap\{3\}|}{|\{1\} \cup\{3\}|}=1.0 \\
\therefore \bar{d}_{J}=\frac{0.5+1.0+1.0}{3}=0.8334
\end{gathered}
$$

This can be interpreted as the sets within $S$ are $83.34 \%$ dissimilar, or, simply, $16.67 \%$ similar. This outcome makes sense as there is ony one element that is shared and it is only shared between two of the three sets.

\subsubsection{Example 2 - Hamming Diversity}

The Hamming diversity, $\bar{d}_{H}$, of $S$, where $S$ contains $(\{1\},\{3\},\{1,2\})$, is calculated as

$$
\begin{gathered}
U=\{1,2,3\} \\
\omega(\{1\})=(1,0,0) \\
\omega(\{3\})=(0,0,1) \\
\omega(\{1,2\})=(1,2,0) \\
d_{H}((1,0,0),(1,1,0))=0 . \dot{3} \\
d_{H}((1,1,0),(0,0,1))=1.0 \\
d_{H}((1,0,0),(0,0,1))=0.6 \dot{6} \\
\therefore \bar{d}_{H}=\frac{0.3 \dot{3}+1.0+0.6 \dot{6}}{3}=0.6667
\end{gathered}
$$


Here the sets are $66.67 \%$ dissimilar, or $33.33 \%$ similar. This is an over representation of how similar the sets are, since there is no common element shared by all three sets.

\subsubsection{Example 3 - Sets at Maximum Diversity}

The rest of the examples in this section are all based on the scenario in which three set-based agents attempt to converge to a known optimum of $\{1,2\}$ in a set universe that contains the first 10 natural numbers, i.e. $U=\{1,2, \cdots, 10\}$. The search space consists of all permutations of set elements of all possible cardinalities. Each agent is initialized such that the diversity of the population $S$ is at a maximum by ensuring the elements of each agent is unique. The agents then move progressively closer towards the optimal set by changing one element at a time to better resemble the optimal set. The purpose of these examples is to mimic a scenario wherein diverse agents move over a number of iterations to collapse on a target set, i.e. $\{1,2\}$. Furthermore, these examples serve to establish a sense of the proposed measures.

The first example in this scenario uses

$$
S_{0}=(\{1,3,10\},\{4,6,8\},\{2,5,7,9\})
$$

where $S_{t}$ is the collection of sets at time step $t$. Each set in $S_{t}$ corresponds to an agent. Through inspection, the reader can deduce that the contents of each set differs from one another. Furthermore, it is expected that proposed setbased diversity measures will yield a value of 1.0, since the sets are completely different. The diversity measures are as follows

$$
\begin{gathered}
\bar{d}_{J}\left(S_{0}\right)=1.0 \\
\bar{d}_{H}\left(S_{0}\right)=0.6667
\end{gathered}
$$

This suggests that the Hamming diversity underrepresents diversity, because a value of 1.0 was expected.

\subsubsection{Example 4 - Sets That Are Slightly Similar}

This example shows the set-based agents from the previous example beginning to converge to the optimal set. Here, $S_{1}$ is $(\{1,2,10\},\{1,6\},\{2,5,7\})$. The diversity measures are

$$
\begin{gathered}
\bar{d}_{J}\left(S_{1}\right)=0.85 \\
\bar{d}_{H}\left(S_{1}\right)=0.4
\end{gathered}
$$

Both the Jaccard diversity and Hamming diversity values decreased from the previous time step, as expected, because it is assumed that the diversity values will decrease as the sets become more similar. 


\subsubsection{Example 5 - Sets That Are Extremely Similar}

Two of the set-based agents are shown to have converged on the optimal set, with $S_{2}=(\{1,2\},\{1,2\},\{1,2,7\})$. The values for the diversity measures are

$$
\begin{aligned}
& \bar{d}_{J}\left(S_{2}\right)=0.2221 \\
& \bar{d}_{H}\left(S_{2}\right)=0.0667
\end{aligned}
$$

Alternatively, this can be read as the sets within $S_{2}$ are $22.24 \%$ dissimilar according to $\bar{d}_{J}$ or $6.67 \%$ according to $\bar{d}_{H}$. The decrease in diversity values is expected since the sets have become more similar.

\subsubsection{Example 6 - Sets at Minimum Diversity}

The last example shows that all the set-based agents have converged on the optimal set. Here, $S_{3}$ contains $(\{1,2\},\{1,2\},\{1,2\})$. The Jaccard diversity and the Hamming diversity values are

$$
\begin{aligned}
& \bar{d}_{J}\left(S_{3}\right)=0.0 \\
& \bar{d}_{H}\left(S_{3}\right)=0.0
\end{aligned}
$$

Both values are in agreement that $S_{3}$ has converged, as expected.

\subsection{Empirical Process}

An empirical process that mimics set-based agents moving through a search is used to compare the proposed diversity measures. The empirical process involves two experiments, both of which move sets closer to a randomly generated optimal set. Furthermore, the first experiment focuses on investigating the effect of agent size with respect to diversity. The second experiment focuses on the size of the search space. The diversity values for each time step are recorded over 30 independent runs and are graphed.

\subsubsection{Experiment 1 - Agent Size}

In this experiment, set-based agents are randomly initialized such that each element only appears once in any set, so that the intersection between all sets is empty. This maximises the diversity of the population. An additional set, referred to as the optimal set, is also randomly initialized. Agents are updated at each time step by randomly selecting one of two options: The first is to randomly remove an element not within the optimal set. The second option is to add a randomly chosen element from the optimal set to the agent if the element is not already present. If one of these options is not applicable, then the other is used. If neither of the options are applicable, then the agent is equal 
to the optimal set. Thus, agents move closer, or in other words, become more similar, to the optimal set after each time step. This process is repeated until all sets are the same, giving a minimum diversity. It is therefore expected that the diversity decreases from the maximum diversity to the minimum diversity.

Three different agent sizes were used, i.e. small, large, and random (from a uniform distribution in [1,10]). For small, a size of 3 was used and for large, 10. Furthermore, each of the agent sizes, referred to as $n_{A}$ where $A$ is the agent, is paired with three different optimal set sizes. The sizes for the optimal set, $Y^{*}$, were 1,5 and 10 . The optimal set size is denoted as $n_{Y^{*}}$. Thus, a total of nine artificial scenarios were tested. For each scenario, a population of 10 was used, and a universe of 100 elements, i.e. $U=\{x \in \mathbb{R} \mid 1 \leq x \leq 100\}$.

\subsubsection{Experiment 2 - Universe Size}

Experiment 2 follows the same procedure as experiment 1. However, the effect of universe size is investigated instead of agent size. To accommodate this, elements can appear more than once during the initialisation process and each set is randomly sized. Thus, the initial agents can, to some degree, be similar. Three different universe sizes, $n_{U}$, are paired with the same $n_{Y^{*}}$ configurations from the previous experiment. The universe sizes considered were 10, 50, and 100 .

\subsection{Results}

The diversity profiles for all experiment 1 scenarios in Figure 5.1 show that Hamming diversity under-represents diversity, despite the contents of the sets on initialization being entirely different. The diversity, as a researcher would expect, should be at a maximum at the beginning of the search. The underrepresentation was caused by taking the size of the set universe into account when calculating diversity. For example, sets $\{1\}$ and $\{2\}$ in the universe of the first 10 natural numbers, i.e. $\{1,2, \cdots, 10\}$, were different in the sense that the contents were different. However, they were both similar in the sense that each excludes nine other elements. On the other hand, the Jaccard diversity was 1.0 for all initial sets in Figure 5.1. Each figure shows a smooth curve for Jaccard diversity, except for Figures 5.1(a) and 5.1(d) which show sharp drops in diversity as the population collapses to the single element optimal set. The sharp drops show that changes in populations with relatively small sized agents can drastically affect Jaccard diversity values. In comparison, the trends for the Jaccard diversity values of larger sets were much smoother. Hamming diversity shows small decreases in diversity at each time step, but maintained a comparatively low diversity throughout the search. The diversity trends for the Jaccard diversity were therefore as expected, while the Hamming diversity underestimated the diversity of the population. 
The second experiment shows similar results, as seen in Figure 5.2. The diversity values based on the Hamming distance were larger than for experiment 1, yet still smaller than the values based on the Jaccard distance. Furthermore, the trends for Hamming diversity were very similar across all figures. Jaccard diversity generally produced smooth trends with varying maximum diversity values. Figure 5.2(b) shows somewhat larger jumps in diversity than the other graphs for this experiment. However, the jumps were not as drastic as the worst cases of experiment 1. There is an initial uptick in diversity in the second experiment, unlike in the second experiment where diversity started at a maximum and then decreased. This uptick is due to the fact that the agents in the second experiment share similarity on initialization and then briefly become dissimilar as elements not within the optimal set are removed. Overall, the trends for each diversity measure capture the changes in diversity as the agents collapse on the optimal set, regardless of the size of the search space.

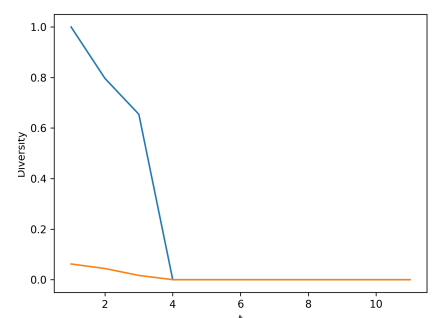

(a) $n_{A}=3, n_{Y^{*}}=1$

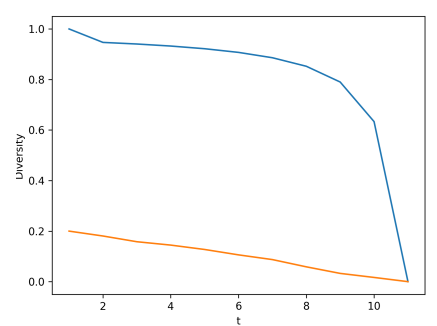

(d) $n_{A}=10, n_{Y^{*}}=1$

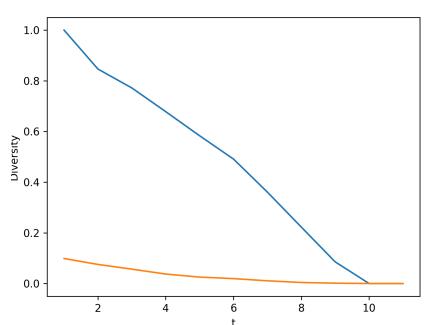

(g) $n_{A}=r, n_{Y^{*}}=1$

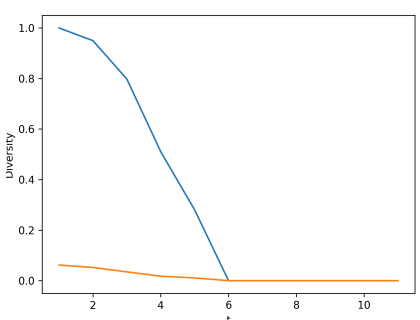

(b) $n_{A}=3, n_{Y^{*}}=5$

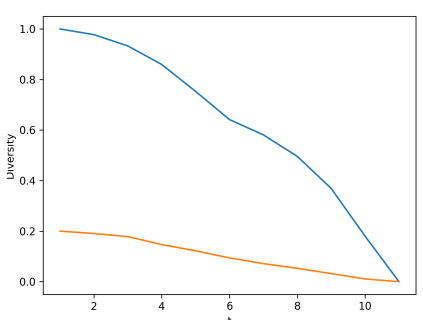

(e) $n_{A}=10, n_{Y^{*}}=5$

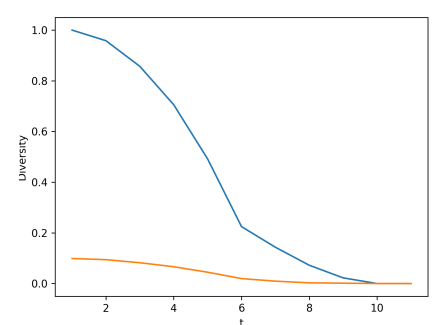

(h) $n_{A}=r, n_{Y^{*}}=5$

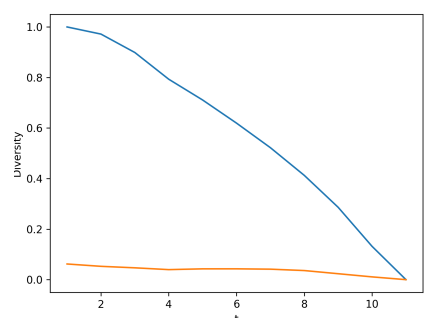

(c) $n_{A}=3, n_{Y^{*}}=10$

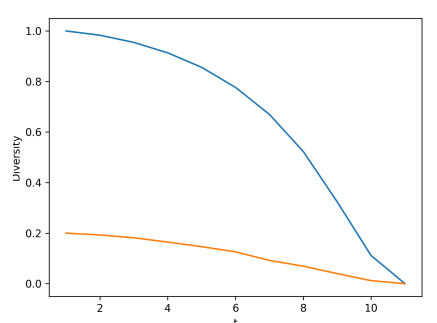

(f) $n_{A}=10, n_{Y^{*}}=10$

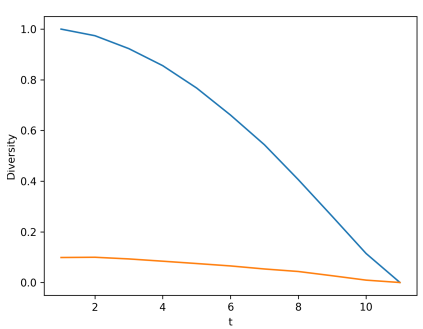

(i) $n_{A}=r, n_{Y^{*}}=10$

Figure 5.1: Jaccard Diversity (blue) and Hamming Diversity (orange) for Experiment 1 


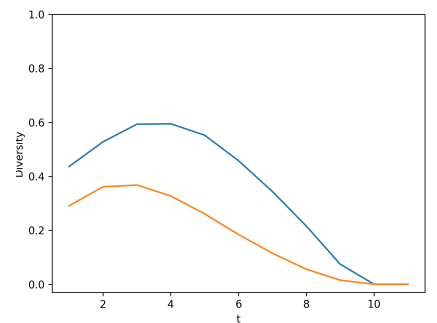

(a) $n_{U}=1, n_{Y^{*}}=1$

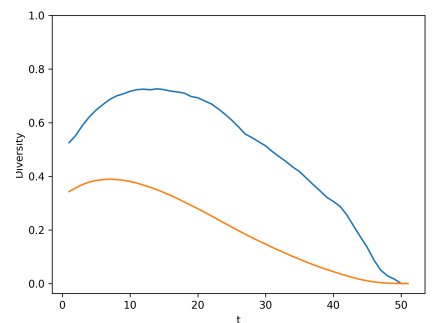

(d) $n_{U}=50, n_{Y^{*}}=1$

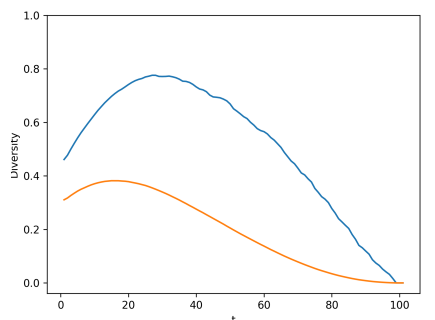

(g) $n_{U}=100, n_{Y^{*}}=1$

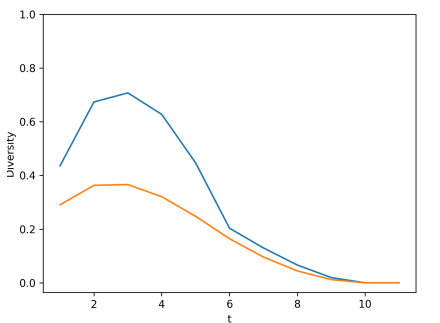

(b) $n_{U}=10, n_{Y^{*}}=5$

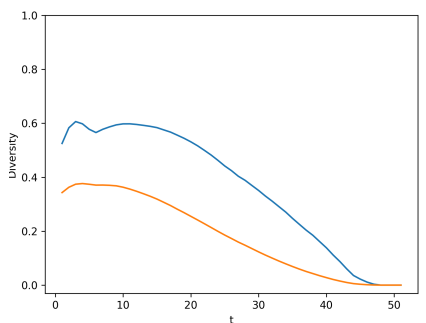

(e) $n_{U}=50, n_{Y^{*}}=5$

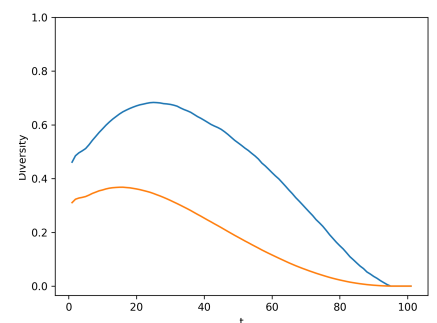

(h) $n_{U}=100, n_{Y^{*}}=5$

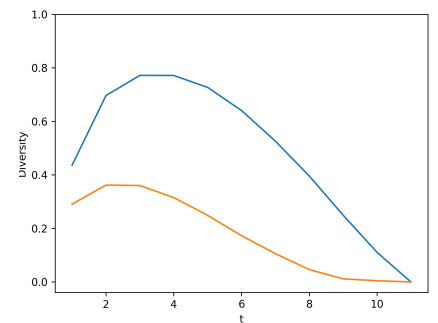

(c) $n_{U}=10, n_{Y^{*}}=10$

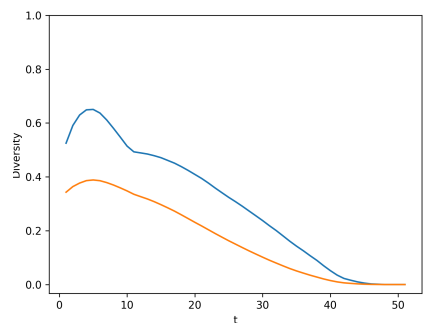

(f) $n_{U}=50, n_{Y^{*}}=10$

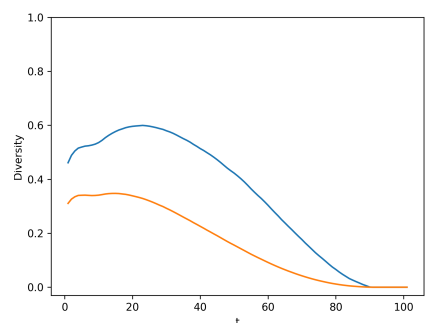

(i) $n_{U}=100, n_{Y^{*}}=10$

Figure 5.2: Jaccard Diversity (blue) and Hamming Diversity (orange) for Experiment 2

\subsection{Summary}

This chapter proposed two methods for calculating the diversity of set-based populations, namely Jaccard diversity and Hamming diversity based on their respective distance measures. It was shown that the diversity reflects the correct trends as expected as a population moves from maximum diversity to minimum diversity. Jaccard diversity resulted in big changes in diversity when small-sized sets were considered. Large, oscillating changes in Jaccard diversity can give the false impression that the population is switching back and forth between states of exploration and exploitation. Hamming distance produced more subtle changes in diversity. Hamming diversity regards that sets are similar in regard to the elements they commonly exclude. Thus, in cases where the elements of sets differ completely, Hamming diversity still regards these sets as similar. As a result of this similarity between completely different sets, the Hamming diversity is shown to under-represent diversity, especially in cases where diversity is intuitively at a maximum. Therefore, the 
author believes that Jaccard diversity better represents about set diversity. 


\section{Chapter 6}

\section{Set-based Particle Swarm Optimization Improvement and Diversity Analysis}

The focus of this chapter 1 is to address the gap in performance between SBPSO and the two top performing algorithms, namely ABC and PSO, as reported in Chapter 4. Several design problems of the proposed approach to portfolio optimization are identified, from which possible improvements are derived and investigated. Furthermore, the newly defined Jaccard diversity measure is used to better understand the exploration and exploitation behaviour of SBPSO.

The rest of this chapter is organized as follows: The design problems are discussed in Section 6.1, followed by an analysis of the Jaccard diversity values of SBPSO for portfolio optimization in Section 6.2. Modifications to improve the performance of SBPSO are given in Section 6.3. Section 6.4 proposes an alternative weight optimizer using ACD. Section 6.5 evaluates the performance between SBPSO with modifications and SBPSO and the other algorithms in Chapter 4. The findings are summarised in Section 6.6.

\subsection{Design Problems}

The bi-stage search process of SBPSO demonstrated good performance for portfolio optimization problems, better than the FA and comparable to that of the GA, but not as good as ABC and PSO. The advantage of SBPSO over the other algorithms was that it was significantly faster, and the search time was invariant to the number of assets considered, albeit at the cost of

\footnotetext{
${ }^{1}$ This chapter was published in the proceedings of the 2020 IEEE Swarm Intelligence Symposium under the title "Improved Set-based Particle Swarm optimization for Portfolio optimization" [54]. This chapter is also currently being reviewed for the IEEE Swarm Intelligence Symposium under the title "Set-based Particle Swarm Optimization for Portfolio Optimization with Adaptive Coordinate Descent Weight Optimization" [59].
} 
a slight degradation in solution quality. The degradation in performance is, in general, hypothesized to be a result of an inadequate weight determination process. The following outlines, in hindsight, the design problems of SBPSO:

- The weight-determination stage was limited to 10 iterations, which is not sufficient to finely tune the weights of a candidate solution. Furthermore, when a set-particle contains only one asset, such as in the case of strictly maximizing profit, the next 10 iterations are spent determining the asset's weight. However, considering equation (3.4), the weight of an asset in a single-asset portfolio can only ever have a value of one. Thus, 10 iterations would then be unnecessarily wasted and increase the time to reach the stopping condition.

- After the weight determination stage, it may be the case that the weights of certain assets are zero, meaning that they have no impact on the value of a portfolio. Yet, these assets remain in the position of a particle, making them likely to re-appear in the future. The existence of the zeroweighted assets unnecessarily increases the dimensionality in the weight determination stage and adds noise. Furthermore, the ability of SBPSO to converge to a single set-solution is also affected. For example, if the weight determination process for three candidate solutions, $\{5,1,2\},\{5$, $3,4\}$ and $\{5,6,7\}$, assigns a weight of one to asset 5 , while all other assets have a weight of zero, all candidate solutions will have the same objective function value. In light of this, the proposed SBPSO approach will converge to similar weights, but not necessarily a set of common elements.

\subsection{Diversity Analysis}

Figure 6.1 shows that the SBPSO for portfolio optimization is unable to converge to a single solution. The Jaccard diversity for SBPSO generally stagnates around $60 \%$ dissimilarity throughout the search, except for FTSE 100 where it dips to between $40 \%$ and $60 \%$. Nikkei 225 also shows a slight downwards trend, but quickly stagnates. Hence, SBPSO spends too much time exploring the search space and not enough refining solutions. The Jaccard diversity values in Figure 6.1 are used as a baseline which the proposed modifications are compared to. The proposed modifications are expected to balance the exploration and exploitation behavior of the SBPSO for portfolio optimization, while also improving solution quality. 


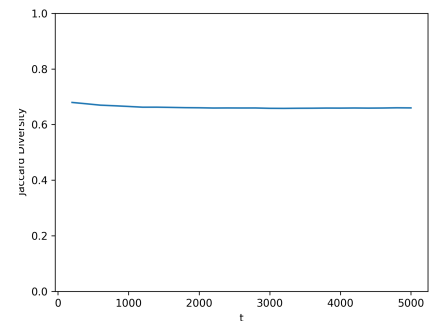

(a) Hang Seng

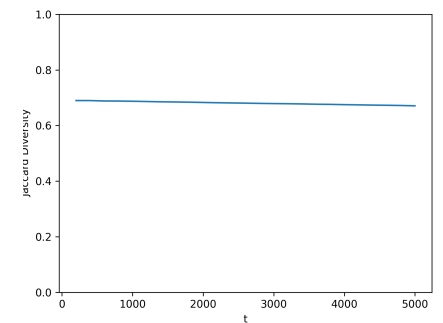

(b) DAX 100

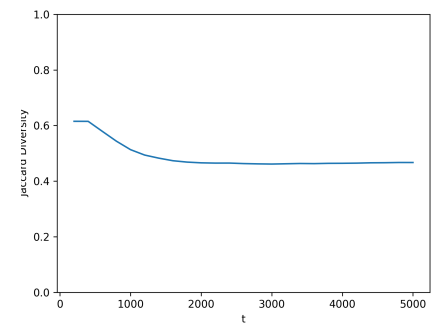

(c) FTSE 100

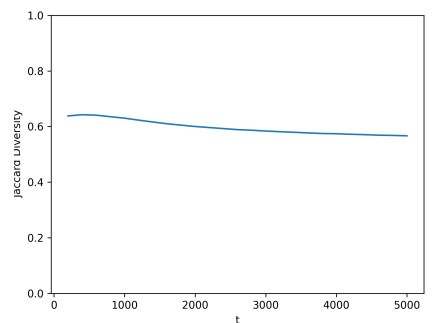

(d) S\&P 100

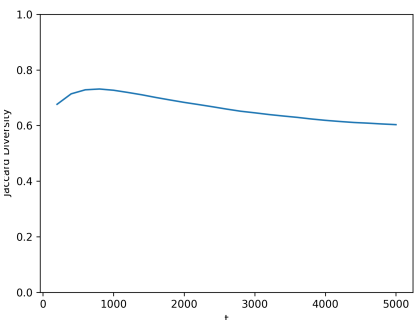

(e) Nikkei 225

Figure 6.1: Average Jaccard diversity over time for SBPSO

\subsection{Modifications}

This section iteratively suggests and investigates modifications of SBPSO, based on the aforementioned issues, to create a more problem-specific version for portfolio optimization by leveraging the relationship with the weight optimizer. Each modification builds upon the previous, unless the modification severely impacts performance. Each modified version of SBPSO, of which there are six, is referred to as $\mathrm{SBPSO}_{\mathrm{m} x}$, where $x$ is the corresponding modification number. For example, the first modified version of SBPSO is referred to as $\mathrm{SBPSO}_{\mathrm{m} 1}$, the second is $\mathrm{SBPSO}_{\mathrm{m} 2}$, and so on.

Section 6.3.1 proposes a modification to remove zero-weighted assets from the set-particles. A more efficient approach to optimizing single-asset portfolios is given in Section 6.3.2. Section 6.3.3 proposes a modification that allows the weight optimizer to execute until it converges. A modification to only allow assets to be removed from set-particle positions if the assets are zeroweighted is proposed in Section 6.3.4. Section 6.3.5 reintroduces tournament selection to the SBPSO velocity update equation. A modification to balance the exploration and exploitation behaviour is presented in Section 6.3.6.

\subsubsection{Removal of Zero-weighted Assets}

The first of the suggested modifications is quite simple. Any assets that have a weighting of zero after the weight determination process are removed from the position of the set-particle. Thus, set-positions become smaller and reduce the dimensionality of future PSO searches, thereby making it easier and faster for 
CHAPTER 6. SET-BASED PARTICLE SWARM OPTIMIZATION

IMPROVEMENT AND DIVERSITY ANALYSIS

the PSO to identify good-quality weights. It is expected that this modification will significantly decrease the time to obtain good solutions.

Figure 6.2 shows that the average Jaccard diversity of $\mathrm{SBPSO}_{\mathrm{m} 1}$ for all portfolio optimization problems was much higher than that of SBPSO. The particles in $\mathrm{SBPSO}_{\mathrm{m} 1}$ are $80 \%$ to $100 \%$ dissimilar, whereas the particles in SBPSO were $20 \%$ less dissimilar. Thus, the Jaccard diversity for $\mathrm{SBPSO}_{\mathrm{m} 1}$ was worse than SBPSO for portfolio optimization. However, this is not to say that the convergence of SBPSO was good, as both versions were shown to be in a constant state of exploration. The addition of this modification sheds light on the fact that the portfolios obtained by SBPSO contain common zeroweighted assets, leading to lower Jaccard diversity values. Table 6.1 shows the SBPSO set-particles contained a high number of common zero-weight assets for all benchmark problems.

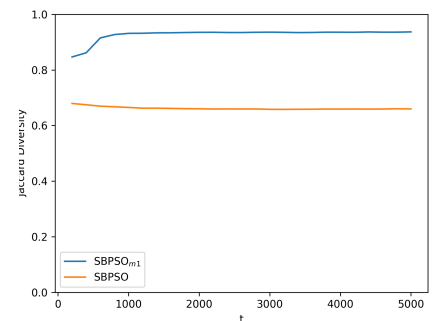

(a) Hang Seng

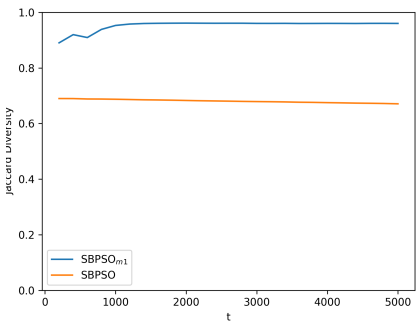

(b) DAX 100

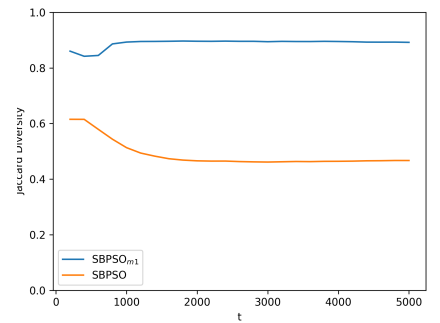

(c) FTSE 100

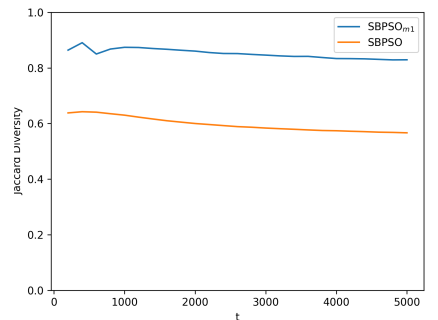

(d) $\mathrm{S} \& \mathrm{P} 100$

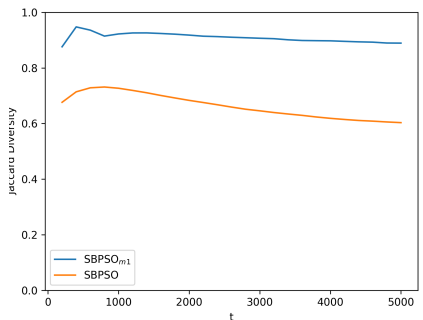

(e) Nikkei 225

Figure 6.2: Average Jaccard Diversity over Time for $\mathrm{SBPSO}_{\mathrm{m} 1}$ 
CHAPTER 6. SET-BASED PARTICLE SWARM OPTIMIZATION IMPROVEMENT AND DIVERSITY ANALYSIS

Table 6.1: $\mathrm{SBPSO}$ versus $\mathrm{SBPSO}_{\mathrm{m} 1}$ : Number of zero-weight assets for each benchmark problem

\begin{tabular}{|c|c|c|c|c|c|c|c|}
\hline & Algorithm & Result & $K_{\lambda=0.0}$ & $K_{\lambda=1.0}$ & $K$ & $K_{0}$ & $K_{0, \forall x_{i}}$ \\
\hline \multirow[t]{4}{*}{ Hang Seng } & SBPSO & $\bar{x}$ & 13.50 & 17.44 & 14.86 & 11.66 & 12.87 \\
\hline & & $\sigma$ & 1.68 & 1.69 & 2.27 & 1.65 & 1.95 \\
\hline & $\mathrm{SBPSO}_{\mathrm{m} 1}$ & $\bar{x}$ & 1.19 & 5.23 & 2.43 & 0.00 & 0.00 \\
\hline & & $\sigma$ & 0.14 & 0.33 & 1.36 & 0.00 & 0.00 \\
\hline \multirow[t]{4}{*}{ DAX 100} & SBPSO & $\bar{x}$ & 37.68 & 50.33 & 40.11 & 28.03 & 38.76 \\
\hline & & $\sigma$ & 5.44 & 4.42 & 5.70 & 3.03 & 5.49 \\
\hline & $\mathrm{SBPSO}_{\mathrm{m} 1}$ & $\bar{x}$ & 1.28 & 11.76 & 3.19 & 0.00 & 0.00 \\
\hline & & $\sigma$ & 0.24 & 0.64 & 2.72 & 0.00 & 0.00 \\
\hline \multirow[t]{4}{*}{ FTSE 100} & SBPSO & $\bar{x}$ & 23.97 & 42.06 & 26.83 & 19.63 & 23.97 \\
\hline & & $\sigma$ & 10.62 & 11.36 & 12.00 & 7.27 & 12.16 \\
\hline & $\mathrm{SBPSO}_{\mathrm{m} 1}$ & $\bar{x}$ & 1.02 & 11.14 & 2.82 & 0.00 & 0.00 \\
\hline & & $\sigma$ & 0.04 & 1.25 & 2.72 & 0.00 & 0.00 \\
\hline \multirow[t]{4}{*}{ S\&P 100} & SBPSO & $\bar{x}$ & 32.60 & 56.06 & 38.49 & 26.08 & 35.91 \\
\hline & & $\sigma$ & 12.36 & 10.27 & 13.54 & 6.99 & 13.74 \\
\hline & $\mathrm{SBPSO}_{\mathrm{m} 1}$ & $\bar{x}$ & 1.01 & 13.56 & 3.99 & 0.00 & 0.00 \\
\hline & & $\sigma$ & 0.02 & 0.89 & 3.91 & 0.00 & 0.00 \\
\hline \multirow[t]{4}{*}{ Nikkei 225} & SBPSO & $\bar{x}$ & 47.54 & 59.54 & 50.55 & 35.18 & 44.42 \\
\hline & & $\sigma$ & 17.75 & 21.33 & 18.37 & 10.54 & 18.44 \\
\hline & $\mathrm{SBPSO}_{\mathrm{m} 1}$ & $\bar{x}$ & 1.01 & 5.01 & 2.22 & 0.00 & 0.00 \\
\hline & & $\sigma$ & 0.02 & 0.34 & 1.19 & 0.00 & 0.00 \\
\hline
\end{tabular}

\footnotetext{
* For convenience, $K_{\lambda=0.0}$ is the size of the set-particle's position when optimizing the mean-variance model for return, $K_{\lambda=1.0}$ is the size of the set-particle's position when optimizing the mean-variance model for risk, $K$ is the average size of the set-particle's position, $K_{0}$ is the number of zero-weight assets in a set-particle's position, and $K_{0, \forall x_{i}}$ is the number of common zero-weighted assets across all set-particle positions. A common zero-weighted asset refers to a zero-weighted asset that appears in more than one set-particle position.
}

Although the modification to remove zero-weighted assets did not improve the diversity of the SBPSO, it did significantly improve its time to reach the stopping condition, as expected. Table 6.2 shows that the time results of $\mathrm{SBPSO}_{\mathrm{m} 1}$ for all benchmark problems was better than that of SBPSO. Performance gains can also be seen across other performance measures. Most notable, the size of the solutions obtained and the profitability of said solutions were statistically significantly better. Likewise, the GD, IGD and HV values were generally statistically significantly better. However, the riskiness of the portfolios obtained by $\mathrm{SBPSO}_{\mathrm{m} 1}$ for Hang Seng, DAX 100 and S\&P 100 was slightly worse than those obtained by SBPSO. Nevertheless, the gains in performance by simply removing zero-weighted assets from the set-particle's position are worthwhile and makes this modification a good first step towards improving the performance of SBPSO. 
CHAPTER 6. SET-BASED PARTICLE SWARM OPTIMIZATION IMPROVEMENT AND DIVERSITY ANALYSIS

Table 6.2: $\mathrm{SBPSO}$ versus $\mathrm{SBPSO}_{\mathrm{m} 1}$ : Mean and standard deviations for each performance measure

\begin{tabular}{|c|c|c|c|c|c|c|c|c|c|c|}
\hline & Algorithm & Result & $\mathbf{N}$ & $K$ & $R$ & $\bar{\sigma}$ & GD & IGD & HV & Time \\
\hline \multirow[t]{4}{*}{ Hang Seng } & SBPSO & $\bar{x}$ & 34 & 3 & 0.007614 & 0.001908 & 0.000463 & 0.000236 & 0.776926 & 9.37 \\
\hline & & $\sigma$ & 1 & 0 & 0.000022 & 0.000009 & 0.000141 & 0.000010 & 0.023747 & 0.53 \\
\hline & $\mathrm{SBPSO}_{\mathrm{m} 1}$ & $\bar{x}$ & 33 & 3 & 0.007629 & 0.001937 & 0.000816 & 0.000253 & 0.768025 & 4.70 \\
\hline & & $\sigma$ & 1 & 0 & 0.000071 & 0.000047 & 0.000133 & 0.000014 & 0.027005 & 0.41 \\
\hline \multirow[t]{4}{*}{ DAX 100} & SBPSO & $\bar{x}$ & 34 & 6 & 0.007670 & 0.000960 & 0.002334 & 0.000357 & 0.826267 & 26.87 \\
\hline & & $\sigma$ & 5 & 1 & 0.000319 & 0.000105 & 0.000527 & 0.000019 & 0.054478 & 1.63 \\
\hline & $\mathrm{SBPSO}_{\mathrm{m} 1}$ & $\bar{x}$ & 38 & 3 & 0.008359 & 0.001013 & 0.001258 & 0.000358 & 0.840054 & 6.15 \\
\hline & & $\sigma$ & 3 & 0 & 0.000105 & 0.000056 & 0.000402 & 0.000026 & 0.064622 & 0.91 \\
\hline \multirow[t]{4}{*}{ FTSE 100} & SBPSO & $\bar{x}$ & 33 & 6 & 0.006605 & 0.000822 & 0.001206 & 0.000264 & 0.725351 & 13.81 \\
\hline & & $\sigma$ & 6 & 2 & 0.000176 & 0.000061 & 0.000472 & 0.000017 & 0.037266 & 5.43 \\
\hline & $\mathrm{SBPSO}_{\mathrm{m} 1}$ & $\bar{x}$ & 29 & 3 & 0.006693 & 0.000797 & 0.000596 & 0.000247 & 0.738303 & 6.13 \\
\hline & & $\sigma$ & 1 & 0 & 0.000036 & 0.000018 & 0.000163 & 0.000010 & 0.035322 & 1.17 \\
\hline \multirow[t]{4}{*}{ S\&P 100} & SBPSO & $\bar{x}$ & 43 & 9 & 0.007269 & 0.001219 & 0.001775 & 0.000301 & 0.890120 & 35.69 \\
\hline & & $\sigma$ & 2 & 3 & 0.000428 & 0.000174 & 0.000620 & 0.000036 & 0.031804 & 5.56 \\
\hline & $\mathrm{SBPSO}_{\mathrm{m} 1}$ & $\bar{x}$ & 44 & 5 & 0.007746 & 0.001331 & 0.000690 & 0.000272 & 0.875669 & 7.34 \\
\hline & & $\sigma$ & 1 & 0 & 0.000037 & 0.000027 & 0.000096 & 0.000012 & 0.039881 & 1.86 \\
\hline \multirow[t]{4}{*}{ Nikkei 225} & SBPSO & $\bar{x}$ & 42 & 10 & 0.002454 & 0.000800 & 0.002396 & 0.000353 & 0.826649 & 29.22 \\
\hline & & $\sigma$ & 4 & 5 & 0.000592 & 0.000065 & 0.000535 & 0.000065 & 0.067572 & 4.19 \\
\hline & $\mathrm{SBPSO}_{\mathrm{m} 1}$ & $\bar{x}$ & 41 & 3 & 0.003264 & 0.000792 & 0.000788 & 0.000264 & 0.911819 & 8.55 \\
\hline & & $\sigma$ & 2 & 0 & 0.000033 & 0.000022 & 0.000101 & 0.000019 & 0.051362 & 0.40 \\
\hline
\end{tabular}

\subsubsection{Single Asset Portfolios}

The second proposed modification addresses the needless optimization of single asset portfolios. To reiterate, SBPSO uses 10 iterations to optimize the weight of a single asset portfolio. Given equation (3.4), the weight can only have a value of 1.0. Thus, the second modification is to assign a contribution weight of 1.0 if the position of a set-particle contains exactly one asset, and to then immediately calculate the objective function. Thus, no weight determination process is required. It is expected that this modification will not impact the Pareto results obtained, but rather decrease the time to reach the stopping condition.

Table 6.3 shows that performance of the $\mathrm{SBPSO}_{\mathrm{m} 1}$ and $\mathrm{SBPSO}_{\mathrm{m} 2}$ is identical. As expected, the search time of $\mathrm{SBPSO}_{\mathrm{m} 2}$ was slightly better than that of $\mathrm{SBPSO}_{\mathrm{m} 1}$. It should be noted that the algorithms used the same set of seeds for random number generation which is why the results (other than time) are identical. The average Jaccard diversity for $\mathrm{SBPSO}_{\mathrm{m} 2}$, shown in Figure 6.3, is also identical to that of $\mathrm{SBPSO}_{\mathrm{m} 1}$ in Figure 6.2. Nevertheless, it is a modification that provides good housekeeping, so to speak, at no cost to performance. 
CHAPTER 6. SET-BASED PARTICLE SWARM OPTIMIZATION IMPROVEMENT AND DIVERSITY ANALYSIS

Table 6.3: $\mathrm{SBPSO}_{\mathrm{m} 1}$ versus $\mathrm{SBPSO}_{\mathrm{m} 2}$ : Mean and standard deviations for each performance measure

\begin{tabular}{|c|c|c|c|c|c|c|c|c|c|c|}
\hline & Algorithm & Result & $\mathbf{N}$ & $K$ & $R$ & $\bar{\sigma}$ & GD & IGD & HV & Time \\
\hline \multirow[t]{4}{*}{ Hang Seng } & $\mathrm{SBPSO}_{\mathrm{m} 1}$ & $\bar{x}$ & 33 & 3 & 0.007629 & 0.001937 & 0.000816 & 0.000253 & 0.768025 & 4.70 \\
\hline & & $\sigma$ & 1 & 0 & 0.000071 & 0.000047 & 0.000133 & 0.000014 & 0.027005 & 0.41 \\
\hline & $\mathrm{SBPSO}_{\mathrm{m} 2}$ & $\bar{x}$ & 33 & 3 & 0.007629 & 0.001937 & 0.000816 & 0.000253 & 0.768025 & 4.13 \\
\hline & & $\sigma$ & 1 & 0 & 0.000071 & 0.000047 & 0.000133 & 0.000014 & 0.027005 & 0.65 \\
\hline \multirow[t]{4}{*}{ DAX 100} & $\mathrm{SBPSO}_{\mathrm{m} 1}$ & $\bar{x}$ & 38 & 3 & 0.008359 & 0.001013 & 0.001258 & 0.000358 & 0.840054 & 6.15 \\
\hline & & $\sigma$ & 3 & 0 & 0.000105 & 0.000056 & 0.000402 & 0.000026 & 0.064622 & 0.91 \\
\hline & $\mathrm{SBPSO}_{\mathrm{m} 2}$ & $\bar{x}$ & 38 & 3 & 0.008359 & 0.001013 & 0.001258 & 0.000358 & 0.840054 & 5.94 \\
\hline & & $\sigma$ & 3 & 0 & 0.000105 & 0.000056 & 0.000402 & 0.000026 & 0.064622 & 1.09 \\
\hline \multirow[t]{4}{*}{ FTSE 100} & $\mathrm{SBPSO}_{\mathrm{m} 1}$ & $\bar{x}$ & 29 & 3 & 0.006693 & 0.000797 & 0.000596 & 0.000247 & 0.738303 & 6.13 \\
\hline & & $\sigma$ & 1 & 0 & 0.000036 & 0.000018 & 0.000163 & 0.000010 & 0.035322 & 1.17 \\
\hline & $\mathrm{SBPSO}_{\mathrm{m} 2}$ & $\bar{x}$ & 29 & 3 & 0.006693 & 0.000797 & 0.000596 & 0.000247 & 0.738303 & 5.29 \\
\hline & & $\sigma$ & 1 & 0 & 0.000036 & 0.000018 & 0.000163 & 0.000010 & 0.035322 & 1.66 \\
\hline \multirow[t]{4}{*}{ S\&P 100} & $\mathrm{SBPSO}_{\mathrm{m} 1}$ & $\bar{x}$ & 44 & 5 & 0.007746 & 0.001331 & 0.000690 & 0.000272 & 0.875669 & 7.34 \\
\hline & & $\sigma$ & 1 & 0 & 0.000037 & 0.000027 & 0.000096 & 0.000012 & 0.039881 & 1.86 \\
\hline & $\mathrm{SBPSO}_{\mathrm{m} 2}$ & $\bar{x}$ & 44 & 5 & 0.007746 & 0.001331 & 0.000690 & 0.000272 & 0.875669 & 7.13 \\
\hline & & $\sigma$ & 1 & 0 & 0.000037 & 0.000027 & 0.000096 & 0.000012 & 0.039881 & 1.88 \\
\hline \multirow[t]{4}{*}{ Nikkei 225} & $\mathrm{SBPSO}_{\mathrm{m} 1}$ & $\bar{x}$ & 41 & 3 & 0.003264 & 0.000792 & 0.000788 & 0.000264 & 0.911819 & 8.55 \\
\hline & & $\sigma$ & 2 & 0 & 0.000033 & 0.000022 & 0.000101 & 0.000019 & 0.051362 & 0.40 \\
\hline & $\mathrm{SBPSO}_{Z} S$ & $\bar{x}$ & 41 & 3 & 0.003264 & 0.000792 & 0.000788 & 0.000264 & 0.911819 & 7.78 \\
\hline & & $\sigma$ & 2 & 0 & 0.000033 & 0.000022 & 0.000101 & 0.000019 & 0.051362 & 0.62 \\
\hline
\end{tabular}

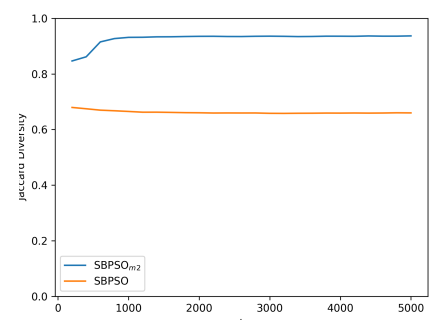

(a) Hang Seng

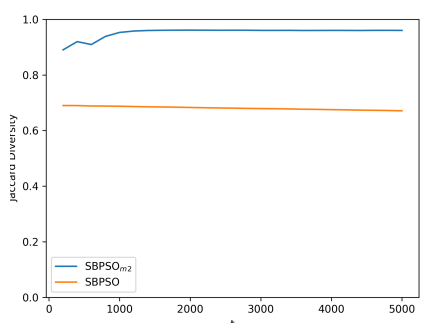

(b) DAX 100

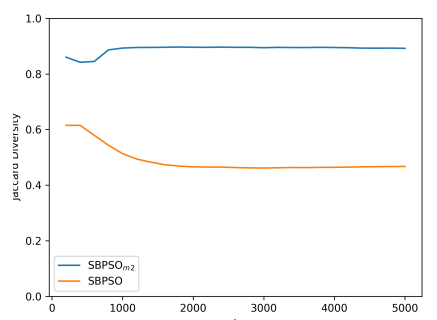

(c) FTSE 100

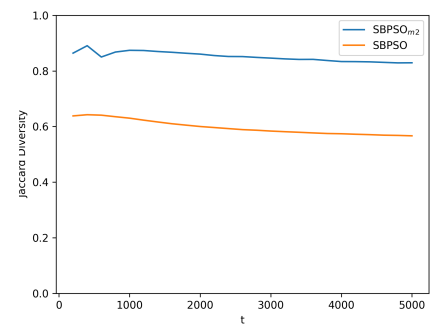

(d) S\&P 100

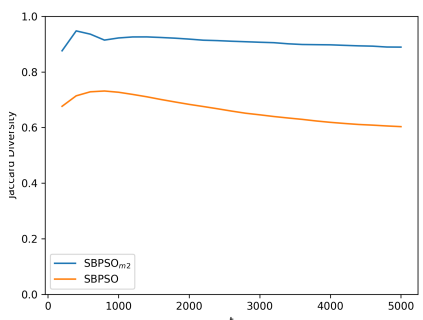

(e) Nikkei 225

Figure 6.3: Average Jaccard Diversity over Time for $\mathrm{SBPSO}_{\mathrm{m} 2}$

\subsubsection{Weight Determination Until Convergence}

Instead of allocating 10 iterations to the weight optimization of assets, this modification allows the weight optimizer to execute until there is no improve- 
ment in solution quality. Solution quality is measured as the best objective function value. If solution quality does not improve over three iterations, the weight optimization process stops. A limit of three iterations is chosen instead of larger limit to avoid too much time spent optimizing the weights and maintain the fast search time. Furthermore, a limit smaller than three could end the weight determination process too early and forsake potential performance gains. If the stopping condition is reached during the weight optimization process, then the weight optimizer stops and the SBPSO search terminates. It is expected that this modification will improve the Pareto-optimality related results because the weight optimizer has more time to refine solutions.

Table 6.4 shows that the average number of solutions obtained and profitability of $\mathrm{SBPSO}_{\mathrm{m} 3}$ was greater than of $\mathrm{SBPSO}_{\mathrm{m} 2}$. Likewise, the average GD, IGD and HV scores were better with lower standard deviations. In other words, the performance of $\mathrm{SBPSO}_{\mathrm{m} 3}$ was better and more consistent. The increases in performance were verified by statistical significance tests that show for the aforementioned performances measures, that the performance of $\mathrm{SBPSO}_{\mathrm{m}}$ was generally better or the same as $\mathrm{SBPSO}_{\mathrm{m} 2} \cdot \mathrm{SBPSO}_{\mathrm{m} 3}$ was slower to reach the stopping condition than $\mathrm{SBPSO}_{\mathrm{m} 2}$, but still better than or comparable to SBPSO.

Table 6.4: $\mathrm{SBPSO}_{\mathrm{m} 2}$ versus $\mathrm{SBPSO}_{\mathrm{m} 3}$ : Mean and standard deviations for each performance measure

\begin{tabular}{|c|c|c|c|c|c|c|c|c|c|c|}
\hline & Algorithm & Result & $\mathbf{N}$ & $K$ & $R$ & $\bar{\sigma}$ & GD & IGD & HV & Time \\
\hline \multirow[t]{4}{*}{ Hang Seng } & \multirow{2}{*}{$\mathrm{SBPSO}_{\mathrm{m} 2}$} & $\bar{x}$ & 33 & 3 & 0.007637 & 0.001921 & 0.000590 & 0.000241 & 0.767049 & 5.33 \\
\hline & & $\sigma$ & 0 & 0 & 0.000035 & 0.000024 & 0.000183 & 0.000011 & 0.028889 & 1.25 \\
\hline & \multirow[t]{2}{*}{$\mathrm{SBPSO}_{\mathrm{m} 3}$} & $\bar{x}$ & 33 & 3 & 0.007614 & 0.001900 & 0.000274 & 0.000217 & 0.785971 & 7.04 \\
\hline & & $\sigma$ & 1 & 0 & 0.000007 & 0.000005 & 0.000088 & 0.000006 & 0.019312 & 1.89 \\
\hline \multirow[t]{4}{*}{ DAX 100} & \multirow[t]{2}{*}{$\mathrm{SBPSO}_{\mathrm{m} 2}$} & $\bar{x}$ & 42 & 4 & 0.008487 & 0.000997 & 0.000506 & 0.000328 & 0.850058 & 10.87 \\
\hline & & $\sigma$ & 2 & 0 & 0.000056 & 0.000027 & 0.000250 & 0.000021 & 0.062547 & 2.47 \\
\hline & \multirow[t]{2}{*}{$\mathrm{SBPSO}_{\mathrm{m} 3}$} & $\bar{x}$ & 43 & 4 & 0.008498 & 0.000986 & 0.000413 & 0.000330 & 0.851289 & 12.60 \\
\hline & & $\sigma$ & 1 & 0 & 0.000020 & 0.000013 & 0.000119 & 0.000017 & 0.068197 & 2.69 \\
\hline \multirow[t]{4}{*}{ FTSE 100} & \multirow[t]{2}{*}{$\mathrm{SBPSO}_{\mathrm{m} 2}$} & $\bar{x}$ & 30 & 4 & 0.006696 & 0.000795 & 0.000433 & 0.000240 & 0.747358 & 14.97 \\
\hline & & $\sigma$ & 1 & 0 & 0.000021 & 0.000007 & 0.000090 & 0.000007 & 0.035120 & 3.80 \\
\hline & \multirow[t]{2}{*}{$\mathrm{SBPSO}_{\mathrm{m} 3}$} & $\bar{x}$ & 30 & 4 & 0.006684 & 0.000786 & 0.000282 & 0.000237 & 0.745490 & 16.97 \\
\hline & & $\sigma$ & 0 & 0 & 0.000007 & 0.000001 & 0.000050 & 0.000006 & 0.045856 & 3.14 \\
\hline \multirow[t]{4}{*}{ S\&P 100} & \multirow[t]{2}{*}{$\mathrm{SBPSO}_{\mathrm{m} 2}$} & $\bar{x}$ & 44 & 5 & 0.007745 & 0.001291 & 0.000510 & 0.000260 & 0.877128 & 16.94 \\
\hline & & $\sigma$ & 0 & 0 & 0.000016 & 0.000032 & 0.000122 & 0.000008 & 0.038541 & 3.21 \\
\hline & \multirow[t]{2}{*}{$\mathrm{SBPSO}_{\mathrm{m} 3}$} & $\bar{x}$ & 44 & 6 & 0.007736 & 0.001275 & 0.000361 & 0.000253 & 0.865971 & 19.44 \\
\hline & & $\sigma$ & 0 & 0 & 0.000013 & 0.000023 & 0.000069 & 0.000008 & 0.041210 & 3.15 \\
\hline \multirow[t]{4}{*}{ Nikkei 225} & \multirow[t]{2}{*}{$\mathrm{SBPSO}_{\mathrm{m} 2}$} & $\bar{x}$ & 43 & 3 & 0.003299 & 0.000794 & 0.000478 & 0.000240 & 0.919549 & 41.38 \\
\hline & & $\sigma$ & 2 & 0 & 0.000034 & 0.000024 & 0.000141 & 0.000015 & 0.048358 & 3.20 \\
\hline & \multirow[t]{2}{*}{$\mathrm{SBPSO}_{\mathrm{m} 3}$} & $\bar{x}$ & 44 & 4 & 0.003305 & 0.000790 & 0.000323 & 0.000230 & 0.908527 & 49.23 \\
\hline & & $\sigma$ & 1 & 0 & 0.000020 & 0.000017 & 0.000080 & 0.000008 & 0.036250 & 5.68 \\
\hline
\end{tabular}

Unfortunately, the exploitation behaviour of $\mathrm{SBPSO}_{\mathrm{m} 3}$ was no better than that of $\mathrm{SBPSO}_{\mathrm{m} 2}$, as seen in Figure 6.4. This suggests that the imbalance in the exploratory and exploitative behaviour is not a result of the weight 
determination stage, but rather the formulation of SBPSO for portfolio optimization. Even so, allowing the weight determination to execute until there is no improvement in solution quality, yields significant gains in other performance metrics.

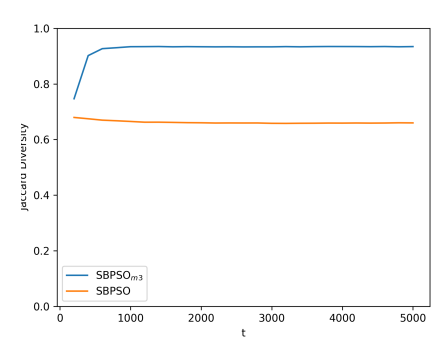

(a) Hang Seng

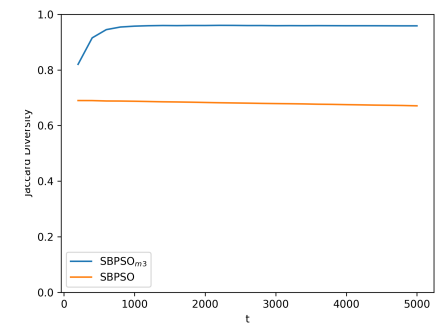

(b) DAX 100

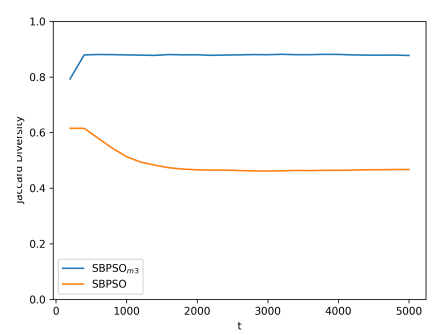

(c) FTSE 100

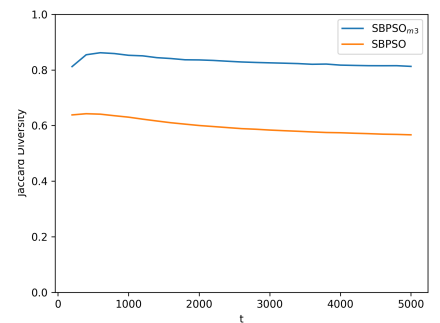

(d) S\&P 100

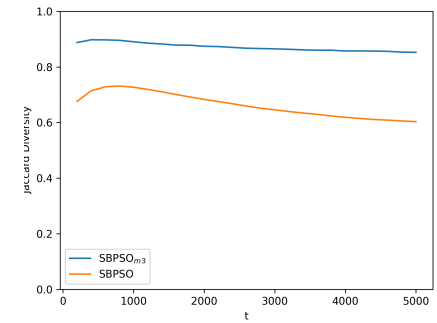

(e) Nikkei 225

Figure 6.4: Average Jaccard Diversity over Time for $\mathrm{SBPSO}_{\mathrm{m} 3}$

\subsubsection{Asset Removal}

This modification investigates the impact of the $\odot^{-}$operator on the performance of SBPSO for portfolio optimization. The $\odot^{-}$operator is responsible for removing elements in $X(t) \cup Y(t) \cup \hat{Y}(t)$ from $X(t)$. It is hypothesized that the $\odot^{-}$operator removes good-quality assets that would otherwise be useful in the weight determination stage. This modification excludes the $\odot^{-}$operator from the velocity update function. Therefore, assets can only be removed via the weight determination process, provided that the asset has a weighting of zero. This is arguably a more intuitive approach to asset removal, because it uses the information gained via weight determination to make intelligent decisions. The updated velocity equation is

$$
\begin{aligned}
V_{i}(t+1)= & c_{1} r_{1} \otimes\left(Y_{i}(t) \ominus X_{i}(t)\right) \\
& \oplus c_{2} r_{2} \otimes\left(\hat{Y}_{i}(t) \ominus X_{i}(t)\right) \\
& \oplus\left(c_{3} r_{3} \odot_{k}^{+} A_{i}(t)\right)
\end{aligned}
$$

It is expected that this modification will improve the convergence behaviour of SBPSO, because the set-particles will, over time, become more similar due to fewer assets being removed. It is also expected that solution quality will improve as a result of set-particles retaining good-quality assets. 
Figure 6.5 shows that $\mathrm{SBPSO}_{\mathrm{m} 4}$ was, in some cases, better at reducing diversity over time with reference to the Jaccard diversity values of SBPSO. FTSE 100 shows a smooth downwards trend from $80 \%$ to $20 \%$ dissimilarity. In other words, the set-particles were approximately $60 \%$ more similar at the end of the search than at the start. Other benchmarks show a decrease from $80 \%$ to $60 \%$ dissimilarity over time, similar to that of SBPSO. However, for DAX 100, the average Jaccard diversity was worse for $\mathrm{SBPSO}_{\mathrm{m} 4}$ at about $80 \%$ dissimilarity throughout the search.

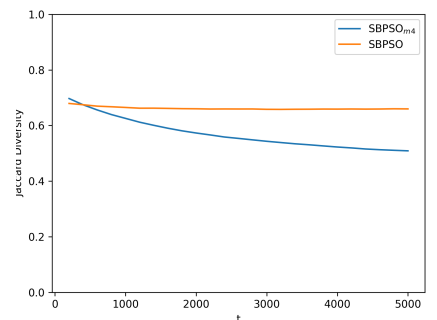

(a) Hang Seng

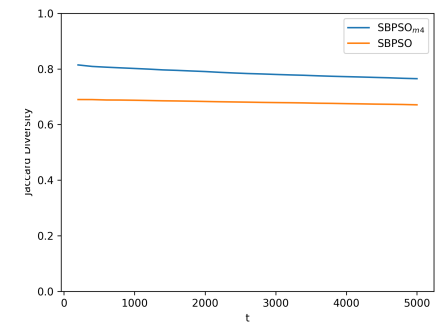

(b) DAX 100

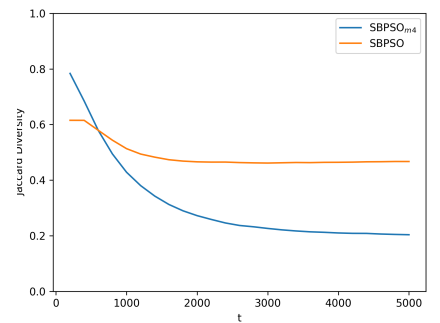

(c) FTSE 100

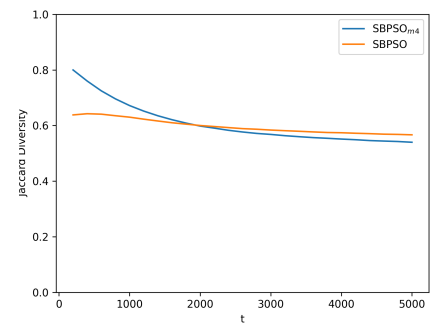

(d) S\&P 100

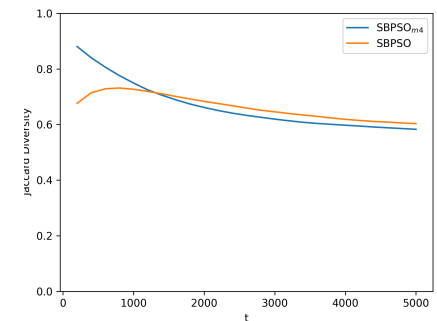

(e) Nikkei 225

Figure 6.5: Average Jaccard Diversity over Time for $\mathrm{SBPSO}_{\mathrm{m}}$

The performance of $\mathrm{SBPSO}_{\mathrm{m} 4}$ was very similar to that of $\mathrm{SBPSO}_{\mathrm{m} 3}$, as seen in Table 6.5. However, the standard deviation of the performance scores for $\mathrm{SBPSO}_{\mathrm{m}}$ was typically half of that of $\mathrm{SBPSO}_{\mathrm{m} 3}$, and in some cases better by an order of magnitude. Statistical significance tests also show that the GD, IGD and HV scores of $\mathrm{SBPSO}_{\mathrm{m} 4}$ were the same or better than those of $\mathrm{SBPSO}_{\mathrm{m} 3}$ for all benchmark problems. Furthermore, the risk values of $\mathrm{SBPSO}_{\mathrm{m}}$ were lower than those of $\mathrm{SBPSO}_{\mathrm{m} 3}$. This modification is worth preserving, since it improved the results obtained and slightly improved the Jaccard diversity values of SBPSO. 
CHAPTER 6. SET-BASED PARTICLE SWARM OPTIMIZATION IMPROVEMENT AND DIVERSITY ANALYSIS

Table 6.5: $\mathrm{SBPSO}_{\mathrm{m} 3}$ versus $\mathrm{SBPSO}_{\mathrm{m} 4}$ : Mean and standard deviations for each performance measure

\begin{tabular}{|c|c|c|c|c|c|c|c|c|c|c|}
\hline & Algorithm & Result & $\mathbf{N}$ & $K$ & $R$ & $\bar{\sigma}$ & GD & IGD & HV & Time \\
\hline \multirow[t]{4}{*}{ Hang Seng } & \multirow[t]{2}{*}{$\mathrm{SBPSO}_{\mathrm{m} 3}$} & $\bar{x}$ & 33 & 3 & 0.007637 & 0.001921 & 0.000590 & 0.000241 & 0.767049 & 5.33 \\
\hline & & $\sigma$ & 0 & 0 & 0.000035 & 0.000024 & 0.000183 & 0.000011 & 0.028889 & 1.25 \\
\hline & \multirow[t]{2}{*}{$\mathrm{SBPSO}_{\mathrm{m} 4}$} & $\bar{x}$ & 33 & 3 & 0.007614 & 0.001900 & 0.000274 & 0.000217 & 0.785971 & 7.04 \\
\hline & & $\sigma$ & 1 & 0 & 0.000007 & 0.000005 & 0.000088 & 0.000006 & 0.019312 & 1.89 \\
\hline \multirow[t]{4}{*}{ DAX 100} & \multirow[t]{2}{*}{$\mathrm{SBPSO}_{\mathrm{m} 3}$} & $\bar{x}$ & 42 & 4 & 0.008487 & 0.000997 & 0.000506 & 0.000328 & 0.850058 & 10.87 \\
\hline & & $\sigma$ & 2 & 0 & 0.000056 & 0.000027 & 0.000250 & 0.000021 & 0.062547 & 2.47 \\
\hline & \multirow[t]{2}{*}{$\mathrm{SBPSO}_{\mathrm{m} 4}$} & $\bar{x}$ & 43 & 4 & 0.008498 & 0.000986 & 0.000413 & 0.000330 & 0.851289 & 12.60 \\
\hline & & $\sigma$ & 1 & 0 & 0.000020 & 0.000013 & 0.000119 & 0.000017 & 0.068197 & 2.69 \\
\hline \multirow[t]{4}{*}{ FTSE 100} & \multirow[t]{2}{*}{$\mathrm{SBPSO}_{\mathrm{m} 3}$} & $\bar{x}$ & 30 & 4 & 0.006696 & 0.000795 & 0.000433 & 0.000240 & 0.747358 & 14.97 \\
\hline & & $\sigma$ & 1 & 0 & 0.000021 & 0.000007 & 0.000090 & 0.000007 & 0.035120 & 3.80 \\
\hline & \multirow[t]{2}{*}{$\mathrm{SBPSO}_{\mathrm{m} 4}$} & $\bar{x}$ & 30 & 4 & 0.006684 & 0.000786 & 0.000282 & 0.000237 & 0.745490 & 16.97 \\
\hline & & $\sigma$ & 0 & 0 & 0.000007 & 0.000001 & 0.000050 & 0.000006 & 0.045856 & 3.14 \\
\hline \multirow[t]{4}{*}{ S\&P 100} & \multirow[t]{2}{*}{$\mathrm{SBPSO}_{\mathrm{m} 3}$} & $\bar{x}$ & 44 & 5 & 0.007745 & 0.001291 & 0.000510 & 0.000260 & 0.877128 & 16.94 \\
\hline & & $\sigma$ & 0 & 0 & 0.000016 & 0.000032 & 0.000122 & 0.000008 & 0.038541 & 3.21 \\
\hline & \multirow[t]{2}{*}{$\mathrm{SBPSO}_{\mathrm{m} 4}$} & $\bar{x}$ & 44 & 6 & 0.007736 & 0.001275 & 0.000361 & 0.000253 & 0.865971 & 19.44 \\
\hline & & $\sigma$ & 0 & 0 & 0.000013 & 0.000023 & 0.000069 & 0.000008 & 0.041210 & 3.15 \\
\hline \multirow[t]{4}{*}{ Nikkei 225} & \multirow[t]{2}{*}{$\mathrm{SBPSO}_{\mathrm{m} 3}$} & $\bar{x}$ & 43 & 3 & 0.003299 & 0.000794 & 0.000478 & 0.000240 & 0.919549 & 41.38 \\
\hline & & $\sigma$ & 2 & 0 & 0.000034 & 0.000024 & 0.000141 & 0.000015 & 0.048358 & 3.20 \\
\hline & \multirow[t]{2}{*}{$\mathrm{SBPSO}_{\mathrm{m} 4}$} & $\bar{x}$ & 44 & 4 & 0.003305 & 0.000790 & 0.000323 & 0.000230 & 0.908527 & 49.23 \\
\hline & & $\sigma$ & 1 & 0 & 0.000020 & 0.000017 & 0.000080 & 0.000008 & 0.036250 & 5.68 \\
\hline
\end{tabular}

\subsubsection{Tournament Selection}

The original SBPSO proposed by Langeveld and Engelbrecht implemented the $\odot^{+}$operator (refer to Section 2.4 using tournament selection. The purpose of the $\odot^{+}$operator is to add good quality elements to the positions of setparticles and to increase diversity. The SBPSO for portfolio optimization proposed in Chapter 4 redesigned the $\odot^{+}$operator to randomly select elements from $A$. This redesign was motivated by the removal of the selection bias of the tournament selection and to reduce computational complexity, since a weight determination process would be required for each element in the tournament. However, this section reintroduces tournament selection for the $\odot^{+}$operator as a means to better control the diversity added to set-particles and to calculate its impact on performance. It is expected that set-particles will become more similar as good quality assets are introduced to the set-particle positions by tournament selection. Furthermore, larger tournament sizes should result in less diversity since competitive assets would be frequently selected. To test this modification, tournament sizes $(\tau)$ of three, five and ten were used.

Table 6.6 shows that across all performance measures, except for time, the values obtained by $\mathrm{SBPSO}_{\mathrm{m} 4}$ and $\mathrm{SBPSO}_{\mathrm{m} 5}$ were mostly similar, despite the different tournament sizes. Furthermore, statistical significance tests that show that there was no difference in the size, profitability and riskiness of the obtained portfolios. $\mathrm{SBPSO}_{\mathrm{m} 5}$ was statistically significantly slower than $\mathrm{SBPSO}_{\mathrm{m} 4}$ for all tournament sizes. Furthermore, the time to reach the stop- 
ping condition worsens as the tournament size increases. The degradation in the time to reach the stopping condition was due to the weight determination required to calculate the objective function value for each asset in the tournament selection.

Table 6.6: $\mathrm{SBPSO}_{\mathrm{m} 4}$ versus $\mathrm{SBPSO}_{\mathrm{m} 5}$ : Mean and standard deviations for each performance measure

\begin{tabular}{|c|c|c|c|c|c|c|c|c|c|c|}
\hline & Algorithm & Result & $\mathbf{N}$ & $K$ & $R$ & $\bar{\sigma}$ & GD & IGD & HV & Time \\
\hline \multirow[t]{8}{*}{ Hang Seng } & \multirow[t]{2}{*}{$\mathrm{SBPSO}_{\mathrm{m} 4}$} & $\bar{x}$ & 33 & 3 & 0.007614 & 0.001900 & 0.000274 & 0.000217 & 0.785971 & 7.04 \\
\hline & & $\sigma$ & 1 & 0 & 0.000007 & 0.000005 & 0.000088 & 0.000006 & 0.019312 & 1.89 \\
\hline & \multirow{2}{*}{$\mathrm{SBPSO}_{\mathrm{m} 5, \tau=3}$} & $\bar{x}$ & 33 & 3 & 0.007614 & 0.001900 & 0.000333 & 0.000219 & 0.779533 & 24.33 \\
\hline & & $\sigma$ & 0 & 0 & 0.000010 & 0.000003 & 0.000110 & 0.000007 & 0.019819 & 13.83 \\
\hline & \multirow{2}{*}{$\mathrm{SBPSO}_{\mathrm{m} 5, \tau=5}$} & $\bar{x}$ & 34 & 3 & 0.007614 & 0.001900 & 0.000289 & 0.000216 & 0.791427 & 38.70 \\
\hline & & $\sigma$ & 0 & 0 & 0.000009 & 0.000006 & 0.000092 & 0.000006 & 0.018159 & 22.35 \\
\hline & \multirow{2}{*}{$\mathrm{SBPSO}_{\mathrm{m} 5, \tau=10}$} & $\bar{x}$ & 34 & 3 & 0.007614 & 0.001900 & 0.000272 & 0.000216 & 0.792602 & 71.37 \\
\hline & & $\sigma$ & 0 & 0 & 0.000006 & 0.000002 & 0.000094 & 0.000005 & 0.019566 & 47.08 \\
\hline \multirow[t]{8}{*}{ DAX 100} & \multirow{2}{*}{$\mathrm{SBPSO}_{\mathrm{m} 4}$} & $\bar{x}$ & 43 & 4 & 0.008498 & 0.000986 & 0.000413 & 0.000330 & 0.851289 & 12.60 \\
\hline & & $\sigma$ & 1 & 0 & 0.000020 & 0.000013 & 0.000119 & 0.000017 & 0.068197 & 2.69 \\
\hline & \multirow{2}{*}{$\mathrm{SBPSO}_{\mathrm{m} 5, \tau=3}$} & $\bar{x}$ & 43 & 4 & 0.008497 & 0.000986 & 0.000386 & 0.000322 & 0.853886 & 70.29 \\
\hline & & $\sigma$ & 1 & 0 & 0.000019 & 0.000012 & 0.000124 & 0.000017 & 0.063812 & 19.43 \\
\hline & \multirow{2}{*}{$\mathrm{SBPSO}_{\mathrm{m} 5, \tau=5}$} & $\bar{x}$ & 42 & 4 & 0.008493 & 0.000980 & 0.000384 & 0.000325 & 0.850306 & 82.99 \\
\hline & & $\sigma$ & 1 & 0 & 0.000016 & 0.000007 & 0.000101 & 0.000013 & 0.063659 & 29.32 \\
\hline & \multirow{2}{*}{$\mathrm{SBPSO}_{\mathrm{m} 5, \tau=10}$} & $\bar{x}$ & 43 & 4 & 0.008497 & 0.000985 & 0.000413 & 0.000326 & 0.858660 & 159.18 \\
\hline & & $\sigma$ & 0 & 0 & 0.000013 & 0.000010 & 0.000108 & 0.000018 & 0.065767 & 62.19 \\
\hline \multirow[t]{8}{*}{ FTSE 100} & \multirow{2}{*}{$\mathrm{SBPSO}_{\mathrm{m} 4}$} & $\bar{x}$ & 30 & 4 & 0.006684 & 0.000786 & 0.000282 & 0.000237 & 0.745490 & $\overline{16.97}$ \\
\hline & & $\sigma$ & 0 & 0 & 0.000007 & 0.000001 & 0.000050 & 0.000006 & 0.045856 & 3.14 \\
\hline & \multirow{2}{*}{$\mathrm{SBPSO}_{\mathrm{m} 5, \tau=3}$} & $\bar{x}$ & 30 & 4 & 0.006685 & 0.000786 & 0.000275 & 0.000239 & 0.746774 & 50.08 \\
\hline & & $\sigma$ & 0 & 0 & 0.000007 & 0.000001 & 0.000040 & 0.000007 & 0.049173 & 19.30 \\
\hline & \multirow{2}{*}{$\mathrm{SBPSO}_{\mathrm{m} 5, \tau=5}$} & $\bar{x}$ & 30 & 4 & 0.006683 & 0.000787 & 0.000284 & 0.000237 & 0.741699 & 69.80 \\
\hline & & $\sigma$ & 0 & 0 & 0.000008 & 0.000001 & 0.000048 & 0.000007 & 0.048705 & 26.82 \\
\hline & \multirow{2}{*}{$\mathrm{SBPSO}_{\mathrm{m} 5, \tau=10}$} & $\bar{x}$ & 30 & 4 & 0.006684 & 0.000785 & 0.000269 & 0.000236 & 0.758768 & 97.80 \\
\hline & & $\sigma$ & 0 & 0 & 0.000012 & 0.000005 & 0.000040 & 0.000006 & 0.035666 & 53.43 \\
\hline \multirow[t]{8}{*}{ S\&P 100} & \multirow[t]{2}{*}{$\mathrm{SBPSO}_{\mathrm{m} 4}$} & $\bar{x}$ & 44 & 6 & 0.007736 & 0.001275 & 0.000361 & 0.000253 & 0.865971 & 19.44 \\
\hline & & $\sigma$ & 0 & 0 & 0.000013 & 0.000023 & 0.000069 & 0.000008 & 0.041210 & 3.15 \\
\hline & \multirow{2}{*}{$\mathrm{SBPSO}_{\mathrm{m} 5, \tau=3}$} & $\bar{x}$ & 44 & 6 & 0.007736 & 0.001276 & 0.000363 & 0.000251 & 0.889834 & 64.89 \\
\hline & & $\sigma$ & 1 & 0 & 0.000012 & 0.000021 & 0.000071 & 0.000007 & 0.044258 & 21.45 \\
\hline & \multirow{2}{*}{$\mathrm{SBPSO}_{\mathrm{m} 5, \tau=5}$} & $\bar{x}$ & 44 & 6 & 0.007731 & 0.001268 & 0.000367 & 0.000252 & 0.869455 & 82.25 \\
\hline & & $\sigma$ & 0 & 0 & 0.000011 & 0.000015 & 0.000063 & 0.000005 & 0.039291 & 32.86 \\
\hline & \multirow{2}{*}{$\mathrm{SBPSO}_{\mathrm{m} 5, \tau=10}$} & $\bar{x}$ & 44 & 6 & 0.007733 & 0.001273 & 0.000366 & 0.000251 & 0.869151 & 135.20 \\
\hline & & $\sigma$ & 0 & 0 & 0.000012 & 0.000017 & 0.000060 & 0.000005 & 0.038260 & 69.21 \\
\hline \multirow[t]{8}{*}{ Nikkei 225} & \multirow[t]{2}{*}{$\mathrm{SBPSO}_{\mathrm{m} 4}$} & $\bar{x}$ & 44 & 4 & 0.003305 & 0.000790 & 0.000323 & 0.000230 & 0.908527 & 49.23 \\
\hline & & $\sigma$ & 1 & 0 & 0.000020 & 0.000017 & 0.000080 & 0.000008 & 0.036250 & 5.68 \\
\hline & \multirow[t]{2}{*}{$\mathrm{SBPSO}_{\mathrm{m} 5, \tau=3}$} & $\bar{x}$ & 43 & 4 & 0.003305 & 0.000788 & 0.000314 & 0.000229 & 0.908582 & 272.27 \\
\hline & & $\sigma$ & 1 & 0 & 0.000023 & 0.000018 & 0.000066 & 0.000010 & 0.047458 & 18.88 \\
\hline & $\mathrm{SBPSO}_{\mathrm{m} 5, \tau=5}$ & $\bar{x}$ & 44 & 4 & 0.003306 & 0.000791 & 0.000306 & 0.000231 & 0.921503 & 393.48 \\
\hline & & $\sigma$ & 1 & 0 & 0.000021 & 0.000014 & 0.000064 & 0.000008 & 0.037051 & 25.03 \\
\hline & $\mathrm{SBPSO}_{\mathrm{m} 5, \tau=10}$ & $\bar{x}$ & 44 & 4 & 0.003308 & 0.000792 & 0.000297 & 0.000226 & 0.927029 & 767.74 \\
\hline & & $\sigma$ & 1 & 0 & 0.000014 & 0.000013 & 0.000065 & 0.000009 & 0.042172 & 54.28 \\
\hline
\end{tabular}

Surprisingly, $\mathrm{SBPSO}_{\mathrm{m} 5}$ did not reduce the diversity of the set-particles despite the selection bias of the tournament as shown in Figures 6.6, 6.7 and 6.8. In fact, the diversity trends of $\mathrm{SBPSO}_{\mathrm{m} 5}$ for all tournament sizes were 
CHAPTER 6. SET-BASED PARTICLE SWARM OPTIMIZATION IMPROVEMENT AND DIVERSITY ANALYSIS

identical to that of $\mathrm{SBPSO}_{\mathrm{m} 4}$. Therefore, it can be said that the reintroduction of tournament selection was not a worthwhile modification and will not be used going forward.

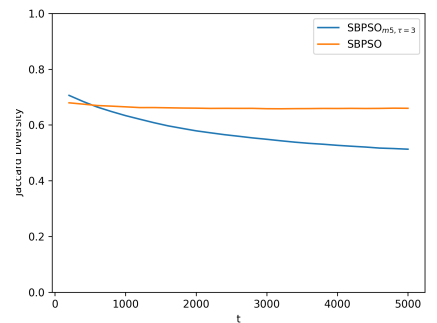

(a) Hang Seng

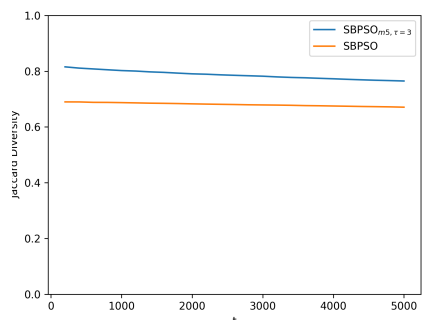

(b) DAX 100

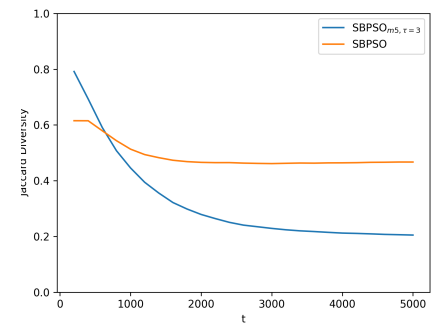

(c) FTSE 100

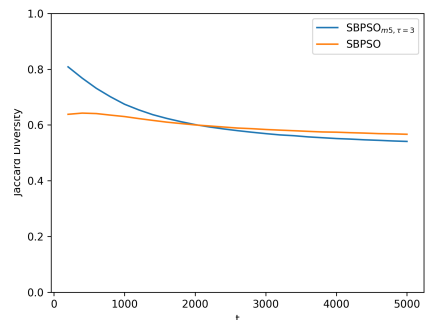

(d) S\&P 100

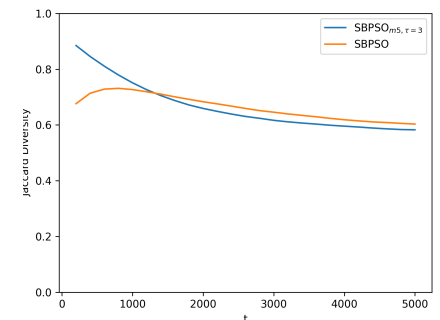

(e) Nikkei 225

Figure 6.6: Average Jaccard Diversity over Time for $\mathrm{SBPSO}_{\mathrm{m} 5, \tau=3}$

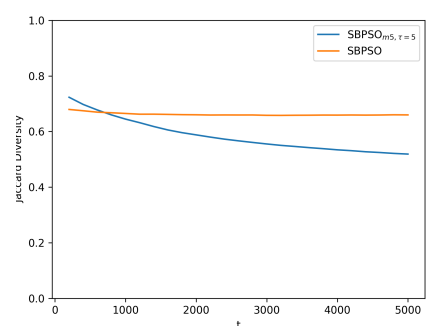

(a) Hang Seng

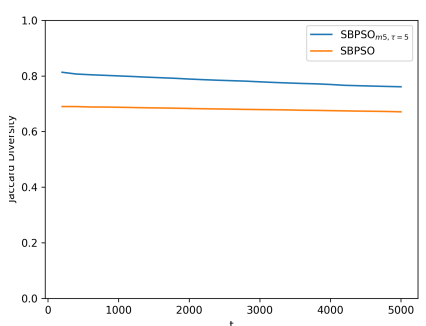

(b) DAX 100

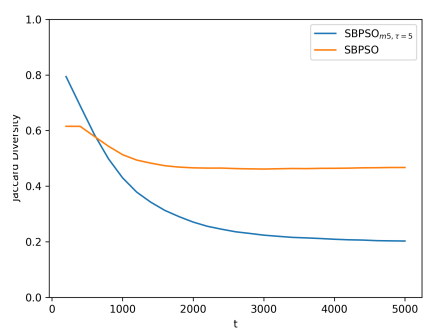

(c) FTSE 100

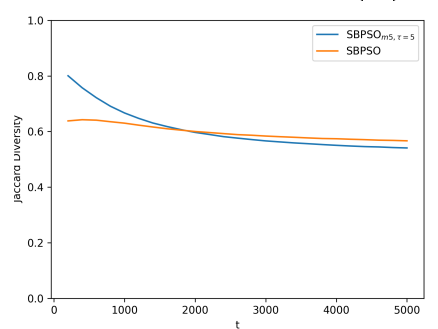

(d) S\&P 100

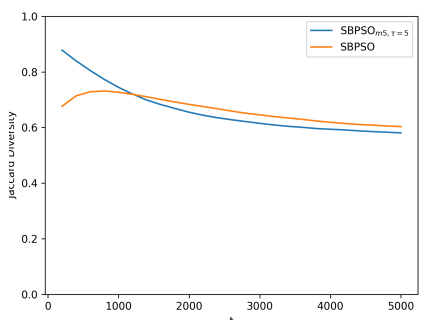

(e) Nikkei 225

Figure 6.7: Average Jaccard Diversity over Time for $\mathrm{SBPSO}_{\mathrm{m} 5, \tau=5}$ 


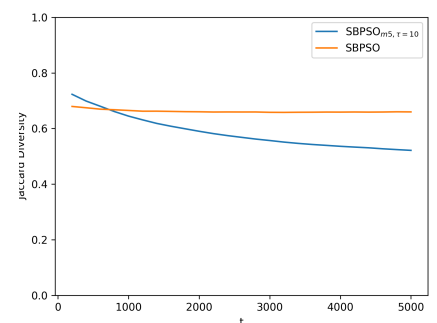

(a) Hang Seng

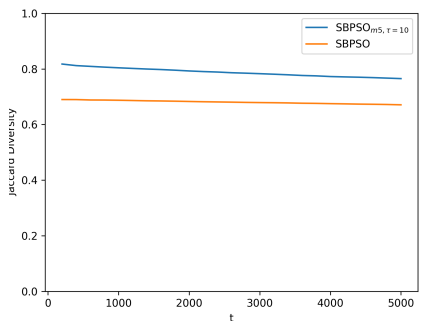

(b) DAX 100

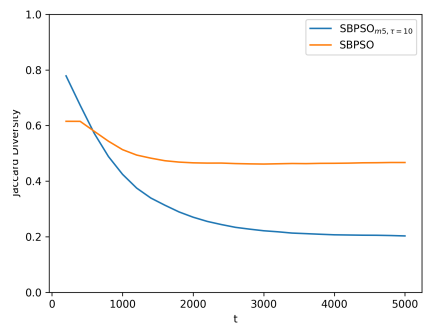

(c) FTSE 100

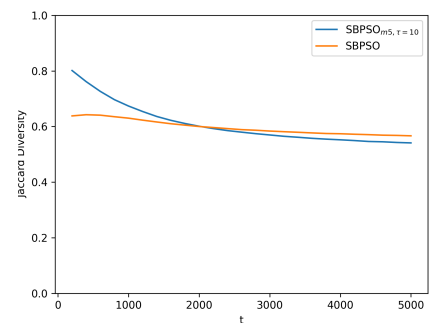

(d) S\&P 100

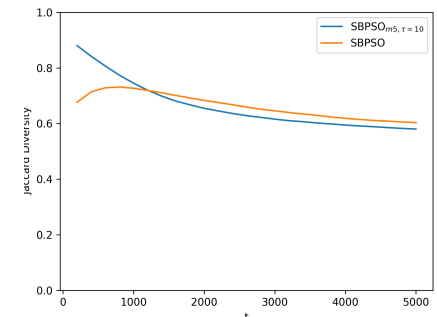

(e) Nikkei 225

Figure 6.8: Average Jaccard Diversity over Time for $\mathrm{SBPSO}_{\mathrm{m} 5, \tau=10}$

\subsubsection{Exploration Balance Coefficient}

The previous modification attempted to control the amount of diversity added to the set-particle's positions by means of tournament selection. However, there were no gains in performance and it severely impacted the time to reach the stopping condition. The exploration balance coefficient modification continues the endeavour to improve the balance between exploration and exploitation behaviour of SBPSO while simultaneously improving performance.

The cognitive, $\left(Y_{i}(t) \ominus X_{i}(t)\right)$, and social, $\left(\hat{Y}_{i}(t) \ominus X_{i}(t)\right)$, components of the SBPSO velocity update equation (6.1) guide set-particles towards previously found good areas of the search space. If not properly controlled, the cognitive and social components could lead towards premature convergence of the swarm since the set-particles would become more and more similar sooner. Conversely, the third component (the exploration component) of the SBPSO velocity update equation (6.1) injects diversity into the swarm by adding elements outside of $X(t) \cup Y(t) \cup \hat{Y}(t)$ to $X(t+1)$. Too much diversity would result in a constant state of exploration, and the swarm would be unable to converge to a single solution. Ideally, the time between these two stages should be balanced, allowing for time to explore the search space in the beginning of the search, and gradually exploiting fruitful areas over time. With this ideal scenario in mind, this modification adds a linearly increasing control parameter, $\lambda_{c}$, to moderate the exploitation of the cognitive and social components, and exploration caused by the exploration component. The linearly increasing control parameter, referred to as the exploration balance coefficient, initially 
zero, is defined as

$$
\lambda_{c}(t)=\frac{t}{n_{t}}
$$

where $n_{t}$ is the maximum number of iterations. At the start of the search, i.e. when $t=0, \lambda_{c}$ is set to zero. The updated velocity equation for this modification is

$$
\begin{aligned}
V_{i}(t+1)= & \lambda_{c}(t) r_{1} \otimes\left(Y_{i}(t) \ominus X_{i}(t)\right) \\
& \oplus \lambda_{c}(t) r_{2} \otimes\left(\hat{Y}_{i}(t) \ominus X_{i}(t)\right) \\
& \oplus\left(1-\lambda_{c}(t)\right) r_{3} \otimes A_{i}(t)
\end{aligned}
$$

where $r_{1}, r_{2}$, and $r_{3}$ are random values, each sampled from a uniform distribution in the range $[0,2]$. The range $[0,2]$ is used instead of $[0,1]$ to ensure that each component is not too small as a result of $\lambda_{c}(t)$.

When $\lambda_{c}$ is equal to zero, the social and cognitive guides have no influence on the set-particle's next position whereas the influence of the exploration component is at a maximum. Thereafter, the influence of the social and cognitive guides increases linearly over time while the exploration guide decreases proportionately. This means that the set-swarm will be encouraged to explore the asset search space at the beginning of the search. Then as time goes on the set-swarm will be encouraged to refine solutions as the influence of the social and cognitive guides increases while the exploration guide decreases. When $\lambda_{c}$ is equal to one, the influence of the social and cognitive guides is at a maximum and the exploration guide has no influence. It is expected that this modification will not only improve the exploration and exploitation behaviour of SBPSO, but also the quality of solutions obtained.

Figure 6.9 shows smooth downwards trends from large Jaccard diversity values to Jaccard diversity values close to zero were obtained. In contrast, the Jaccard diversity values of SBPSO were consistently high and never reached zero. Thus, the introduction of $\lambda_{c}$ allows the set-swarm to explore the search space early on and to refine solutions as time goes on, until eventually the swarm converges. 
CHAPTER 6. SET-BASED PARTICLE SWARM OPTIMIZATION IMPROVEMENT AND DIVERSITY ANALYSIS

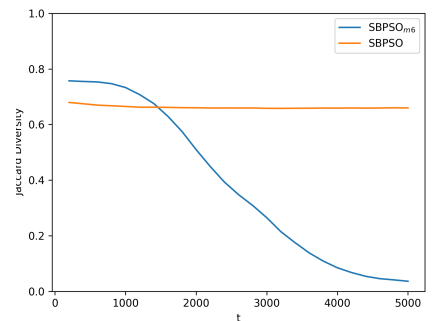

(a) Hang Seng

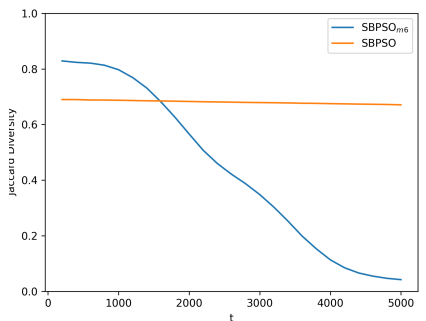

(b) DAX 100

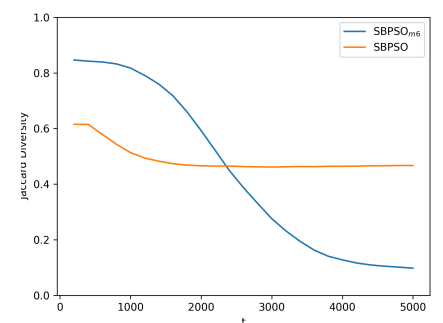

(c) FTSE 100

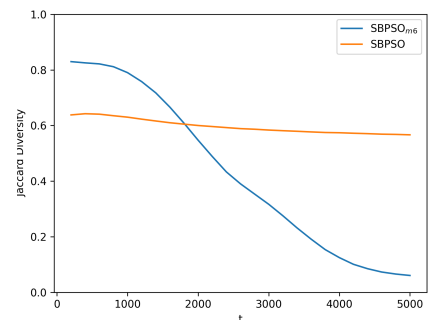

(d) S\&P 100

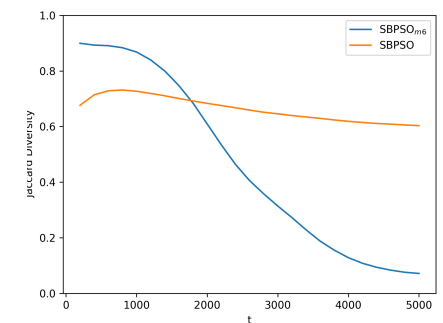

(e) Nikkei 225

Figure 6.9: Average Jaccard Diversity over Time for $\mathrm{SBPSO}_{\mathrm{m} 6}$

The average and standard deviations of the results obtained by $\mathrm{SBPSO}_{\mathrm{m} 6}$ were typically similar to, if not better than those obtained $\mathrm{SBPSO}_{\mathrm{m}}$, as seen in Table 6.7. For example, the number of optimal solutions obtained by either algorithm was the same, and the average size of those optimal solutions was the same except for FTSE 100. Statistical significance tests show that profitability of $\mathrm{SBPSO}_{\mathrm{m} 6}$ was the same or worse than that of $\mathrm{SBPSO}_{\mathrm{m} 4}$, although the average values were very close. However, statistical significance tests also show that $\mathrm{SBPSO}_{\mathrm{m} 6}$ was as or less risky than $\mathrm{SBPSO}_{\mathrm{m} 4}$. Furthermore, $\mathrm{SBPSO}_{\mathrm{m}}$ was statistically significantly the same or better than $\mathrm{SBPSO}_{\mathrm{m} 4}$ for the Pareto optimality measures, namely GD, IGD and HV. Lastly, $\mathrm{SBPSO}_{\mathrm{m} 6}$ was faster to reach the stopping condition than $\mathrm{SBPSO}_{\mathrm{m}}$, except for Nikkei 225 where $\mathrm{SBPSO}_{\mathrm{m} 6}$ was slower by approximately 14 seconds. 
CHAPTER 6. SET-BASED PARTICLE SWARM OPTIMIZATION IMPROVEMENT AND DIVERSITY ANALYSIS

Table 6.7: $\mathrm{SBPSO}_{\mathrm{m} 4}$ versus $\mathrm{SBPSO}_{\mathrm{m} 6}$ : Mean and standard deviations for each performance measure

\begin{tabular}{|c|c|c|c|c|c|c|c|c|c|c|}
\hline & Algorithm & Result & $\mathbf{N}$ & $K$ & $R$ & $\bar{\sigma}$ & GD & IGD & HV & Time \\
\hline \multirow[t]{4}{*}{ Hang Seng } & \multirow[t]{2}{*}{$\mathrm{SBPSO}_{\mathrm{m} 4}$} & $\bar{x}$ & 33 & 3 & 0.007614 & 0.001900 & 0.000274 & 0.000217 & 0.785971 & 7.04 \\
\hline & & $\sigma$ & 1 & 0 & 0.000007 & 0.000005 & 0.000088 & 0.000006 & 0.019312 & 1.89 \\
\hline & \multirow[t]{2}{*}{$\mathrm{SBPSO}_{\mathrm{m} 6}$} & $\bar{x}$ & 33 & 3 & 0.007607 & 0.001897 & 0.000218 & 0.000212 & 0.781949 & 4.52 \\
\hline & & $\sigma$ & 0 & 0 & 0.000003 & 0.000000 & 0.000045 & 0.000002 & 0.026784 & 2.87 \\
\hline \multirow[t]{4}{*}{ DAX 100} & \multirow[t]{2}{*}{$\mathrm{SBPSO}_{\mathrm{m} 4}$} & $\bar{x}$ & 43 & 4 & 0.008498 & 0.000986 & 0.000413 & 0.000330 & 0.851289 & 12.60 \\
\hline & & $\sigma$ & 1 & 0 & 0.000020 & 0.000013 & 0.000119 & 0.000017 & 0.068197 & 2.69 \\
\hline & \multirow[t]{2}{*}{$\mathrm{SBPSO}_{\mathrm{m} 6}$} & $\bar{x}$ & 43 & 4 & 0.008469 & 0.000972 & 0.000250 & 0.000306 & 0.878138 & 8.99 \\
\hline & & $\sigma$ & 0 & 0 & 0.000006 & 0.000000 & 0.000061 & 0.000008 & 0.065429 & 3.19 \\
\hline \multirow[t]{4}{*}{ FTSE 100} & \multirow[t]{2}{*}{$\mathrm{SBPSO}_{\mathrm{m} 4}$} & $\bar{x}$ & 30 & 4 & 0.006684 & 0.000786 & 0.000282 & 0.000237 & 0.745490 & 16.97 \\
\hline & & $\sigma$ & 0 & 0 & 0.000007 & 0.000001 & 0.000050 & 0.000006 & 0.045856 & 3.14 \\
\hline & \multirow[t]{2}{*}{$\mathrm{SBPSO}_{\mathrm{m} 6}$} & $\bar{x}$ & 30 & 5 & 0.006683 & 0.000785 & 0.000247 & 0.000235 & 0.741994 & 10.57 \\
\hline & & $\sigma$ & 0 & 0 & 0.000007 & 0.000001 & 0.000038 & 0.000007 & 0.049170 & 3.55 \\
\hline \multirow[t]{4}{*}{ S\&P 100} & \multirow[t]{2}{*}{$\mathrm{SBPSO}_{\mathrm{m} 4}$} & $\bar{x}$ & 44 & 6 & 0.007736 & 0.001275 & 0.000361 & 0.000253 & 0.865971 & 19.44 \\
\hline & & $\sigma$ & 0 & 0 & 0.000013 & 0.000023 & 0.000069 & 0.000008 & 0.041210 & 3.15 \\
\hline & \multirow[t]{2}{*}{$\mathrm{SBPSO}_{\mathrm{m} 6}$} & $\bar{x}$ & 44 & 6 & 0.007716 & 0.001248 & 0.000275 & 0.000247 & 0.875057 & 16.45 \\
\hline & & $\sigma$ & 0 & 0 & 0.000006 & 0.000001 & 0.000044 & 0.000003 & 0.044175 & 2.78 \\
\hline \multirow[t]{4}{*}{ Nikkei 225} & \multirow[t]{2}{*}{$\mathrm{SBPSO}_{\mathrm{m} 4}$} & $\bar{x}$ & 44 & 4 & 0.003305 & 0.000790 & 0.000323 & 0.000230 & 0.908527 & 49.23 \\
\hline & & $\sigma$ & 1 & 0 & 0.000020 & 0.000017 & 0.000080 & 0.000008 & 0.036250 & 5.68 \\
\hline & \multirow[t]{2}{*}{$\mathrm{SBPSO}_{\mathrm{m} 6}$} & $\bar{x}$ & 44 & 4 & 0.003310 & 0.000794 & 0.000260 & 0.000223 & 0.916014 & 63.19 \\
\hline & & $\sigma$ & 0 & 0 & 0.000007 & 0.000001 & 0.000060 & 0.000005 & 0.047097 & 6.13 \\
\hline
\end{tabular}

The results from Table 6.7 and Figure 6.9 clearly show that this proposed modification to introduce a linearly increasing control parameter to the velocity update equation, was a significant improvement. As a consequence of this modification, parameter tuning is no longer necessary, which can often be a costly investigation in terms of computational resources and time. For these reasons, this proposed modification was certainly worth keeping.

\subsection{Adaptive Coordinate Descent Weight Optimization}

This section presents ACD as an alternative weight optimizer for the improved $\mathrm{SBPSO}_{\mathrm{m} 6}$ approach 113. Section 6.4.1 gives the necessary background on ACD. The motivation for using ACD as a weight optimizer is given in Section 6.4.2. Section 6.4.3 discusses the results.

\subsubsection{Adaptive Coordinate Descent}

Loshchilov et al. developed ACD in 2011 and found it to be a good approach to several optimization problems [113]. ACD borrows and generalises the adaptive technique used in the covariance matrix adaptation evolution strategy (CMA-ES) [74, such that it can be applied to any optimization algorithm. Adaptive encoding (AE), given in Algorithm 10, iteratively transforms the 
search system of the objective function so that it is as similar as possible to the Sphere function, which is known to be simple for analysis and optimization [113. This process results in a very efficient search process for non-separable problems e.g. portfolio optimization.

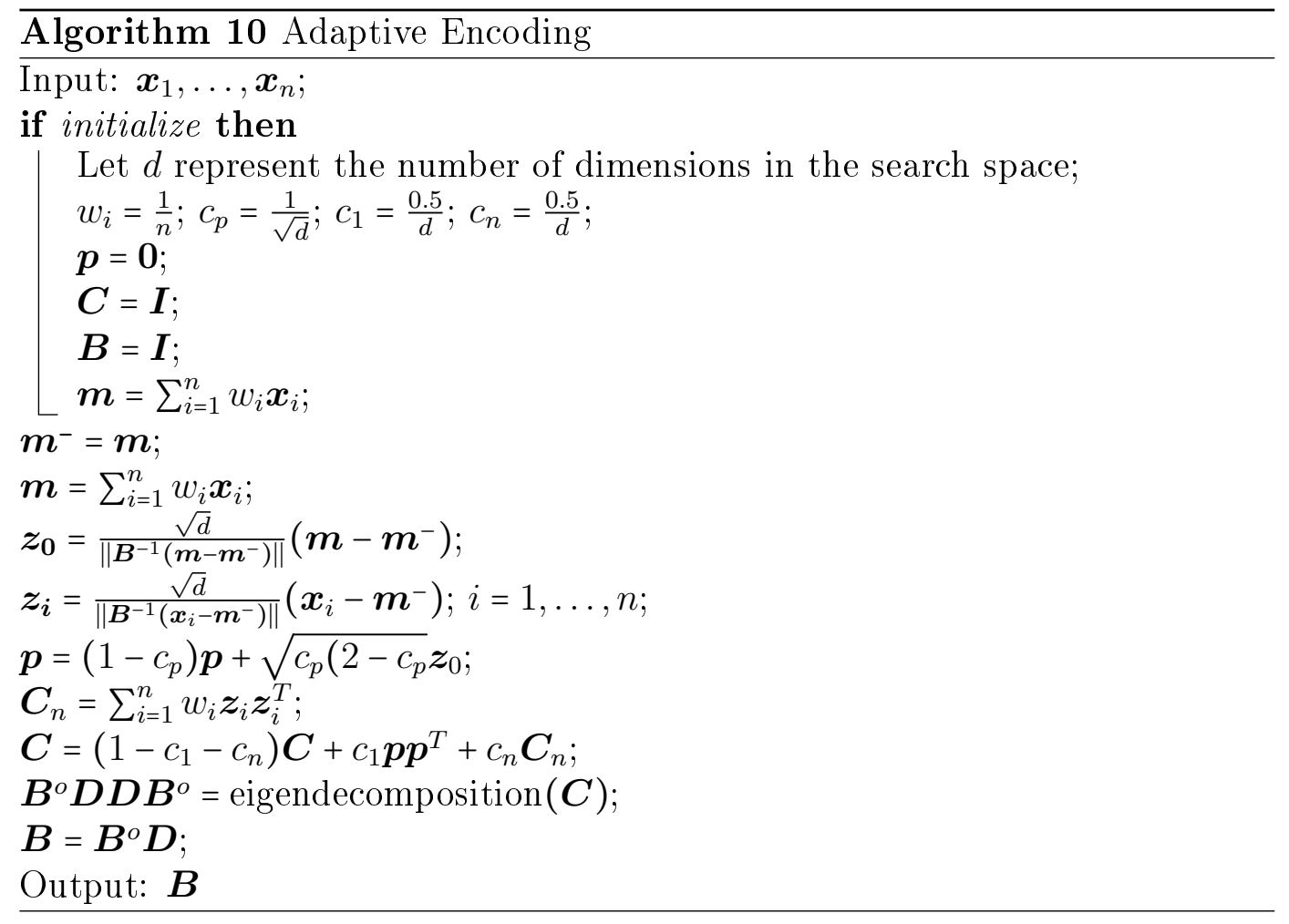

AE is similar to principal component analysis (PCA) where the goal is to convert a set of possibly correlated variables into a set of uncorrelated variables, called principal components. The principal components are the eigenvectors of the covariance matrix, $\boldsymbol{C}$. The principal components are determined at each iteration by the eigendecomposition of the current covariance matrix $\boldsymbol{C}$.

Coordinate descent (CD) is based on the idea that an $n$-dimensional separable optimization problem can be broken up into $n$ one-dimensional optimization problems. CD iteratively optimizes each independent variable while keeping the other decision variables fixed.

ACD, summarised in Algorithm 11, is CD that makes use of AE. Thus, $\mathrm{ACD}$ is an improvement over $\mathrm{CD}$ as it can handle non-separable problems. ACD has been shown to be competitive with CMA-ES algorithms, and in some cases even up to two times faster [113]. Furthermore, ACD has also been shown to be more advantageous than traditional CD. Algorithm 11 describes the ACD algorithm in which adaptive encoding is applied to coordinate descent.

The lower and upper bounds for the search are given by $\boldsymbol{a}$ and $\boldsymbol{b}$, respectively. The parameters $k_{\text {shrink }}$ and $k_{\text {grow }}$ determine the size of the interval along 
which the ACD algorithm should search for the next best solution. When a solution better than the current best is found on either end of the search interval, the interval size is expanded by a factor of $k_{\text {grow }}$ in that coordinate. Otherwise, the search interval is symmetrically decreased in size by a factor of $k_{\text {shrink }}$. These parameters therefore control the exploration-exploitation tradeoff the search algorithm and should be tuned per problem.

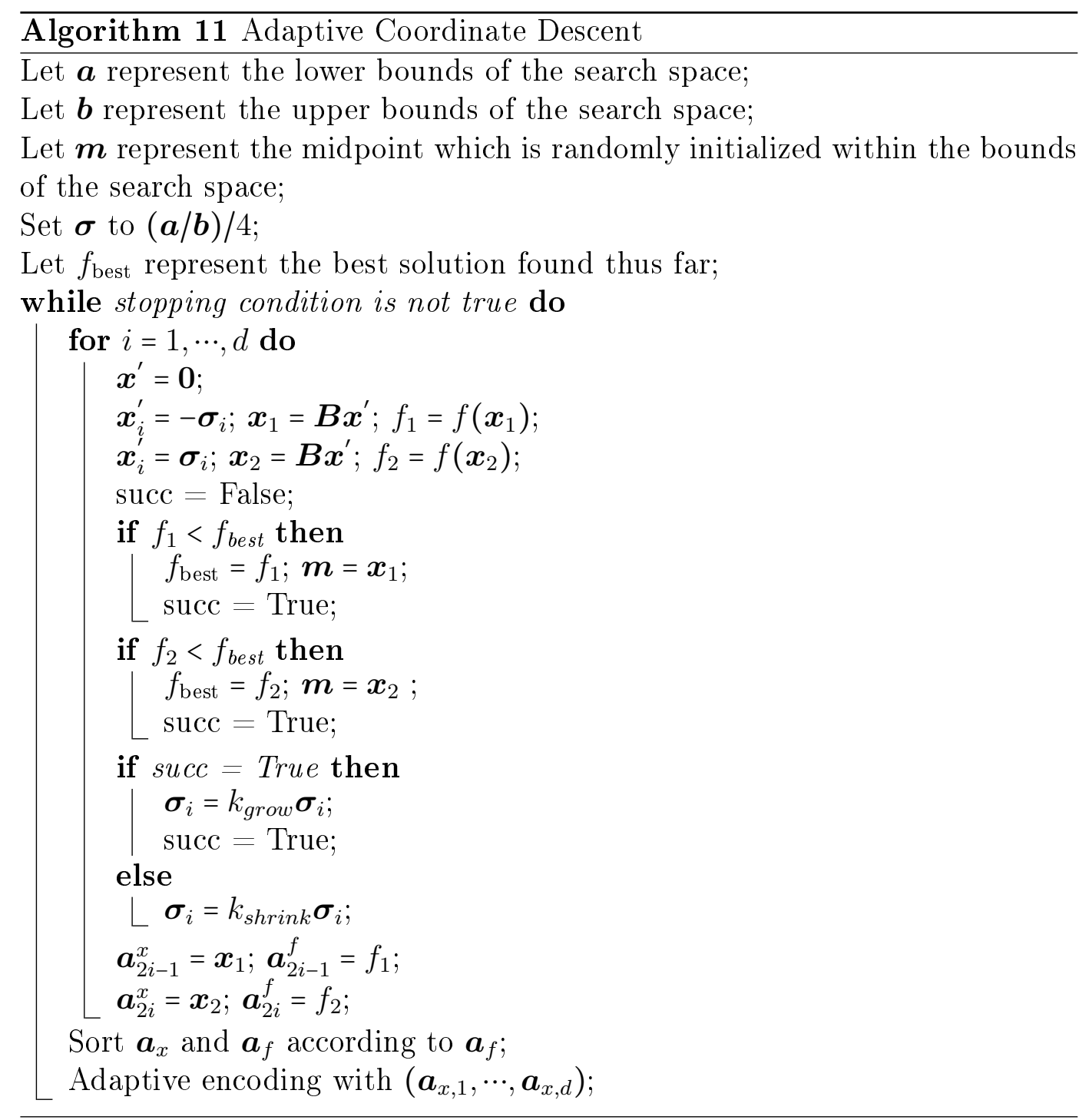

\subsubsection{Motivation}

Portfolio optimization is a non-separable problem since risk (equation (3.2)) is calculated using variance which is not separable. Additionally, portfolio optimization is made further non-separable because the asset weights are dependent on each other. Considering equation (3.4), as one weight increases, the other weights decrease. The inverse is also true. ACD has been shown to 
be an efficient optimizer for single-objective optimization problems [113]. ACD is capable of optimizing non-separable problems as it iteratively transforms the search space to maximize the decorrelation of decision variables with respect to the objective function. ACD is also a completely deterministic approach, unlike PSO. Deterministic methods ensure, after a finite time, an approximation of a global optimum will be found [101. The decision to use ACD as weight optimizer is motivated by the aforementioned characteristics. It is expected that ACD will improve the quality of the solutions obtained by SBPSO. For convenience purposes, the ACD variant of SBPSO is referred to as $\mathrm{SBPSO}_{\mathrm{ACD}}$.

\subsubsection{Results}

Like $\mathrm{SBPSO}_{\mathrm{m} 6}, \mathrm{SBPSO}_{\mathrm{ACD}}$ balances time between exploration and exploitation well as seen in Figure 6.10. In fact, the Jaccard diversity values at the start of the search for both algorithms are very similar, but $\mathrm{SBPSO}_{\mathrm{ACD}}$ ends the search with Jaccard diversity values much closer to zero than $\mathrm{SBPSO}_{\mathrm{m} 6}$. Thus, the convergence behaviour of $\mathrm{SBPSO}_{\mathrm{ACD}}$ was better than that of $\mathrm{SBPSO}_{\mathrm{m} 6}$. Table 6.8 shows that the average risk, GD and IGD results obtained by $\mathrm{SBPSO}_{\mathrm{ACD}}$ were better than those of $\mathrm{SBPSO}_{\mathrm{m} 6}$, except for DAX 100 where $\mathrm{SBPSO}_{\mathrm{m} 6}$ had a lower IGD score on average. $\mathrm{SBPSO}_{\mathrm{ACD}}$ performed particularly well for the Hang Seng benchmark problem where it was statistically significantly the same or better than $\mathrm{SBPSO}_{\mathrm{m} 6}$. Statistical significance tests also show that $\mathrm{SBPSO}_{\mathrm{ACD}}$ was better than $\mathrm{SBPSO}_{\mathrm{m} 6}$ at obtaining solutions closer to the true POF than $\mathrm{SBPSO}_{\mathrm{m} 6}$. However, $\mathrm{SBPSO}_{\mathrm{m} 6}$ was statistically significantly faster than $\mathrm{SBPSO}_{\mathrm{ACD}}$ for all benchmark problems, except for Hang Seng. The time that $\mathrm{SBPSO}_{\mathrm{ACD}}$ took to reach the stopping condition increased as the dimensionality of the problem increased. One of the motivations for SBPSO for portfolio optimization was to address the scalability problems of other algorithms for portfolio optimization. Although $\mathrm{SBPSO}_{\mathrm{ACD}}$ was scalable in that it found good quality results despite the dimensionality of the problem increasing, it did not find solutions to larger problems in a timely manner. For example, $\mathrm{SBPSO}_{\mathrm{ACD}}$ took approximately 60 seconds to find solutions to the FTSE problem, whereas $\mathrm{SBPSO}_{\mathrm{m} 6}$ took 10 seconds. For Nikkei, the largest problem, $\mathrm{SBPSO}_{\mathrm{ACD}}$ took about 340 seconds while $\mathrm{SBPSO}_{\mathrm{m} 6}$ took 60 seconds. Overall the results show that $\mathrm{SBPSO}_{\mathrm{ACD}}$ is a competitive approach to $\mathrm{SBPSO}_{\mathrm{m} 6}$. 
CHAPTER 6. SET-BASED PARTICLE SWARM OPTIMIZATION IMPROVEMENT AND DIVERSITY ANALYSIS

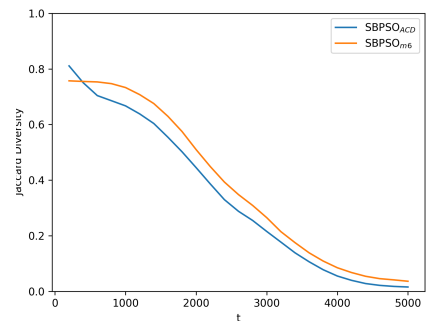

(a) Hang Seng

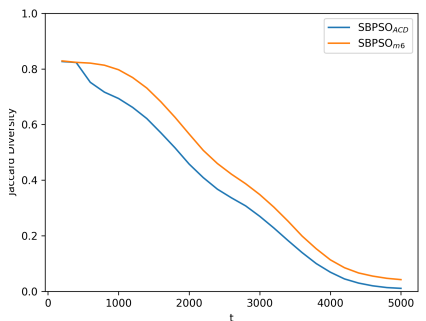

(b) DAX 100

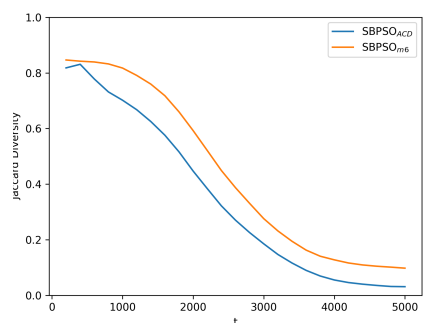

(c) FTSE 100

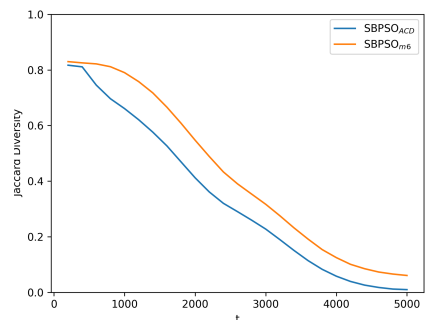

(d) S\&P 100

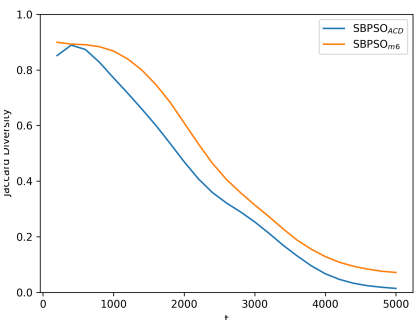

(e) Nikkei 225

Figure 6.10: Average Jaccard Diversity over Time for $\mathrm{SBPSO}_{\mathrm{ACD}}$

Table 6.8: $\mathrm{SBPSO}_{\mathrm{m} 6}$ versus $\mathrm{SBPSO}_{\mathrm{ACD}}$ : Mean and standard deviations for each performance measure

\begin{tabular}{|c|c|c|c|c|c|c|c|c|c|c|}
\hline & Algorithm & Result & $\mathbf{N}$ & $K$ & $R$ & $\bar{\sigma}$ & GD & IGD & HV & Time \\
\hline \multirow[t]{4}{*}{ Hang Seng } & $\mathrm{SBPSO}_{\mathrm{m} 6}$ & $\bar{x}$ & 33 & 3 & 0.007607 & 0.001897 & 0.000218 & 0.000212 & 0.781949 & 4.52 \\
\hline & & $\sigma$ & 0 & 0 & 0.000003 & 0.000000 & 0.000045 & 0.000002 & 0.026784 & 2.87 \\
\hline & $\mathrm{SBPSO}_{\mathrm{ACD}}$ & $\bar{x}$ & 34 & 3 & 0.007600 & 0.001891 & 0.000190 & 0.000210 & 0.814630 & 2.17 \\
\hline & & $\sigma$ & 0 & 0 & 0.000025 & 0.000021 & 0.000003 & 0.000000 & 0.000000 & 2.31 \\
\hline \multirow[t]{4}{*}{ DAX 100} & $\mathrm{SBPSO}_{\mathrm{m} 6}$ & $\bar{x}$ & 43 & 4 & 0.008474 & 0.000973 & 0.000299 & 0.000311 & 0.863570 & 8.99 \\
\hline & & $\sigma$ & 0 & 0 & 0.000010 & 0.000002 & 0.000090 & 0.000010 & 0.067062 & 3.19 \\
\hline & $\mathrm{SBPSO}_{\mathrm{ACD}}$ & $\bar{x}$ & 42 & 4 & 0.008460 & 0.000971 & 0.000209 & 0.000346 & 0.804378 & 28.09 \\
\hline & & $\sigma$ & 9 & 0 & 0.000001 & 0.000000 & 0.000000 & 0.000000 & 0.165428 & 45.21 \\
\hline \multirow[t]{4}{*}{ FTSE 100} & $\mathrm{SBPSO}_{\mathrm{m} 6}$ & $\bar{x}$ & 30 & 5 & 0.006683 & 0.000785 & 0.000247 & 0.000235 & 0.741994 & 10.57 \\
\hline & & $\sigma$ & 0 & 0 & 0.000007 & 0.000001 & 0.000038 & 0.000007 & 0.049170 & 3.55 \\
\hline & $\mathrm{SBPSO}_{\mathrm{ACD}}$ & $\bar{x}$ & 29 & 5 & 0.006655 & 0.000775 & 0.000169 & 0.000232 & 0.711504 & 35.75 \\
\hline & & $\sigma$ & 1 & 0 & 0.000024 & 0.000011 & 0.000019 & 0.000001 & 0.051801 & 61.04 \\
\hline \multirow[t]{4}{*}{ S\&P 100} & $\mathrm{SBPSO}_{\mathrm{m} 6}$ & $\bar{x}$ & 44 & 6 & 0.007716 & 0.001248 & 0.000275 & 0.000247 & 0.875057 & 16.45 \\
\hline & & $\sigma$ & 0 & 0 & 0.000006 & 0.000001 & 0.000044 & 0.000003 & 0.044175 & 2.78 \\
\hline & $\mathrm{SBPSO}_{\mathrm{ACD}}$ & $\bar{x}$ & 44 & 6 & 0.007708 & 0.001244 & 0.000205 & 0.000242 & 0.872137 & 91.53 \\
\hline & & $\sigma$ & 0 & 0 & 0.000001 & 0.000000 & 0.000022 & 0.000001 & 0.049381 & 166.15 \\
\hline \multirow[t]{4}{*}{ Nikkei 225} & $\mathrm{SBPSO}_{\mathrm{m} 6}$ & $\bar{x}$ & 44 & 4 & 0.003310 & 0.000794 & 0.000260 & 0.000223 & 0.916014 & 63.19 \\
\hline & & $\sigma$ & 0 & 0 & 0.000007 & 0.000001 & 0.000060 & 0.000005 & 0.047097 & 6.13 \\
\hline & $\mathrm{SBPSO}_{\mathrm{ACD}}$ & $\bar{x}$ & 44 & 4 & 0.003305 & 0.000791 & 0.000139 & 0.000209 & 0.898166 & 342.28 \\
\hline & & $\sigma$ & 0 & 0 & 0.000001 & 0.000001 & 0.000027 & 0.000001 & 0.024326 & 59.95 \\
\hline
\end{tabular}

If one is willing to sacrifice time in order to obtain higher quality solutions, then $\mathrm{SBPSO}_{\mathrm{ACD}}$ is a compelling option. The performance of $\mathrm{SBPSO}_{\mathrm{ACD}}$ for portfolio optimization is compared with other algorithms in the next section. 


\subsection{Modified Set-based Particle Swarm Optimization for Portfolio Optimization in Comparison with Other Algorithms}

This section compares the performance of $\mathrm{SBPSO}_{\mathrm{m} 6}$ and $\mathrm{SBPSO}_{\mathrm{ACD}}$ against that of ABC, FA, GA, PSO and original SBPSO for portfolio optimization. Both $\mathrm{SBPSO}_{\mathrm{m} 6}$ and $\mathrm{SBPSO}_{\mathrm{ACD}}$ are used in this comparison as they represent the accumulative gain in performance over the original approach. $\mathrm{SBPSO}_{\mathrm{m}}$ has been shown to deliver good quality results quickly, while $\mathrm{SBPSO}_{\mathrm{ACD}}$ obtained better results than $\mathrm{SBPSO}_{\mathrm{m} 6}$, but was slower.

The Hang Seng results are given in Section 6.5.1. Section 6.5.2 discusses the DAX 100 results. Sections 6.5.3 and 6.5.4 present the FTSE and S\&P results respectively. The Nikkei 225 results are discussed in Section 6.5.5.

\subsubsection{Hang Seng}

Table 6.9 shows that the average results for risk, GD, IGD and $\mathrm{HV}$ of $\mathrm{SBPSO}_{\mathrm{m}}$ and $\mathrm{SBPSO}_{\mathrm{ACD}}$ were better than those of SBPSO. Furthermore, both of the modified SBPSOs were faster than the original SBPSO, which was already faster than the other algorithms. The rankings in Table 6.10 show that, overall, $\mathrm{SBPSO}_{\mathrm{ACD}}$ outperformed all other algorithms, a significant improvement over the original SBPSO. $\mathrm{SBPSO}_{\mathrm{m} 6}$ ranked third overall, also an improvement over the original approach. With regard to risk and return, $\mathrm{SBPSO}_{\mathrm{m} 6}$ and $\mathrm{SBPSO}_{\mathrm{ACD}}$ ranked similarly to the PSO. The original SBPSO on the other hand ranked second last for risk and return. Lastly, Figure 6.11 shows that $\mathrm{SBPSO}_{\mathrm{m} 6}$ and $\mathrm{SBPSO}_{\mathrm{ACD}}$ were also able to approximate the true POF as well as the other algorithms.

Table 6.9: Hang Seng results for each performance measure

\begin{tabular}{lccccccccc}
\hline Algorithm & Result & $\mathbf{N}$ & $K$ & $R$ & $\bar{\sigma}$ & GD & IGD & HV & Time \\
\hline ABC & $\bar{x}$ & 37 & 14 & 0.007848 & 0.002110 & 0.000176 & 0.000210 & 0.814630 & 11.04 \\
& $\sigma$ & 2 & 2 & 0.000142 & 0.000125 & 0.000009 & 0.000000 & 0.000000 & 0.41 \\
\hline FA & $\bar{x}$ & 38 & 5 & 0.006084 & 0.001783 & 0.003959 & 0.000556 & 0.748081 & 38.95 \\
& $\sigma$ & 4 & 1 & 0.000592 & 0.000268 & 0.000485 & 0.000085 & 0.028972 & 3.71 \\
\hline GA & $\bar{x}$ & 32 & 3 & 0.007576 & 0.001884 & 0.000279 & 0.000222 & 0.779399 & 18.10 \\
& $\sigma$ & 1 & 0 & 0.000052 & 0.000042 & 0.000065 & 0.000005 & 0.025320 & 0.64 \\
\hline PSO & $\bar{x}$ & 33 & 3 & 0.007607 & 0.001897 & 0.000219 & 0.000212 & 0.786254 & 15.02 \\
& $\sigma$ & 1 & 0 & 0.000003 & 0.000001 & 0.000049 & 0.000002 & 0.026135 & 0.69 \\
\hline SBPSO & $\bar{x}$ & 34 & 3 & 0.007614 & 0.001908 & 0.000463 & 0.000236 & 0.776926 & 9.37 \\
& $\sigma$ & 1 & 0 & 0.000022 & 0.000009 & 0.000141 & 0.000010 & 0.023747 & 0.53 \\
\hline SBPSO $_{\text {m6 }}$ & $\bar{x}$ & 33 & 3 & 0.007607 & 0.001897 & 0.000218 & 0.000212 & 0.781949 & 4.52 \\
& $\sigma$ & 0 & 0 & 0.000003 & 0.000000 & 0.000045 & 0.000002 & 0.026784 & 2.87 \\
\hline SBPSO $_{\text {ACD }}$ & $\bar{x}$ & 34 & 3 & 0.007600 & 0.001891 & 0.000190 & 0.000210 & 0.814630 & 2.17 \\
& $\sigma$ & 0 & 0 & 0.000025 & 0.000021 & 0.000003 & 0.000000 & 0.000000 & 2.31 \\
\hline
\end{tabular}


CHAPTER 6. SET-BASED PARTICLE SWARM OPTIMIZATION IMPROVEMENT AND DIVERSITY ANALYSIS

Table 6.10: Hang Seng rankings for each performance measure

\begin{tabular}{|c|c|c|c|c|c|c|c|c|c|c|}
\hline Algorithm & Result & $\mathbf{N}$ & $K$ & $R$ & $\bar{\sigma}$ & GD & IGD & HV & Time & Overall \\
\hline \multirow[t]{5}{*}{$\mathrm{ABC}$} & Wins & 5 & 0 & 6 & 0 & 6 & 5 & 5 & 3 & 30 \\
\hline & Losses & 0 & 6 & 0 & 6 & 0 & 0 & 0 & 3 & 15 \\
\hline & Draws & 1 & 0 & 0 & 0 & 0 & 1 & 1 & 0 & 3 \\
\hline & Difference & 5 & -6 & 6 & -6 & 6 & 5 & 5 & 0 & 15 \\
\hline & Rank & 1 & 6 & 1 & 6 & 1 & 1 & 1 & 4 & 2 \\
\hline \multirow[t]{5}{*}{ FA } & Wins & 5 & 1 & 0 & 5 & 0 & 0 & 0 & 0 & 11 \\
\hline & Losses & 0 & 5 & 6 & 0 & 6 & 6 & 6 & 6 & 35 \\
\hline & Draws & 1 & 0 & 0 & 1 & 0 & 0 & 0 & 0 & 2 \\
\hline & Difference & 5 & -4 & -6 & 5 & -6 & -6 & -6 & -6 & -24 \\
\hline & Rank & 1 & 5 & 6 & 1 & 6 & 5 & 5 & 7 & 7 \\
\hline \multirow[t]{5}{*}{ GA } & Wins & 0 & 2 & 1 & 2 & 2 & 2 & 1 & 1 & 11 \\
\hline & Losses & 6 & 4 & 4 & 0 & 4 & 4 & 3 & 5 & 30 \\
\hline & Draws & 0 & 0 & 1 & 4 & 0 & 0 & 2 & 0 & 7 \\
\hline & Difference & -6 & -2 & -3 & 2 & -2 & -2 & -2 & -4 & -19 \\
\hline & Rank & 4 & 4 & 5 & 3 & 4 & 3 & 4 & 6 & 6 \\
\hline \multirow[t]{5}{*}{ PSO } & Wins & 1 & 3 & 2 & 2 & 3 & 3 & 2 & 2 & 18 \\
\hline & Losses & 3 & 3 & 1 & 2 & 2 & 2 & 2 & 4 & 19 \\
\hline & Draws & 2 & 0 & 3 & 2 & 1 & 1 & 2 & 0 & 11 \\
\hline & Difference & -2 & 0 & 1 & 0 & 1 & 1 & 0 & -2 & -1 \\
\hline & Rank & 3 & 3 & 3 & 4 & 3 & 2 & 2 & 5 & 4 \\
\hline \multirow[t]{5}{*}{ SBPSO } & Wins & 1 & 6 & 3 & 1 & 1 & 1 & 1 & 4 & 18 \\
\hline & Losses & 3 & 0 & 1 & 5 & 5 & 5 & 2 & 2 & 23 \\
\hline & Draws & 2 & 0 & 2 & 0 & 0 & 0 & 3 & 0 & 7 \\
\hline & Difference & -2 & 6 & 2 & -4 & -4 & -4 & -1 & 2 & -5 \\
\hline & Rank & 3 & 1 & 2 & 5 & 5 & 4 & 3 & 3 & 5 \\
\hline \multirow[t]{5}{*}{$\mathrm{SBPSO}_{\mathrm{m} 6}$} & Wins & 1 & 4 & 2 & 2 & 3 & 3 & 1 & 5 & 21 \\
\hline & Losses & 3 & 1 & 1 & 2 & 2 & 2 & 2 & 1 & 14 \\
\hline & Draws & 2 & 1 & 3 & 2 & 1 & 1 & 3 & 0 & 13 \\
\hline & Difference & -2 & 3 & 1 & 0 & 1 & 1 & -1 & 4 & 7 \\
\hline & Rank & 3 & 2 & 3 & 4 & 3 & 2 & 3 & 2 & 3 \\
\hline \multirow[t]{5}{*}{$\mathrm{SBPSO}_{\mathrm{ACD}}$} & Wins & 4 & 4 & 1 & 4 & 5 & 5 & 5 & 6 & 34 \\
\hline & Losses & 2 & 1 & 2 & 1 & 1 & 0 & 0 & 0 & 7 \\
\hline & Draws & 0 & 1 & 3 & 1 & 0 & 1 & 1 & 0 & 7 \\
\hline & Difference & 2 & 3 & -1 & 3 & 4 & 5 & 5 & 6 & 27 \\
\hline & Rank & 2 & 2 & 4 & 2 & 2 & 1 & 1 & 1 & 1 \\
\hline
\end{tabular}




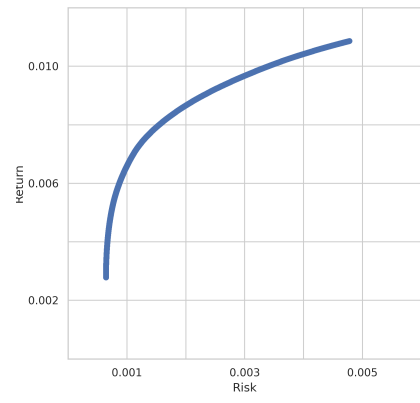

(a) True POF

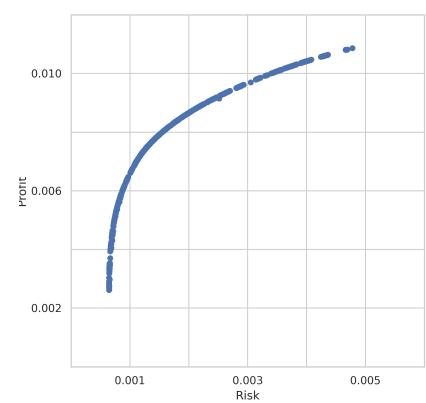

(d) GA

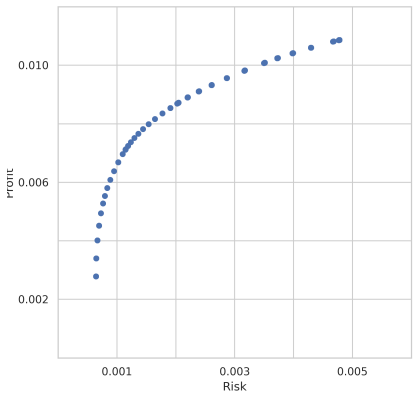

(b) $\mathrm{ABC}$

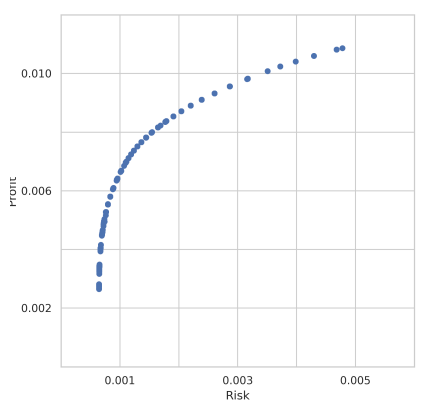

(e) PSO

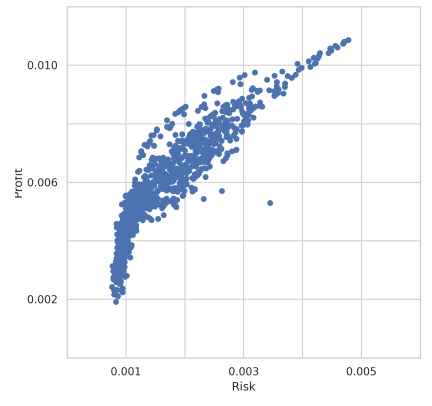

(c) FA

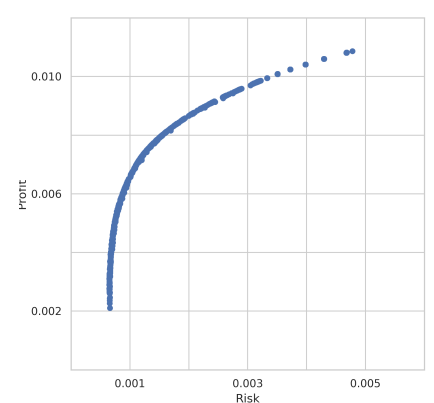

(f) SBPSO

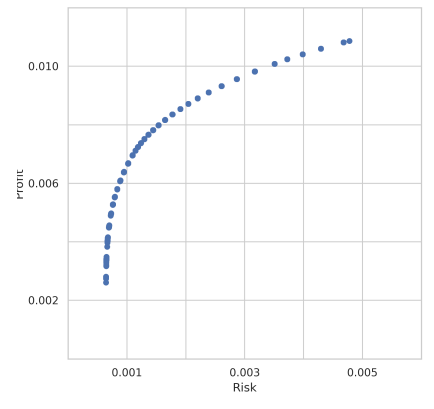

(g) $\mathrm{SBPSO}_{\mathrm{m} 6}$

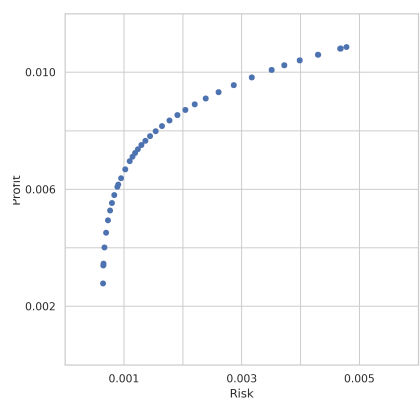

(h) $\mathrm{SBPSO}_{\mathrm{ACD}}$

Figure 6.11: Obtained Pareto-optimal fronts for Hang Seng

\subsubsection{DAX 100}

For the DAX 100 problem, $\mathrm{SBPSO}_{\mathrm{m} 6}$ and $\mathrm{SBPSO}_{\mathrm{ACD}}$ were the best and second best performing algorithms, respectively, as shown in Table 6.12. $\mathrm{SBPSO}_{\mathrm{m} 6}$ ranked second in the IGD and HV measures, beating all other algorithms except for ABC. Similarly, SBPSO $_{\mathrm{ACD}}$ ranked first for the GD measure. In comparison, the original SBPSO ranked second last for all the Pareto optimality measures. Table 6.11 shows that $\mathrm{SBPSO}_{\mathrm{m} 6}$ was extremely fast in comparison with the other algorithms. SBPSO $\mathrm{ACD}_{\mathrm{B}}$ was slightly slower than SBPSO, but still faster than ABC, FA, PSO and GA. Table 6.11 also shows that the average GD scores of $\mathrm{SBPSO}_{\mathrm{m} 6}$ and $\mathrm{SBPSO}_{\mathrm{ACD}}$ were an order of magnitude better 
CHAPTER 6. SET-BASED PARTICLE SWARM OPTIMIZATION IMPROVEMENT AND DIVERSITY ANALYSIS

than SBPSO. A visual analysis of the obtained POFs in Figure 6.12 shows that $\mathrm{SBPSO}_{\mathrm{m} 6}$ and $\mathrm{SBPSO}_{\mathrm{ACD}}$ were able to approximate the true POF as well as $\mathrm{ABC}$ and better than the PSO, FA, GA and original SBPSO.

Table 6.11: DAX 100 results for each performance measure

\begin{tabular}{lccccccccc}
\hline Algorithm & Result & $\mathbf{N}$ & $K$ & $R$ & $\bar{\sigma}$ & GD & IGD & HV & Time \\
\hline ABC & $\bar{x}$ & 49 & 14 & 0.008633 & 0.001219 & 0.000228 & 0.000304 & 0.787923 & 43.68 \\
& $\sigma$ & 1 & 1 & 0.000021 & 0.000029 & 0.000033 & 0.000000 & 0.000000 & 0.56 \\
\hline FA & $\bar{x}$ & 34 & 11 & 0.004485 & 0.000768 & 0.003702 & 0.000568 & 0.844798 & 166.26 \\
& $\sigma$ & 7 & 2 & 0.000606 & 0.000143 & 0.000571 & 0.000103 & 0.033878 & 21.28 \\
\hline GA & $\bar{x}$ & 39 & 10 & 0.008387 & 0.000953 & 0.000295 & 0.000307 & 0.893540 & 50.86 \\
& $\sigma$ & 1 & 0 & 0.000034 & 0.000025 & 0.000152 & 0.000004 & 0.057716 & 2.02 \\
\hline PSO & $\bar{x}$ & 42 & 5 & 0.008456 & 0.000967 & 0.000222 & 0.000304 & 0.816574 & 52.40 \\
& $\sigma$ & 1 & 0 & 0.000017 & 0.000013 & 0.000053 & 0.000001 & 0.057681 & 1.73 \\
\hline SBPSO & $\bar{x}$ & 34 & 6 & 0.007670 & 0.000960 & 0.002334 & 0.000357 & 0.826267 & 26.87 \\
& $\sigma$ & 5 & 1 & 0.000319 & 0.000105 & 0.000527 & 0.000019 & 0.054478 & 1.63 \\
\hline SBPSO $_{\mathrm{m} 6}$ & $\bar{x}$ & 43 & 4 & 0.008469 & 0.000972 & 0.000250 & 0.000306 & 0.878138 & 8.99 \\
& $\sigma$ & 0 & 0 & 0.000006 & 0.000000 & 0.000061 & 0.000008 & 0.065429 & 3.19 \\
\hline SBPSO $_{\mathrm{ACD}}$ & $\bar{x}$ & 42 & 4 & 0.008460 & 0.000971 & 0.000209 & 0.000346 & 0.804378 & 28.09 \\
& $\sigma$ & 9 & 0 & 0.000001 & 0.000000 & 0.000000 & 0.000000 & 0.165428 & 45.21 \\
\hline
\end{tabular}


CHAPTER 6. SET-BASED PARTICLE SWARM OPTIMIZATION IMPROVEMENT AND DIVERSITY ANALYSIS

Table 6.12: DAX 100 rankings for each performance measure

\begin{tabular}{|c|c|c|c|c|c|c|c|c|c|c|}
\hline Algorithm & Result & $\mathbf{N}$ & $K$ & $R$ & $\bar{\sigma}$ & GD & IGD & HV & Time & Overall \\
\hline \multirow[t]{5}{*}{$\mathrm{ABC}$} & Wins & 6 & 0 & 6 & 0 & 3 & 5 & 0 & 3 & 23 \\
\hline & Losses & 0 & 6 & 0 & 6 & 1 & 0 & 6 & 3 & 22 \\
\hline & Draws & 0 & 0 & 0 & 0 & 2 & 1 & 0 & 0 & 3 \\
\hline & Difference & 6 & -6 & 6 & -6 & 2 & 5 & -6 & 0 & 1 \\
\hline & Rank & 1 & 6 & 1 & 7 & 3 & 1 & 5 & 4 & 4 \\
\hline \multirow[t]{5}{*}{ FA } & Wins & 0 & 1 & 0 & 6 & 0 & 0 & 3 & 0 & 10 \\
\hline & Losses & 5 & 5 & 6 & 0 & 6 & 6 & 2 & 6 & 36 \\
\hline & Draws & 1 & 0 & 0 & 0 & 0 & 0 & 1 & 0 & 2 \\
\hline & Difference & -5 & -4 & -6 & 6 & -6 & -6 & 1 & -6 & -26 \\
\hline & Rank & 5 & 5 & 6 & 1 & 7 & 6 & 3 & 7 & 7 \\
\hline \multirow[t]{5}{*}{ GA } & Wins & 2 & 2 & 2 & 4 & 2 & 3 & 6 & 2 & 23 \\
\hline & Losses & 4 & 4 & 4 & 1 & 4 & 2 & 0 & 4 & 23 \\
\hline & Draws & 0 & 0 & 0 & 1 & 0 & 1 & 0 & 0 & 2 \\
\hline & Difference & -2 & -2 & -2 & 3 & -2 & 1 & 6 & -2 & 0 \\
\hline & Rank & 4 & 4 & 4 & 2 & 5 & 3 & 1 & 5 & 5 \\
\hline \multirow[t]{5}{*}{ PSO } & Wins & 3 & 4 & 3 & 3 & 4 & 4 & 1 & 1 & 23 \\
\hline & Losses & 2 & 2 & 2 & 2 & 0 & 1 & 2 & 5 & 16 \\
\hline & Draws & 1 & 0 & 1 & 1 & 2 & 1 & 3 & 0 & 9 \\
\hline & Difference & 1 & 2 & 1 & 1 & 4 & 3 & -1 & -4 & 7 \\
\hline & Rank & 3 & 2 & 3 & 3 & 2 & 2 & 4 & 6 & 3 \\
\hline \multirow[t]{5}{*}{ SBPSO } & Wins & 0 & 3 & 1 & 1 & 1 & 1 & 1 & 5 & 13 \\
\hline & Losses & 5 & 3 & 5 & 1 & 5 & 5 & 2 & 1 & 27 \\
\hline & Draws & 1 & 0 & 0 & 4 & 0 & 0 & 3 & 0 & 8 \\
\hline & Difference & -5 & 0 & -4 & 0 & -4 & -4 & -1 & 4 & -14 \\
\hline & Rank & 5 & 3 & 5 & 4 & 6 & 5 & 4 & 2 & 6 \\
\hline \multirow[t]{5}{*}{$\mathrm{SBPSO}_{\mathrm{m} 6}$} & Wins & 5 & 5 & 5 & 1 & 3 & 3 & 3 & 6 & 31 \\
\hline & Losses & 1 & 0 & 1 & 4 & 2 & 0 & 1 & 0 & 9 \\
\hline & Draws & 0 & 1 & 0 & 1 & 1 & 3 & 2 & 0 & 8 \\
\hline & Difference & 4 & 5 & 4 & -3 & 1 & 3 & 2 & 6 & 22 \\
\hline & Rank & 2 & 1 & 2 & 6 & 4 & 2 & 2 & 1 & 1 \\
\hline \multirow[t]{5}{*}{$\mathrm{SBPSO}_{\mathrm{ACD}}$} & Wins & 3 & 5 & 3 & 2 & 5 & 2 & 1 & 4 & 25 \\
\hline & Losses & 2 & 0 & 2 & 3 & 0 & 4 & 2 & 2 & 15 \\
\hline & Draws & 1 & 1 & 1 & 1 & 1 & 0 & 3 & 0 & 8 \\
\hline & Difference & 1 & 5 & 1 & -1 & 5 & -2 & -1 & 2 & 10 \\
\hline & Rank & 3 & 1 & 3 & 5 & 1 & 4 & 4 & 3 & 2 \\
\hline
\end{tabular}




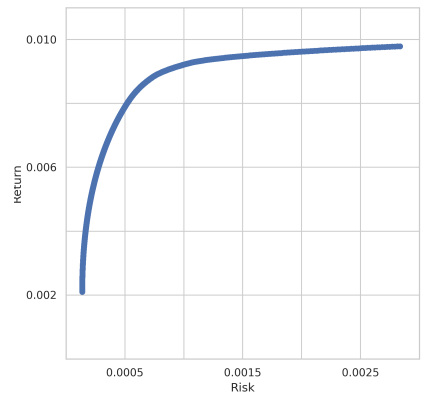

(a) True POF

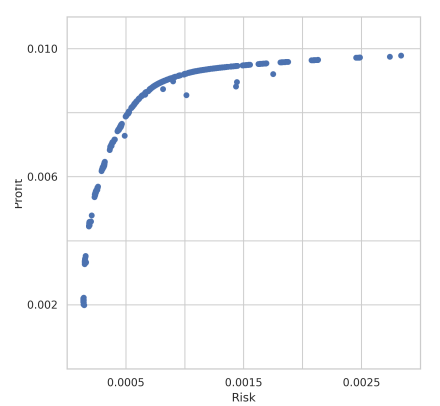

(d) GA

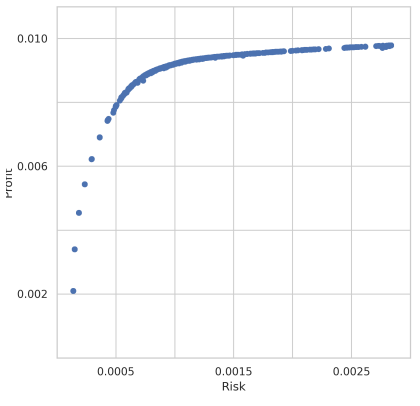

(b) $\mathrm{ABC}$

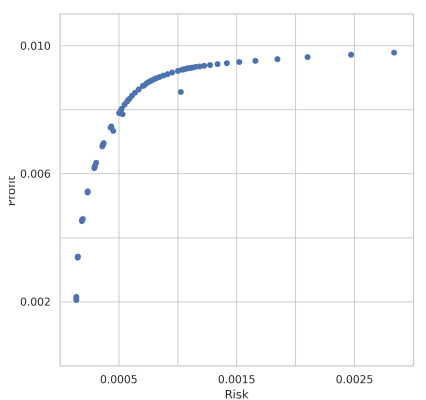

(e) PSO

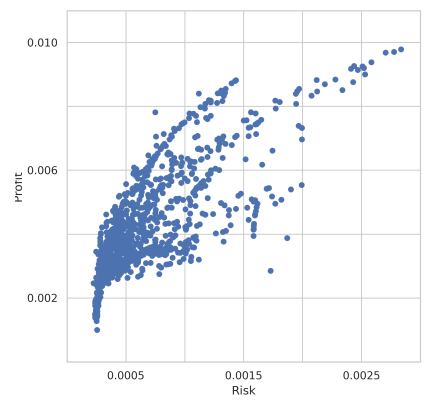

(c) FA

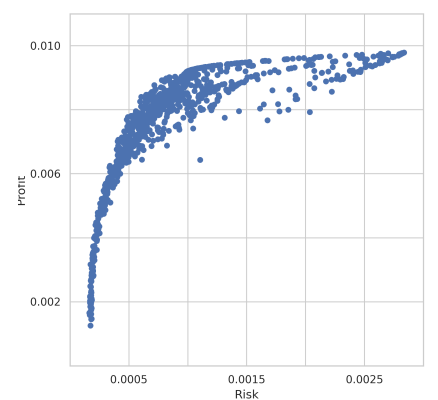

(f) SBPSO

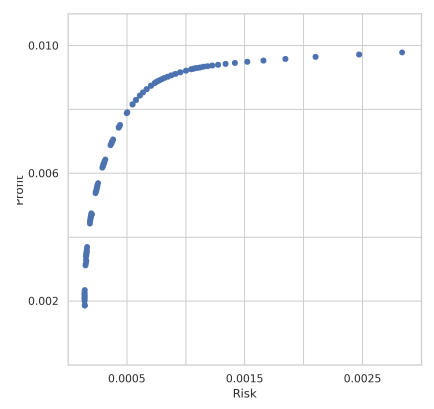

(g) $\mathrm{SBPSO}_{\mathrm{m} 6}$

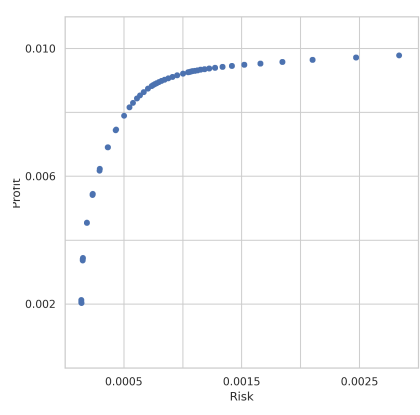

(h) $\mathrm{SBPSO}_{\mathrm{ACD}}$

Figure 6.12: Obtained Pareto-optimal fronts for DAX 100

\subsubsection{FTSE 100}

Table 6.13 show that the averages and standard deviations of the results obtained by $\mathrm{SBPSO}_{\mathrm{m} 6}$ were better than those of SBPSO. Likewise, $\mathrm{SBPSO}_{\mathrm{ACD}}$ also obtained results that were on average better than SBPSO with lower standard deviations, except for $\mathrm{HV}$ and time. $\mathrm{SBPSO}_{\mathrm{ACD}}$, on average, obtained portfolios that were slightly more profitable than PSO with slightly less risk. $\mathrm{SBPSO}_{\mathrm{m} 6}$ was also competitive with regard to risk and return as it obtained portfolios that were on average more profitable than FA, GA, PSO, SBPSO and $\mathrm{SBPSO}_{\mathrm{ACD}}$ at a similar level of risk. Table 6.14 shows that, as for the previous benchmark problem, $\mathrm{SBPSO}_{\mathrm{m} 6}$ ranked first overall and $\mathrm{SBPSO}_{\mathrm{ACD}}$ 
ranked second overall. However, $\mathrm{SBPSO}_{\mathrm{ACD}}$ ranked first in risk, GD and IGD. In Chapter 4 the FA ranked first for risk and last for GD and IGD. Therefore, $\mathrm{SBPSO}_{\mathrm{ACD}}$ is very competitive in that it can obtain solutions as close to the true POF that are extremely risk averse. $\mathrm{SBPSO}_{\mathrm{m} 6}$ generally ranked second and third for the performance measures, but ranked first for time and the size of the solutions obtained. The improvements in performance made by $\mathrm{SBPSO}_{\mathrm{ACD}}$ and $\mathrm{SBPSO}_{\mathrm{m} 6}$ over SBPSO can also been seen in the obtained POFs shown in Figure 6.13. $\mathrm{SBPSO}_{\mathrm{ACD}}$ and $\mathrm{SBPSO}_{\mathrm{m} 6}$ obtained POFs that closely resemble the true POF, outperforming the FA, GA, PSO and SBPSO. In comparison, the original SBPSO was the second-worst algorithm with regard to approximating the true POF.

Table 6.13: FTSE 100 results for each performance measure

\begin{tabular}{lccccccccc}
\hline Algorithm & Result & $\mathbf{N}$ & $K$ & $R$ & $\bar{\sigma}$ & GD & IGD & HV & Time \\
\hline ABC & $\bar{x}$ & 49 & 16 & 0.007277 & 0.001073 & 0.000166 & 0.000232 & 0.679689 & 47.09 \\
& $\sigma$ & 1 & 1 & 0.000011 & 0.000005 & 0.000020 & 0.000000 & 0.020228 & 1.02 \\
\hline FA & $\bar{x}$ & 36 & 12 & 0.004745 & 0.000779 & 0.003347 & 0.000467 & 0.714994 & 186.79 \\
& $\sigma$ & 4 & 2 & 0.000445 & 0.000098 & 0.000356 & 0.000087 & 0.013245 & 25.15 \\
\hline GA & $\bar{x}$ & 29 & 12 & 0.006635 & 0.000778 & 0.000408 & 0.000238 & 0.766213 & 58.45 \\
& $\sigma$ & 1 & 1 & 0.000163 & 0.000029 & 0.000321 & 0.000024 & 0.035444 & 1.29 \\
\hline PSO & $\bar{x}$ & 29 & 6 & 0.006667 & 0.000782 & 0.000190 & 0.000232 & 0.704657 & 59.86 \\
& $\sigma$ & 0 & 0 & 0.000008 & 0.000001 & 0.000039 & 0.000001 & 0.049243 & 0.95 \\
\hline SBPSO & $\bar{x}$ & 33 & 6 & 0.006605 & 0.000822 & 0.001206 & 0.000264 & 0.725351 & 13.81 \\
& $\sigma$ & 6 & 2 & 0.000176 & 0.000061 & 0.000472 & 0.000017 & 0.037266 & 5.43 \\
\hline SBPSO $_{\mathrm{m} 6}$ & $\bar{x}$ & 30 & 5 & 0.006683 & 0.000785 & 0.000247 & 0.000235 & 0.741994 & 10.57 \\
& $\sigma$ & 0 & 0 & 0.000007 & 0.000001 & 0.000038 & 0.000007 & 0.049170 & 3.55 \\
\hline SBPSO $_{\mathrm{ACD}}$ & $\bar{x}$ & 29 & 5 & 0.006655 & 0.000775 & 0.000169 & 0.000232 & 0.711504 & 35.75 \\
& $\sigma$ & 1 & 0 & 0.000024 & 0.000011 & 0.000019 & 0.000001 & 0.051801 & 61.04 \\
\hline
\end{tabular}


CHAPTER 6. SET-BASED PARTICLE SWARM OPTIMIZATION IMPROVEMENT AND DIVERSITY ANALYSIS

Table 6.14: FTSE 100 rankings for each performance measure

\begin{tabular}{|c|c|c|c|c|c|c|c|c|c|c|}
\hline Algorithm & Result & $\mathbf{N}$ & $K$ & $R$ & $\bar{\sigma}$ & GD & IGD & HV & Time & Overall \\
\hline \multirow[t]{5}{*}{$\mathrm{ABC}$} & Wins & 6 & 0 & 6 & 0 & 5 & 4 & 0 & 3 & 24 \\
\hline & Losses & 0 & 6 & 0 & 6 & 0 & 1 & 5 & 3 & 21 \\
\hline & Draws & 0 & 0 & 0 & 0 & 1 & 1 & 1 & 0 & 3 \\
\hline & Difference & 6 & -6 & 6 & -6 & 5 & 3 & -5 & 0 & 3 \\
\hline & Rank & 1 & 4 & 1 & 5 & 1 & 2 & 6 & 4 & 3 \\
\hline \multirow[t]{5}{*}{ FA } & Wins & 5 & 1 & 0 & 2 & 0 & 0 & 3 & 0 & 11 \\
\hline & Losses & 1 & 4 & 6 & 0 & 6 & 6 & 3 & 6 & 32 \\
\hline & Draws & 0 & 1 & 0 & 4 & 0 & 0 & 0 & 0 & 5 \\
\hline & Difference & 4 & -3 & -6 & 2 & -6 & -6 & 0 & -6 & -21 \\
\hline & Rank & 2 & 3 & 6 & 2 & 6 & 5 & 3 & 7 & 7 \\
\hline \multirow[t]{5}{*}{ GA } & Wins & 0 & 1 & 1 & 2 & 2 & 2 & 6 & 2 & 16 \\
\hline & Losses & 4 & 4 & 2 & 0 & 4 & 3 & 0 & 4 & 21 \\
\hline & Draws & 2 & 1 & 3 & 4 & 0 & 1 & 0 & 0 & 11 \\
\hline & Difference & -4 & -3 & -1 & 2 & -2 & -1 & 6 & -2 & -5 \\
\hline & Rank & 4 & 3 & 4 & 2 & 4 & 3 & 1 & 5 & 6 \\
\hline \multirow[t]{5}{*}{ PSO } & Wins & 0 & 3 & 2 & 3 & 4 & 4 & 0 & 1 & 17 \\
\hline & Losses & 4 & 3 & 2 & 1 & 2 & 1 & 2 & 5 & 20 \\
\hline & Draws & 2 & 0 & 2 & 2 & 0 & 1 & 4 & 0 & 11 \\
\hline & Difference & -4 & 0 & 0 & 2 & 2 & 3 & -2 & -4 & -3 \\
\hline & Rank & 4 & 2 & 3 & 2 & 2 & 2 & 5 & 6 & 4 \\
\hline \multirow[t]{5}{*}{ SBPSO } & Wins & 3 & 4 & 1 & 1 & 1 & 1 & 2 & 5 & 18 \\
\hline & Losses & 2 & 0 & 2 & 5 & 5 & 5 & 2 & 1 & 22 \\
\hline & Draws & 1 & 2 & 3 & 0 & 0 & 0 & 2 & 0 & 8 \\
\hline & Difference & 1 & 4 & -1 & -4 & -4 & -4 & 0 & 4 & -4 \\
\hline & Rank & 3 & 1 & 4 & 4 & 5 & 4 & 3 & 2 & 5 \\
\hline \multirow[t]{5}{*}{$\mathrm{SBPSO}_{\mathrm{m} 6}$} & Wins & 3 & 4 & 5 & 2 & 3 & 2 & 3 & 6 & 28 \\
\hline & Losses & 2 & 0 & 1 & 2 & 3 & 3 & 1 & 0 & 12 \\
\hline & Draws & 1 & 2 & 0 & 2 & 0 & 1 & 2 & 0 & 8 \\
\hline & Difference & 1 & 4 & 4 & 0 & 0 & -1 & 2 & 6 & 16 \\
\hline & Rank & 3 & 1 & 2 & 3 & 3 & 3 & 2 & 1 & 1 \\
\hline \multirow[t]{5}{*}{$\mathrm{SBPSO}_{\mathrm{ACD}}$} & Wins & 0 & 4 & 1 & 4 & 5 & 6 & 1 & 4 & 25 \\
\hline & Losses & 4 & 0 & 3 & 0 & 0 & 0 & 2 & 2 & 11 \\
\hline & Draws & 2 & 2 & 2 & 2 & 1 & 0 & 3 & 0 & 12 \\
\hline & Difference & -4 & 4 & -2 & 4 & 5 & 6 & -1 & 2 & 14 \\
\hline & Rank & 4 & 1 & 5 & 1 & 1 & 1 & 4 & 3 & 2 \\
\hline
\end{tabular}




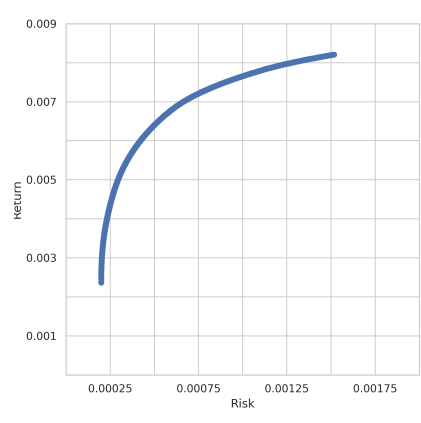

(a) True POF

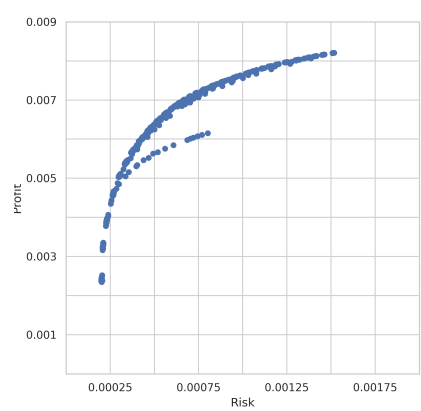

(d) GA

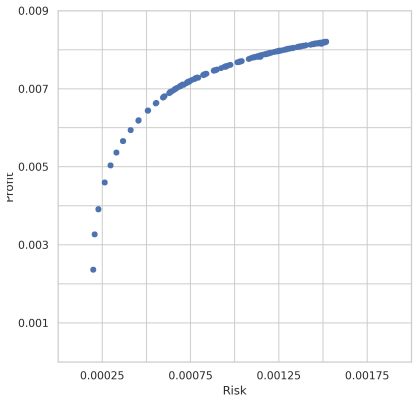

(b) $\mathrm{ABC}$

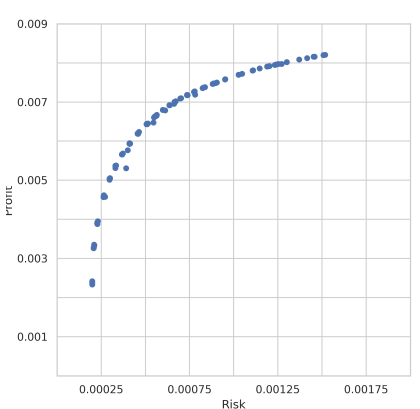

(e) PSO

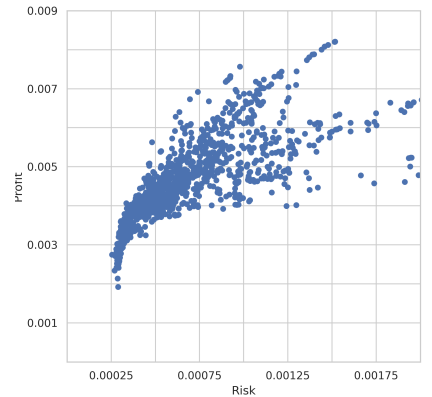

(c) FA

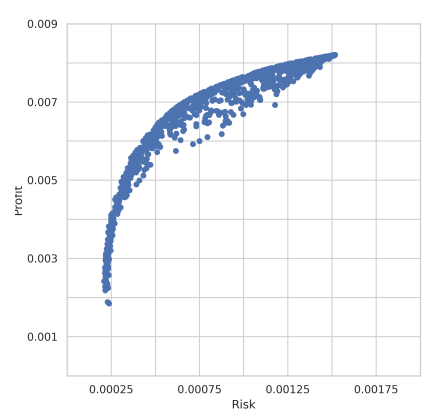

(f) SBPSO

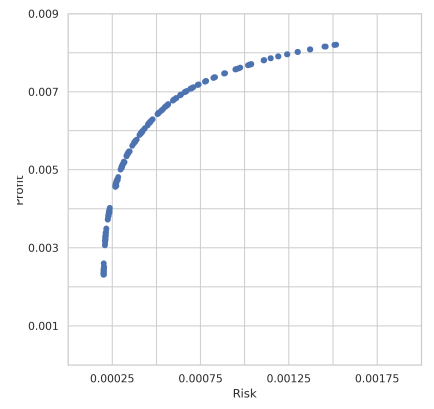

(g) $\mathrm{SBPSO}_{\mathrm{m} 6}$

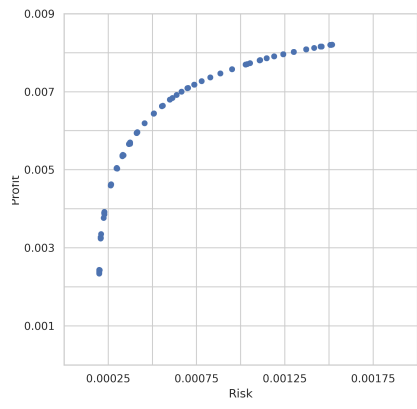

(h) $\mathrm{SBPSO}_{\mathrm{ACD}}$

Figure 6.13: Obtained Pareto-optimal fronts for FTSE 100

\subsubsection{S\&P 100}

This benchmark problem was particularly difficult for the original SBPSO as discussed in Section 6.5.3. Table 6.15 shows that $\mathrm{SBPSO}_{\mathrm{m} 6}$ and $\mathrm{SBPSO}_{\mathrm{ACD}}$ made significant gains in performance over the original SBPSO. Specifically, $\mathrm{SBPSO}_{\mathrm{m} 6}$ and $\mathrm{SBPSO}_{\mathrm{ACD}}$ on average obtained more profitable portfolios than SBPSO at a similar level of risk. Furthermore, these portfolios were significantly closer to the true POF, as indicated by the lower GD and IGD scores. Table 6.16 shows that $\mathrm{SBPSO}_{\mathrm{ACD}}$ ranked first for all three Pareto optimality measures and ranked first overall. However, the increase in performance made by $\mathrm{SBPSO}_{\mathrm{ACD}}$ is at the cost of a longer time to reach the stopping condi- 
CHAPTER 6. SET-BASED PARTICLE SWARM OPTIMIZATION IMPROVEMENT AND DIVERSITY ANALYSIS

tion. $\mathrm{SBPSO}_{\mathrm{ACD}}$ ranked second last, behind the $\mathrm{FA}$, with regard to time to reach the stopping condition. In contrast, the original SBPSO ranked second in time. $\mathrm{SBPSO}_{\mathrm{m} 6}$ did not rank as well as $\mathrm{SBPSO}_{\mathrm{ACD}}, \mathrm{ABC}$ or PSO for GD and IGD, but did obtain similar average values while being statistically significantly faster. Furthermore, $\mathrm{SBPSO}_{\mathrm{m} 6}$ ranked second overall. Lastly, Figure 6.14 shows that $\mathrm{SBPSO}_{\mathrm{m} 6}$ and $\mathrm{SBPSO}_{\mathrm{ACD}}$ were able to approximate the true POF much better than the original SBPSO and better than the GA, FA and PSO.

\subsection{5}

Table 6.15: S\&P 100 results for each performance measure

\begin{tabular}{lccccccccc}
\hline Algorithm & Result & $\mathbf{N}$ & $K$ & $R$ & $\bar{\sigma}$ & GD & IGD & HV & Time \\
\hline ABC & $\bar{x}$ & 49 & 24 & 0.007843 & 0.001398 & 0.000236 & 0.000241 & 0.841527 & 56.54 \\
& $\sigma$ & 1 & 3 & 0.000031 & 0.000035 & 0.000031 & 0.000000 & 0.018891 & 1.21 \\
\hline FA & $\bar{x}$ & 38 & 18 & 0.005203 & 0.000891 & 0.003536 & 0.000535 & 0.825091 & 230.75 \\
& $\sigma$ & 5 & 2 & 0.000691 & 0.000246 & 0.000453 & 0.000093 & 0.016919 & 24.39 \\
\hline GA & $\bar{x}$ & 35 & 6 & 0.007430 & 0.001177 & 0.000810 & 0.000260 & 0.881914 & 54.52 \\
& $\sigma$ & 2 & 0 & 0.000088 & 0.000052 & 0.000265 & 0.000007 & 0.042699 & 1.31 \\
\hline PSO & $\bar{x}$ & 44 & 8 & 0.007705 & 0.001245 & 0.000269 & 0.000241 & 0.892730 & 64.55 \\
& $\sigma$ & 1 & 0 & 0.000010 & 0.000012 & 0.000096 & 0.000001 & 0.052169 & 1.26 \\
\hline SBPSO & $\bar{x}$ & 43 & 9 & 0.007269 & 0.001219 & 0.001775 & 0.000301 & 0.890120 & 35.69 \\
& $\sigma$ & 2 & 3 & 0.000428 & 0.000174 & 0.000620 & 0.000036 & 0.031804 & 5.56 \\
\hline SBPSO $_{\text {m6 }}$ & $\bar{x}$ & 44 & 6 & 0.007716 & 0.001248 & 0.000275 & 0.000247 & 0.875057 & 16.45 \\
& $\sigma$ & 0 & 0 & 0.000006 & 0.000001 & 0.000044 & 0.000003 & 0.044175 & 2.78 \\
\hline SBPSO $_{\mathrm{ACD}}$ & $\bar{x}$ & 44 & 6 & 0.007708 & 0.001244 & 0.000205 & 0.000242 & 0.872137 & 91.53 \\
& $\sigma$ & 0 & 0 & 0.000001 & 0.000000 & 0.000022 & 0.000001 & 0.049381 & 166.15 \\
\hline
\end{tabular}


CHAPTER 6. SET-BASED PARTICLE SWARM OPTIMIZATION IMPROVEMENT AND DIVERSITY ANALYSIS

Table 6.16: S\&P 100 rankings for each performance measure

\begin{tabular}{|c|c|c|c|c|c|c|c|c|c|c|}
\hline Algorithm & Result & $\mathbf{N}$ & $K$ & $R$ & $\bar{\sigma}$ & GD & IGD & HV & Time & Overall \\
\hline \multirow[t]{5}{*}{$\mathrm{ABC}$} & Wins & 6 & 0 & 6 & 0 & 4 & 4 & 1 & 3 & 24 \\
\hline & Losses & 0 & 6 & 0 & 6 & 1 & 0 & 5 & 3 & 21 \\
\hline & Draws & 0 & 0 & 0 & 0 & 1 & 2 & 0 & 0 & 3 \\
\hline & Difference & 6 & -6 & 6 & -6 & 3 & 4 & -4 & 0 & 3 \\
\hline & Rank & 1 & 6 & 1 & 7 & 2 & 1 & 2 & 4 & 4 \\
\hline \multirow[t]{5}{*}{ FA } & Wins & 1 & 1 & 0 & 6 & 0 & 0 & 0 & 0 & 8 \\
\hline & Losses & 5 & 5 & 6 & 0 & 6 & 6 & 6 & 6 & 40 \\
\hline & Draws & 0 & 0 & 0 & 0 & 0 & 0 & 0 & 0 & 0 \\
\hline & Difference & -4 & -4 & -6 & 6 & -6 & -6 & -6 & -6 & -32 \\
\hline & Rank & 4 & 5 & 5 & 1 & 6 & 5 & 3 & 7 & 7 \\
\hline \multirow[t]{5}{*}{ GA } & Wins & 0 & 6 & 1 & 5 & 2 & 2 & 2 & 4 & 22 \\
\hline & Losses & 6 & 0 & 4 & 1 & 4 & 4 & 0 & 2 & 21 \\
\hline & Draws & 0 & 0 & 1 & 0 & 0 & 0 & 4 & 0 & 5 \\
\hline & Difference & -6 & 6 & -3 & 4 & -2 & -2 & 2 & 2 & 1 \\
\hline & Rank & 5 & 1 & 4 & 2 & 4 & 3 & 1 & 3 & 5 \\
\hline \multirow[t]{5}{*}{ PSO } & Wins & 3 & 3 & 3 & 2 & 4 & 4 & 2 & 2 & 23 \\
\hline & Losses & 1 & 3 & 2 & 4 & 1 & 0 & 0 & 4 & 15 \\
\hline & Draws & 2 & 0 & 1 & 0 & 1 & 2 & 4 & 0 & 10 \\
\hline & Difference & 2 & 0 & 1 & -2 & 3 & 4 & 2 & -2 & 8 \\
\hline & Rank & 2 & 3 & 3 & 5 & 2 & 1 & 1 & 5 & 3 \\
\hline \multirow[t]{5}{*}{ SBPSO } & Wins & 2 & 2 & 1 & 4 & 1 & 1 & 2 & 5 & 18 \\
\hline & Losses & 4 & 4 & 4 & 2 & 5 & 5 & 0 & 1 & 25 \\
\hline & Draws & 0 & 0 & 1 & 0 & 0 & 0 & 4 & 0 & 5 \\
\hline & Difference & -2 & -2 & -3 & 2 & -4 & -4 & 2 & 4 & -7 \\
\hline & Rank & 3 & 4 & 4 & 3 & 5 & 4 & 1 & 2 & 6 \\
\hline \multirow[t]{5}{*}{$\mathrm{SBPSO}_{\mathrm{m} 6}$} & Wins & 3 & 4 & 5 & 1 & 3 & 3 & 2 & 6 & 27 \\
\hline & Losses & 1 & 1 & 1 & 5 & 3 & 3 & 0 & 0 & 14 \\
\hline & Draws & 2 & 1 & 0 & 0 & 0 & 0 & 4 & 0 & 7 \\
\hline & Difference & 2 & 3 & 4 & -4 & 0 & 0 & 2 & 6 & 13 \\
\hline & Rank & 2 & 2 & 2 & 6 & 3 & 2 & 1 & 1 & 2 \\
\hline \multirow[t]{5}{*}{$\mathrm{SBPSO}_{\mathrm{ACD}}$} & Wins & 3 & 4 & 3 & 3 & 6 & 4 & 2 & 1 & 26 \\
\hline & Losses & 1 & 1 & 2 & 3 & 0 & 0 & 0 & 5 & 12 \\
\hline & Draws & 2 & 1 & 1 & 0 & 0 & 2 & 4 & 0 & 10 \\
\hline & Difference & 2 & 3 & 1 & 0 & 6 & 4 & 2 & -4 & 14 \\
\hline & Rank & 2 & 2 & 3 & 4 & 1 & 1 & 1 & 6 & 1 \\
\hline
\end{tabular}




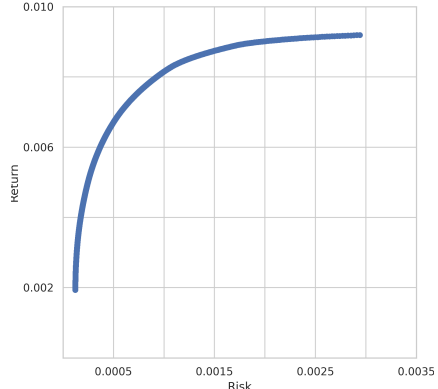

(a) True POF

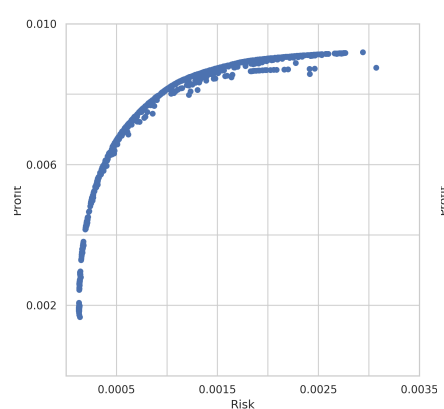

(d) GA

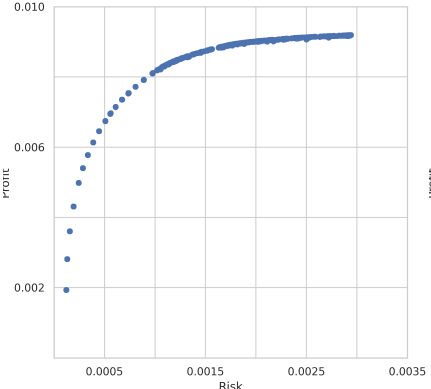

(b) $\mathrm{ABC}$

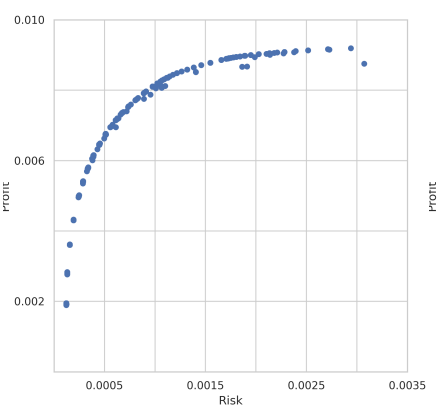

(e) PSO

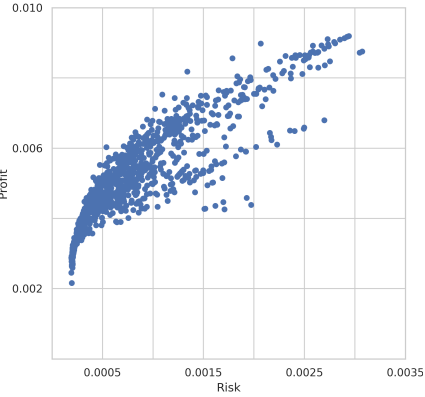

(c) FA

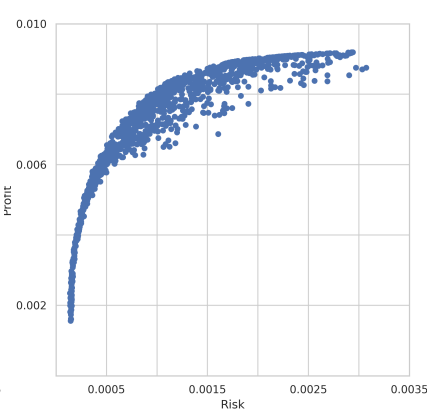

(f) SBPSO

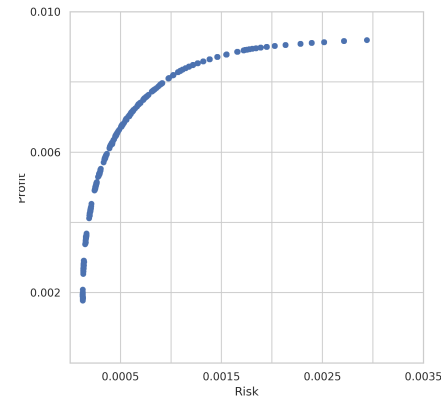

(g) $\mathrm{SBPSO}_{\mathrm{m}}$

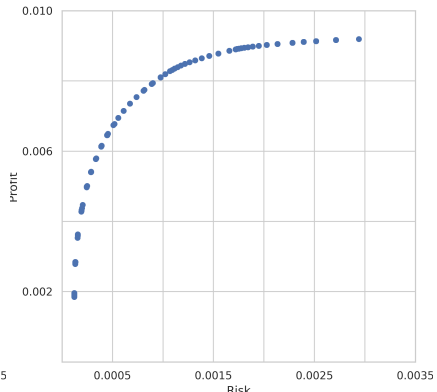

(h) $\mathrm{SBPSO}_{\mathrm{ACD}}$

Figure 6.14: Obtained Pareto-optimal fronts for S\&P 100

\subsubsection{Nikkei 225}

Table 6.17 shows that $\mathrm{SBPSO}_{\mathrm{m} 6}$ and $\mathrm{SBPSO}_{\mathrm{ACD}}$ were very competitive with regard to risk and return. The portfolios obtained by $\mathrm{SBPSO}_{\mathrm{m} 6}$ and $\mathrm{SBPSO}_{\mathrm{ACD}}$ were more profitable than the FA, GA, PSO and SBPSO, while being less risky. Furthermore, $\mathrm{SBPSO}_{\mathrm{m} 6}$ and $\mathrm{SBPSO}_{\mathrm{ACD}}$ ranked first and second overall, respectively, as shown in Table 6.18. In comparison, the original SBPSO, despite being the fastest algorithm to reach the stopping condition, ranked second last. $\mathrm{SBPSO}_{\mathrm{ACD}}$ was statistically significantly better than the other algorithms for the GD and IGD measure. However, $\mathrm{SBPSO}_{\mathrm{ACD}}$ was statistically significantly slower than the other algorithms to reach the stopping condition 
CHAPTER 6. SET-BASED PARTICLE SWARM OPTIMIZATION IMPROVEMENT AND DIVERSITY ANALYSIS

except for the FA. In contrast, $\mathrm{SBPSO}_{\mathrm{m} 6}$ was the second fastest algorithm to reach the stopping condition and ranked second and fourth for the GD and IGD measures, respectively. In addition to the high rankings of $\mathrm{SBPSO}_{\mathrm{m} 6}$ and $\mathrm{SBPSO}_{\mathrm{ACD}}$, Figure 6.15 shows that $\mathrm{SBPSO}_{\mathrm{m}}$ and $\mathrm{SBPSO}_{\mathrm{ACD}}$ were the only algorithms to approximate the true POF - a significant gain in performance over the original approach.

Table 6.17: Nikkei 225 results for each performance measure

\begin{tabular}{lccccccccc}
\hline Algorithm & Result & $\mathbf{N}$ & $K$ & $R$ & $\bar{\sigma}$ & GD & IGD & HV & Time \\
\hline ABC & $\bar{x}$ & 49 & 24 & 0.003348 & 0.000858 & 0.000470 & 0.000210 & 0.924789 & 254.99 \\
& $\sigma$ & 0 & 6 & 0.000022 & 0.000015 & 0.000221 & 0.000001 & 0.046806 & 2.91 \\
\hline FA & $\bar{x}$ & 28 & 12 & 0.001086 & 0.000962 & 0.004191 & 0.000490 & 0.734680 & 1087.28 \\
& $\sigma$ & 6 & 7 & 0.000484 & 0.000114 & 0.000561 & 0.000056 & 0.064953 & 117.14 \\
\hline GA & $\bar{x}$ & 46 & 22 & 0.003185 & 0.000858 & 0.001702 & 0.000276 & 0.906150 & 248.82 \\
& $\sigma$ & 4 & 3 & 0.000105 & 0.000068 & 0.000344 & 0.000019 & 0.043527 & 2.82 \\
\hline PSO & $\bar{x}$ & 44 & 7 & 0.003264 & 0.000782 & 0.000703 & 0.000218 & 0.957354 & 266.89 \\
& $\sigma$ & 2 & 0 & 0.000023 & 0.000022 & 0.000126 & 0.000008 & 0.037560 & 4.40 \\
\hline SBPSO & $\bar{x}$ & 42 & 10 & 0.002454 & 0.000800 & 0.002396 & 0.000353 & 0.826649 & 29.22 \\
& $\sigma$ & 4 & 5 & 0.000592 & 0.000065 & 0.000535 & 0.000065 & 0.067572 & 4.19 \\
\hline SBPSO $_{\text {m6 }}$ & $\bar{x}$ & 44 & 4 & 0.003310 & 0.000794 & 0.000260 & 0.000223 & 0.916014 & 63.19 \\
& $\sigma$ & 0 & 0 & 0.000007 & 0.000001 & 0.000060 & 0.000005 & 0.047097 & 6.13 \\
\hline SBPSO $_{\text {ACD }}$ & $\bar{x}$ & 44 & 4 & 0.003305 & 0.000791 & 0.000139 & 0.000209 & 0.898166 & 342.28 \\
& $\sigma$ & 0 & 0 & 0.000001 & 0.000001 & 0.000027 & 0.000001 & 0.024326 & 59.95 \\
\hline
\end{tabular}


CHAPTER 6. SET-BASED PARTICLE SWARM OPTIMIZATION IMPROVEMENT AND DIVERSITY ANALYSIS

Table 6.18: Nikkei 225 rankings for each performance measure

\begin{tabular}{|c|c|c|c|c|c|c|c|c|c|c|}
\hline Algorithm & Result & $\mathbf{N}$ & $K$ & $R$ & $\bar{\sigma}$ & GD & IGD & HV & Time & Overall \\
\hline \multirow[t]{5}{*}{$\mathrm{ABC}$} & Wins & 6 & 0 & 6 & 1 & 4 & 5 & 3 & 3 & 28 \\
\hline & Losses & 0 & 6 & 0 & 4 & 2 & 1 & 0 & 3 & 16 \\
\hline & Draws & 0 & 0 & 0 & 1 & 0 & 0 & 3 & 0 & 4 \\
\hline & Difference & 6 & -6 & 6 & -3 & 2 & 4 & 3 & 0 & 12 \\
\hline & Rank & 1 & 5 & 1 & 4 & 3 & 2 & 2 & 4 & 3 \\
\hline \multirow[t]{5}{*}{ FA } & Wins & 0 & 2 & 0 & 0 & 0 & 0 & 0 & 0 & 2 \\
\hline & Losses & 6 & 3 & 6 & 6 & 6 & 6 & 6 & 6 & 45 \\
\hline & Draws & 0 & 1 & 0 & 0 & 0 & 0 & 0 & 0 & 1 \\
\hline & Difference & -6 & -1 & -6 & -6 & -6 & -6 & -6 & -6 & -43 \\
\hline & Rank & 6 & 3 & 7 & 5 & 7 & 7 & 7 & 7 & 7 \\
\hline \multirow[t]{5}{*}{ GA } & Wins & 5 & 1 & 2 & 1 & 2 & 2 & 3 & 4 & 20 \\
\hline & Losses & 1 & 5 & 4 & 4 & 4 & 4 & 1 & 2 & 25 \\
\hline & Draws & 0 & 0 & 0 & 1 & 0 & 0 & 2 & 0 & 3 \\
\hline & Difference & 4 & -4 & -2 & -3 & -2 & -2 & 2 & 2 & -5 \\
\hline & Rank & 2 & 4 & 5 & 4 & 5 & 5 & 3 & 3 & 5 \\
\hline \multirow[t]{5}{*}{ PSO } & Wins & 1 & 4 & 3 & 5 & 3 & 4 & 5 & 2 & 27 \\
\hline & Losses & 2 & 2 & 3 & 0 & 3 & 2 & 0 & 4 & 16 \\
\hline & Draws & 3 & 0 & 0 & 1 & 0 & 0 & 1 & 0 & 5 \\
\hline & Difference & -1 & 2 & 0 & 5 & 0 & 2 & 5 & -2 & 11 \\
\hline & Rank & 4 & 2 & 4 & 1 & 4 & 3 & 1 & 5 & 4 \\
\hline \multirow[t]{5}{*}{ SBPSO } & Wins & 1 & 2 & 1 & 3 & 1 & 1 & 1 & 6 & 16 \\
\hline & Losses & 4 & 3 & 5 & 0 & 5 & 5 & 5 & 0 & 27 \\
\hline & Draws & 1 & 1 & 0 & 3 & 0 & 0 & 0 & 0 & 5 \\
\hline & Difference & -3 & -1 & -4 & 3 & -4 & -4 & -4 & 6 & -11 \\
\hline & Rank & 5 & 3 & 6 & 2 & 6 & 6 & 6 & 1 & 6 \\
\hline \multirow[t]{5}{*}{$\mathrm{SBPSO}_{\mathrm{m} 6}$} & Wins & 3 & 5 & 5 & 3 & 5 & 3 & 2 & 5 & 31 \\
\hline & Losses & 2 & 0 & 1 & 2 & 1 & 3 & 1 & 1 & 11 \\
\hline & Draws & 1 & 1 & 0 & 1 & 0 & 0 & 3 & 0 & 6 \\
\hline & Difference & 1 & 5 & 4 & 1 & 4 & 0 & 1 & 4 & 20 \\
\hline & Rank & 3 & 1 & 2 & 3 & 2 & 4 & 4 & 2 & 1 \\
\hline \multirow[t]{5}{*}{$\mathrm{SBPSO}_{\mathrm{ACD}}$} & Wins & 2 & 5 & 4 & 4 & 6 & 6 & 2 & 1 & 30 \\
\hline & Losses & 3 & 0 & 2 & 1 & 0 & 0 & 3 & 5 & 14 \\
\hline & Draws & 1 & 1 & 0 & 1 & 0 & 0 & 1 & 0 & 4 \\
\hline & Difference & -1 & 5 & 2 & 3 & 6 & 6 & -1 & -4 & 16 \\
\hline & Rank & 4 & 1 & 3 & 2 & 1 & 1 & 5 & 6 & 2 \\
\hline
\end{tabular}




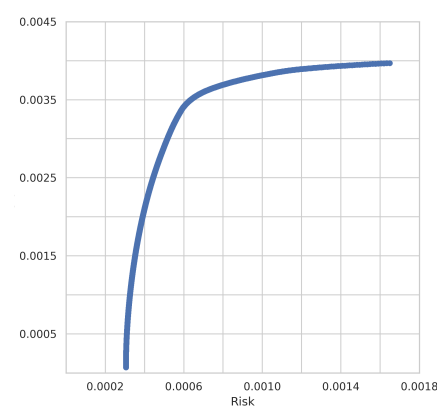

(a) True POF

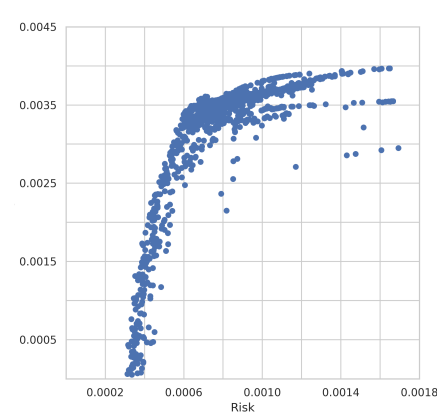

(d) GA

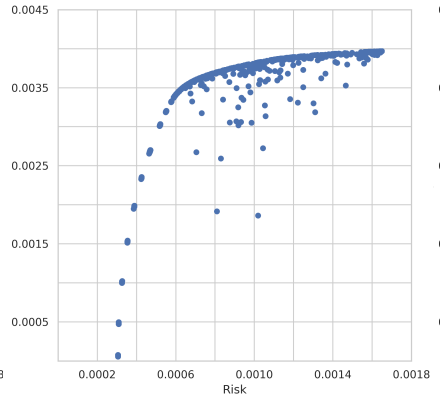

(b) $\mathrm{ABC}$

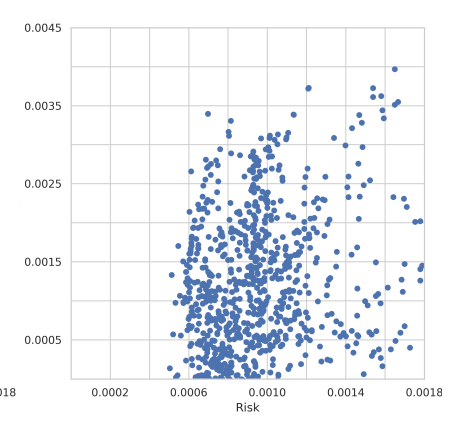

(c) FA

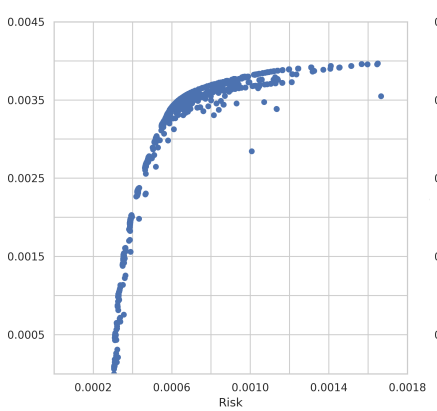

(e) PSO

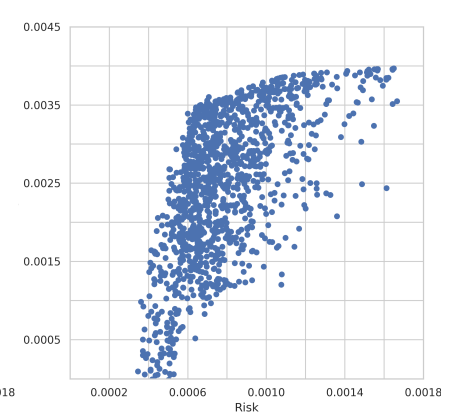

(f) SBPSO

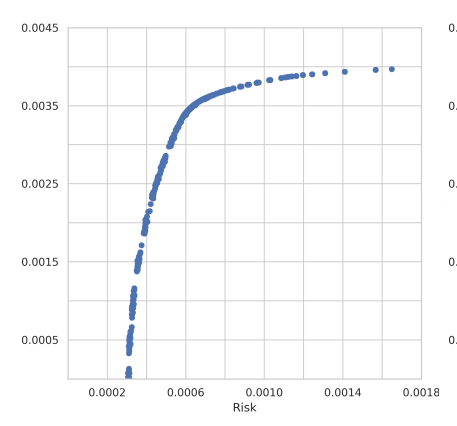

(g) $\mathrm{SBPSO}_{\mathrm{m} 6}$

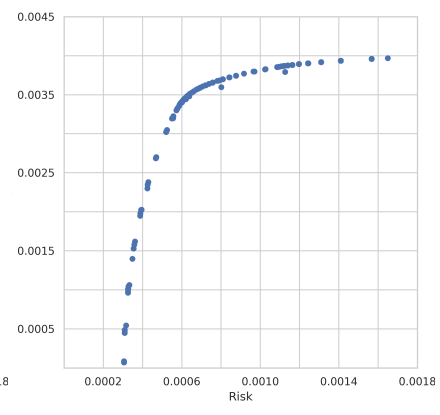

(h) $\mathrm{SBPSO}_{\mathrm{ACD}}$

Figure 6.15: Obtained Pareto-optimal fronts for Nikkei 225

\subsection{Summary}

It was hypothesized at the end of Chapter 4 that the gap in performance between set-based particle swarm optimization (SBPSO) the other algorithms, namely artificial bee colony (ABC), genetic algorithm (GA), and particle swarm optimization ( $\mathrm{PSO}$ ), was due to an inadequate weight determinate stage and an imbalance in exploration and exploitation behaviour. An in depth look into the diversity and performance of SBPSO confirmed these hypotheses. In doing so, several SBPSO modifications were studied. The modification to allow the weight optimizer to execute until there was no improvement in solution quality, instead of a fixed number of time steps, resulted in significant gains 
CHAPTER 6. SET-BASED PARTICLE SWARM OPTIMIZATION

in performance. The imbalance in exploration and exploitation behaviour was corrected with the introduction of an exploration balance coefficient. The modfied SBPSO with a completely deterministic weight optimizer using adaptive coordinate descent (ACD) was also investigated. Performance analysis showed that ACD variant improved the closeness of the found portfolios to the true POFs, but at the cost of a longer search time. Finally, the performance of SBPSO with the modifications and the ACD variant was evaluated against the ABC, firefly algorithm (FA), GA, PSO, and original SBPSO, as seen in Chapter 4. The results showed that new SBPSO approaches outperformed the other algorithms. 


\section{Chapter 7}

\section{Set-based Particle Swarm Optimization for Multi-objective Portfolio Optimization}

Portfolio optimization, by definition, is a MOOP with risk and profit, or some form of the two, as competing objectives. Single-objective portfolio optimization requires a trade-off coefficient to be specified in order to balance the two objectives. A collection of equally good, but different, solutions can then be obtained by solving the single-objective optimization problem for various trade-off coefficient values. A more sophisticated and appropriate approach would be to use a MOOP solving algorithm to identify an equally spread set of non-dominated solutions. This chapter ${ }^{1}$ proposes the first multi-objective optimization (MOO) approach to SBPSO. Other approaches to MOOPs for sets by Khan and Engelbrecht used a fuzzy aggregation operator, namely the unified And-Or operator, to convert a multi-objective problem formulation into a fuzzy single-objective formulation [98. The proposed MOO approach to SBPSO does not require the use of an aggregation operator, but rather optimizes the multiple objectives independently. Furthermore, the proposed approach makes use of the improvements to SBPSO for portfolio optimization identified in the previous chapter. Thus, assets are selected that maximize return and minimize risk. Weight determination for the assets is also a MOOP for which MGPSO (refer to Chapter 2.3) is used. For optimal performance, the parameters of MGPSO need to be tuned for portfolio optimization. However, parameter tuning is often an expensive and time-consuming process. An approach to randomly sample control parameter values for MGPSO that satisfy stability conditions is also investigated in this chapter.

The remainder of this chapter is organized as follows: Section 7.1 investi-

\footnotetext{
${ }^{1}$ This chapter is currently being reviewed for the IEEE Swarm Intelligence Symposium under the title "A tuning free approach to multi-guide particle swarm optimization" [55] and the journal IEEE Transactions on Evolutionary Computation under the title "Multi-guide set-based particle swarm optimization for portfolio optimization" [57.
} 
gates the approach of randomly sampling control parameter values that satisfy stability conditions for MGPSO. Section 7.2 proposes multi-guide set-based particle swarm optimization (MGSBPSO) for portfolio optimization. The empirical process used to assess the proposed approach is given in Section 7.3. The results are discussed in Section 7.4 and the findings are summarised in Section 7.5 ,

\subsection{Tuning-free Approach to Multi-guide Particle Swarm Optimization}

This section proposes a tuning-free approach to MGPSO that uses control parameter values sampled from the stability region defined in equation (2.8). Section 7.1.1 proposes the tuning-free approach to MGPSO. The experimental process and results empirically validating the tuning-free approach are presented in Sections 7.1 .2 and 7.1 .3 respectively.

\subsubsection{Proposal}

A formal control parameter performance study for MGPSO showed that its performance is sensitive to its control parameters 152. Furthermore, Carolus and Engelbrecht showed that the inertia component value has the greatest sensitivity and is the most important control parameter to tune when optimizing the MGPSO [16]. Equation (2.8) defines the theoretical stability conditions of the MGPSO control parameters to guarantee order- 1 and order- 2 stability. If the control parameter values satisfy the stability conditions, the swarm is guaranteed to reach a state of equilibrium where the velocity of each particle is zero. The proposed approach is to then randomly sample control parameter values for the MGPSO from the stability region at every iteration for every particle. Since each particle samples its own values for the control parameters, a mix of search behaviours will be used by the swarms. If the randomly generated control parameter values do not satisfy the MGPSO stability conditions of equation (2.8), then they are discarded and new control parameter values are generated. This process is repeated until control parameter values that satisfy equation (2.8) are generated. However, to avoid MGPSO from spending too much time on generating parameter values, the process is limited to ten attempts. If no parameter values generated satisfy the stability conditions, the current parameters are reused. Therefore, this is a tuning-free approach to MGPSO with a guarantee that the swarm will reach a state of equilibrium. It is expected that the tuning-free approach, referred to as stable MGPSO or MGPSO $_{\mathrm{s}}$ for short, will perform similarly to the MGPSO using tuned control parameter values. 


\subsubsection{Empirical Process}

The WFG tool kit (described in Appendix E) was used to test if $\mathrm{MGPSO}_{\mathrm{s}}$ performs similarly to MGPSO using tuned parameter values, listed in Table 7.1. The WFG tool kit can be used to generate scalable MOOPs. In this study, two-objective and three-objective benchmark problems were used with 10, 30 and 50 dimensions. The jMetal framework ${ }^{2}$ provided POFs used to measure the GD, IGD and HV scores for each algorithm. These scores were recorded over 30 independent runs, where each independent run lasted 2000 iterations. The archive of each MGPSO algorithm at the end of each independent run was regarded as the obtained POF for that independent run. The MGPSO algorithms were implemented and executed using the CIlib framework 141. Lastly, $\mathrm{MGPSO}_{\mathrm{s}}$ used the same tournament sizes as MGPSO to ensure that any differences in performance between the two were a direct result of the parameter value generation.

Table 7.1: MGPSO control parameters used for each WFG test problem

\begin{tabular}{|c|c|c|c|c|c|c|c|c|c|}
\hline 30.2751 .651 .801 .75 Problem & Objectives & $\left|S_{1}\right|$ & $\left|S_{2}\right|$ & $\left|S_{3}\right|$ & $T$ & $w$ & $c_{1}$ & $c_{2}$ & $c_{3}$ \\
\hline WFG1 & 2 & 45 & 5 & & 3 & 0.275 & 1.65 & 1.75 & 1.05 \\
\hline WFG2 & 2 & 24 & 26 & & 2 & 0.750 & 1.15 & 1.70 & 1.05 \\
\hline WFG3 & 2 & 31 & 19 & & 2 & 0.600 & 1.60 & 1.85 & 0.95 \\
\hline WFG4 & 2 & 2 & 48 & & 2 & 0.100 & 0.80 & 1.65 & 1.70 \\
\hline WFG5 & 2 & 50 & 0 & & 2 & 0.600 & 0.80 & 1.60 & 1.85 \\
\hline WFG6 & 2 & 19 & 31 & & 2 & 0.525 & 0.65 & 0.60 & 1.65 \\
\hline WFG7 & 2 & 29 & 21 & & 2 & 0.450 & 1.20 & 1.85 & 1.55 \\
\hline WFG8 & 2 & 37 & 13 & & 3 & 0.750 & 1.00 & 1.65 & 1.05 \\
\hline WFG9 & 2 & 13 & 37 & & 2 & 0.275 & 1.00 & 0.50 & 1.70 \\
\hline WFG1 & 3 & 37 & 4 & 9 & 2 & 0.125 & 1.20 & 1.30 & 1.75 \\
\hline WFG2 & 3 & 24 & 25 & 1 & 2 & 0.275 & 1.25 & 1.40 & 1.70 \\
\hline WFG3 & 3 & 29 & 10 & 11 & 2 & 0.525 & 1.65 & 1.75 & 0.75 \\
\hline WFG4 & 3 & 29 & 21 & 0 & 2 & 0.275 & 1.75 & 0.50 & 1.05 \\
\hline WFG5 & 3 & 2 & 48 & 0 & 3 & 0.575 & 0.60 & 1.85 & 1.75 \\
\hline WFG6 & 3 & 5 & 30 & 15 & 3 & 0.300 & 0.90 & 0.90 & 1.90 \\
\hline WFG7 & 3 & 10 & 22 & 18 & 2 & 0.425 & 1.45 & 1.50 & 1.40 \\
\hline WFG8 & 3 & 4 & 23 & 23 & 3 & 0.425 & 0.95 & 1.75 & 1.85 \\
\hline WFG9 & 3 & 4 & 45 & 1 & 2 & 0.275 & 1.25 & 0.75 & 1.50 \\
\hline
\end{tabular}

\subsubsection{Results}

Table 7.2 shows that the average scores between the two approaches for all two-objective WFG problems were very similar, with marginal differences. Likewise, Table 7.3 shows that the algorithms performed very similarly. There was no case where the performance between either algorithm differ by an order of magnitude. Furthermore, performance was consistent as the dimensionality of the problems increased. Table 7.4 shows that there was no statistical significant difference between the MGPSO with tuned parameters and $\mathrm{MGPSO}_{\mathrm{s}}$

\footnotetext{
${ }^{2}$ https://github.com/jMetal/jMetal
} 
with regard to GD and IGD for all two-objective WFG problems. A similar result was observed in Table 7.5 where there was no statistical significant difference between the IGD scores of the two algorithms for three-objective WFG problems. MGPSO performed better on three-objective WFG3 with regard to GD for all dimensions, otherwise there was no statistically meaningful difference in the three-objective WFG GD results. The HV scores, however, were not as cut and dry. For the 10 dimensional two-objective WFG problems, MGPSO $_{\mathrm{s}}$ scored more wins than the tuned MGPSO. However, the number of wins by $\mathrm{MGPSO}_{\mathrm{s}}$ deteriorated as the dimensionality increased. Nevertheless, there were cases where $\mathrm{MGPSO}_{\mathrm{s}}$ scored higher $\mathrm{HV}$ values on average, such as the 50 dimensional WFG2 and WFG6 problems. For the three-objective WFG HV results, the algorithms reached a draw for more problems than for the two-objective WFG HV results, but MGPSO still has the advantage. Overall, the results show that the performance of MGPSO with control parameter values randomly sampled from the stability region defined in equation (2.8) was similar to that of the MGPSO using tuned parameters. Furthermore, $\mathrm{MGPSO}_{\mathrm{s}}$ did not require computationally expensive control parameter tuning unlike MGPSO.

Table 7.2: MGPSO versus $\mathrm{MGPSO}_{\mathrm{s}}$ : Mean results for 2-objective WFG

\begin{tabular}{|c|c|c|c|c|c|c|c|c|c|c|}
\hline & & \multicolumn{3}{|c|}{$10 d$} & \multicolumn{3}{|c|}{ 30d } & \multicolumn{3}{|c|}{$50 d$} \\
\hline & & GD & IGD & HV & GD & IGD & HV & GD & IGD & HV \\
\hline \multirow[t]{2}{*}{ WFG1 } & MGPSO & 0.4874 & 0.0930 & 4.7760 & 0.4912 & 0.0930 & 1.9958 & 0.4912 & 0.0930 & 1.8051 \\
\hline & MGPSO $_{\mathrm{s}}$ & 0.4866 & 0.0930 & 5.8049 & 0.4915 & 0.0930 & 2.0912 & 0.4908 & 0.0930 & 1.9070 \\
\hline \multirow[t]{2}{*}{ WFG2 } & MGPSO & 0.4844 & 0.1200 & 6.1056 & 0.4849 & 0.1199 & 5.6518 & 0.4849 & 0.1199 & 5.3721 \\
\hline & MGPSO $_{\mathrm{s}}$ & 0.4845 & 0.1200 & 6.0987 & 0.4846 & 0.1200 & 6.0069 & 0.4848 & 0.1200 & 5.8025 \\
\hline \multirow[t]{2}{*}{ WFG3 } & MGPSO & 0.4860 & 0.0927 & 5.5900 & 0.4863 & 0.0927 & 5.1302 & 0.4865 & 0.0927 & 4.7155 \\
\hline & $\mathrm{MGPSO}_{\mathrm{s}}$ & 0.4860 & 0.0927 & 5.5930 & 0.4862 & 0.0927 & 5.3485 & 0.4863 & 0.0927 & 5.0490 \\
\hline \multirow[t]{2}{*}{ WFG4 } & MGPSO & 0.4847 & 0.0923 & 3.0780 & 0.4848 & 0.0922 & 2.7094 & 0.4849 & 0.0922 & 2.5551 \\
\hline & MGPSO $_{\mathrm{s}}$ & 0.4847 & 0.0922 & 3.0084 & 0.4850 & 0.0922 & 2.5778 & 0.4850 & 0.0922 & 2.3641 \\
\hline \multirow[t]{2}{*}{ WFG5 } & MGPSO & 0.4846 & 0.0922 & 2.9002 & 0.4846 & 0.0922 & 2.8856 & 0.4846 & 0.0922 & 2.8741 \\
\hline & $\mathrm{MGPSO}_{\mathrm{s}}$ & 0.4846 & 0.0922 & 2.9177 & 0.4847 & 0.0922 & 2.9076 & 0.4845 & 0.0923 & 2.8987 \\
\hline \multirow[t]{2}{*}{ WFG6 } & MGPSO & 0.4846 & 0.0922 & 3.0825 & 0.4848 & 0.0922 & 2.7441 & 0.4849 & 0.0922 & 2.5826 \\
\hline & MGPSO $_{\mathrm{s}}$ & 0.4846 & 0.0922 & 3.1527 & 0.4847 & 0.0922 & 3.0301 & 0.4847 & 0.0922 & 2.8607 \\
\hline \multirow[t]{2}{*}{ WFG7 } & MGPSO & 0.4846 & 0.0923 & 3.3350 & 0.4846 & 0.0923 & 3.2523 & 0.4847 & 0.0922 & 3.0524 \\
\hline & $\mathrm{MGPSO}_{\mathrm{s}}$ & 0.4845 & 0.0923 & 3.3359 & 0.4846 & 0.0923 & 3.2933 & 0.4847 & 0.0922 & 3.1298 \\
\hline \multirow[t]{2}{*}{ WFG8 } & MGPSO & 0.4863 & 0.0919 & 1.9608 & 0.4850 & 0.0921 & 2.2243 & 0.4850 & 0.0921 & 2.1365 \\
\hline & $\mathrm{MGPSO}_{\mathrm{s}}$ & 0.4900 & 0.0918 & 1.8354 & 0.4849 & 0.0921 & 2.2662 & 0.4849 & 0.0921 & 2.3031 \\
\hline \multirow[t]{2}{*}{ WFG9 } & MGPSO & 0.4846 & 0.0922 & 3.0987 & 0.4848 & 0.0922 & 2.7293 & 0.4849 & 0.0922 & 2.6676 \\
\hline & MGPSO $_{\mathrm{s}}$ & 0.4846 & 0.0922 & 3.1148 & 0.4849 & 0.0922 & 2.7660 & 0.4849 & 0.0922 & 2.6310 \\
\hline
\end{tabular}


CHAPTER 7. SET-BASED PARTICLE SWARM OPTIMIZATION FOR MULTI-OBJECTIVE PORTFOLIO OPTIMIZATION

Table 7.3: $\mathrm{MGPSO}$ versus $\mathrm{MGPSO}_{\mathrm{s}}$ : Mean results for 3-objective WFG

\begin{tabular}{|c|c|c|c|c|c|c|c|c|c|c|}
\hline & & \multicolumn{3}{|c|}{$10 d$} & \multicolumn{3}{|c|}{ 30d } & \multicolumn{3}{|c|}{$50 d$} \\
\hline & & GD & IGD & HV & GD & IGD & HV & GD & IGD & HV \\
\hline \multirow[t]{2}{*}{ WFG 1} & MGPSO & 0.4978 & 0.0821 & 21.2980 & 0.4992 & 0.0822 & 18.9183 & 0.4989 & 0.0822 & 18.8586 \\
\hline & $\mathrm{MGPSO}_{\mathrm{s}}$ & 0.4943 & 0.0822 & 27.0259 & 0.4986 & 0.0822 & 19.1579 & 0.4987 & 0.0822 & 18.7677 \\
\hline \multirow[t]{2}{*}{ WFG 2} & MGPSO & 0.4890 & 0.0692 & 54.6878 & 0.4886 & 0.0694 & 51.6535 & 0.4893 & 0.0693 & 49.4731 \\
\hline & $\mathrm{MGPSO}_{\mathrm{s}}$ & 0.4889 & 0.0693 & 53.6745 & 0.4884 & 0.0694 & 50.0681 & 0.4885 & 0.0695 & 46.9867 \\
\hline \multirow[t]{2}{*}{ WFG3 } & MGPSO & 0.5125 & 0.0371 & 6.1599 & 0.5116 & 0.0369 & 5.0631 & 0.5117 & 0.0368 & 4.6463 \\
\hline & $\mathrm{MGPSO}_{\mathrm{s}}$ & 0.5130 & 0.0372 & 6.4064 & 0.5125 & 0.0371 & 5.7365 & 0.5125 & 0.0371 & 5.2145 \\
\hline \multirow[t]{2}{*}{ WFG 4} & MGPSO & 0.4869 & 0.0371 & 29.0554 & 0.4871 & 0.0371 & 26.8826 & 0.4871 & 0.0370 & 25.7538 \\
\hline & $\mathrm{MGPSO}_{\mathrm{s}}$ & 0.4869 & 0.0371 & 26.8352 & 0.4870 & 0.0371 & 25.0841 & 0.4871 & 0.0371 & 24.8821 \\
\hline \multirow[t]{2}{*}{ WFG5 } & MGPSO & 0.4866 & 0.0364 & 27.8030 & 0.4867 & 0.0364 & 26.7786 & 0.4867 & 0.0363 & 26.4431 \\
\hline & $\mathrm{MGPSO}_{\mathrm{s}}$ & 0.4865 & 0.0364 & 28.6180 & 0.4866 & 0.0364 & 27.4528 & 0.4867 & 0.0364 & 27.0387 \\
\hline \multirow[t]{2}{*}{ WFG6 } & MGPSO & 0.4868 & 0.0365 & 30.2637 & 0.4870 & 0.0365 & 26.0443 & 0.4873 & 0.0365 & 24.6185 \\
\hline & $\mathrm{MGPSO}_{\mathrm{s}}$ & 0.4868 & 0.0365 & 30.4891 & 0.4868 & 0.0365 & 30.0679 & 0.4871 & 0.0365 & 27.6585 \\
\hline \multirow[t]{2}{*}{ WFG 7} & MGPSO & 0.4868 & 0.0365 & 31.7465 & 0.4869 & 0.0365 & 30.2471 & 0.4870 & 0.0365 & 28.4909 \\
\hline & $\mathrm{MGPSO}_{\mathrm{s}}$ & 0.4868 & 0.0365 & 31.9518 & 0.4869 & 0.0365 & 30.6151 & 0.4871 & 0.0365 & 29.4147 \\
\hline \multirow[t]{2}{*}{ WFG 8} & MGPSO & 0.4881 & 0.0362 & 21.4920 & 0.4876 & 0.0363 & 22.5010 & 0.4875 & 0.0363 & 22.0185 \\
\hline & $\mathrm{MGPSO}_{\mathrm{s}}$ & 0.4880 & 0.0362 & 21.2494 & 0.4875 & 0.0363 & 22.7724 & 0.4874 & 0.0363 & 22.2965 \\
\hline \multirow[t]{2}{*}{ WFG9 } & MGPSO & 0.4867 & 0.0363 & 27.9181 & 0.4868 & 0.0363 & 25.3823 & 0.4871 & 0.0363 & 24.6624 \\
\hline & $\mathrm{MGPSO}_{\mathrm{s}}$ & 0.4866 & 0.0363 & 27.4443 & 0.4867 & 0.0363 & 26.1049 & 0.4870 & 0.0363 & 25.4001 \\
\hline
\end{tabular}

Table 7.4: 2-objective WFG rankings for each performance measure

\begin{tabular}{|c|c|c|c|c|c|c|c|c|c|c|c|}
\hline \multirow[b]{2}{*}{ Algorithm } & \multirow[b]{2}{*}{ Result } & \multicolumn{3}{|c|}{$10 d$} & \multicolumn{3}{|c|}{$30 d$} & \multicolumn{3}{|c|}{$50 d$} & \multirow[b]{2}{*}{ Overall } \\
\hline & & GD & IGD & HV & GD & IGD & HV & GD & IGD & HV & \\
\hline \multirow{5}{*}{ MGPSO } & Wins & 0 & 0 & 3 & 0 & 0 & 6 & 0 & 0 & 7 & 16 \\
\hline & Losses & 0 & 0 & 3 & 0 & 0 & 1 & 0 & 0 & 1 & 5 \\
\hline & Draws & 9 & 9 & 3 & 9 & 9 & 2 & 9 & 9 & 1 & 60 \\
\hline & Difference & 0 & 0 & 0 & 0 & 0 & 5 & 0 & 0 & 6 & 11 \\
\hline & Rank & 1 & 1 & 1 & 1 & 1 & 1 & 1 & 1 & 1 & 1 \\
\hline \multirow[t]{5}{*}{$\mathrm{MGPSO}_{\mathrm{s}}$} & Wins & 0 & 0 & 3 & 0 & 0 & 1 & 0 & 0 & 1 & 5 \\
\hline & Losses & 0 & 0 & 3 & 0 & 0 & 6 & 0 & 0 & 7 & 16 \\
\hline & Draws & 9 & 9 & 3 & 9 & 9 & 2 & 9 & 9 & 1 & 60 \\
\hline & Difference & 0 & 0 & 0 & 0 & 0 & -5 & 0 & 0 & -6 & -11 \\
\hline & Rank & 1 & 1 & 1 & 1 & 1 & 2 & 1 & 1 & 2 & 2 \\
\hline
\end{tabular}


CHAPTER 7. SET-BASED PARTICLE SWARM OPTIMIZATION FOR MULTI-OBJECTIVE PORTFOLIO OPTIMIZATION

Table 7.5: 3-objective WFG rankings for each performance measure

\begin{tabular}{|c|c|c|c|c|c|c|c|c|c|c|c|}
\hline \multirow[b]{2}{*}{ Algorithm } & \multirow[b]{2}{*}{ Result } & \multicolumn{3}{|c|}{$10 d$} & \multicolumn{3}{|c|}{ 30d } & \multicolumn{3}{|c|}{$50 \mathrm{~d}$} & \multirow[b]{2}{*}{ Overall } \\
\hline & & GD & IGD & HV & GD & IGD & HV & GD & IGD & HV & \\
\hline \multirow{5}{*}{ MGPSO } & Wins & 0 & 0 & 4 & 0 & 0 & 6 & 0 & 0 & 4 & 14 \\
\hline & Losses & 0 & 0 & 2 & 0 & 0 & 2 & 0 & 0 & 1 & 5 \\
\hline & Draws & 9 & 9 & 3 & 9 & 9 & 1 & 9 & 9 & 4 & 62 \\
\hline & Difference & 0 & 0 & 2 & 0 & 0 & 4 & 0 & 0 & 3 & 9 \\
\hline & Rank & 1 & 1 & 1 & 1 & 1 & 1 & 1 & 1 & 1 & 1 \\
\hline \multirow[t]{5}{*}{$\mathrm{MGPSO}_{\mathrm{s}}$} & Wins & 0 & 0 & 2 & 0 & 0 & 2 & 0 & 0 & 1 & 5 \\
\hline & Losses & 0 & 0 & 4 & 0 & 0 & 6 & 0 & 0 & 4 & 14 \\
\hline & Draws & 9 & 9 & 3 & 9 & 9 & 1 & 9 & 9 & 4 & 62 \\
\hline & Difference & 0 & 0 & -2 & 0 & 0 & -4 & 0 & 0 & -3 & -9 \\
\hline & Rank & 1 & 1 & 2 & 1 & 1 & 2 & 1 & 1 & 2 & 2 \\
\hline
\end{tabular}

\subsection{A New Approach to Multi-objective Set-based Portfolio Optimization}

This section proposes a multi-objective set-based algorithm for multi-objective portfolio optimization. Elements from MGPSO are incorporated into SBPSO to enable SBPSO to solve multiple objectives simultaneously. The proposed approached, referred to as MGSBPSO uses multiple swarms, where each swarm optimizes one of the multiple objectives in the asset space. Thus, there is a swarm to select assets that minimize risk and a swarm to select assets that maximize profit. As for MGPSO, non-dominated solutions found by the swarms are stored in an archive (initially empty) of a fixed size. The archive management process as described in Section 2.3.2 is also used in the MGSBPSO. However, the crowding distance of the non-dominated solutions in the archive is calculated with respect to their objective function values instead of their set-based positions, as the set-based positions lack distance in the traditional sense. Non-dominated solutions are selected from the archive using tournament selection and are used to guide the particles to non-dominated regions of the search space. Like the archive management strategy, the crowding distance of the non-dominated solutions in the tournament is calculated with respect to their objective function values. Furthermore, the successful modifications identified in Chapter 6 are also included in the MGSBPSO, namely the removal of zero-weighted assets; the immediate calculation of the objective function for single-asset portfolios; the decision to allow the weight optimizer to execute until it converges; only allowing assets to be removed via the weight determination stage and the exploration balance coefficient for improved convergence behaviour. Taking these improvements into account as well as the 


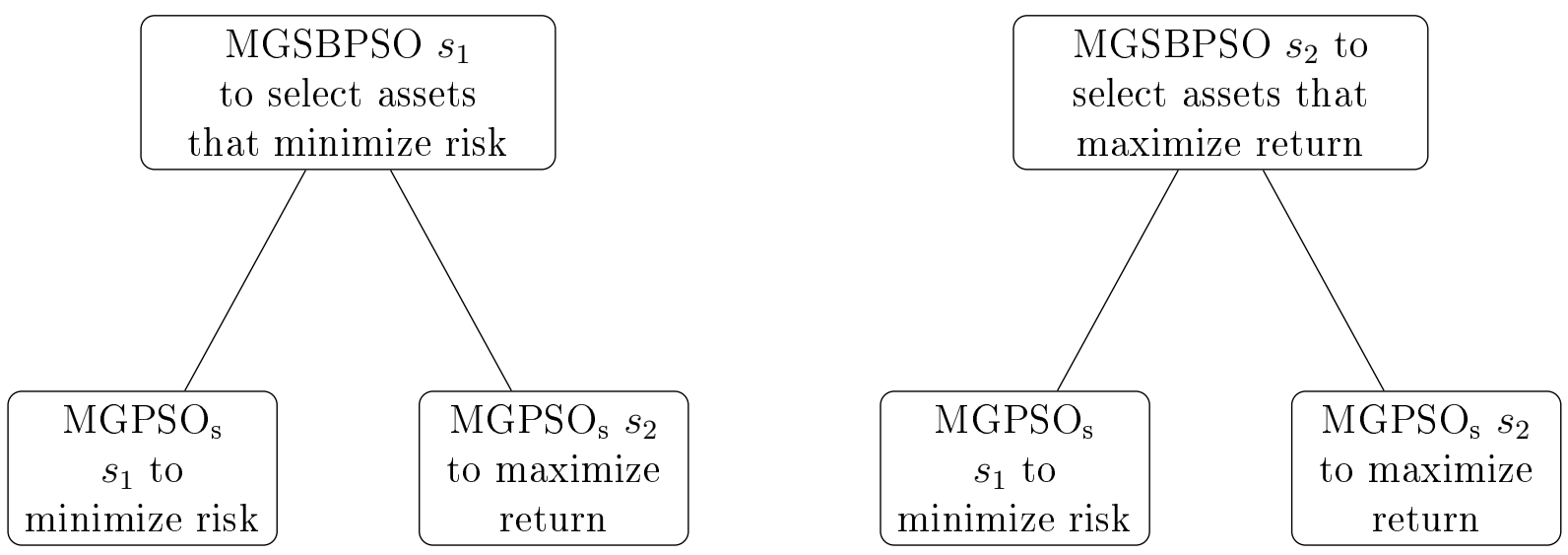

Figure 7.1: MGSBPSO Structure

archive guide, the velocity equation is

$$
\begin{aligned}
V_{i}(t+1)= & \lambda_{c}(t) r_{1}(t) \otimes\left(Y_{i}(t) \ominus X_{i}(t)\right) \\
& \oplus \lambda_{i} \lambda_{c}(t) r_{2}(t) \otimes\left(\hat{Y}_{i}(t) \ominus X_{i}(t)\right) \\
& \oplus\left(1-\lambda_{i}\right) \lambda_{c} r_{3}(t) \otimes\left(\hat{A}_{i}(t) \ominus X_{i}(t)\right) \\
& \oplus\left(1-\lambda_{c}(t)\right) r_{4}(t) \otimes A_{i}(t)
\end{aligned}
$$

where $r_{1}, r_{2}, r_{3}$ and $r_{4}$ are random values, each sampled from a uniform distribution in the range $[0,1] ; \lambda_{c}$ is the linearly increasing exploration balance coefficient; $X_{i}$ is the position of particle $i$; $Y_{i}$ is the best position found by particle $i ; \hat{Y}_{i}$ is the best position within particle $i$ 's neighbourhood; $\hat{A}_{i}$ is the archive guide for particle $i$; the influence of the archive guide is controlled by the archive coefficient $\lambda_{i} ; A_{i}$ is shorthand for $U \backslash(X(t) \cup Y(t) \cup \hat{Y}(t))$ where $U$ is the set universe, and $\otimes$ and $\oplus$ are the set-based operators defined in Section 2.4.

For the purpose of weight determination, the newly proposed $\mathrm{MGPSO}_{\mathrm{s}}$ is used. Hence, asset weight determination is also a multi-objective optimization task. For each set-particle, a MGPSO $\mathrm{M}_{\mathrm{s}}$ is instantiated to optimize the corresponding asset weights with regard to risk and return. Figure 7.1 illustrates the overall structure of the swarms in MGSBPSO and their objective.

Figure 7.1 shows that there are two $\mathrm{MGPSO}_{\mathrm{s}}$ algorithms, one to find continuous valued solutions for each set-swarm in MGSBPSO. Each $\mathrm{MGPSO}_{\mathrm{s}}$ has its own archive. Specifically, the $\mathrm{MGPSO}_{\mathrm{s}}$ for MGSBPSO $s_{1}$ (that maximizes return) has its own archive and the $\mathrm{MGPSO}_{\mathrm{s}}$ for MGSBPSO $s_{2}$ (that minimizes risk) has its own archive. There is also a global archive, the MGSBPSO archive, which is used to store the best solutions found by the MGPSO $\mathrm{S}_{\mathrm{s}}$ algorithms. An $\mathrm{MGPSO}_{\mathrm{s}}$ terminates when no non-dominated solutions are added to their archive over three iterations. The non-dominated solutions in the archive of an $\mathrm{MGPSO}_{\mathrm{s}}$ are then inserted into the global archive along with the corresponding set-position. Lastly, the best objective function value of the 
non-dominated solutions in an $\mathrm{MGPSO}_{\mathrm{s}}$ archive is assigned to the corresponding set-particle with regard to the objective of the swarm the set-particle is in. For example, if the set-particle is in the swarm to minimize risk, then the best risk value of the non-dominated solutions in the $\mathrm{MGPSO}_{\mathrm{s}}$ archive is used. Algorithm 12 presents pseudocode for the multi-objective weight optimization process and how the MGPSO $_{\mathrm{s}}$ archives interact with the global archive. The pseudocode for MGSBPSO is given in Algorithm 13 .

$\overline{\text { Algorithm } 12 \text { Multi-objective Weight Determination for Set-based Portfolio }}$ Optimization

Let $t$ represent the current iteration;

Let $S_{k} \cdot X_{i}$ represent set-particle $i$ in MGSBPSO swarm $k$;

Minimize risk and maximize return using $\mathrm{MGPSO}_{\mathrm{s}}$ with assets in $X_{i}$ until no solutions are inserted into the $\mathrm{MGPSO}_{\mathrm{s}}$ archive;

$t=t+t_{w}$

Insert the non-dominated solutions in the $\mathrm{MGPSO}_{\mathrm{s}}$ archive with assets in $S_{k} \cdot X_{i}$ into the global archive;

if $k=1$ then

Return the best risk value, and corresponding weights, found by the $\mathrm{MGPSO}_{\mathrm{s}}$;

else

Return the best return value, and corresponding weights, found by the $\mathrm{MGPSO}_{\mathrm{s}}$; 


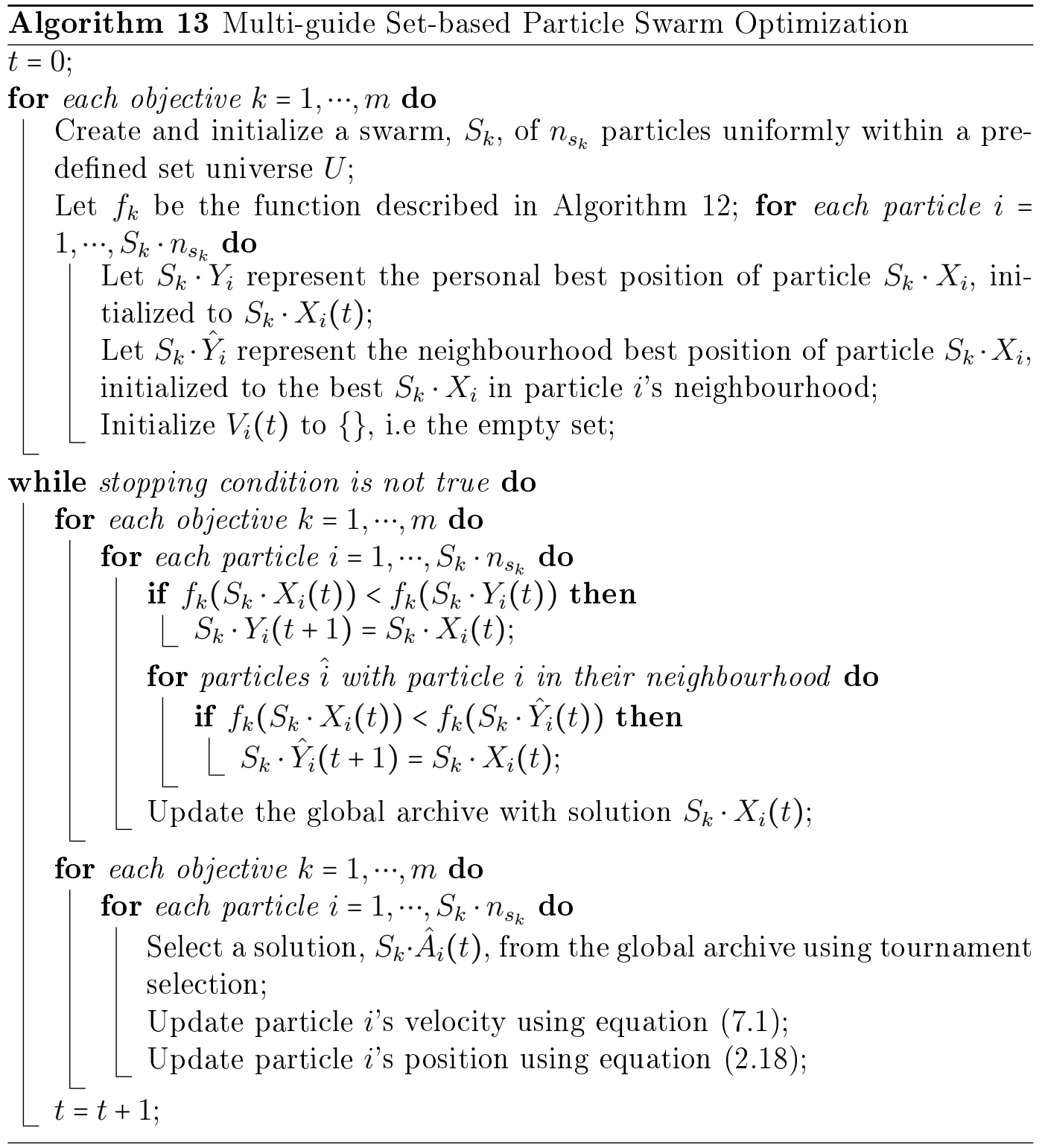

The proposed MGSBPSO is expected to perform similarly to the MGPSO for multi-objective portfolio optimization since MGSBPSO makes use of $\mathrm{MGPSO}_{\mathrm{s}}$ for asset weight optimization. It is also expected that the reduction in dimensionality by MGSBPSO will result in higher-quality solutions than $\mathrm{MGPSO}_{\mathrm{s}}$ for larger portfolio problems.

\subsection{Empirical Process}

The empirical process described in Chapter 4 is also used here to gauge the performance of MGSBPSO with the caveat that instead of using the meanvariance model of equation (3.1), risk (equation (3.2)) and return (equation 
(3.3)) are optimized independently. The performance of MGSBPSO is compared with that of $\mathrm{MGPSO}_{\mathrm{s}}$ as a standalone approach where solution representation is a fixed-length vector of floats. For each algorithm, a total swarm size of 50 is used with 25 particles allocated to each swarm. The archive size for each algorithm, as well as the global archive for MGSBPSO, is set to 50 . Furthermore, all swarms makes use of a gbest topology. The algorithms are allocated 5000 iterations per independent run. The performance of $\mathrm{SBPSO}_{\mathrm{m} 6}$ is also included in the performance assessment of MGSBPSO since it was shown to outperform other algorithms for single-objective portfolio optimization in the previous chapter. It should be noted that, for this experiment, the time performance measure refers to the time in seconds to obtain the POF, or in the case of $\mathrm{SBPSO}_{\mathrm{m} 6}$, the time in seconds to optimize equation (3.1) for 50 evenly spaced $\lambda$ values.

\subsection{Results}

This section discusses the results of MGPSO, MGSBPSO and $\mathrm{SBPSO}_{\mathrm{m} 6}$ for each benchmark problem. Section 7.4.1 examines the Hang Seng results. The DAX 100 and FTSE 100 results are discussed in Sections 7.4.2 and 7.4.3. respectively. Section 7.4 .4 discusses the S\&P 100 results, and the Nikkei 225 results are discussed in Section 7.4.5.

\subsubsection{Hang Seng}

Table 7.6 shows that $\mathrm{MGPSO}_{\mathrm{s}}$ and MGSBPSO performed similarly. Both algorithms obtained optimal solutions that were close to the true POF, as indicated by the GD and IGD scores, in a reasonable time. However, MGSBPSO was slightly more competitive than $\mathrm{MGPSO}_{\mathrm{s}}$ with regard to risk and return. MGSBPSO obtained portfolios that on average had a higher return value and lower risk value than $\mathrm{MGPSO}_{\mathrm{s}}$. $\mathrm{SBPSO}_{\mathrm{m} 6}$ by comparison obtained fewer portfolios that were much more profitable at a similar level of risk. Table 7.7 shows that the $\mathrm{SBPSO}_{\mathrm{m} 6}$ generally ranked first across the performance measures. However, $\mathrm{SBPSO}_{\mathrm{m} 6}$ ranked second overall as a result of ranking last in the number of optimal solutions obtained, $\mathrm{HV}$ and time to reach the stopping condition. MGPSO $_{\mathrm{s}}$ and MGSBPSO ranked first and second overall, respectively. MGSBPSO ranked first in time to reach the stopping condition. Figure 7.2 shows that MGSBPSO was able to approximate the true POF slightly better than $\mathrm{MGPSO}_{\mathrm{s}}$. 
CHAPTER 7. SET-BASED PARTICLE SWARM OPTIMIZATION FOR MULTI-OBJECTIVE PORTFOLIO OPTIMIZATION

Table 7.6: Hang Seng results for each performance measure

\begin{tabular}{lccccccccc}
\hline Algorithm & Result & $\mathbf{N}$ & $K$ & $R$ & $\bar{\sigma}$ & GD & IGD & HV & Time \\
\hline MGPSO $_{\mathrm{S}}$ & $\bar{x}$ & 43 & 14 & 0.007261 & 0.001967 & 0.000723 & 0.000218 & 1.192743 & 114.67 \\
& $\sigma$ & 0 & 2 & 0.000705 & 0.000407 & 0.000262 & 0.000025 & 0.002658 & 4.95 \\
\hline MGSBPSO & $\bar{x}$ & 47 & 5 & 0.007382 & 0.001814 & 0.000761 & 0.000252 & 1.190891 & 100.50 \\
& $\sigma$ & 0 & 1 & 0.000552 & 0.000326 & 0.000077 & 0.000029 & 0.001930 & 2.69 \\
\hline SBPSO $_{\mathrm{m} 6}$ & $\bar{x}$ & 33 & 3 & 0.007607 & 0.001897 & 0.000218 & 0.000212 & 0.781949 & 226.05 \\
& $\sigma$ & 0 & 0 & 0.000003 & 0.000000 & 0.000045 & 0.000002 & 0.026784 & 143.66 \\
\hline
\end{tabular}

Table 7.7: Hang Seng rankings for each performance measure

\begin{tabular}{llccccccccc}
\hline Algorithm & Result & N & $K$ & $R$ & $\bar{\sigma}$ & GD & IGD & HV & Time & Overall \\
\hline MGPSO $_{\text {s }}$ & Wins & 1 & 0 & 0 & 0 & 1 & 1 & 2 & 1 & 6 \\
& Losses & 0 & 2 & 0 & 0 & 1 & 0 & 0 & 1 & 4 \\
& Draws & 1 & 0 & 2 & 2 & 0 & 1 & 0 & 0 & 6 \\
& Difference & 1 & -2 & 0 & 0 & 0 & 1 & 2 & 0 & 2 \\
& Rank & 1 & 3 & 2 & 1 & 2 & 1 & 1 & 2 & 1 \\
\hline \multirow{2}{*}{ MGSBPSO } & Wins & 1 & 1 & 0 & 0 & 0 & 0 & 1 & 2 & 5 \\
& Losses & 0 & 1 & 1 & 0 & 2 & 2 & 1 & 0 & 7 \\
& Draws & 1 & 0 & 1 & 2 & 0 & 0 & 0 & 0 & 4 \\
& Difference & 1 & 0 & -1 & 0 & -2 & -2 & 0 & 2 & -2 \\
& Rank & 1 & 2 & 3 & 1 & 3 & 2 & 2 & 1 & 3 \\
\hline \multirow{2}{*}{ RPSO $_{\mathrm{m} 6}$} & Wins & 0 & 2 & 1 & 0 & 2 & 1 & 0 & 0 & 6 \\
& Losses & 2 & 0 & 0 & 0 & 0 & 0 & 2 & 2 & 6 \\
& Draws & 0 & 0 & 1 & 2 & 0 & 1 & 0 & 0 & 4 \\
& Difference & -2 & 2 & 1 & 0 & 2 & 1 & -2 & -2 & 0 \\
& Rank & 2 & 1 & 1 & 1 & 1 & 1 & 3 & 3 & 2 \\
\hline
\end{tabular}




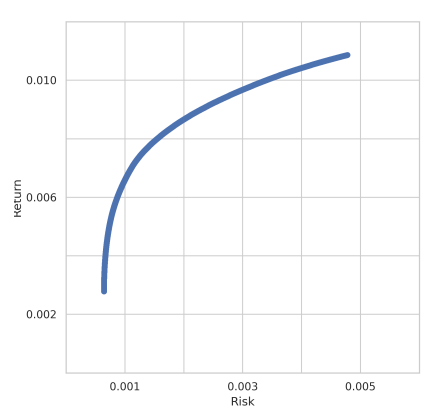

(a) True POF

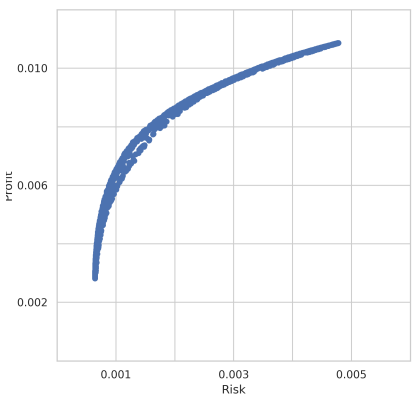

(b) $\mathrm{MGPSO}_{\mathrm{s}}$

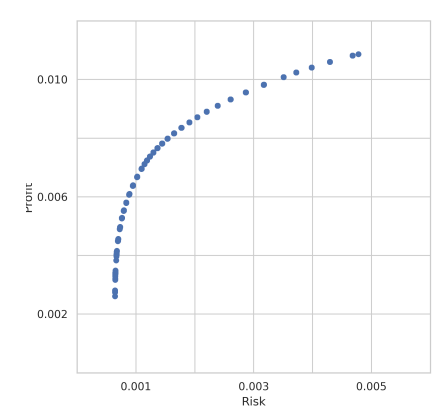

(d) $\mathrm{SBPSO}_{\mathrm{m} 6}$

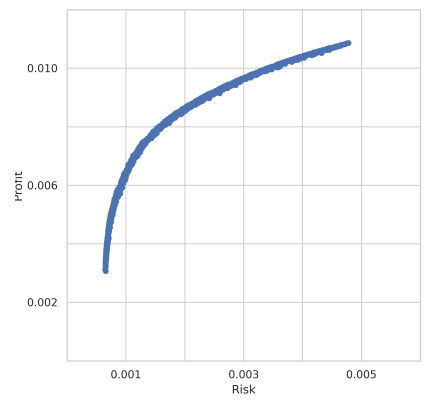

(c) MGSBPSO

Figure 7.2: Obtained Pareto-optimal fronts for Hang Seng

\subsubsection{DAX 100}

Table 7.8 shows that the MGSBPSO did not perform as well as $\mathrm{MGPSO}_{\mathrm{s}}$ and $\mathrm{SBPSO}_{\mathrm{m} 6}$ for the DAX 100 benchmark problem. Specifically, the MGSBPSO had the lowest average return value and an average risk value that was an order of magnitude worse than the risk values of the other two algorithms. Furthermore, MGSBPSO had the worst average GD value. However, the average values and the rankings in Table 7.9 show that the MGSBPSO performed well for IGD, HV and time to reach the stopping condition. As for the previous benchmark problem, $\mathrm{SBPSO}_{\mathrm{m} 6}$ took statistically significantly longer to find fewer optimal portfolios. $\mathrm{SBPSO}_{\mathrm{m} 6}$ also obtained a much lower $\mathrm{HV}$. The $M_{G P S O}$ ranked second for GD and first for IGD, HV, risk and the number of optimal solutions obtained. As a result, the MGPSO $\mathrm{S}_{\mathrm{s}}$ ranked first overall. However, $\mathrm{MGPSO}_{\mathrm{s}}$ obtained significantly larger portfolios - an order of magnitude larger than the portfolios of the other two algorithms. The obtained POFs in Figure 7.3 show that the MGSBPSO was able to approximate the true POF better than the $\mathrm{MGPSO}_{\mathrm{s}}$. The POF obtained by $\mathrm{SBPSO}_{\mathrm{m} 6}$ resembles the true POF the best. 
CHAPTER 7. SET-BASED PARTICLE SWARM OPTIMIZATION FOR MULTI-OBJECTIVE PORTFOLIO OPTIMIZATION

Table 7.8: DAX 100 results for each performance measure

\begin{tabular}{lccccccccc}
\hline Algorithm & Result & $\mathbf{N}$ & $K$ & $R$ & $\bar{\sigma}$ & GD & IGD & HV & Time \\
\hline MGPSO $_{\mathrm{S}}$ & $\bar{x}$ & 46 & 41 & 0.005960 & 0.000688 & 0.001058 & 0.000264 & 1.195303 & 266.54 \\
& $\sigma$ & 1 & 4 & 0.000459 & 0.000267 & 0.000490 & 0.000053 & 0.003118 & 5.26 \\
\hline MGSBPSO & $\bar{x}$ & 45 & 13 & 0.005838 & 0.005388 & 0.003119 & 0.000259 & 1.192224 & 203.25 \\
& $\sigma$ & 2 & 1 & 0.000388 & 0.026832 & 0.011012 & 0.000042 & 0.003413 & 9.77 \\
\hline SBPSO $_{\mathrm{m} 6}$ & $\bar{x}$ & 43 & 4 & 0.008469 & 0.000972 & 0.000250 & 0.000306 & 0.878138 & 449.54 \\
& $\sigma$ & 0 & 0 & 0.000006 & 0.000000 & 0.000061 & 0.000008 & 0.065429 & 159.43 \\
\hline
\end{tabular}

Table 7.9: DAX 100 rankings for each performance measure

\begin{tabular}{llccccccccc}
\hline Algorithm & Result & N & $K$ & $R$ & $\bar{\sigma}$ & GD & IGD & HV & Time & Overall \\
\hline MGPSO $_{\text {S }}$ & Wins & 1 & 0 & 0 & 2 & 1 & 1 & 2 & 1 & 8 \\
& Losses & 0 & 2 & 1 & 0 & 1 & 0 & 0 & 1 & 5 \\
& Draws & 1 & 0 & 1 & 0 & 0 & 1 & 0 & 0 & 3 \\
& Difference & 1 & -2 & -1 & 2 & 0 & 1 & 2 & 0 & 3 \\
& Rank & 1 & 3 & 2 & 1 & 2 & 1 & 1 & 2 & 1 \\
\hline \multirow{2}{*}{ MGSBPSO $^{*}$ Wins } & 1 & 1 & 0 & 0 & 0 & 1 & 1 & 2 & 6 \\
& Losses & 0 & 1 & 1 & 2 & 2 & 0 & 1 & 0 & 7 \\
& Draws & 1 & 0 & 1 & 0 & 0 & 1 & 0 & 0 & 3 \\
& Difference & 1 & 0 & -1 & -2 & -2 & 1 & 0 & 2 & -1 \\
& Rank & 1 & 2 & 2 & 3 & 3 & 1 & 2 & 1 & 2 \\
\hline \multirow{2}{*}{ LPSO $_{\mathrm{m} 6}$} & Wins & 0 & 2 & 2 & 1 & 2 & 0 & 0 & 0 & 7 \\
& Losses & 2 & 0 & 0 & 1 & 0 & 2 & 2 & 2 & 9 \\
& Draws & 0 & 0 & 0 & 0 & 0 & 0 & 0 & 0 & 0 \\
& Difference & -2 & 2 & 2 & 0 & 2 & -2 & -2 & -2 & -2 \\
& Rank & 2 & 1 & 1 & 2 & 1 & 2 & 3 & 3 & 3 \\
\hline
\end{tabular}




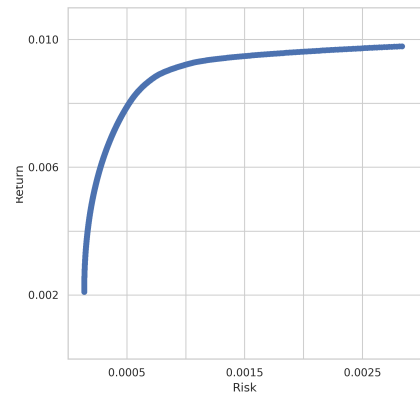

(a) True POF

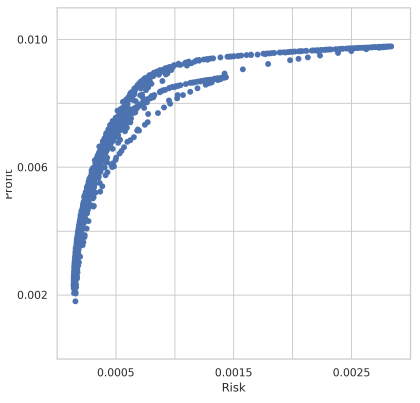

(b) $\mathrm{MGPSO}_{\mathrm{s}}$

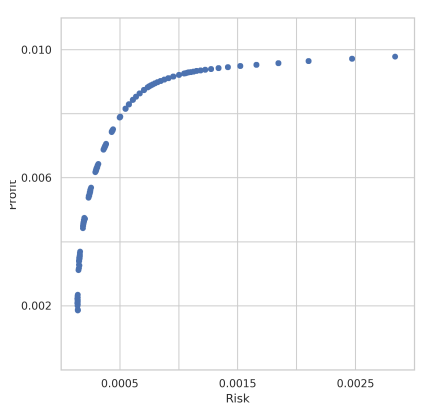

(d) $\mathrm{SBPSO}_{\mathrm{m} 6}$

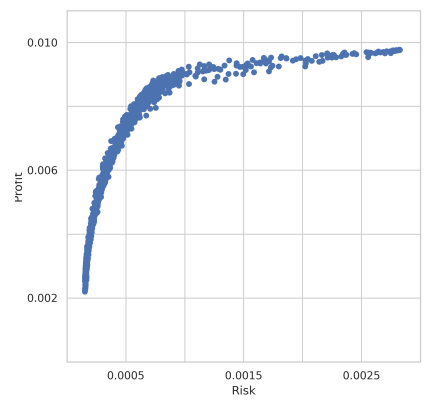

(c) MGSBPSO

Figure 7.3: Obtained Pareto-optimal fronts for DAX 100

\subsubsection{FTSE 100}

Table 7.14 shows that the MGSBPSO performed better than the MGPSO for the size of the obtained portfolios, risk, GD, and IGD. Although MGSBPSO did take less time on average than the $\mathrm{MGPSO}_{\mathrm{s}}$ to reach the stopping condition, the standard deviation of the time taken by MGSBPSO was much larger than that of MGPSO $_{\text {s. }}$. Table 7.15 shows that overall the MGSBPSO ranked first, $\mathrm{SBPSO}_{\mathrm{m} 6}$ ranked second and $\mathrm{MGPSO}_{\mathrm{s}}$ ranked third. The $\mathrm{SBPSO}_{\mathrm{m} 6}$ obtained smaller sized portfolios that were more profitable than those obtained by the other two algorithms, but with higher risk. However, the $\mathrm{SBPSO}_{\mathrm{m} 6}$ was much slower to reach the stopping condition than $\mathrm{MGPSO}_{\mathrm{s}}$ and MGSBPSO. A visual analysis of the obtained POFs in Figure 7.6 shows that $\mathrm{MGPSO}_{\mathrm{s}}$ and MGSBPSO were somewhat able to approximate the true POF. The POFs of both algorithms contained non-dominated points that were present in the true POF, but also points that were dominated by the true POF. 
CHAPTER 7. SET-BASED PARTICLE SWARM OPTIMIZATION FOR MULTI-OBJECTIVE PORTFOLIO OPTIMIZATION

Table 7.10: FTSE 100 results for each performance measure

\begin{tabular}{lccccccccc}
\hline Algorithm & Result & $\mathbf{N}$ & $K$ & $R$ & $\bar{\sigma}$ & GD & IGD & HV & Time \\
\hline MGPSO $_{\mathrm{S}}$ & $\bar{x}$ & 44 & 47 & 0.005239 & 0.000570 & 0.001111 & 0.000258 & 1.189333 & 264.95 \\
& $\sigma$ & 2 & 8 & 0.000508 & 0.000097 & 0.000606 & 0.000127 & 0.003496 & 4.08 \\
\hline MGSBPSO & $\bar{x}$ & 47 & 14 & 0.005079 & 0.000438 & 0.001003 & 0.000241 & 1.186224 & 233.95 \\
& $\sigma$ & 0 & 1 & 0.000249 & 0.000048 & 0.000076 & 0.000026 & 0.001914 & 68.16 \\
\hline SBPSO $_{\mathrm{m} 6}$ & $\bar{x}$ & 30 & 5 & 0.006683 & 0.000785 & 0.000247 & 0.000235 & 0.741994 & 528.62 \\
& $\sigma$ & 0 & 0 & 0.000007 & 0.000001 & 0.000038 & 0.000007 & 0.049170 & 177.55 \\
\hline
\end{tabular}

Table 7.11: FTSE 100 rankings for each performance measure

\begin{tabular}{|c|c|c|c|c|c|c|c|c|c|c|}
\hline Algorithm & Result & $\mathbf{N}$ & $K$ & $R$ & $\bar{\sigma}$ & GD & IGD & HV & Time & Overall \\
\hline \multirow{5}{*}{$\mathrm{MGPSO}_{\mathrm{s}}$} & Wins & 1 & 0 & 1 & 1 & 0 & 0 & 2 & 1 & 6 \\
\hline & Losses & 1 & 2 & 1 & 1 & 2 & 2 & 0 & 1 & 10 \\
\hline & Draws & 0 & 0 & 0 & 0 & 0 & 0 & 0 & 0 & 0 \\
\hline & Difference & 0 & -2 & 0 & 0 & -2 & -2 & 2 & 0 & -4 \\
\hline & Rank & 2 & 3 & 2 & 2 & 3 & 2 & 1 & 2 & 3 \\
\hline \multirow[t]{5}{*}{ MGSBPSO } & Wins & 2 & 1 & 0 & 2 & 1 & 1 & 1 & 2 & 10 \\
\hline & Losses & 0 & 1 & 2 & 0 & 1 & 0 & 1 & 0 & 5 \\
\hline & Draws & 0 & 0 & 0 & 0 & 0 & 1 & 0 & 0 & 1 \\
\hline & Difference & 2 & 0 & -2 & 2 & 0 & 1 & 0 & 2 & 5 \\
\hline & Rank & 1 & 2 & 3 & 1 & 2 & 1 & 2 & 1 & 1 \\
\hline \multirow[t]{5}{*}{$\mathrm{SBPSO}_{\mathrm{m} 6}$} & Wins & 0 & 2 & 2 & 0 & 2 & 1 & 0 & 0 & 7 \\
\hline & Losses & 2 & 0 & 0 & 2 & 0 & 0 & 2 & 2 & 8 \\
\hline & Draws & 0 & 0 & 0 & 0 & 0 & 1 & 0 & 0 & 1 \\
\hline & Difference & -2 & 2 & 2 & -2 & 2 & 1 & -2 & -2 & -1 \\
\hline & Rank & 3 & 1 & 1 & 3 & 1 & 1 & 3 & 3 & 2 \\
\hline
\end{tabular}




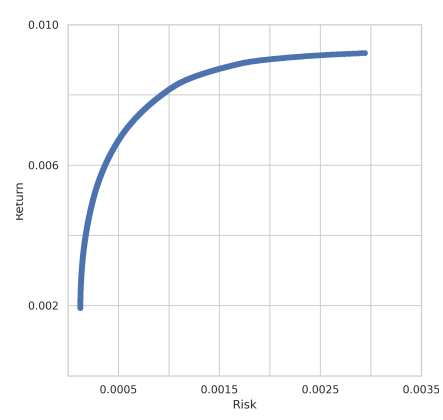

(a) True POF

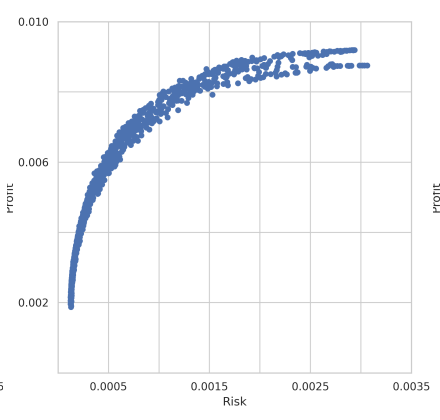

(b) $\mathrm{MGPSO}_{\mathrm{s}}$

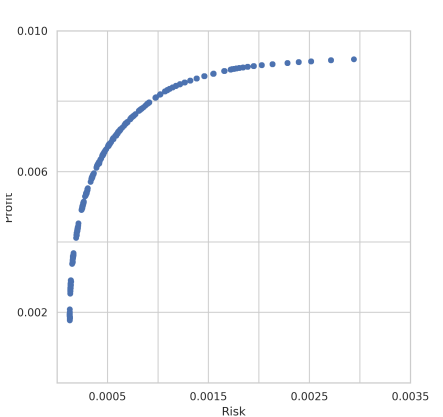

(d) $\mathrm{SBPSO}_{\mathrm{m} 6}$

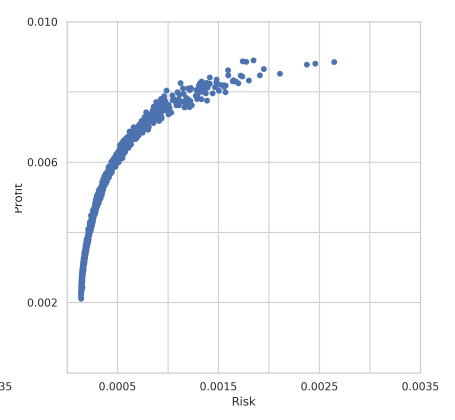

(c) MGSBPSO

Figure 7.4: Obtained Pareto-optimal fronts for FTSE 100

\subsubsection{S\&P 100}

The average results in Table 7.12 shows that the MGSBPSO performed well for this benchmark problem. Specifically, the MGSBPSO performed better than $\mathrm{MGPSO}_{\mathrm{s}}$ for the GD measure and better than $\mathrm{SBPSO}_{\mathrm{m} 6}$ for the HV measure. Furthermore, MGSBPSO obtained portfolios with the least amount of risk and it was the fastest algorithm to reach the stopping condition. The $\mathrm{MGPSO}_{\mathrm{s}}$ also performed well, particularly for $\mathrm{HV}$, but it obtained much larger portfolios than MGSBPSO and $\mathrm{SBPSO}_{\mathrm{m} 6}$. The rankings in Table 7.13 show that the MGSBPSO was overall the best performing algorithm. The MGPSO ranked last overall, but ranked higher than MGSBPSO for the Pareto optimality measures. Furthermore, $\mathrm{MGPSO}_{\mathrm{s}}$ ranked higher than $\mathrm{SBPSO}_{\mathrm{m} 6}$ for the $\mathrm{HV}$ measure. However, Figure 7.5 shows that the $\mathrm{MGPSO}_{\mathrm{s}}$ did not approximate the true POF as well as $\mathrm{SBPSO}_{\mathrm{m} 6}$ or MGSBPSO. Furthermore, the POFs obtained by $\mathrm{MGPSO}_{\mathrm{s}}$ and MGSBPSO contains points that are dominated by the true POF. 
CHAPTER 7. SET-BASED PARTICLE SWARM OPTIMIZATION FOR MULTI-OBJECTIVE PORTFOLIO OPTIMIZATION

Table 7.12: S\&P 100 results for each performance measure

\begin{tabular}{lccccccccc}
\hline Algorithm & Result & $\mathbf{N}$ & $K$ & $R$ & $\bar{\sigma}$ & GD & IGD & HV & Time \\
\hline MGPSO $_{\mathrm{S}}$ & $\bar{x}$ & 48 & 65 & 0.004993 & 0.000579 & 0.001344 & 0.000276 & 1.195282 & 281.01 \\
& $\sigma$ & 0 & 4 & 0.000359 & 0.000150 & 0.000235 & 0.000037 & 0.001887 & 4.30 \\
\hline MGSBPSO & $\bar{x}$ & 48 & 21 & 0.004819 & 0.000388 & 0.001263 & 0.000330 & 1.190151 & 220.53 \\
& $\sigma$ & 0 & 2 & 0.000195 & 0.000053 & 0.000099 & 0.000038 & 0.001808 & 9.91 \\
\hline SBPSO $_{\mathrm{m} 6}$ & $\bar{x}$ & 44 & 6 & 0.007716 & 0.001248 & 0.000275 & 0.000247 & 0.875057 & 822.26 \\
& $\sigma$ & 0 & 0 & 0.000006 & 0.000001 & 0.000044 & 0.000003 & 0.044175 & 139.06 \\
\hline
\end{tabular}

Table 7.13: S\&P 100 rankings for each performance measure

\begin{tabular}{|c|c|c|c|c|c|c|c|c|c|c|}
\hline Algorithm & Result & $\mathbf{N}$ & $K$ & $R$ & $\bar{\sigma}$ & GD & IGD & HV & Time & Overall \\
\hline \multirow{5}{*}{$\mathrm{MGPSO}_{\mathrm{s}}$} & Wins & 1 & 0 & 1 & 1 & 0 & 1 & 2 & 1 & 7 \\
\hline & Losses & 1 & 2 & 1 & 1 & 1 & 1 & 0 & 1 & 8 \\
\hline & Draws & 0 & 0 & 0 & 0 & 1 & 0 & 0 & 0 & 1 \\
\hline & Difference & 0 & -2 & 0 & 0 & -1 & 0 & 2 & 0 & -1 \\
\hline & Rank & 2 & 3 & 2 & 2 & 2 & 2 & 1 & 2 & 3 \\
\hline \multirow[t]{5}{*}{ MGSBPSO } & Wins & 2 & 1 & 0 & 2 & 0 & 0 & 1 & 2 & 8 \\
\hline & Losses & 0 & 1 & 2 & 0 & 1 & 2 & 1 & 0 & 7 \\
\hline & Draws & 0 & 0 & 0 & 0 & 1 & 0 & 0 & 0 & 1 \\
\hline & Difference & 2 & 0 & -2 & 2 & -1 & -2 & 0 & 2 & 1 \\
\hline & Rank & 1 & 2 & 3 & 1 & 2 & 3 & 2 & 1 & 1 \\
\hline \multirow[t]{5}{*}{$\mathrm{SBPSO}_{\mathrm{m} 6}$} & Wins & 0 & 2 & 2 & 0 & 2 & 2 & 0 & 0 & 8 \\
\hline & Losses & 2 & 0 & 0 & 2 & 0 & 0 & 2 & 2 & 8 \\
\hline & Draws & 0 & 0 & 0 & 0 & 0 & 0 & 0 & 0 & 0 \\
\hline & Difference & -2 & 2 & 2 & -2 & 2 & 2 & -2 & -2 & 0 \\
\hline & Rank & 3 & 1 & 1 & 3 & 1 & 1 & 3 & 3 & 2 \\
\hline
\end{tabular}




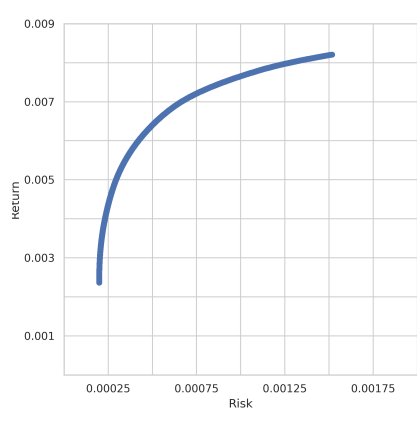

(a) True POF

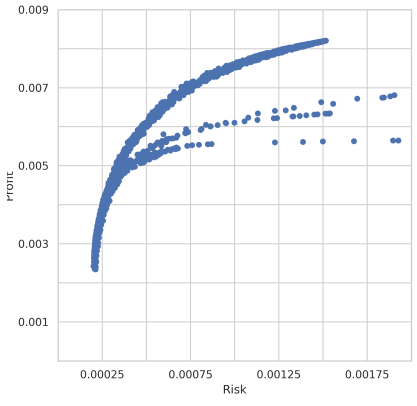

(b) $\mathrm{MGPSO}_{\mathrm{s}}$

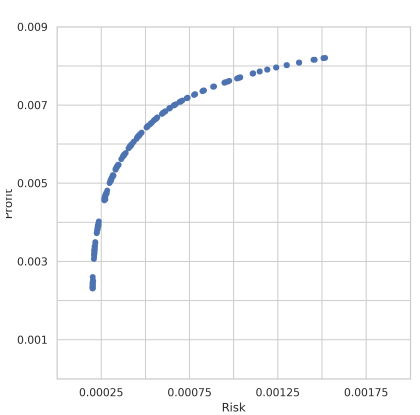

(d) $\mathrm{SBPSO}_{\mathrm{m} 6}$

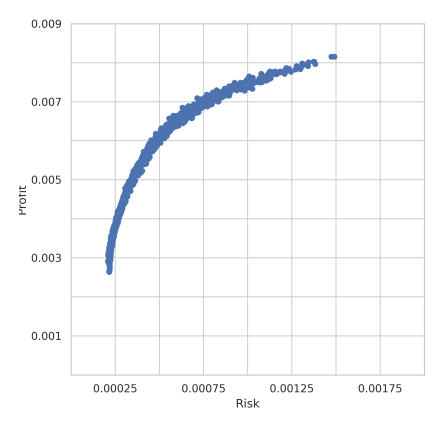

(c) MGSBPSO

Figure 7.5: Obtained Pareto-optimal fronts for S\&P 100

\subsubsection{Nikkei 225}

The MGSBPSO was more competitive than $\mathrm{MGPSO}_{\mathrm{s}}$ with regard to risk and return for this benchmark problem, as shown in Table 7.14. MGSBPSO obtained more profitable and less risky portfolios than $\mathrm{MGPSO}_{\mathrm{s}}$ while being faster to reach the stopping condition. Furthermore, the MGSBPSO obtained more optimal portfolios than $\mathrm{MGPSO}_{\mathrm{s}}$ and portfolios with fewer assets than $M_{G P S O}$. Table 7.15 shows that MGSBPSO ranked higher than MGPSO with regard to GD and IGD, and ranked first overall. $\mathrm{SBPSO}_{\mathrm{m} 6}$ was approximately three times slower than the $\mathrm{MGPSO}_{\mathrm{s}}$ to reach the stopping condition and four and a half times slower than MGSBPSO. The $\mathrm{SBPSO}_{\mathrm{m} 6}$ ranked second overall and the $\mathrm{MGPSO}_{\mathrm{s}}$ ranked last. Figure 7.6 shows that the $\mathrm{MGPSO}_{\mathrm{s}}$ was unable to approximate the true POF, unlike MGSBPSO and $\mathrm{SBPSO}_{\mathrm{m} 6}$. 
CHAPTER 7. SET-BASED PARTICLE SWARM OPTIMIZATION FOR MULTI-OBJECTIVE PORTFOLIO OPTIMIZATION

Table 7.14: Nikkei 225 results for each performance measure

\begin{tabular}{lccccccccc}
\hline Algorithm & Result & $\mathbf{N}$ & $K$ & $R$ & $\bar{\sigma}$ & GD & IGD & HV & Time \\
\hline MGPSO $_{\mathrm{S}}$ & $\bar{x}$ & 45 & 22 & 0.002193 & 0.000650 & 0.001631 & 0.000294 & 1.190877 & 996.02 \\
& $\sigma$ & 0 & 5 & 0.000338 & 0.000122 & 0.000393 & 0.000051 & 0.003675 & 11.07 \\
\hline MGSBPSO & $\bar{x}$ & 49 & 5 & 0.002437 & 0.000633 & 0.001242 & 0.000242 & 1.191968 & 679.75 \\
& $\sigma$ & 0 & 1 & 0.000272 & 0.000113 & 0.000114 & 0.000030 & 0.003169 & 69.26 \\
\hline SBPSO $_{\mathrm{m} 6}$ & $\bar{x}$ & 44 & 4 & 0.003310 & 0.000794 & 0.000260 & 0.000223 & 0.916014 & 3159.48 \\
& $\sigma$ & 0 & 0 & 0.000007 & 0.000001 & 0.000060 & 0.000005 & 0.047097 & 306.54 \\
\hline
\end{tabular}

Table 7.15: Nikkei 225 rankings for each performance measure

\begin{tabular}{|c|c|c|c|c|c|c|c|c|c|c|}
\hline Algorithm & Result & $\mathbf{N}$ & $K$ & $R$ & $\bar{\sigma}$ & GD & IGD & HV & Time & Overall \\
\hline \multirow{5}{*}{$\mathrm{MGPSO}_{\mathrm{s}}$} & Wins & 1 & 0 & 0 & 1 & 0 & 0 & 1 & 1 & 4 \\
\hline & Losses & 1 & 2 & 2 & 0 & 2 & 2 & 0 & 1 & 10 \\
\hline & Draws & 0 & 0 & 0 & 1 & 0 & 0 & 1 & 0 & 2 \\
\hline & Difference & 0 & -2 & -2 & 1 & -2 & -2 & 1 & 0 & -6 \\
\hline & Rank & 2 & 3 & 3 & 1 & 3 & 3 & 1 & 2 & 3 \\
\hline \multirow[t]{5}{*}{ MGSBPSO } & Wins & 2 & 1 & 1 & 1 & 1 & 1 & 1 & 2 & 10 \\
\hline & Losses & 0 & 1 & 1 & 0 & 1 & 1 & 0 & 0 & 4 \\
\hline & Draws & 0 & 0 & 0 & 1 & 0 & 0 & 1 & 0 & 2 \\
\hline & Difference & 2 & 0 & 0 & 1 & 0 & 0 & 1 & 2 & 6 \\
\hline & Rank & 1 & 2 & 2 & 1 & 2 & 2 & 1 & 1 & 1 \\
\hline \multirow[t]{5}{*}{$\mathrm{SBPSO}_{\mathrm{m} 6}$} & Wins & 0 & 2 & 2 & 0 & 2 & 2 & 0 & 0 & 8 \\
\hline & Losses & 2 & 0 & 0 & 2 & 0 & 0 & 2 & 2 & 8 \\
\hline & Draws & 0 & 0 & 0 & 0 & 0 & 0 & 0 & 0 & 0 \\
\hline & Difference & -2 & 2 & 2 & -2 & 2 & 2 & -2 & -2 & 0 \\
\hline & Rank & 3 & 1 & 1 & 2 & 1 & 1 & 2 & 3 & 2 \\
\hline
\end{tabular}




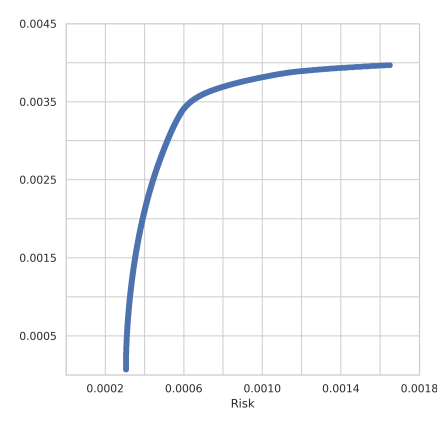

(a) True POF

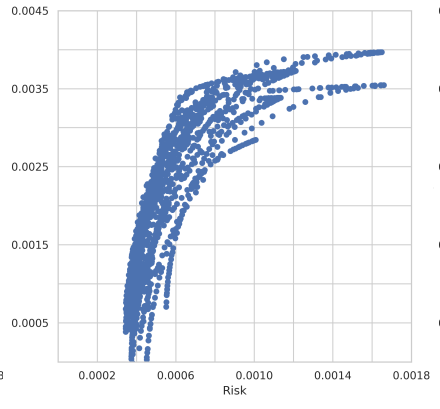

(b) $\mathrm{MGPSO}_{\mathrm{s}}$

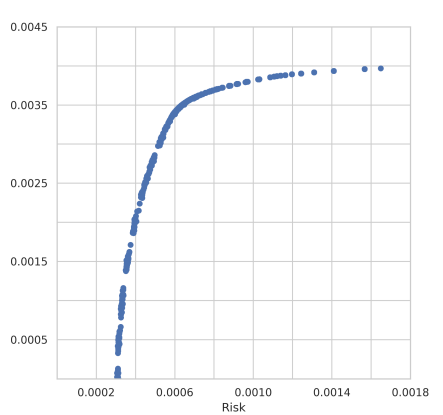

(d) $\mathrm{SBPSO}_{\mathrm{m} 6}$

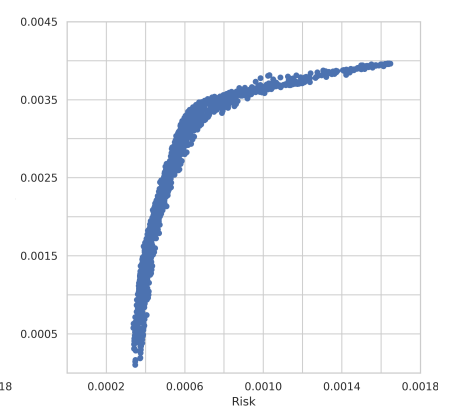

(c) MGSBPSO

Figure 7.6: Obtained Pareto-optimal fronts for Nikkei 225

\subsection{Summary}

This chapter proposed the multi-guide set-based particle swarm optimization (MGSBPSO) algorithm, a multi-objective adaptation of the set-based particle swarm optimization (SBPSO) algorithm that incorporates elements from the multi-guide particle swarm optimization (MGPSO) algorithm. For the purpose of optimising the asset weights, MGPSO was used. To guarantee that MGPSO finds high-quality solutions, its control parameters have to be tuned. Since parameter tuning is an expensive process, an alternative method to randomly sample control parameters values from the stability region defined in equation (2.8) was investigated. It was found that the accuracy of MGPSO using this approach, i.e. $\mathrm{MGPSO}_{\mathrm{s}}$, was similar to that of MGPSO using tuned control parameter values. Thus, $\mathrm{MGPSO}_{\mathrm{s}}$ was used as the weight optimizer for MGSBPSO, as well as a standalone algorithm to compare the results of MGSBPSO with. Furthermore, SBPSO with the modifications presented in Chapter 6 was also used to assess the performance of MGSBPSO. The results showed that both the MGSBPSO and MGPSO $\mathrm{s}$ performed well for multi-objective portfolio optimization. However, the $\mathrm{MGPSO}_{\mathrm{s}}$ did not scale to larger portfolio problems as well as MGSBPSO and the SBPSO. The search time of MGSBPSO was faster than that of $\mathrm{MGPSO}_{\mathrm{s}}$ and the SBPSO with modifications for all benchmark problems. Furthermore, the ability of 
CHAPTER 7. SET-BASED PARTICLE SWARM OPTIMIZATION FOR MULTI-OBJECTIVE PORTFOLIO OPTIMIZATION

$\mathrm{MGPSO}_{\mathrm{s}}$ to approximate the true POF deteriorated as the size of the portfolio problems increased whereas the performance of MGSBPSO was somewhat consistent across benchmark problems. The SBPSO with modifications also performed well. However, it was optimising the mean-variance portfolio model of equation (3.1) for a given risk-return tradeoff value, which does require that risk and return are optimised independently - which is what MGSBPSO and MGPSO $_{\mathrm{s}}$ did. Furthermore, the SBPSO with modifications only found a single solution after each independent run, whereas MGSBPSO and $\mathrm{MGPSO}_{\mathrm{s}}$ found many non-dominated solutions. Overall, the results show that the benefits of redefining the portfolio optimization problem as a set-based problem are also applicable to multi-objective portfolio optimization. 


\section{Chapter 8}

\section{Conclusions}

This study proposed a set-based particle swarm optimization (SBPSO) for the portfolio optimization problem, set-based diversity measures, improvements to the SBPSO for portfolio optimization, a version of multi-guide particle swarm optimization (MGPSO) that does not require parameter tuning, and the first multi-objective SBPSO. The purpose of this chapter is to summarise the major findings of this study and to provide avenues for future research.

Section 8.1 presents a summary of the major findings and conclusions of this thesis, followed by suggestions for future work in Section 8.2 .

\subsection{Major Findings and Conclusions}

A literature review of over 100 publications from 1993 to 2019 found that a variety of evolutionary and swarm intelligence algorithms have been applied to portfolio optimization. The most popular of which are particle swarm optimization (PSO) and genetic algorithms (GAs). Lesser known algorithms, such as cat swarm optimization (CSO), chemical reaction optimization (CRO), firefly algorithm (FA), fireworks algorithm (FWA), krill Herd (KH) and invasive weed optimization (IWO) have also been studied, albeit to a lesser degree than PSO and GAs. In fact, many of these lesser known algorithms have only been applied to portfolio optimization once or twice. Researchers should ask themselves if the work being proposed is truly novel or to simply cash in on a trend of deriving meta-heuristics from nature.

The review also found that the majority of the work considered portfolio optimization as a single-objective optimization problem. This is done by introducing a trade-off coefficient to balance the risk and profit objectives. Thus, by optimizing for varying values of the trade-off coefficient, a set of optimal solutions can be obtained. However, once constraints are introduced, these approaches are effectively blind to solutions that cannot be obtained by optimizing for a given trade-off weighting. Multi-objective approaches have been shown to be capable of obtaining higher quality solutions while under 
constraints.

Since its inception in 2000, the cardinality constrained portfolio problem (CCPP) was the most widely considered constrained portfolio optimization problem. It places a limit on the number of assets included in the portfolio and bounds on how much an asset can contribute to the overall portfolio. The data proposed alongside the CCPP has also become extremely popular. A variety of other constrained portfolio problems and data sets have been studied, although they have not gained as much traction. The success of CCPP is largely due to it being well researched and clearly defined. Furthermore, the corresponding data set is publicly available online - the unconstrained version is used in this study.

No work has yet considered evolutionary and swarm intelligence algorithms for dynamic portfolio optimization. Dynamic portfolio optimization mimics high-frequency trading, such as daily trading. However, the lack of interest in this field is possibly due to the complexity it introduces.

The last key finding of the literature was that existing approaches suffer from stability issues when the size of the portfolio optimization problem increases, providing the motivation for this study: to propose a new scalable approach to portfolio optimization using SBPSO. The proposed approach makes use of a bi-stage search process that first selects assets and then determines the weights for said assets. They key idea behind this search process is that by selectively choosing which assets to optimize, the complexity of the problem reduces through a reduction in dimensionality. Set-based approaches have been used before, although with quadratic programming. The proposed approach uses a traditional PSO for weight optimization. An empirical analysis showed that SBPSO was capable of identifying good-quality solutions while being relatively fast. It performed better than the firefly algorithm (FA), but worse than artificial bee colony (ABC), a GA and PSO. Nevertheless, it was concluded that redefining the portfolio optimization problem as a set-based asset selection problem is an effective method for reducing the duration of the search, while still obtaining acceptable levels of performance.

Set-based diversity measures were proposed in order to investigate the performance and convergence behaviour of SBPSO, namely Jaccard diversity and Hamming diversity based on their respective distance measures. Both measures were able to represent the diversity of sets over time. It was found that Jaccard diversity better represents a researcher's intuition about the diversity of sets.

Using the newly defined Jaccard diversity, the effects of several SBPSO modifications were studied. In particular, the introduction of a linearly increasing control parameter that balanced exploratory and exploitative behaviour significantly improved the ability of SBPSO to converge to a single solution. Likewise, allowing the weight optimizer to execute until there is no improvement in solution quality, instead of a fixed number of time steps, resulted in large gains in performance. Adaptive coordinate descent (ACD) was used as 
a completely deterministic approach to weight determination. Performance analysis showed that SBPSO using ACD found higher-quality portfolios, but at the cost of a longer search time. Hence, PSO is still the preferred weight determination approach. The performance of SBPSO with the modifications and the ACD variant was evaluated against the aforementioned ABC, FA, GA and PSO as well as the original SBPSO for portfolio optimization. It was found that SBPSO with modifications and the ACD variant were statistically significantly better than the other algorithms in many ways.

A method to randomly sample control parameter values for MGPSO that satisfy stability conditions was investigated, with the motivation that it would allow researchers to easily use MGPSO to find optimal solutions to other problems without the need for parameter tuning. It was shown through empirical analysis that randomly sampling control parameter values that satisfy stability conditions yields similar performance to that of MGPSO using tuned parameters, and therefore is a viable alternative to parameter tuning.

A multi-objective adaptation of SBPSO was proposed, namely multi-guide set-based particle swarm optimization (MGSBPSO). MGSBPSO is a combination of SBPSO with the multi-objective components of MGPSO. MGSBPSO also makes use of the successful modifications mentioned earlier, and uses the MGPSO with the approach of randomly sampling control parameter values from the stability region as the weight optimizer. It was shown that MGSBPSO scaled to larger portfolio optimization problems better than MGPSO.

\subsection{Future Work}

This study concludes with the following ideas for future research:

\section{Maintaining Weights}

SBPSO for portfolio optimization uses a weight determination process to calculate the objective function and to identify good-performing assets. However, the asset weights are forgotten between weight determination stages. Thus, future work should explore strategies for maintaining and sharing goodquality weights between weight optimization searches. A potential solution is to archive the asset weights, which are updated over time. A population-based weight optimizer can then make use of the archive when initialising its population to make it partly aware of previously found fruitful areas. Because of this, care needs to be taken to avoid the weight optimizer prematurely converging on a solution. 


\section{Alternative Weight Optimizers}

This study examined one alternative weight optimizer, ACD, which helped SBPSO to find low risk portfolios. There is potentially performance to be gained by investigating other alternative weight optimizers. For example, GAs and $\mathrm{ABC}$ which were shown to be well studied approaches to portfolio optimization in the Chapter 3. Likewise, these algorithms could also benefit from the reduction in dimensionality that SBPSO provides.

\section{Set-based Particle Swarm Optimization for Constrained Portfolio Optimization}

SBPSO could easily be adapted to handle several constraints. For example, cardinality constraints and asset class constraints can be handled by SBPSO, creating an unconstrained weight determination environment. The research done here could also be applied to other constrained set-based optimization problems.

\section{Set-based Particle Swarm Optimization for Dynamic Portfolio Optimization}

A study of dynamic portfolio optimization requires a review of existing approaches to dynamic combinatorial optimization problems, from which a new dynamic version of SBPSO can be developed. Likewise, current literature on dynamic evolutionary algorithms (EAs) and SI algorithms can be applied to dynamic portfolio optimization, which would be a first. The proposed algorithm can later be used as a dynamic weight optimizer. How the dynamic SBPSO and weight optimizer would interact with each other is not immediately obvious and should be investigated. For example, would the dimensionality of the weight determination search space change as assets are added or removed from corresponding set-particles? If so, how would that affect the current solutions of the weight optimizer? Also, if each set-particle spawns a weight determination process, and these are evaluated sequentially, how would that process be adapted to dynamic environments? Perhaps concurrently? These questions would need to be investigated as a part of this work.

\section{Multi-guide Set-based Particle Swarm Optimization for Multi-objective Combinatorial Optimization Problems}

MGSBPSO demonstrated good performance for multi-objective portfolio optimization. Future work should investigate the performance of MGSBPSO for traditional multi-objective combinatorial problems, such as multi-objective knapsack problems. A part of this investigation would be to study the diver- 
sity of sub-swarms over time, as well as the effects of various parameter sets for different problems.

\section{A Modern Portfolio Optimization Benchmark Library}

This area of future work should attempt to propose a successor to the extremely popular portfolio optimization benchmarks of the OR Library. The benchmarks are well-researched and can be used for unconstrained portfolio optimization and the cardinality constrained portfolio problem. However, these benchmarks were first proposed 20 years ago, and since then there have been many constraints and objectives put forward. A modern portfolio optimization library should provide the necessary data to be compatible with most portfolio models and allow for additional constraints. Furthermore, the data sets should include in-sample and out-of-sample data to evaluate performance of the obtained portfolios in the real world. The data selected should be indicative of real world scenarios, such as market crashes and booms. Ideally, stock market indices should also be included to see if the algorithms are able to beat the market. An opinionated constrained portfolio model that extends the CCPP should also be given. Hopefully, this will encourage users to investigate more difficult problems. Lastly, the data sets should be publicly accessible and easy to use.

\section{Prediction Models}

There has yet to be any work done on using evolutionary and swarm intelligence algorithms to predict the prices of assets and make investment decisions based on these predictions. Investigation of such an approach will require researching and reviewing prediction models which can be optimized by an algorithm. The knowledge gained from the prediction models can then be used by the same algorithm, or perhaps a different one, to optimize a portfolio model. This area of work potentially overlaps with the need for a modern portfolio optimization library due to the data sets required. The work done here can also be extended to dynamic environments. 


\section{Bibliography}

[1] J. E. Alvarez-Benitez, R. M. Everson, and J. E. Fieldsend, "A MOPSO algorithm based exclusively on Pareto dominance concepts", in Proceedings of the International Conference on Evolutionary Multi-Criterion Optimization, Springer, 2005, pp. 459-473.

[2] K. P. Anagnostopoulos and G. Mamanis, "A portfolio optimization model with three objectives and discrete variables", Computers 83 Operations Research, vol. 37, no. 7, pp. 1285-1297, 2010.

[3] _ _ "Multiobjective evolutionary algorithms for complex portfolio optimization problems", Computational Management Science, vol. 8, no. 3, pp. 259279, Nov. 2009, ISSN: 1619-6988.

[4] —_ "The mean variance cardinality constrained portfolio optimization problem: An experimental evaluation of five multi-objective evolutionary algorithms", Expert Systems with Applications, vol. 38, no. 11, 2011, ISSN: 09574174 .

[5] C. Aranha and H. Iba, "The memetic tree-based genetic algorithm and its application to portfolio optimization", Memetic Computing, vol. 1, no. 2, pp. 139-151, Jun. 2009, ISSN: 1865-9292.

[6] R. Armananzas and J. A. Lozano, "A multi-objective approach to the portfolio optimization problem", in Proceedings of the IEEE Congress on Evolutionary Computation, vol. 2, Sep. 2005, 1388-1395 Vol. 2.

[7] S. Arnone, A. Loraschi, and A. Tettamanzi, "A genetic approach to portfolio selection", Neural Network World, vol. 3, pp. 597-604, Jan. 1993.

[8] N. Bacanin and M. Tuba, "Firefly Algorithm for cardinality constrained meanvariance portfolio optimization problem with entropy diversity constraint", The Scientific World Journal, vol. 2014, 2014.

[9] __ "Fireworks algorithm applied to constrained portfolio optimization problem", in Proceedings of the IEEE Congress on Evolutionary Computation, May 2015, pp. 1242-1249.

[10] N. Bacanin, M. Tuba, and B. Pelevic, "Constrained portfolio selection using artificial bee colony (ABC) algorithm", Optimization, vol. 17, pp. 2831-2844, 2014.

[11] J. E. Beasley, "OR-Library: Distributing test problems by electronic mail", Journal of the Operational Research Society, vol. 41, no. 11, pp. 1069-1072, Nov. 1990, ISSN: 1476-9360. 
[12] S. A. Bin Shalan and M. Ykhlef, "Solving multi-objective portfolio optimization problem for Saudi Arabia stock market using hybrid clonal selection and particle swarm optimization", Arabian Journal for Science and Engineering, vol. 40, no. 8, pp. 2407-2421, Aug. 2015, ISSN: 2191-4281.

[13] J. Bock and J. Hettenhausen, "Discrete particle swarm optimisation for ontology alignment", Information Sciences, vol. 192, pp. 152-173, 2012.

[14] J. Branke, B. Scheckenbach, M. Stein, K. Deb, and H. Schmeck, "Portfolio optimization with an envelope-based multi-objective evolutionary algorithm", European Journal of Operational Research, vol. 199, no. 3, pp. 684-693, 2009, ISSN: 0377-2217.

[15] F. Busetti, "Heuristic approaches to realistic portfolio optimisation", WIT Transactions on Modelling and Simulation, vol. 43, 2006.

[16] T. G. Carolus and A. P. Engelbrecht, "Control parameter importance and sensitivity analysis of the multi-guide particle swarm optimization algorithm", in Proceedings of the Swarm Intelligence; and in Lecture Notes in Computer Science, Cham: Springer International Publishing, 2020, pp. 96-106, ISBN: 978-3-030-60376-2.

[17] J. Chang, T. Tsaur, and J. Huang, "Applying investment satisfied capability index and genetic algorithms to construct the stocks portfolio", in Proceedings of the 3rd International Conference on Innovative Computing Information and Control, Jun. 2008, pp. 265-265.

[18] T. Chang, N. Meade, J. Beasley, and Y. Sharaiha, "Heuristics for cardinality constrained portfolio optimisation", Computers 83 Operations Research, vol. 27 , no. 13 , pp. 1271-1302, 2000, ISSN: 0305-0548.

[19] T.-J. Chang, S.-C. Yang, and K.-J. Chang, "Portfolio optimization problems in different risk measures using genetic algorithm", Expert Systems with Applications, vol. 36, no. 7, pp. $10529-10537$, 2009, ISSN: 0957-4174.

[20] A. H. L. Chen, Y. Liang, and C. Liu, "An artificial bee colony algorithm for the cardinality-constrained portfolio optimization problems", in Proceedings of the IEEE Congress on Evolutionary Computation, Jun. 2012, pp. 29022909.

[21] _ - "Portfolio optimization using improved artificial bee colony approach", in Proceedings of the IEEE Conference on Computational Intelligence for Financial Engineering and Economics, Apr. 2013, pp. 60-67.

[22] C. Chen and B.-Y. Chen, "Complex portfolio selection using improved particle swarm optimization approach", in Proceedings of the IEEE 20th International Conference on High Performance Computing and Communications, Jun. 2018, pp. 828-835.

[23] W. Chen, R. Zhang, Y. Cai, and F. Xu, "Particle swarm optimization for constrained portfolio selection problems", in Proceedings of the International Conference on Machine Learning and Cybernetics, Aug. 2006, pp. 2425-2429. 
[24] W. Chen and W.-G. Zhang, "The admissible portfolio selection problem with transaction costs and an improved PSO algorithm", Physica A: Statistical Mechanics and its Applications, vol. 389, no. 10, pp. 2070-2076, 2010, ISSN: 0378-4371.

[25] W.-N. Chen, J. Zhang, H. Chung, W.-L. Zhong, W.-G. Wu, and Y.-h. Shi, "A novel set-based particle swarm optimization method for discrete optimization problems", IEEE Transactions on Evolutionary Computation, vol. 14, no. 2, pp. 278-300, 2010.

[26] S. C. Chiam, A. Al Mamun, and Y. L. Low, "A realistic approach to evolutionary multi-objective portfolio optimization", in Proceedings of the IEEE Congress on Evolutionary Computation, Sep. 2007, pp. 204-211.

[27] S. C. Chiam, K. C. Tan, and A. Al Mamum, "Evolutionary multi-objective portfolio optimization in practical context", International Journal of Automation and Computing, vol. 5, no. 1, pp. 67-80, Jan. 2008, ISSN: 1751-8520.

[28] C.-Y. ChiangLin, "Applications of genetic algorithm to portfolio optimization with practical transaction constraints", in Proceedings of the 9th Joint International Conference on Information Sciences, Atlantis Press, 2006, ISBN: 978-90-78677-01-7.

[29] S.-C. Chu and P.-W. Tsai, "Computational intelligence based on the behavior of cats", International Journal of Innovative Computing, Information and Control, vol. 3, no. 1, pp. 163-173, 2007.

[30] L.-Y. Chuang, S.-W. Tsai, and C.-H. Yang, "Catfish particle swarm optimization", in Proceedings of the IEEE Swarm Intelligence Symposium, 2008, pp. 15.

[31] — - "Improved binary particle swarm optimization using catfish effect for feature selection", Expert Systems With Applications, vol. 38, no. 10, pp. 12699 $12707,2011$.

[32] C. W. Cleghorn and A. P. Engelbrecht, "Particle swarm convergence: An empirical investigation", Proceedings of the IEEE Congress of Evolutionary Computation, vol. 1, pp. 2524-2530, 2014.

[33] _ - "Particle swarm stability: A theoretical extension using the non-stagnate distribution assumption", Swarm Intelligence, vol. 12, no. 1, pp. 1-22, 2018.

[34] C. A. C. Coello Coello, "An introduction to multi-objective particle swarm optimizers", in Soft computing in industrial applications, Springer, 2011, pp. 312.

[35] C. A. C. Coello Coello, G. B. Lamont, and D. A. Van Veldhuizen, Evolutionary algorithms for solving multi-objective problems. Springer, 2007, vol. 5.

[36] C. A. C. Coello Coello and M. S. Lechuga, "MOPSO: A proposal for multiple objective particle swarm optimization", in Proceedings of the IEEE Congress on Evolutionary Computation, vol. 2, 2002, pp. 1051-1056. 
[37] C. A. C. Coello Coello and M. Reyes Sierra, "A Study of the Parallelization of a Coevolutionary Multi-objective Evolutionary Algorithm", in Proceedings of the Advances in Artificial Intelligence, Berlin, Heidelberg: Springer Berlin Heidelberg, 2004, pp. 688-697, ISBN: 978-3-540-24694-7.

[38] M. Corazza, G. Fasano, and R. Gusso, "Particle swarm optimization with non-smooth penalty reformulation, for a complex portfolio selection problem", Applied Mathematics and Computation, vol. 224, pp. 611-624, 2013, ISSN: 0096-3003.

[39] E. S. Correa, A. A. Freitas, and C. G. Johnson, "A new discrete particle swarm algorithm applied to attribute selection in a bioinformatics data set", in Proceedings of the 8th Annual Conference on Genetic and Evolutionary Computation, 2006, pp. 35-42.

[40] T. Cura, "Particle swarm optimization approach to portfolio optimization", Nonlinear Analysis: Real World Applications, vol. 10, no. 4, pp. 2396-2406, 2009, ISSN: $1468-1218$.

[41] K. Deb, A. Pratap, S. Agarwal, and T. Meyarivan, "A fast and elitist multiobjective genetic algorithm: NSGA-II", IEEE Transactions on Evolutionary Computation, vol. 6, no. 2, pp. 182-197, 2002.

[42] K. Deb, R. E. Steuer, R. Tewari, and R. Tewari, "Bi-objective portfolio optimization using a customized hybrid NSGA-II procedure", in Proceedings of the Evolutionary Multi-Criterion Optimization Conference and Lecture Notes in Computer Science, Berlin, Heidelberg: Springer Berlin Heidelberg, 2011, pp. 358-373, ISBN: 978-3-642-19893-9.

[43] G.-F. Deng and W.-T. Lin, "Ant colony optimization for Markowitz meanvariance portfolio model", in Proceedings of the Swarm, Evolutionary, and Memetic Computing, Berlin, Heidelberg: Springer Berlin Heidelberg, 2010, pp. 238-245.

[44] G.-F. Deng, W.-T. Lin, and C.-C. Lo, "Markowitz-based portfolio selection with cardinality constraints using improved particle swarm optimization", $E x$ pert Systems with Applications, vol. 39, no. 4, pp. 4558-4566, 2012, ISSN: 0957-4174.

[45] L. Diosan, "A multi-objective evolutionary approach to the portfolio optimization problem", in Proceedings of the International Conference on Computational Intelligence for Modelling, Control and Automation and International Conference on Intelligent Agents, Web Technologies and Internet Commerce, vol. 2, Nov. 2005, pp. 183-187.

[46] K. Doerner, W. J. Gutjahr, R. F. Hartl, C. Strauss, and C. Stummer, "Pareto ant colony optimization: A metaheuristic approach to multi-objective portfolio selection", Annals of Operations Research, vol. 131, no. 1, pp. 79-99, Oct. 2004, ISSN: $1572-9338$.

[47] M. Dorigo and G. Di Caro, "Ant colony optimization: A new meta-heuristic", in Proceedings of the IEEE Congress on Evolutionary Computation, vol. 2, Jul. 1999, 1470-1477 Vol. 2. 
[48] R. Drezewski and K. Doroz, "An agent-based co-evolutionary multi-objective algorithm for portfolio optimization", Symmetry, vol. 9, no. 9, 2017, ISSN: 2073-8994.

[49] R. Eberhart and J. Kennedy, "A new optimizer using particle swarm theory", in Proceedings of the Sixth International Symposium on Micro Machine and Human Science, Oct. 1995, pp. 39-43.

[50] M. Ehrgott, K. Klamroth, and C. Schwehm, "An MCDM approach to portfolio optimization", European Journal of Operational Research, vol. 155, no. 3, pp. 752-770, 2004, ISSN: 377-2217.

[51] A. Engelbrecht, "Particle swarm optimization: Global best or local best?", in Proceedings of the BRICS Congress on Computational Intelligence and 11th Brazilian Congress on Computational Intelligence, Sep. 2013, pp. 124-135.

[52] A. P. Engelbrecht, Computational Intelligence - An Introduction (2. ed.) Wiley, 2007, ISBN: 978-0-470-03561-0.

[60] S. Farzi, A. R. Shavazi, A. Pandari, and M. Graduated, "Using quantumbehaved particle swarm optimization for portfolio selection problem", International Arab Journal of Information Technology, vol. 10, no. 2, pp. 111-119, 2013.

[61] Fasheng $\mathrm{Xu}$ and Wei Chen, "Stochastic portfolio selection based on velocity limited particle swarm optimization", in Proceedings of the 6th World Congress on Intelligent Control and Automation, vol. 1, Jun. 2006, pp. 35993603.

[62] N. Franken, "Visual exploration of algorithm parameter space", in Proceedings of the IEEE Congress on Evolutionary Computation, 2009, pp. 389-398.

[63] A. Fraser., "Simulation of genetic systems by automatic digital computers I: Introduction", Australian Journal of Biological Sciences, vol. 11, no. 4, pp. 603-612, 1957.

[64] _ - "Simulation of genetic systems by automatic digital computers II: Effects of linkage on rates of advance under selection", Australian Journal of Biological Sciences, vol. 10, no. 4, pp. 492-500, 1957.

[65] A. H. Gandomi and A. H. Alavi, "Krill herd: A new bio-inspired optimization algorithm", Communications in Nonlinear Science and Numerical Simulation, vol. 17, no. 12 , pp. 4831-4845, 2012, ISSN: 1007-5704.

[66] M. Ge, "Artificial bee colony algorithm for portfolio optimization", in Proceedings of the International Conference on Intelligent Control and Information Processing, Aug. 2014, pp. 449-453.

[67] H. R. Golmakani and M. Fazel, "Constrained portfolio selection using particle swarm optimization", Expert Systems with Applications, vol. 38, no. 7, pp. 8327-8335, 2011, ISSN: 0957-4174.

[68] A. Gorgulho, R. F. Neves, and N. Horta, Intelligent financial portfolio composition based on evolutionary computation strategies. Springer Science \& Business Media, 2012. 
[69] B. Graham, The Intelligent Investor, Revised Edition. HarperCollins e-books, 2009.

[70] S. Guo, L. Yu, X. Li, and S. Kar, "Fuzzy multi-period portfolio selection with different investment horizons", European Journal of Operational Research, vol. 254 , no. 3 , pp. 1026-1035, 2016, ISSN: 0377-2217.

[71] A. S. Gyamerah, "Heuristic crossover for portfolio selection", Applied Mathematical Sciences, vol. 8, no. 65, pp. 3215-3227, 2014.

[72] A. S. Hadi, A. A. El Naggar, and M. N. A. Bary, "New model and method for portfolios selection", Applied Mathematical Sciences, vol. 10, no. 5-8, pp. 263$288,2016$.

[73] R. W. Hamming, "Error detecting and error correcting codes", The Bell System Technical Journal, vol. 29, no. 2, pp. 147-160, 1950.

[74] N. Hansen, "Adaptive encoding: How to render search coordinate system invariant", in Proceedings of the International Conference on Parallel Problem Solving from Nature, Springer, 2008, pp. 205-214.

[75] F.-F. Hao and Y.-K. Liu, "Mean-variance models for portfolio selection with fuzzy random returns", Journal of Applied Mathematics and Computing, vol. 30, no. 1 , pp. 9-38, Sep. 2008, ISSN: 1865-2085.

[76] Hoklie and L. R. Zuhal, "Resolving multi objective stock portfolio optimization problem using genetic algorithm", in Proceedings of the 2nd International Conference on Computer and Automation Engineering, vol. 2, Feb. 2010, pp. 40-44.

[77] L. Hong-Mei, W. Zhuo-Fu, and L. Hui-Min, "Artificial bee colony algorithm for real estate portfolio optimization based on risk preference coefficient", in Proceedings of the International Conference on Management Science Engineering, Nov. 2010, pp. 1682-1687.

[78] V. L. Huang, P. N. Suganthan, and J. J. Liang, "Comprehensive learning particle swarm optimizer for solving multi-objective optimization problems", International Journal of Intelligent Systems, vol. 21, no. 2, pp. 209-226, 2006.

[79] X. Huang and W. Shen, "Multi-period mean-variance model with transaction cost for fuzzy portfolio selection", in Proceedings of the 7th International Conference on Fuzzy Systems and Knowledge Discovery, vol. 2, Aug. 2010, pp. 894-898.

[80] X. Huang, "Mean-âvariance models for portfolio selection subject to experts estimations", Expert Systems with Applications, vol. 39, no. 5, pp. 5887-5893, 2012, ISSN: 0957-4174.

[81] S. Huband, P. Hingston, L. Barone, and L. While, "A review of multiobjective test problems and a scalable test problem toolkit", IEEE Transactions on Evolutionary Computation, vol. 10, no. 5, 477âa-506, 2006.

[82] H. Ishibuchi, N. Tsukamoto, and Y. Nojima, "Evolutionary many-objective optimization: A short review", in Proceedings of the IEEE Congress on Evolutionary Computation, Jun. 2008, pp. 2419-2426. 
[83] P. Jaccard, "Etude comparative de la distribution florale dans une portion des Alpes et des Jura", Bulletin de la Socieİteİ vaudoise des sciences naturelles, vol. 37 , pp. 547-579, 1901.

[84] H. Jalota and M. Thakur, "Genetic algorithm designed for solving portfolio optimization problems subjected to cardinality constraint", International Journal of System Assurance Engineering and Management, vol. 9, no. 1, pp. 294-305, Feb. 2018, ISSN: 0976-4348.

[85] H. John, Adaptation in natural and artificial systems. MIT Press, Cambridge, 1992.

[86] C. B. Kalayci, O. Ertenlice, and M. A. Akbay, "A comprehensive review of deterministic models and applications for mean-variance portfolio optimization", Expert Systems with Applications, vol. 125, pp. 345-368, 2019.

[87] C. B. Kalayci, O. Ertenlice, H. Akyer, and H. Aygoren, "An artificial bee colony algorithm with feasibility enforcement and infeasibility toleration procedures for cardinality constrained portfolio optimization", Expert Systems with Applications, vol. 85, pp. 61-75, 2017, ISSN: 0957-4174.

[88] S. Kamali, "Portfolio optimization using particle swarm optimization and genetic algorithm", Journal of Mathematics and Computer Science, vol. 10, pp. 85-90, 2014.

[89] H. Kamili and M. E. Riffi, "A comparative study on portfolio optimization problem", in Proceedings of the International Conference on Engineering, Sep. 2016, pp. 1-8.

[90] _ _ "Portfolio selection using the cat swarm optimization", Journal of Theoretical \&5 Applied Information Technology, vol. 74, no. 3, pp. 374-380, 2015.

[91] Y. Kao and H.-T. Cheng, "Bacterial foraging optimization approach to portfolio optimization", Computational Economics, vol. 42, no. 4, pp. 453-470, Dec. 2013, ISSN: 1572-9974.

[92] D. Karaboga, "An idea based on honey bee swarm for numerical optimization", Erciyes University, Engineering Faculty, Tech. Rep. 6, 2005.

[93] M. Kaucic, "Equity portfolio management with cardinality constraints and risk parity control using multi-objective particle swarm optimization", Computers 63 Operations Research, vol. 109, pp. 300-316, 2019, ISSN: 0305-0548.

[94] J. Kennedy and R. C. Eberhart, "A discrete binary version of the particle swarm algorithm", in Proceedings of the IEEE International Conference on Systems, Man, and Cybernetics, vol. 5, Oct. 1997, pp. 4104-4108.

[95] _ _ "A discrete binary version of the particle swarm algorithm", in Proceedings of the IEEE International Conference on Systems, Man, and Cybernetics. Computational Cybernetics and Simulation, vol. 5, 1997, pp. 4104-4108.

[96] M. Khalidji, M. Zeiaee, A. Taei, M. R. Jahed-Motlagh, and H. Khaloozadeh, "Dynamically weighted continuous ant colony optimization for bi-objective portfolio selection using value-at-risk", in Proceedings of the 3rd Asia International Conference on Modelling Simulation, May 2009, pp. 230-235. 
[97] S. A. Khan and A. P. Engelbrecht, "A fuzzy particle swarm optimization algorithm for computer communication network topology design", Applied Intelligence, vol. 36, no. 1, pp. 161-177, 2012.

[98] — - "A new fuzzy operator and its application to topology design of distributed local area networks", Information Sciences, vol. 177, no. 13, pp. 26922711, 2007.

[99] M. Koshino, H. Murata, and H. Kimura, "Improved particle swarm optimization and application to portfolio selection", Electronics and Communications in Japan (Part III: Fundamental Electronic Science), vol. 90, no. 3, pp. 13-25, 2007.

[100] D. Kumar and K. Mishra, "Portfolio optimization using novel co-variance guided artificial bee colony algorithm", Swarm and Evolutionary Computation, vol. 33, pp. 119-130, 2017, ISSN: 2210-6502.

[101] D. E. Kvasov and M. S. Mukhametzhanov, "Metaheuristic vs. deterministic global optimization algorithms: The univariate case", Applied Mathematics and Computation, vol. 318, pp. 245-259, 2018, ISSN: 0096-3003.

[103] A. Y. S. Lam and V. O. K. Li, "Chemical reaction inspired metaheuristic for optimization", IEEE Transactions on Evolutionary Computation, vol. 14, no. 3, pp. 381-399, Jun. 2010.

[104] J. Langeveld and A. P. P. Engelbrecht, "Set-based particle swarm optimization applied to the multidimensional knapsack problem", Swarm Intelligence, vol. 6, no. 4, pp. 297-342, 2012.

[105] J. Langeveld and A. P. Engelbrecht, "A generic set-based particle swarm optimization algorithm", International Conference on Swarm Intelligence, pp. 110, Jan. 2011.

[106] X. Li, "A non-dominated sorting particle swarm optimizer for multi-objective optimization", in Proceedings of the Genetic and Evolutionary Computation Conference, Springer, 2003, pp. 37-48.

[107] K. Liagkouras and K. Metaxiotis, "A new probe guided mutation operator and its application for solving the cardinality constrained portfolio optimization problem", Expert Systems with Applications, vol. 41, no. 14, pp. 6274-6290, 2014, ISSN: 0957-4174.

[108] K. Liagkouras, "A new three-dimensional encoding multi-objective evolutionary algorithm with application to the portfolio optimization problem", Knowledge-Based Systems, vol. 163, pp. 186-203, 2018, ISSN: 0950-7051.

[109] J. J. Liang and B. Y. Qu, "Large-scale portfolio optimization using multiobjective dynamic mutli-swarm particle swarm optimizer", in Proceedings of the IEEE Symposium on Swarm Intelligence, Apr. 2013, pp. 1-6.

[110] J. Liu, X. Jin, T. Wang, and Y. Yuan, "Robust multi-period portfolio model based on prospect theory and ALMV-PSO algorithm", Expert Systems with Applications, vol. 42, no. 20, pp. 7252-7262, 2015, ISSN: 0957-4174. 
[111] Y. Liu and K. Passino, "Biomimicry of social foraging bacteria for distributed optimization: models, principles, and emergent behaviors", Journal of Optimization Theory and Applications, vol. 115, no. 3, pp. 603-628, Dec. 2002, ISSN: $1573-2878$.

[112] Y. J. Liu, W. G. Zhang, and W. J. XU, "Fuzzy multiple criteria portfolio selection optimization model under real constraints", Systems EngineeringTheory 83 Practice, vol. 10, no. 33, pp. 2462-2470, 2013.

[113] I. Loshchilov, M. Schoenauer, and M. Sebag, "Adaptive coordinate descent", in Proceedings of the 13th Annual Conference on Genetic and Evolutionary Computation, 2011, pp. 885-892.

[114] K. Lwin and R. Qu, "A hybrid algorithm for constrained portfolio selection problems", Applied Intelligence, vol. 39, no. 2, pp. 251-266, Sep. 2013, ISSN: 1573-7497.

[115] H. Markowitz, "Portfolio selection", The Journal of Finance, vol. 7, no. 1, pp. 77-91, 1952.

[116] S. S. Meghwani and M. Thakur, "Multi-objective heuristic algorithms for practical portfolio optimization and rebalancing with transaction cost", $A p$ plied Soft Computing, vol. 67, pp. 865-894, 2018, ISSN: 1568-4946.

[117] A. Mehrabian and C. Lucas, "A novel numerical optimization algorithm inspired from weed colonization", Ecological Informatics, vol. 1, no. 4, pp. 355366, 2006, ISSN: 1574-9541.

[118] K. Metaxiotis and K. Liagkouras, "Multiobjective evolutionary algorithms for portfolio management: A comprehensive literature review", Expert Systems with Applications, vol. 39, no. 14, pp. 11 685-11 698, 2012, ISSN: 0957-4174.

[119] S. K. Mishra, G. Panda, and S. Meher, "Multi-objective particle swarm optimization approach to portfolio optimization", in Proceedings of the World Congress on Nature and Biologically Inspired Computing, Dec. 2009, pp. 1612 1615.

[120] _ - "Prediction based mean-variance model for constrained portfolio assets selection using multi-objective evolutionary algorithms", Swarm and Evolutionary Computation, vol. 28, pp. 117-130, 2016, ISSN: 2210-6502.

[121] S. K. Mishra, "Robust and constrained portfolio optimization using multiobjective evolutionary algorithms", Ph.D. dissertation, National Institute of Technology Rourkela, 2012.

[122] S. K. Mishra, G. Panda, and R. Majhi, "Constrained portfolio asset selection using multi-objective bacteria foraging optimization", Operational Research, vol. 14, no. 1, pp. 113-145, Apr. 2014, ISSN: 1866-1505.

[123] R. Moral-Escudero, R. Ruiz-Torrubiano, and A. Suarez, "Selection of optimal investment portfolios with cardinality constraints", in Proceedings of the IEEE International Conference on Evolutionary Computation, Jul. 2006, pp. 23822388 . 
[124] __ "Selection of optimal investment portfolios with cardinality constraints", in Proceedings of the IEEE International Conference on Evolutionary Computation, Jul. 2006, pp. 2382-2388.

[125] S. Mostaghim and J. Teich, "Strategies for finding good local guides in multiobjective particle swarm optimization (MOPSO)", in Proceedings of the IEEE Swarm Intelligence Symposium), IEEE, 2003, pp. 26-33.

[126] M. Mozafari, F. Jolai, and S. Tafazzoli, "A new IPSO-SA approach for cardinality constrained portfolio optimization", International Journal of Industrial Engineering Computations, vol. 2, no. 2, pp. 249-262, 2011.

[127] M. Neethling and A. P. Engelbrecht, "Determining RNA secondary structure using set-based particle swarm optimization", in Proceedings of the IEEE International Conference on Evolutionary Computation, 2006, pp. 1670-1677.

[128] A. Nel and A. P. Engelbrecht, "Set-based particle swarm optimisation approach to training a support vector machine", under review, 2021.

[129] S. Nematollahi and G. Manzi, "Portfolio management using prospect theory: Comparing genetic algorithms and particle swarm optimization", Department of Economics, Management and Quantitative Methods at Universita degli Studi di Milano, Tech. Rep. 3, Mar. 2018. [Online]. Available: https : // ideas.repec.org/p/mil/wpdepa/2018-03.html.

[130] Q. Ni, X. Yin, K. Tian, and Y. Zhai, "Particle swarm optimization with dynamic random population topology strategies for a generalized portfolio selection problem", Natural Computing, vol. 16, no. 1, pp. 31-44, Mar. 2017, ISSN: $1572-9796$.

[131] B. Niu, Y. Fan, H. Xiao, and B. Xue, "Bacterial foraging based approaches to portfolio optimization with liquidity risk", Neurocomputing, vol. 98, pp. 90100, 2012, ISSN: 0925-2312.

[132] B. Niu, B. Xue, L. Li, and Y. Chai, "Symbiotic multi-swarm pso for portfolio optimization", in Proceedings of the Emerging Intelligent Computing Technology and Applications With Aspects of Artificial Intelligence, Berlin, Heidelberg: Springer Berlin Heidelberg, 2009, pp. 776-784, ISBN: 978-3-642-04020-7.

[133] E. Okkes and K. C.B., "A survey of swarm intelligence for portfolio optimization: Algorithms and applications", Swarm and evolutionary computation, vol. 39, pp. 36-52, 2018.

[134] O. Olorunda and A. P. Engelbrecht, "Measuring exploration/exploitation in particle swarms using swarm diversity", in Proceedings of the IEEE Congress on Evolutionary Computation, 2008, pp. 1128-1134.

[135] G. A. V. Pai, "Active portfolio rebalancing using multi-objective metaheuristics", in Proceedings of the IEEE Symposium Series on Computational Intelligence, 2018, pp. 1845-1852.

[136] —_, "Multi-objective Metaheuristics for Managing Futures Portfolio Risk", in Proceedings of the IEEE Symposium Series on Computational Intelligence, 2018, pp. 1204-1211. 
[137] G. A. V. Pai and T. Michel, "Differential evolution based optimization of risk budgeted Equity Market Neutral Portfolios", in Proceedings of the IEEE Congress on Evolutionary Computation, 2012, pp. 1-8.

[138] _ _ "Evolutionary optimization of constrained $k$-means clustered assets for diversification in small portfolios", IEEE Transactions on Evolutionary Computation, vol. 13, no. 5, pp. 1030-1053, Oct. 2009, ISSN: 1089-778X.

[139] —_, "Integrated metaheuristic optimization of 130-30 investment-strategybased long-short portfolios", International Journal of Intelligent Systems in Accounting, Finance \&3 Management, vol. 19, no. 1, pp. 43-74, 2012.

[140] —_, "Metaheuristic multi-objective optimization of constrained futures portfolios for effective risk management", Swarm and Evolutionary Computation, vol. 19, pp. 1-14, 2014.

[141] G. Pampara, A. Engelbrecht, and T. Cloete, "CIlib: A collaborative framework for computational intelligence algorithms - Part I", in Proceedings of the IEEE International Joint Conference on Neural Networks, 2008, pp. 17501757.

[142] K. E. Parsopoulos and M. N. Vrahatis, Particle swarm optimization and intelligence: Advances and applications. IGI global, 2010.

[143] — - "Recent approaches to global optimization problems through particle swarm optimization", Natural Computing, vol. 1, no. 2, pp. 235-306, Jun. 2002, ISSN: 1572-9796.

[144] A. R. Pouya, M. Solimanpur, and M. J. Rezaee, "Solving multi-objective portfolio optimization problem using invasive weed optimization", Swarm and Evolutionary Computation, vol. 28, pp. 42-57, 2016, ISSN: 2210-6502.

[145] G. T. Pulido and C. A. C. Coello Coello, "Using clustering techniques to improve the performance of a multi-objective particle swarm optimizer", in Proceedings of the Genetic and Evolutionary Computation Conference, Springer, 2004, pp. 225-237.

[146] B. Qu, Q. Zhou, J. Xiao, J. Liang, and P. N. Suganthan, "Large-scale portfolio optimization using multi-objective evolutionary algorithms and preselection methods", Mathematical Problems in Engineering, vol. 2017, pp. 1-14, 2017.

[147] R. Ruiz-Torrubiano and A. Suarez, "A memetic algorithm for cardinalityconstrained portfolio optimization with transaction costs", Applied Soft Computing, vol. 36, pp. 125-142, 2015, ISSN: 1568-4946.

[148] —_, "Hybrid approaches and dimensionality reduction for portfolio selection with cardinality constraints", IEEE Computational Intelligence Magazine, vol. 5, no. 2, pp. 92-107, May 2010, ISSN: 1556-603X.

[149] _ - "Use of heuristic rules in evolutionary methods for the selection of optimal investment portfolios", in Proceedings of the IEEE Congress on Evolutionary Computation, Sep. 2007, pp. 212-219. 
[150] A. N. Sadigh, H. Mokhtari, M. Iranpoor, and S. M. T. F. Ghomi, "Cardinality constrained portfolio optimization using a hybrid approach based on particle swarm optimization and hopfield neural network", Advanced Science Letters, vol. 17, no. 1, pp. 11-20, 2012, ISSN: 1936-6612.

[151] M. Salahi, M. Daemi, S. Lotfi, and A. Jamalian, "PSO and harmony search algorithms for cardinality constrained portfolio optimization problem", Advanced Modeling and Optimization, vol. 16, no. 3, pp. 559-573, Jan. 2014.

[152] C. Scheepers, C. Cleghorn, and A. P. Engelbrecht, "Multi-guide particle swarm optimization for multi-objective optimization: empirical and stability analysis", Swarm Intelligence, vol. 13, no. 2-3, pp. 245-276, 2019.

[153] Y. Shi and R. Eberhart, "A modified particle swarm optimizer", in Proceedings of the IEEE International Conference on Evolutionary Computation, 1998, pp. 69-73.

[154] J. Shoaf and J. A. Foster, "A genetic algorithm solution to the efficient set problem: A technique for portfolio selection based on the Markowitz model", in Proceedings of the Decision Sciences Institute Annual meeting, 1996, 571â573.

[155] —_, "The efficient set GA for stock portfolios", in Proceedings of the IEEE International Conference on Evolutionary Computation, May 1998, pp. 354359.

[156] M. R. Sierra and C. A. C. Coello Coello, "Improving PSO-based multiobjective optimization using crowding, mutation and dominance", in Proceedings of the International Conference on Evolutionary Multi-Criterion Optimization, Springer, 2005, pp. 505-519.

[157] Y. L. T. Silva, A. B. Herthel, and A. Subramanian, "A multi-objective evolutionary algorithm for a class of mean-variance portfolio selection problems", Expert Systems with Applications, vol. 133, pp. 225-241, 2019, ISSN: 09574174 .

[158] P. Skolpadungket, K. Dahal, and N. Harnpornchai, "Portfolio optimization using multi-objective genetic algorithms", in Proceedings of the IEEE Congress on Evolutionary Computation, Sep. 2007, pp. 516-523.

[159] H. Soleimani, H. R. Golmakani, and M. H. Salimi, "Markowitz-based portfolio selection with minimum transaction lots, cardinality constraints and regarding sector capitalization using genetic algorithm", Expert Systems with Applications, vol. 36, no. 3, Part 1, pp. 5058-5063, 2009, ISSN: 0957-4174.

[160] K. Sorensen, "Metaheuristics: The metaphor exposed", International Transactions in Operational Research, vol. 22, no. 1, pp. 3-18, 2015.

[161] R. Storn and K. Price, "Differential Evolution - A Simple and Efficient Heuristic for global Optimization over Continuous Spaces", Journal of Global Optimization, vol. 11, no. 4, pp. 341-359, Dec. 1997, ISSN: 1573-2916.

[162] F. Streichert and M. Tanaka-Yamawaki, "The effect of local search on the constrained portfolio selection problem", in Proceedings of the IEEE International Conference on Evolutionary Computation, Jul. 2006, pp. 2368-2374. 
[163] I. Strumberger, N. Bacanin, and M. Tuba, "Constrained portfolio optimization by hybridized bat algorithm", in Proceedings of the 7th International Conference on Intelligent Systems, Modelling and Simulation, Jan. 2016, pp. 8388.

[164] A. Stuart and H. M. Markowitz, "Portfolio selection: Efficient diversification of investments", A Quarterly Journal of Operations Research, vol. 10, no. 4, p. 253, 1959.

[165] R. Subbu, P. P. Bonissone, N. Eklund, S. Bollapragada, and K. Chalermkraivuth, "Multiobjective financial portfolio design: a hybrid evolutionary approach", in Proceedings of the IEEE Congress on Evolutionary Computation, vol. 2, Sep. 2005, pp. 1722-1729.

[166] J. Sun, W. Fang, X. Wu, C.-H. Lai, and W. Xu, "Solving the multi-stage portfolio optimization problem with a novel particle swarm optimization", Expert Systems with Applications, vol. 38, no. 6, pp. 6727-6735, 2011, ISSN: 0957-4174.

[167] D. Suthiwong and M. Sodanil, "Cardinality-constrained portfolio optimization using an improved quick artificial bee colony algorithm", in Proceedings of the International Computer Science and Engineering Conference, Dec. 2016, pp. 1-4.

[168] Y. Tan and Y. Zhu, "Fireworks algorithm for optimization", in Proceedings of the Advances in Swarm Intelligence, Berlin, Heidelberg: Springer Berlin Heidelberg, 2010, pp. 355-364, ISBN: 978-3-642-13495-1.

[169] P. K. Tripathy, S. Bandyopadhyay, and S. K. Pal, "Adaptive multi-objective particle swarm optimization algorithm", IEEE Congress on Evolutionary Computation, 2007.

[170] V. Trivedi, P. Varshney, and M. Ramteke, "A simplified multi-objective particle swarm optimization algorithm", Swarm Intelligence, vol. 14, no. 2, pp. 83116, 2019.

[171] S.-J. Tsai, T.-Y. Sun, C.-C. Liu, S.-T. Hsieh, W.-C. Wu, and S.-Y. Chiu, "An improved multi-objective particle swarm optimizer for multi-objective problems", Expert Systems with Applications, vol. 37, no. 8, pp. 5872-5886, 2010, ISSN: 0957-4174.

[172] M. Tuba and N. Bacanin, "Artificial bee colony algorithm hybridized with firefly algorithm for cardinality constrained mean-variance portfolio selection problem", Applied Mathematics 83 Information Sciences, vol. 8, no. 6, p. 2831, 2014.

[173] _ _ "Upgraded firefly algorithm for portfolio optimization problem", in Proceedings of the 16th International Conference on Computer Modelling and Simulation, Mar. 2014, pp. 113-118.

[174] M. Tuba, N. Bacanin, and B. Pelevic, "Krill herd (KH) algorithm applied to the constrained portfolio selection problem", Optimization, vol. 21, p. 22, 2014 . 
[175] D. A. Van Veldhuizen and G. B. Lamont, "Multiobjective evolutionary algorithm research: A history and analysis", Department of Electrical and Computer Engineering, Tech. Rep., 1998.

[176] __, "On measuring multi-objective evolutionary algorithm performance", in Proceedings of the Congress on Evolutionary Computation, vol. 1, Jul. 2000, 204-211 vol.1.

[177] C. B. Veenhuis, "A set-based particle swarm optimization method", in Proceedings of the 10th International Conference on Parallel Problem Solving, 2008, pp. 971-980.

[178] B. Wang and J. Watada, "Multi-objective particle swarm optimization for a novel fuzzy portfolio selection problem", IEEE Transactions on Electrical and Electronic Engineering, vol. 8, no. 2, pp. 146-154, 2013.

[179] L. Wang, X. Wang, J. Fu, and L. Zhen, "A novel probability binary particle swarm optimization algorithm and its application", Journal of Software, vol. 3, no. 9 , pp. 28-35, 2008.

[180] W. Wang, H. Wang, Z. Wu, and H. Dai, "A simple and fast particle swarm optimization and its application on portfolio selection", in Proceedings of the International Workshop on Intelligent Systems and Applications, May 2009, pp. $1-4$.

[181] Z. Wang, S. Liu, and X. Kong, "Artificial bee colony algorithm for portfolio optimization problems", International Journal of Advancements in Computing Technology, vol. 4, no. 4, pp. 8-16, 2012.

[182] D. Wolpert and W. Mac Ready, "No free lunch theorems for optimization", IEEE Transactions on Evolutionary Computation, vol. 1, no. 1, pp. 67-82, 1997.

[183] M. Woodside-Oriakhi, C. Lucas, and J. Beasley, "Heuristic algorithms for the cardinality constrained efficient frontier", European Journal of Operational Research, vol. 213, no. 3, pp. 538-550, 2011, ISSN: 0377-2217.

[184] J. Xu, A. Y. S. Lam, and V. O. K. Li, "Stock portfolio selection using chemical reaction optimization", International Journal of Chemical and Molecular Engineering, vol. 5, no. 5, pp. 409-414, 2011.

[185] S. B. Yaakob and J. Watada, "A hybrid particle swarm optimization approach to mixed integer quadratic programming for portfolio selection problems", International Journal of Simulation: Systems, Science and Technology, vol. 11, pp. 68-74, 2010.

[186] X.-S. Yang, "A new metaheuristic bat-inspired algorithm", in Nature Inspired Cooperative Strategies for Optimization. Berlin, Heidelberg: Springer Berlin Heidelberg, 2010, pp. 65-74, ISBN: 978-3-642-12538-6.

[187] — - "Firefly algorithms for multimodal optimization", in Proceedings of the Stochastic Algorithms: Foundations and Applications, Berlin, Heidelberg: Springer Berlin Heidelberg, 2009, pp. 169-178, ISBN: 978-3-642-04944-6. 
[188] X. Yin, Q. Ni, and Y. Zhai, "A novel PSO for portfolio optimization based on heterogeneous multiple population strategy", in Proceedings of the IEEE Congress on Evolutionary Computation, May 2015, pp. 1196-1203.

[189] L. Yu, S. Wang, and K. K. Lai, "Portfolio optimization using evolutionary algorithms", in Reflexing Interfaces: The Complex Coevolution of Information Technology Ecosystems, IGI Global, 2008, pp. 235-245.

[190] X. Zhang, X. Zheng, R. Cheng, J. Qiu, and Y. Jin, "A competitive mechanism based multi-objective particle swarm optimizer with fast convergence", Information Sciences, vol. 427, pp. 63-76, 2018.

[191] J. Zheng, Q. Wu, and W. Song, "An improved particle swarm algorithm for solving nonlinear constrained optimization problems", in Proceedings of the 3rd International Conference on Natural Computation, vol. 4, Aug. 2007, pp. $112-117$.

[192] J. Zhou and J. Li, "An improved multi-objective particle swarm optimization for constrained portfolio selection model", in Proceedings of the 11th International Conference on Service Systems and Service Management, Jun. 2014, pp. 1-5.

[193] H. Zhu, Y. Chen, and K. Wang, "Swarm intelligence algorithms for portfolio optimization", in Proceedings of the Advances in Swarm Intelligence, Berlin, Heidelberg: Springer Berlin Heidelberg, 2010, pp. 306-313, ISBN: 978-3-64213495-1.

[194] H. Zhu, Y. Wang, K. Wang, and Y. Chen, "Particle swarm optimization (PSO) for the constrained portfolio optimization problem", Expert Systems with Applications, vol. 38, no. 8, pp. 10161-10169, 2011, ISSN: 0957-4174.

[195] E. Zitzler, L. Thiele, M. Laumanns, C. M. Fonseca, and V. G. da Fonseca, "Performance assessment of multi-objective optimizers: an analysis and review", IEEE Transactions on Evolutionary Computation, vol. 7, no. 2, pp. 117132, Apr. 2003, ISSN: 1089-778X.

[196] J. van Zyl and A. P. Engelbrecht, "Polynomial approximation using set-based particle swarm optimization", in Proceedings of the International Conference on Swarm Intelligence, 2021. 


\section{Appendices}




\title{
Appendix A
}

\section{Acronyms}

\author{
ABC artificial bee colony \\ ACD adaptive coordinate descent \\ ACO ant colony optimization \\ AE adaptive encoding \\ BA bat algorithm \\ BFA bacterial foraging algorithm \\ BPSO binary particle swarm optimization \\ CCPP cardinality constrained portfolio problem \\ CD coordinate descent \\ CFBPSO catfish binary particle swarm optimization \\ CFPSO catfish particle swarm optimization \\ CMA-ES covariance matrix adaptation evolution strategy \\ CRO chemical reaction optimization \\ CSO cat swarm optimization \\ CVaR conditional value-at-risk \\ DE differential evolution \\ E-MOEA envelope multi-objective genetic algorithm \\ EA evolutionary algorithm \\ FA firefly algorithm \\ FPSO fuzzy particle swarm optimization \\ FWA fireworks algorithm \\ GA genetic algorithm \\ GD generational distance \\ HV hypervolume \\ IGD inverted generational distance \\ IWO invasive weed optimization \\ KH krill Herd \\ MAD mean absolute deviation \\ MBPSO modified binary particle swarm optimization \\ MGPSO multi-guide particle swarm optimization
}


MGSBPSO multi-guide set-based particle swarm optimization

MODE multi-objective differential evolution

MOEA multi-objective evolutionary algorithm

MOEA/D multi-objective evolutionary algorithm based on decomposition

MOGA multi-objective genetic algorithm

MOO multi-objective optimization

MOOP multi-objective optimization problem

MOPSO multi-objective particle swarm optimization

NSGA II non-dominated sorting genetic algorithm II

OMOPSO optimized multi-objective particle swarm optimization

PBPSO probability binary particle swarm optimization

PCA principal component analysis

PESA Pareto envelope-based selection algorithm

PESA-II Pareto envelope-based selection algorithm II

POF Pareto-optimal front

POS Pareto-optimal solutions

PSFGA parallel single front genetic algorithm

PSO particle swarm optimization

SA simulated annealing

SBPSO set-based particle swarm optimization

SI swarm intelligence

SMPSO speed-constrained multi-objective particle swarm optimization

SPEA2 strength Pareto evolutionary algorithm 2

TS tabu search

VaR value-at-risk

VEGA vector evaluated genetic algorithm

VEPSO vector evaluated particle swarm optimization

WFG Walking Fish Group 


\title{
Appendix B \\ List of Symbols
}

\author{
$\alpha \quad$ controls a randomized move \\ $\bar{\sigma} \quad$ total risk \\ $\overline{d_{H}} \quad$ Hamming diversity \\ $\bar{d}_{J} \quad$ Jaccard diversity \\ $\beta_{i} \quad$ attractiveness of firefly $i$ \\ $\hat{\boldsymbol{a}}_{i} \quad$ archive guide of particle $i$ \\ $\hat{\boldsymbol{y}}_{i} \quad$ social guide of particle $i$ \\ $\boldsymbol{a}, \boldsymbol{b} \quad$ lower and upper bounds of the ACD search \\ $\boldsymbol{o}_{i} \quad$ neighboring food source to food source $i$ \\ $\boldsymbol{r}_{1}, \boldsymbol{r}_{2}, \boldsymbol{r}_{3}, \boldsymbol{r}_{4}$ uniform vectors with components in the range $[0,1]$ \\ $\boldsymbol{v}_{i} \quad$ velocity of particle $i$ \\ $\boldsymbol{x}$ decision vector \\ $\boldsymbol{x}_{i}(t) \quad$ decision vector $i$ at time step $t$ \\ $\boldsymbol{y}_{i} \quad$ cognitive guide of particle $i$ \\ $\boxplus \quad$ set-based operator for addition of a set-based velocity and a \\ set-based position \\ $\gamma_{i} \quad$ light absorption coefficient \\ $\hat{A}_{i} \quad$ archive guide of set-particle $i$ \\ $\hat{Y}_{i} \quad$ social guide of set-particle $i$
}


$$
\kappa_{i}
$$

$\lambda$

$\lambda_{c}$

$\lambda_{i}$

$\Omega$

$\omega$

$\ominus$

$\oplus$

$\otimes$

$<$

\section{$\tau$}

$\varepsilon, \delta$

$\varphi$

B

C

$$
c_{1}, c_{2}, c_{3}, c_{4}
$$

$c_{i}$

$d$

$d_{H}$

$$
d_{J}
$$

$e^{*}$

F

$f$

$g$

$I_{i}$ amount of nectar at food source $i$

risk-return trade-off

exploration balance coefficient of particle $i$

archive balance coefficient of particle $i$

search space of an optimization problem

set to bit vector transformation function

set-based operator for the difference between two set-based positions

set-based operator for the addition of two set-based velocities

set-based operator for the multiplication of a set-based velocity by a scalar value

Pareto-dominance relation

tournament size

lower and upper bounds of the asset weights

uniform vector with components in the range $[-1,1]$

total portfolio budget

benchmark to compare portfolio performance

positive acceleration coefficients

cost of asset $i$

number of dimensions in a search space

Hamming distance

Jaccard distance

minimum amount of liquidity

remaining free portion of the portfolio

objective function

uniform value in the range $[0,1]$

light intensity of firefly $i$ 


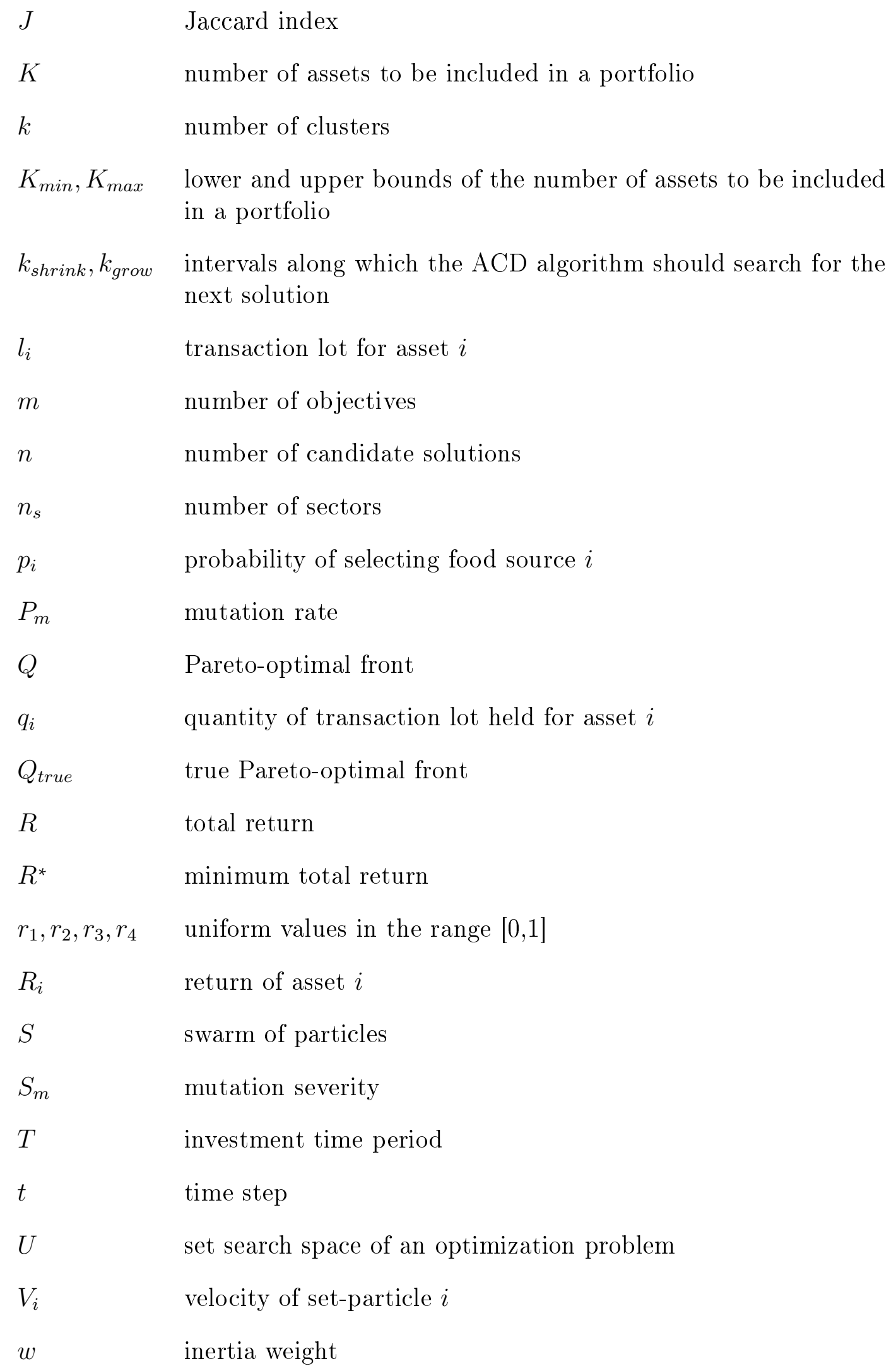


$w_{i} \quad$ weighting of asset $i$

$X(t) \quad$ position of set-particle $i$ at time step $t$

$Y_{i} \quad$ cognitive guide of set-particle $i$

$z_{i} \quad$ bit value indicating whether asset $i$ is included in the portfolio 


\section{Appendix C}

\section{Derived Publications}

This appendix lists publications derived from the work presented in this study in order of publication date:

- K. Erwin and A. P. Engelbrecht, "Set-Based particle swarm optimization for portfolio optimization", in Proceedings of the 12th International Swarm Intelligence Conference (ANTS); and in Lecture Notes in Computer Science, Springer International Publishing, 2020, pp. 333-339, ISBN: 978-3-030-60376-2

- K. Erwin and A. P. Engelbrecht, "Diversity measures for set-based metaheuristics", in Proceedings of the 7th International Conference on Soft Computing Machine Intelligence, 2020, pp. 45-50

- K. Erwin and A. P. Engelbrecht, "Improved set-based particle swarm optimization for portfolio optimization", in Proceedings of the IEEE Swarm Intelligence Symposium, 2020, pp. 1573-1580

- K. Erwin and A. P. Engelbrecht, "Evolutionary and swarm intelligence algorithms for portfolio optimization", Applied Soft Computing (under review), 2021

- Kyle and A. P. Engelbrecht, "Set-based particle swarm optimization for portfolio optimization: Empirical analysis.", Applied Soft Computing (under review), 2021

- K. Erwin and A. P. Engelbrecht, "Set-based particle swarm optimization for portfolio optimization with adaptive coordinate descent weight optimization", IEEE Swarm Intelligence Symposium (under review), 2021

- K. Erwin and A. P. Engelbrecht, "A tuning free approach to multi-guide particle swarm optimization", IEEE Swarm Intelligence Symposium (under review), 2021 
- K. Erwin and A. P. Engelbrecht, "Multi-guide set-based particle swarm optimization for portfolio optimization", IEEE Transactions on Evolutionary Computation (under review), 2021 


\section{Appendix D}

\section{Control Parameter Values}

Table D.1: Tuned control parameters for the Hang Seng benchmark problem for each algorithm

\begin{tabular}{lll}
\hline Algorithm & Parameter & Value \\
\hline ABC & limit & 97 \\
\hline FA & $\beta_{0}$ & 4.765625 \\
& $\gamma$ & 0.476563 \\
& $\alpha$ & 0.992188 \\
\hline GA & $S_{m}$ & 0.155469 \\
& $\tau$ & 11 \\
\hline PSO & $w$ & 0.937500 \\
& $c_{1}$ & 0.476563 \\
& $c_{2}$ & 0.992188 \\
\hline SBPSO & $c_{1}$ & 0.375000 \\
& $c_{2}$ & 0.375000 \\
& $c_{3}$ & 3.312500 \\
& $c_{4}$ & 4.437500 \\
\hline
\end{tabular}


Table D.2: Tuned control parameters for the DAX 100 benchmark problem for each algorithm

\begin{tabular}{lll}
\hline Algorithm & Parameter & Value \\
\hline ABC & limit & 98 \\
\hline FA & $\beta_{0}$ & 5.234375 \\
& $\gamma$ & 0.523438 \\
& $\alpha$ & 0.992188 \\
\hline GA & $S_{m}$ & 0.052109 \\
& $\tau$ & 13 \\
\hline PSO & $w$ & 0.851563 \\
& $c_{1}$ & 1.140625 \\
& $c_{2}$ & 1.234375 \\
\hline SBPSO & $c_{1}$ & 0.117188 \\
& $c_{2}$ & 0.117188 \\
& $c_{3}$ & 3.488281 \\
& $c_{4}$ & 2.714844 \\
\hline
\end{tabular}

Table D.3: Tuned control parameters for the FTSE 100 benchmark problem for each algorithm

\begin{tabular}{lll}
\hline Algorithm & Parameter & Value \\
\hline ABC & limit & 91 \\
\hline FA & $\beta_{0}$ & 8.593750 \\
& $\gamma$ & 0.859375 \\
& $\alpha$ & 0.921875 \\
\hline GA & $S_{m}$ & 0.017656 \\
& $\tau$ & 16 \\
\hline PSO & $w$ & 0.781250 \\
& $c_{1}$ & 1.562500 \\
& $c_{2}$ & 1.312500 \\
\hline SBPSO & $c_{1}$ & 0.367188 \\
& $c_{2}$ & 0.867188 \\
& $c_{3}$ & 2.363281 \\
& $c_{4}$ & 3.839844 \\
\hline & &
\end{tabular}


Table D.4: Tuned control parameters for the S\&P 100 benchmark problem for each algorithm

\begin{tabular}{lll}
\hline Algorithm & Parameter & Value \\
\hline ABC & limit & 96 \\
\hline FA & $\beta_{0}$ & 8.203125 \\
& $\gamma$ & 0.820313 \\
& $\alpha$ & 0.960938 \\
\hline GA & $S_{m}$ & 0.270313 \\
& $\tau$ & 3 \\
\hline PSO & $w$ & 0.781250 \\
& $c_{1}$ & 1.562500 \\
& $c_{2}$ & 1.312500 \\
\hline SBPSO & $c_{1}$ & 0.828125 \\
& $c_{2}$ & 0.484375 \\
& $c_{3}$ & 2.960938 \\
& $c_{4}$ & 2.257813 \\
\hline
\end{tabular}

Table D.5: Tuned control parameters for the Nikkei 225 benchmark problem for each algorithm

\begin{tabular}{lll}
\hline Algorithm & Parameter & Value \\
\hline ABC & limit & 97 \\
\hline FA & $\beta_{0}$ & 3.984375 \\
& $\gamma$ & 0.398438 \\
& $\alpha$ & 0.820313 \\
\hline GA & $S_{m}$ & 0.036797 \\
& $\tau$ & 19 \\
\hline PSO & $w$ & 0.851563 \\
& $c_{1}$ & 1.140625 \\
& $c_{2}$ & 1.234375 \\
\hline SBPSO & $c_{1}$ & 0.828125 \\
& $c_{2}$ & 0.484375 \\
& $c_{3}$ & 2.960938 \\
& $c_{4}$ & 2.257813 \\
\hline
\end{tabular}




\section{Appendix E}

\section{Walking Fish Group Toolkit}

Huband et al. proposed a sophisticated toolkit, referred to as the WFG toolkit, that contains nine scalable benchmarks with varying properties 81 . All problems created by the WFG toolkit conform to the following format:

$m$ is the number of objectives, $\boldsymbol{x}$ is a set of underlying parameters, where $\boldsymbol{x}_{m}$ is an underlying distance parameter and $x_{1: m-1}$ are underlying position parameters;

$\boldsymbol{z}$ is a set of $j+1=n \geq m$ working parameters, where the first $j$ and the last $l$ working parameters are position- and distance-related parameters, respectively;

$D>0$ is a distance scaling constant equal to one;

$A_{1: m-1} \in\{0,1\}$ are degeneracy constants, for each $A_{i}=0$, the dimensionality of the $\mathrm{POF}$ is reduced by one;

$h_{1}: m$ are shape functions;

$S_{1: m}>0$ are scaling constants and

$\boldsymbol{t}^{1: p}$ are transition vectors, where "↔" indicates that each transition vector is created from another vector via transformation functions;

The domain of all $z_{i} \in \boldsymbol{z}$ is $\left[0, z_{i, \max }\right]$, where all $z_{i, \max }>0$. Note that all $x_{i} \in \boldsymbol{x}$ will have domain [0,1].

\section{E.1 Shape functions}

The following five shape functions determine the nature of the POF, and map parameters with domain $[0,1]$ onto the range $[0,1]$. Furthermore, for all shape functions $x_{1}, \cdots, x_{m-1} \in[0,1], A, \alpha$ and $\beta$ are constants. The shape functions are 


\section{Linear}

$$
\begin{aligned}
\operatorname{linear}_{1}\left(x_{1}, \cdots, x_{m-1}\right) & =\prod_{i=1}^{m-1} x_{i} \\
\operatorname{linear}_{k=2: m-1}\left(x_{1}, \cdots, x_{m-1}\right) & =\left(\prod_{i=1}^{m-k} x_{i}\right)\left(1-x_{m-k+1}\right) \\
\operatorname{linear}_{m}\left(x_{1}, \cdots, x_{m-1}\right) & =1-x_{1}
\end{aligned}
$$

When $h_{k=1: m}=\operatorname{linear}_{j}$, the POF is a linear hyperplane, where $\sum_{k=1}^{m}=1$.

\section{Convex}

$$
\begin{aligned}
\operatorname{convex}_{1}\left(x_{1}, \cdots, x_{m-1}\right) & =\prod_{i=1}^{m-1}\left(1-\cos \left(x_{i} \frac{\pi}{2}\right)\right) \\
\operatorname{convex}_{k=2: m-1}\left(x_{1}, \cdots, x_{m-1}\right) & =\left(\prod_{i=1}^{m-k}\left(1-\cos \left(x_{i} \frac{\pi}{2}\right)\right)\right)\left(1-\sin \left(x_{m-k+1} \frac{\pi}{2}\right)\right) \\
\operatorname{convex}_{m}\left(x_{1}, \cdots, x_{m-1}\right) & =1-\sin \left(x_{1} \frac{\pi}{2}\right)
\end{aligned}
$$

When $h_{k=1: m}=\operatorname{convex}_{j}$, the POF is a is purely convex.

\section{Concave}

$$
\begin{aligned}
\operatorname{concave}_{1}\left(x_{1}, \cdots, x_{m-1}\right) & =\prod_{i=1}^{m-1}\left(1-\sin \left(x_{1} \frac{\pi}{2}\right)\right. \\
\text { concave }_{k=2: m-1}\left(x_{1}, \cdots, x_{m-1}\right) & =\left(\prod_{i=1}^{m-k}\left(\sin \left(x_{i} \frac{\pi}{2}\right)\right) \cos \left(x_{m-k+1} \frac{\pi}{2}\right)\right. \\
\text { concave }_{m}\left(x_{1}, \cdots, x_{m-1}\right) & =\cos \left(x_{1} \frac{\pi}{2}\right)
\end{aligned}
$$

When $h_{k=1: m}=$ concave $_{j}$, the POF is a is purely concave, and a region of the hyper-sphere of radius one centred at the origin, where $\sum_{k=1}^{m}=h_{k}^{2}=1$.

mixed convex/concave $(\alpha>0, A \in\{1,2, \cdots\})$

$$
\operatorname{mixed}_{m}\left(x_{1}, \cdots, x_{m-1}\right)=\left(1-x_{1}-\frac{\cos \left(2 A \pi x_{1}+\frac{\pi}{2}\right)}{2 A \pi}\right)^{\alpha}
$$

Equation E.5 causes the POF to contain both convex and concave segments, the number of which is controlled by $A$. The overall shape is controlled by $\alpha$ : when $\alpha>1$ or when $\alpha<1$, the overall shape is convex or concave, respectively. When $\alpha=1$, the overall shape in linear. 
Disconnected $(\alpha, \beta>0, A \in\{1,2, \cdots\})$

$$
\operatorname{disc}_{m}\left(x_{1}, \cdots, x_{m-1}\right)=1-\left(x_{1}\right)^{\alpha} \cos ^{2}\left(A\left(x_{1}\right)^{\beta} \pi\right)
$$

Equation (E.6) to have disconnected regions, the number of which is controlled by $A$. The overall shape is controlled by $\alpha$ : when $\alpha>1$ or when $\alpha<1$, the overall shape is convex or concave, respectively. When $\alpha=1$, the overall shape in linear. The location of the disconnected regions is controlled by $\beta$ : larger values of $\beta$ push the location of the disconnected regions towards larger values of $x_{1}$, and vice versa.

\section{E.2 Transformation functions}

Transformation functions map input parameters with domain $[0,1]$ onto the range $[0,1]$. A total of eight transformation functions are provided by the WFG toolkit and are categorised as bias, shift and reduction functions. The primary parameters $y$ and $y, \cdots, y_{|y| 3}$ always have domain of $[0,1]$. $A, B, C, \alpha$ and $\beta$ are constants.

\section{E.2.1 Bias Functions}

Bias transformations impact the search process by biasing the fitness landscape. The following bias transformations have been defined:

Polynomial $(\alpha>0, \alpha \neq 1)$

$$
\text { b_poly }(y, \alpha)=y^{\alpha}
$$

When $\alpha>1, y$ is biased towards zero, and when $\alpha<1, y$ is biased towards one.

Flat Region $(A, B, C \in[0,1], B<C, B=0 \Rightarrow A=0 \wedge C \neq 1, C=1 \Rightarrow A=$ $1 \wedge B \neq 0)$ )

$$
\begin{aligned}
& \text { b_flat }(y, A, B, C)=A+\min (0,\lfloor y-B\rfloor) \frac{A(B-y)}{B} \\
& -\min (0,\lfloor C-y\rfloor) \frac{(1-A)(y-C)}{(1-C)}
\end{aligned}
$$

Values of $y$ between $B$ and $C$, the area of the flat region, are all mapped to the value of $A$. 
Parameter Dependent $(A \in(0,1), 0<B<C)$

$$
\begin{aligned}
\text { b_param }\left(y, \boldsymbol{y}^{\prime}, A, B, C\right) & =y^{B+(C-B) v\left(u\left(\boldsymbol{y}^{\prime}\right)\right)} \\
v\left(u\left(\boldsymbol{y}^{\prime}\right)\right) & =A-\left(1-2 u\left(\boldsymbol{y}^{\prime}\right)\right)\left\lfloor\left\lfloor 0.5-u\left(\boldsymbol{y}^{\prime}\right)\right\rfloor+A \mid\right.
\end{aligned}
$$

where $\boldsymbol{y}^{\prime}$ is a secondary parameter vector, of domain [0,1] and $u$ is a reduction function. $A, B, C$, and $\boldsymbol{y}^{\prime}$ together determine the degree to which $y$ is biased by being raised to an associated power, values of $u\left(\boldsymbol{y}^{\prime}\right) \in[0,0.5]$ are mapped linearly onto $[B, B+(C-B) A]$, and values of $u\left(\boldsymbol{y}^{\prime}\right) \in[0.5,1]$ are mapped linearly onto $[B+(C-B) A, C]$.

\section{E.2.2 Shift}

Shift transformations move the location of optima. In the absence of any shift, all distance-related parameters would be extremal parameters, with optimal value at zero. Shift transformations can be used to set the location of parameter optima (subject to skewing by bias transformations), which is useful if medial and extremal parameters are to be avoided. It is recommended that all distance-related parameters be subjected to at least one shift transformation. The deceptive and multimodal shift transformations, defined below, make the corresponding problem deceptive and multimodal, respectively.

Linear $(A \in(0,1))$

$$
\text { s_linear }(y, A)=\frac{y-A}{\| A-y\rfloor+A \mid}
$$

$A$ is the value for which $y$ is mapped to zero.

Deceptive $(A \in(0,1), 0<B \ll 1,0<C \ll 1, A-B>0, A+B<1)$

$$
\begin{aligned}
\text { S_decept }(y, A, B, C)= & 1+(|y-A|-B)\left(\frac{\lfloor y-A+B\rfloor\left(1-C+\frac{A-B}{B}\right)}{A-B}\right. \\
& \left.+\frac{\lfloor A+B-y\rfloor\left(1-C+\frac{1-A-B}{B}\right)}{1-A-B}+\frac{1}{B}\right)
\end{aligned}
$$

$A$ is the value for which $y$ is mapped to zero, and is the global minimum of the transformation. $B$ is the "aperture" size of the well/basin leading to the global minimum at $A$, and $C$ is the value of the deceptive minima - there are always two deceptive minima.

multimodal $(A \in\{1,2, \cdots\}, B \geq 0,(4 A+2) \pi \geq 4 B, C \in(0,1))$

$$
\text { S_multi }(y, A, B, C)=\frac{1+\cos \left((4 A+2) \pi\left(0.5-\frac{|y-C|}{2(\lfloor C-y\rfloor+C)}\right)\right)+4 B\left(\frac{|y-C|}{2(\lfloor C-y\rfloor+C)}\right)^{2}}{B+2}
$$


$A$ controls the number of minima, $B$ controls the magnitude of the "hill sizes" of the multi-kodality, and $C$ is the value for which $y$ is mapped to zero. When $B=0,2 A+1$ values of $y$, one at $C$, are mapped to zero, and when $B \neq 0$, there are $2 A$ local minima, and one global minimum at $C$. Larger values of $A$ and smaller values of $B$ create more difficult problems.

\section{E.2.3 Reduction}

The reduction functions are

Weighted Sum $\left(|\boldsymbol{w}|=|\boldsymbol{y}|, w_{1} \cdots, w_{|y|}>0\right)$

$$
\mathrm{r} \_\operatorname{sum}(\boldsymbol{y}, \boldsymbol{w})=\frac{\sum_{i=1}^{|\boldsymbol{y}|} w_{i} y_{i}}{\sum_{i=1}^{|\boldsymbol{y}|} w_{i}}
$$

The constant weight vector $\boldsymbol{w}$ forces an algorithm to treat the parameter vector, $\boldsymbol{y}$, differently.

Non-separable $(A \in\{1, \cdots,|\boldsymbol{y}|\},|\boldsymbol{y}| \bmod A=0$

$$
\mathrm{r}_{-} \operatorname{nonsep}(\boldsymbol{y}, A)=\frac{\sum_{i=1}^{|\boldsymbol{y}|}\left(y_{i}+\sum_{j=0}^{A-2}\left|y_{i}+y_{1}+(i+j) \bmod \right| \boldsymbol{y} \mid\right)}{\frac{|\boldsymbol{y}|}{A}\left\lceil\frac{A}{2}\right\rceil\left(1+2 A-2\left\lceil\frac{A}{2}\right\rceil\right)}
$$

$A$ controls the degree of non-separability, noting that $\mathrm{r}_{-} \operatorname{non} \operatorname{sep}(\boldsymbol{y}, 1)=\mathrm{r} \_\operatorname{sum}(\boldsymbol{y}, 1)$

\section{E.3 Restrictions}

To ensure problems created using the WFG toolkit are well-designed, the following restrictions apply:

\section{Primary Parameters}

For any given transition vector, all the parameters of the originating transition vector must be employed exactly once as a primary parameter, counting parameters that appear independently as primary parameters, and in the same order in which the parameters appear in the originating transition vector.

\section{Secondary Parameters}

Care must be taken to avoid cyclical dependencies in b_param. If $a$ is a primary parameter of $\mathrm{b}$, and $b$ is a secondary parameter, then $a$ depends on $b$. If $b$ likewise depends on c, then $a$ depends, indirectly, on c. To prevent cyclical dependencies, no two parameters should be dependent on one another. A parameter should also not depend on itself. 


\section{Shifts}

Parameters should only be subjected to a maximum of one shift transformation.

\section{Reductions}

Reduction transformation should belong to transition vectors that are closer to the underlying parameter vector than any shift transformations.

b flat

When $A=0, \mathrm{~b}$ _flat should only belong to transition vectors that are further away from the underlying parameter vector than any shift or reduction transformation.

\section{Constants}

Constants must be fixed values and cannot be tied to the value of any parameter. The values and domains of the working parameters for the nine WFG test problems are defined as follows:

$$
\begin{aligned}
S_{k}=1: m & =2 k \\
A_{1} & =1 \\
A_{2: m-1} & = \begin{cases}0 \text { for WFG3 } \\
1 \text { otherwise }\end{cases}
\end{aligned}
$$

The settings for $S_{1: m}$ ensure that the POFs have dissimilar trade-off magnitudes, and the settings for $A_{1: m-1}$ ensure that the POFs are not degenerate, except in the case of WFG3, which has a one-dimensional POF.

\section{Domains}

The domains for the working parameters, defined below, are of dissimilar magnitude:

$$
z_{i=1: n, \max }=2 i
$$

\section{E.4 Test Problems}

The nine scalable test problems, summarised in Table E.1 are defined as follows: 


\section{WFG1}

$$
\begin{aligned}
\text { shape } \quad h_{k=1: m-1} & =\text { convex }_{j} \\
h_{m} & =\operatorname{mixed}_{j}, \text { with } \alpha=1 \text { and } A=5 \\
\mathrm{t}^{1} \quad t_{i=1: j}^{1} & =y_{i} \\
t_{i=j+1: n}^{1} & =\mathrm{s}_{-} \operatorname{linear}\left(y_{i}, 0.35\right) \\
\mathrm{t}^{2} \quad t_{i=1: j}^{2} & =y_{i} \\
t_{i=j+1: n}^{2} & =\mathrm{b}_{-} \text {flat }\left(y_{i}, 0.8,0.75,0.85\right) \\
t_{i=1: n}^{3} & =\mathrm{b}_{-} \operatorname{poly}\left(y_{i}, 0.02\right) \\
\mathrm{t}^{3} \quad t_{i=1: m-1}^{4} & =\mathrm{r}_{-} \operatorname{sum}\left(\left(y_{(i-1) j /(m-1)+1}, \cdots, y_{i j /(j-1)}\right),\right. \\
& (2((i-) j /(m-1)+1), \cdots, 2 i j /(m-1))) \\
t_{k}^{4} & =\mathrm{r}_{-} \operatorname{sum}\left(\left(y_{j+1}, \cdots, y_{n}\right),(2 k(j+1), \cdots, 2 n)\right)
\end{aligned}
$$

\section{WFG2}

shape $\quad h_{k=1: m-1}=$ convex $_{j}$

$$
\begin{aligned}
h_{m} & =\operatorname{disc}_{j}, \text { with } \alpha=\beta=1 \text { and } A=5 \\
\mathrm{t}^{1} \quad t_{i=1: j}^{1} & =y_{i} \\
t_{i=j+1: n}^{1} & =\mathrm{s} \_\operatorname{linear}\left(y_{i}, 0.35\right) \\
\mathrm{t}^{2} \quad t_{i=1: j}^{2} & =y_{i} \\
t_{i=j+1: n}^{2} & =\mathrm{r}_{-} \operatorname{nonsep}\left(\left(y_{j+2(i-j)-1}, y_{j+2(i-j)}\right), 2\right) \\
\mathrm{t}^{3} \quad t_{1: m-1}^{3} & =\mathrm{r}_{-} \operatorname{sum}\left(\left(y_{(i-1) j /(m-1)+1}, \cdots, y_{i j /(j-1)}\right),(1, \cdots, 1)\right) \\
t_{m}^{3} & =\mathrm{r}_{-} \operatorname{sum}\left(\left(y_{j+1}, \cdots, y_{j+l / 2},(1, \cdots, 1)\right)\right.
\end{aligned}
$$

\section{WFG3}

shape $\quad h_{k=1: m}=$ linea $_{j}$

$$
\begin{aligned}
\mathrm{t}^{1} \quad t_{i=1: j}^{1} & =y_{i} \\
t_{i=j+1: n}^{1} & =\mathrm{s}_{-} \operatorname{linear}\left(y_{i}, 0.35\right) \\
\mathrm{t}^{2} \quad t_{i=1: j}^{2} & =y_{i} \\
t_{i=j+1: n}^{2} & =\mathrm{r}_{-} \operatorname{nonsep}\left(\left(y_{j+2(i-j)-1}, y_{j+2(i-j)}\right), 2\right) \\
\mathrm{t}^{3} \quad t_{1: m-1}^{3} & =\mathrm{r}_{-} \operatorname{sum}\left(\left(y_{(i-1) j /(m-1)+1}, \cdots, y_{i j /(j-1)}\right),(1, \cdots, 1)\right) \\
t_{m}^{3} & =\mathrm{r}_{-} \operatorname{sum}\left(\left(y_{j+1}, \cdots, y_{j+l / 2},(1, \cdots, 1)\right)\right.
\end{aligned}
$$

\section{WFG4}

shape $\quad h_{k=1: m}=$ concave $_{j}$

$$
\begin{aligned}
\mathrm{t}^{1} \quad t_{i=1: n}^{1} & =\mathrm{s} \_\operatorname{multi}\left(y_{i}, 30,10,0.35\right) \\
\mathrm{t}^{2} \quad t_{i=1: m-1}^{2} & =\mathrm{r}_{-} \operatorname{sum}\left(\left(y_{(i-1) j /(m-1)+1}, \cdots, y_{i j /(j-1)}\right),(1, \cdots, 1)\right) \\
t_{m}^{2} & =\mathrm{r} \_\operatorname{sum}\left(\left(y_{j+1}, \cdots, y_{n},(1, \cdots, 1)\right)\right.
\end{aligned}
$$




\section{WFG5}

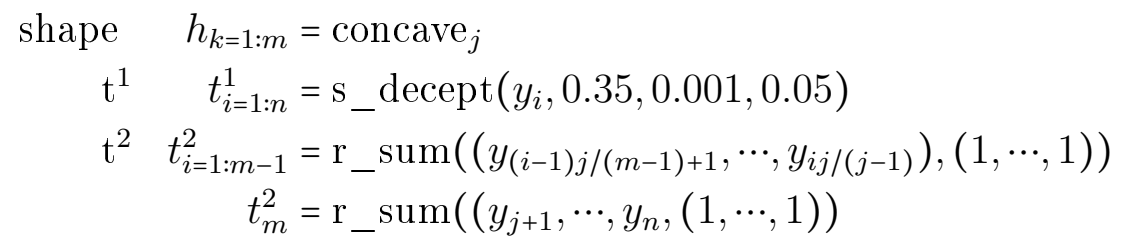

\section{WFG6}

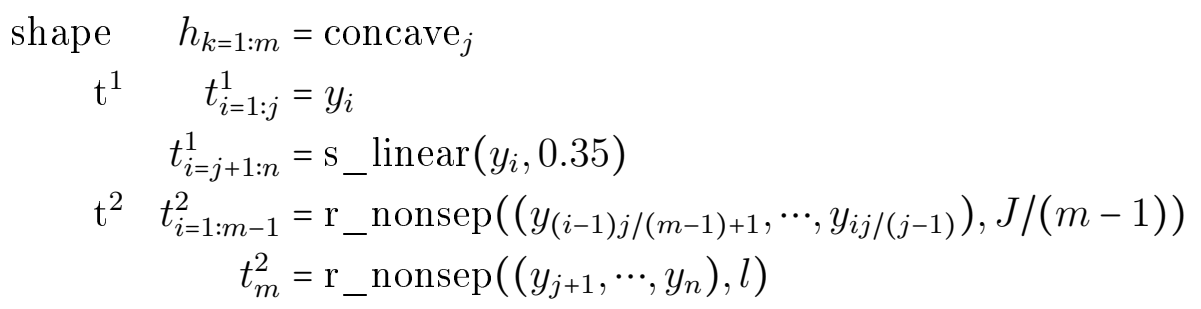

\section{WFG7}

shape $\quad h_{k=1: m}=$ concave $_{j}$

$$
\begin{aligned}
\mathrm{t}^{1} \quad t_{i=1: j}^{1} & =\mathrm{b}_{-} \operatorname{param}\left(y_{i}, \mathrm{r} \_\operatorname{sum}\left(\left(y_{i+1}, \cdots, y_{n}\right),(1, \cdots, 1)\right), \frac{0.98}{49.98}, 0.02,50\right) \\
t_{i=j+1: n}^{1} & =y_{i} \\
\mathrm{t}^{2} \quad t_{i=1: j}^{2} & =y_{i} \\
t_{i=j+1: n}^{2} & =\mathrm{s} \_\operatorname{linear}\left(y_{i}, 0.35\right) \\
\mathrm{t}^{3} \quad t_{i=1: m-1}^{3} & =\mathrm{r}_{-} \operatorname{sum}\left(\left(y_{(i-1) j /(m-1)+1}, \cdots, y_{i j /(j-1)}\right),(1, \cdots, 1)\right) \\
t^{3}{ }_{m} & =\mathrm{r}_{-} \operatorname{sum}\left(\left(y_{j+1}, \cdots, y_{n},(1, \cdots, 1)\right)\right.
\end{aligned}
$$

\section{WFG8}

$$
\begin{aligned}
\text { shape } \quad h_{k=1: m} & =\text { concave }_{j} \\
\mathrm{t}^{1} \quad t_{i=1: j}^{1} & =y_{i} \\
t_{i=j+1: n}^{1} & =\mathrm{b} \_\operatorname{param}\left(y_{i}, \mathrm{r} \_\operatorname{sum}\left(\left(y_{i+1}, \cdots, y_{n}\right),(1, \cdots, 1)\right), \frac{0.98}{49.98}, 0.02,50\right) \\
t^{2} \quad t_{i=1: j}^{2} & =y_{i} \\
t_{i=j+1: n}^{2} & =\mathrm{s}_{-} \operatorname{linear}\left(y_{i}, 0.35\right) \\
\mathrm{t}^{3} \quad t_{i=1: m-1}^{3} & =\mathrm{r}_{-} \operatorname{sum}\left(\left(y_{(i-1) j /(m-1)+1}, \cdots, y_{i j /(j-1)}\right),(1, \cdots, 1)\right) \\
t^{3}{ }_{m} & =\mathrm{r}_{-} \operatorname{sum}\left(\left(y_{j+1}, \cdots, y_{n},(1, \cdots, 1)\right)\right.
\end{aligned}
$$




\section{WFG9}
shape $\quad h_{k=1: m}=$ concave $_{j}$
$\mathrm{t}^{1} \quad t_{1: n-1}^{1}=\mathrm{b} \_$param $\left(y_{i}, \mathrm{r} \_\operatorname{sum}\left(\left(y_{i+1}, \cdots, y_{n}\right),(1, \cdots, 1)\right), \frac{0.98}{49.98}, 0.02,50\right)$

$$
t_{n}^{1}=y_{n}
$$$$
\mathrm{t}^{2} \quad t_{i=1: j}^{2}=\mathrm{s} \_\operatorname{decep}\left(y_{i}, 0.35,0.001,0.05\right)
$$$$
t_{i=j+1: n}^{2}=\mathrm{s} \_ \text {multi }\left(y_{i}, 30,95,0.35\right)
$$$$
\mathrm{t}^{2} \quad t_{i=1: m-1}^{2}=\mathrm{r} \_ \text {nonsep }\left(\left(y_{(i-1) j /(m-1)+1}, \cdots, y_{i j /(j-1)}\right), J /(m-1)\right)
$$$$
t_{m}^{2}=r_{-} \text {nonsep }\left(\left(y_{j+1}, \cdots, y_{n}, l\right)\right.
$$

Table E.1: Properties of the WFG problems

\begin{tabular}{llll}
\hline Name & Separability & modality & Shape \\
\hline WFG1 & Separable & Unimodal & Convex, mixed \\
WFG2 & Non-separable & Unimodal/multimodal & Convex, disconnected \\
WFG3 & Non-separable & Unimodal & Linear, degenerate \\
WFG4 & Separable & Multimodal & Concave \\
WFG5 & Separable & Multimodal & Concave \\
WFG6 & Non-separable & Unimodal & Concave \\
WFG7 & Separable & Unimodal & Concave \\
WFG8 & Non-separable & Unimodal & Concave \\
WFG9 & Non-separable & Multimodal, deceptive & Concave \\
\hline
\end{tabular}

